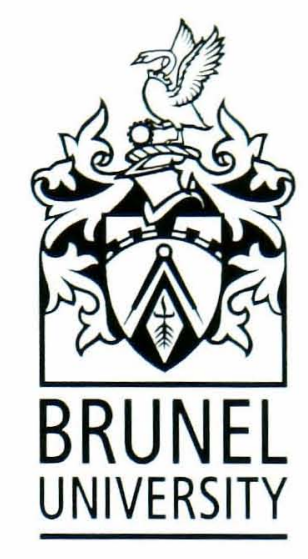

\title{
A NEW METHOD FOR POWER QUALITY IMPROVEMENT
}

A thesis submitted for the degree of Doctor of Philosophy

by

\section{Ismail Kasikci}

Department of Electronic and Computer Engineering

Brunel University

March 2000 


\section{Acknowledgements}

This research work was performed at the Technical University of Darmstadt and at Brunel University, London.

I would like to express my deep sense of gratitude to Dr. M. Darwish for his invaluable guidance, constant encouragement and enlightening discussions during the course of this research and preparation of the thesis.

I am indebted to Dr. El-Habrouk for his technical assistance, help and comments.

I am also grateful to the Head of the Department of Electronic \& Computer Engineering at Brunel, for being allowed to perform this research work.

I would also especially like to thank Prof. Dr. Strack and Dr. Steck at the Technical University of Darmstadt for their proof reading and suggestions.

It was a pleasure for me to work at the Technical University of Darmstadt and to use the computer facility there.

Finally, I am grateful to my wife for her unfailing moral support and patience throughout the period of this research. 


\begin{abstract}
A new control method for active power filters in conjunction with a passive filter circuit are presented and analysed in this thesis. A new technique for load modelling is introduced in order to enable the design of compensators to improve the power factor and to reduce harmonic levels in electrical power systems. The principles of analysis, design, operation and control of the new circuit equipped with IGBTs are presented. This enables the compensation of rapidly changing loads and reactive power.
\end{abstract}

A special circuit equipped with IGBTs is able to compensate for the reactive power and harmonic currents of different orders. The important aspect of the present work is based on the compensator control circuit for power factor correction and harmonic elimination, and its application. This new configuration improves the rating of the active power filter, reducing power losses in the switches compared to existing and newly developed active filters. Furthermore, it is very stable in operation and much faster by a factor of 20 . The thesis also presents a detailed mathematical modelling of the proposed system with frequency and time domain equations. The frequency response of the proposed system is also discussed.

This new proposal has been checked using a dedicated software simulation program, which was specifically developed for this purpose. An experimental set-up has been designed and implemented in order to apply the new method using IGBTs as well as some other devices.

This thesis also presents a critical literature survey, which provides a critical overview of previous work relevant to the power quality improvement, reactive power compensation and active filtering. 


\section{List of Symbols}

$B_{A C, s} \quad$ Amplitude of the induction of the AC-content at the switching frequency

C Capacitance [F]

$C_{E} \quad$ Ground-Line capacitance $[\mathrm{F}]$

$C_{F} \quad$ Filter capacitance $[\mathrm{F}]$

$C_{l d} \quad$ Load capacitance $[\mathrm{F}]$

$C_{D C} \quad$ Inverter DC capacitor [F]

$C_{L V} \quad$ Low voltage capacitor [F]

$C_{M V} \quad$ Medium voltage capacitor $[\mathrm{F}]$

$e_{B} \quad$ Material-dependent constants of the core

$e_{f} \quad$ Material-dependent constants of the core

$E_{I G B T}$ Energy dissipation of the IGBT [Ws]

$f \quad$ Frequency [Hz]

$f_{h}(s) \quad$ Laplace transform of harmonic calculation function $[\mathrm{Hz}]$

$f_{l} \quad$ Fundamental frequency at supply voltage [Hz]

$f_{r} \quad$ Resonance frequency $[\mathrm{Hz}]$

$f_{S} \quad$ Switching frequency $=$ Sampling frequency $[\mathrm{Hz}]$

$G_{C} \quad$ Controller transfer function

$G_{I} \quad$ Inverter transfer function

$\underline{G}(s) \quad$ Transfer matrix

I Current [A]

$i_{C} \quad$ Capacitor current [V]

$i_{\text {Col }}$ Collector current [V]

$I_{C E} \quad$ Ground-Earth capacitor current [V]

$I_{C N} \quad$ Nominal value of the capacitor current [V]

$i_{\text {Comp }}$ Compensation current [A]

$i_{h} \quad$ Harmonic current [A]

$I_{M}^{\text {Rated }}$ Rated motor current

$I_{i n,}, \quad$ rms-Inverter current [A]

$i_{i n v} \quad$ Inverter current $[\mathrm{A}]$

$i_{l d} \quad$ Load current [A]

$i_{\text {Reactive }}$ Reactive current [A]

$\hat{i}_{i n v}^{*} \quad$ Peak value of Inverter reference current [A]

$I_{s c} \quad$ Initial short circuit current [A]

$I_{F} \quad$ Filter current [A]

$I_{S} \quad$ rms-Supply current [A]

$\underline{I}_{h} \quad$ Harmonic current [A]

$I_{h_{i}}$ Harmonic current [A]

$I_{l, Y} \quad$ Low voltage current [A]

$i_{\text {S. }} \quad$ Instantaneous input supply current [A]

$I_{\text {Starting }}$ Starting current [A] 
$\hat{i}_{S} \quad$ Peak value of supply current [A]

$I_{\delta} \quad$ Control band width $\quad[\mathrm{A}]$

$I_{\text {rem }} \quad$ Earth remaining current [A]

$K_{1} \quad$ Inverter gain

$L \quad$ Inductance $[\mathrm{H}]$

$L_{\text {Comp }}$ Compensation inductance $[\mathrm{H}]$

$L_{\text {inv }} \quad$ Inverter inductance $[\mathrm{H}]$

$L_{F} \quad$ Filter inductance $[\mathrm{H}]$

$L_{S} \quad$ Supply inductance $[\mathrm{H}]$

$L_{T l} \quad$ Fundamental frequency inductance of the transformer [H]

$k \quad$ Time step index

$m_{L C L} \quad$ Current correction factor of the desired value

$N \quad$ Transformer ratio

$p \quad$ Reactor/capacitor ratio $[\%]$

$P \quad$ Active Power [kW]

$p_{B} \quad$ Material-dependent constants of the core

$P_{C} \quad$ Conduction losses [W]

$P_{\text {core }}$ Core losses [W]

$P_{C l} \quad$ Copper losses $[\mathrm{W}]$

$P_{l d} \quad$ Load active power $[\mathrm{KW}]$

$Q \quad$ Reactive Power (inductive positive) [KVAR]

$Q F \quad$ Circuit quality

$Q_{C} \quad$ Capacitor power [KVAR]

$Q_{F} \quad$ Fundamental reactive power [KVAR]

$R \quad$ Resistance $[\Omega]$

$R_{F} \quad$ Filter resistance $[\Omega]$

$r_{C o m p}$ Internal resistance of the compensator inductance $[\Omega]$

$r_{f} \quad$ slope resistance $[\Omega]$

$r_{i n v} \quad$ Internal resistance of the inverter inductance [ $\Omega$ ]

$r_{S} \quad$ Internal resistance of the supply reactance [ $\Omega$ ]

$R_{E} \quad$ Earth resistance [ $\Omega$ ]

$R_{l d} \quad$ Load resistance $[\Omega]$

$R_{T} \quad$ Transformer resistance [ $\Omega$ ]

$R_{T h} \quad$ Harmonic transformer resistance [ $\left.\Omega\right]$

$S_{\text {Comnected }}^{\text {Rated }}$ Rated apparent power at the PCC [KVA]

$S_{C^{\prime}} \quad$ Connecting power [KVA]

$S_{S P} \quad$ Single phase apparent power [KVA]

$P_{S} \quad$ Supply power $[\mathrm{kW}]$

$S_{M}^{\text {Rated }}$ Rated apparent motor power [KVA]

$S_{s c}^{\infty} \quad$ Subtransient short circuit level for infinite busbar [MVA]

$S_{s c M}$ Medium voltage subtransient short circuit level for a certain busbar [MVA]

$S_{s c L l}$ Low voltage subtransient short circuit level for a certain busbar [MVA]

$S_{T}^{\text {Rated }}$ Rated transformer apperant power [KVA]

$\sin \varphi \quad$ Reactive power factor

$t \quad$ Time [s] 
$t_{l} \quad$ Current decay in time 1 [s]

$t_{d} \quad$ Delay time [s]

$t_{f} \quad$ Fall time [s]

$t_{r i} \quad$ Rise time [s]

$t_{\text {tail }}$ Tail time [s]

$T \quad$ Duration period $[1 / \mathrm{s}]$

$T_{D} \quad$ Switching delay time [s]

$T_{S} \quad$ Sampling time (switching time) [s]

$V_{C} \quad$ Capacitor voltage [V]

$V_{C E} \quad$ Collector-Emitter voltage [V]

$V_{D C} \quad$ Inverter DC voltage [V]

$V_{E} \quad$ Magnetic volume [V]

$V_{F} \quad$ Conduction state of voltage [V]

$v_{l d} \quad$ Load supply voltage [V]

$v_{S} \quad$ Supply voltage [V]

$V_{S} \quad$ rms-Supply voltage [V]

$V_{G}^{\text {Rated }}$ Nominal voltage of the generator [V]

$V_{M}^{\text {Rated }}$ Rated voltage of the motor [V]

$v_{\text {Stray }}^{\text {Rated }} \quad \%$ Rated voltage losses of the transformer

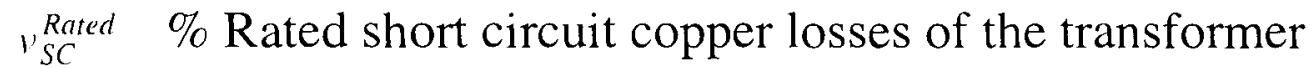

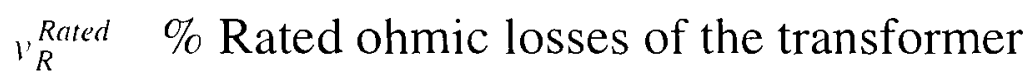

$\hat{v}_{S} \quad$ Peak value of supply voltage [V]

$\underline{V}_{h} \quad$ Harmonic voltage [V]

$V_{h_{i}} \quad i^{\text {th }}$ Harmonic voltage [V]

$X \quad$ Reactance $[\Omega]$

$X_{i m}, \quad$ Inverter reactance $[\Omega]$

$X_{S} \quad$ Network reactance $[\Omega]$

$X_{T h} \quad$ Harmonic transformer reactance [S]

$\underline{X}(s) \quad$ Input matrix

$Y \quad$ Admittance [S]

$\underline{Y}(s) \quad$ Output matrix

$Z \quad$ Impedance $[\Omega]$

$Z_{C} \quad$ Compensation impedance $[\Omega]$

$Z_{l d} \quad$ Load impedance $[\Omega]$

$Z_{M}^{\text {Rated }}$ Rated motor impedance $[\Omega]$

$Z_{T}^{\text {Rated }}$ Rated transformer impedance $[\Omega]$

$Z_{F} \quad$ Filter impedance $[\Omega]$

$\underline{Z}_{h} \quad$ Filter harmonic impedance [ $\left.\Omega\right]$

$Z_{F_{i}} \quad i^{t h}$ Filter impedance [ $\Omega$ ]

$Z_{h_{i}} \quad i^{t h}$ harmonic impedance $[\Omega]$

$Z_{s,} \quad i^{t / h}$ Supply impedance $[\Omega]$

$Z_{S} \quad$ Supply impedance $[\Omega]$

$\omega$ Radian frequency $[\mathrm{rad} / \mathrm{s}]$ 
$\omega_{l} \quad$ Supply radian frequency $[\mathrm{rad} / \mathrm{s}]$

$\varphi_{\text {Id }} \quad$ Load angle degree

$\cos \varphi$ Power factor

$\varphi_{M}^{\text {Rated }}$ Rated motor power factor angle 


\section{Greek Symbols}

$\begin{array}{ll}\alpha & \text { Control angle } \\ \varphi_{n} & \text { Desired angle of the phase correction } \\ \varphi & \text { Phase angle of the inverter } \\ v & \text { Ordinal number } \\ \omega & \text { Radian frequency } \\ \vartheta & \text { Temperature }\end{array}$

\section{Indices}

$i \quad i^{\text {th }}$-Harmonics

$n \quad$ Ordinal number

$h \quad$ Harmonics

\section{Abbreviations}

$A F \quad$ Active Filter

$A P F \quad$ Active power filter

AI Artificial intelligence

$C E \quad$ Collector-Emitter

$D \quad$ Diode

$D C \quad$ Direct current

DCOM Dynamic compensation

EN Europa Norm

FS Filter System

$F \quad$ Filter

$G \quad$ Generator

$H V \quad$ High Voltage

IEC International Electrotechnical Commission

$I G B T$ Insulated Gate Bipolar Transistor

$L V \quad$ Low Voltage

$L \quad$ Load

$N L L \quad$ Non-linear load

$M V \quad$ Medium Voltage

$M V C$ Capacitive reactive power of the medium voltage

$M \quad$ Motor

$P F \quad$ Power Factor

$P C C$ Point of Common Coupling

$P F C$ Power Factor Correction

$R P C$ Reactive Power Compensation

$S C$ Series Compensation

SVC Static VAR Compensation

$T$ Transformer

TCR Thyristor controlled reactor

TSC Thyristor switched capacitor 
THD Total harmonic distortion

VAR Volt-Amp-Reactor

$V D E$ Association of German Electrical Engineers

VSI Voltage source inverter 


\section{Table of Contents}

Acknowledgements

Abstract

List of Symbols

List of Greek Symbols, Indices and Abbreviations

Table of Contents

\section{Chapter 1 Introduction}

1.1 Problem Overview

1.2 Layout of this Thesis

\section{Chapter 2 Literature Review and Previous Compensation Methods}

2.1 Introduction

$2.2 \quad$ Fundamentals of Reactive Power 8

2.3 Network Components Characteristics 9

2.4 Relationship between Voltage and Reactive Power 10

$\begin{array}{lll}2.5 & \text { Reactions on the Mains } & 12\end{array}$

2.5.1 Fundamental Concepts $\quad 12$

$\begin{array}{lll}\text { 2.5.2 Voltage Asymmetries } & 15\end{array}$

2.5.3 Voltage Fluctuations 15

2.5.4 Harmonic Oscillations $\quad 17$

2.5.5 Voltage Drops in the Networks $\quad 17$

$\begin{array}{lll}2.5 .6 & \text { Voltage Distortions } & 17\end{array}$

$\begin{array}{lll}\text { 2.6 Reactive Power Compensation in Transmission Systems } & 17\end{array}$ 
2.6.1 Steady-State Voltage Control

2.6.2 Reduction of Temporary Over-voltages

2.6.3 Synchronous Stability Improvements

2.6.4 Prevention of Voltage Collapse

2.6.5 Extensive Cable Network

2.7 Reactive Power Compensation in Distribution Systems 25

2.8 High-Power Industrial Systems

2.8.1 Supplying Steady-State Reactive Power and Voltage Control

2.8.2 Reduction of Voltage Fluctuation 28

2.8.3 Reduction of Voltage Drop during Large Motor Starts

2.9 Reactive Power Compensation Devices 30

2.9.1 Shunt Reactors 30

2.9.2 Shunt Capacitors 31

2.9.3 Synchronous Condenser

2.9.4 Thyristor-Controlled Static Compensators

2.9.4.1 Thyristor-Switched Capacitor Compensators 34

2.9.4.2 Thyristor-Controlled Reactor Compensators 35

2.10 Series capacitors 36

2.11 Passive filter circuits 37

2.12 Shunt Active Power Filters

2.12.1 Standard Active Filter Circuits

2.12.2 Switched Capacitor Active Filter Circuits

2.12.3 Lattice Structured Active Filter Circuits

2.12.4 Voltage Controller Active Filter Circuit

2.13 Series Active Filter 
2.14 Reactive Power Optimisation in Power Systems

2.15 Expert Systems, Fuzzy Logic, and Neural Network Application in Power Systems 45

2.16 Summary

\section{Chapter 3 A New Method for Power Quality Improvement}

$\begin{array}{lll}3.1 & \text { Introduction } & 48\end{array}$

3.2 Proposed Circuit and its Control Strategy 49

3.3 Operation of the Proposed Circuit 62

3.4 Exact Modelling of the Proposed Circuit 64

3.4.1 Frequency-Domain Modelling $\quad 65$

$\begin{array}{lll}\text { 3.4.2 State-Space Modelling } & 75\end{array}$

3.5 Effect of Filter Connection to the Power System 76

3.6 Numerical Example $\quad 81$

3.6.1 Connection of a Motor Drive Load to the Low Voltage Power System 81

3.6.2 Compensator Parameter Determination 83

$\begin{array}{lll}3.7 & \text { Summary } & 87\end{array}$

Chapter 4 Analytical Load Models for Power System Components

$\begin{array}{lll}\text { 4.1 Introduction } & 88\end{array}$

4.2 Modelling of the Power System $\quad 89$

4.3 Analysis of the Characteristics of the Power Systems 91

4.3.1 Transformer Impedance 91

4.3.2 Ohmic Loads 92

4.3.3 Ohmic and Inductive Load (Motor)

4.3.4 Ohmic, inductive and Capacitive Load (Capacitor) of 
4.3.5 Medium Voltage Power System 95

4.3.6 Losses in the Power System 96

4.3.7 Earth Leakage $\quad 96$

4.4 Proposed Load Model for Power System Components 97

4.5 Simulation Results of the Proposed Model 97

4.6 Discussion of the Proposed Model 101

$\begin{array}{lll}4.7 & \text { Summary } & 102\end{array}$

\section{Chapter 5 Simulation Results}

5.1 Introduction $\quad 103$

5.2 Losses in the Proposed System 103

5.2.1 Device Losses 104

5.2.2 Filter Losses 105

5.3 Simulation of the proposed Inverter Power Circuit 106

5.3.1 Simulation of the Losses 106

5.3.2 PWM and Hysteresis Switching Techniques 108

5.3.3 Effect of the Passive Filter 114

5.3.4 Harmonic Measurements in a real $20 \mathrm{kV}$ and $400 \mathrm{~V}$ Power 115

5.3.5 Simulation Results for an inductive Load in the Power System 118

5.3.6 Simulation Results for given Non-linear Load Output $\quad 119$

5.4 Summary $\quad 120$

Chapter 6 Experimental Set-up and Practical Results

6.1 Introduction

6.2 Choice of Switching Devices 
6.3 Inverter and AC-Filter Design $\quad 122$

$\begin{array}{llr}6.4 & \text { Experimental Set-up } & 124\end{array}$

$\begin{array}{llr}\text { 6.5 Practical Results } & 129\end{array}$

6.5.1 EMC Compatibility and Choice of PWM and Hysteresis Control 129

6.5.2 Choice of Inverter Inductance $\quad 132$

6.5.2.1 Non-linear Load with $L_{i n v}=4 \mathrm{mH}$

6.5.2.2 Non-linear Load with $L_{i n v}=2 \mathrm{mH}$

6.5.2.3 Non-linear Load with $L_{i n \nu}=1 \mathrm{mH}$

6.5.3 Power Factor Correction Case $\left(L_{i n v}=1 \mathrm{mH}\right) \quad 135$

$\begin{array}{lll}6.6 & \text { Analysis of the Losses } & 140\end{array}$

6.7 Time Response 141

6.8 Summary 142

\section{Chapter 7 Conclusions and Future Work}

7.1 General Conclusions 143

7.2 Discussion of Results 145

$\begin{array}{lll}7.3 & \text { Practical Applications } & 149\end{array}$

$\begin{array}{ll}7.4 & \text { Suggestions for Future Work } \\ \end{array}$

List of References

\section{Appendices}

A. Control Block Diagrams of the Transfer Functions of the Filter A.1

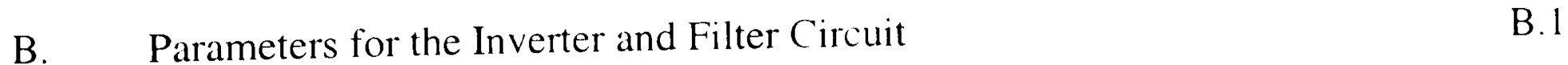

C. Software Program for the Evaluation of the Proposed Control System C.I 
$\begin{array}{ll}\text { D. Circuit Implementation } & \text { D.1 }\end{array}$

E. Power and Control Circuit E.1

F. Overview and Construction of the Proposed System $\quad$ F.1

G. List of Publications G.1 


\section{Chapter 1}

\section{Introduction}

\subsection{Problem Overview}

Power factor improvement, reactive power compensation and harmonics in electrical power systems with non-sinusoidal supply have been subjects of concern for a long time [1-5]. As a consequence, various types of compensators have been developed to improve the efficiency of power transmission and to increase the power factor [2-9].

On the load side, the number of power and commercial electronic circuits are increasing. Because of this, the harmonics are also increasing on the low and medium voltage power system.

To meet regulations of IEC 1000-2-2 and IEEE Std. 519 [10,11], power systems must be free of harmonics and electrical pollution. This dictates the use of special equipment to overcome these problems.

The task of the electrical power system is transmission of energy economically and with low loss from the generation to the end-user. The whole power supply system (mains. cables and other equipment) should be designed for optimum efficiency. The performance of the loads can not be guaranteed if the supply voltage is not sinusoidal. unsymmetrical or deviates from the rated value. 
As matter of fact, the power network is polluted, carrying non-linear currents and voltages. The result is that the frequency dependent impedance has a distorted supply and the load then operates as a noise source [12]. The effects of disturbances on the mains occur due to the absorption and/or generation of reactive power, harmonic currents and asymmetrical loads [13].

The network impedance shows high inductive properties. Hence, the capacitors and filters should be connected in shunt at different points in the power supply network in order to get the same values of impedance at every node [14].

Changes in the reactive power in the network cause voltage fluctuations which, in turn, lead to failures and flicker. Controllable-compensation [3] limits these over-voltages but themselves cause harmonics, high power losses and excessive noise levels.

This thesis presents a new controller system equipped with IGBTs for power factor improvement as well as harmonic compensation. The operating principles and applications of the proposed system are discussed and a new harmonic compensation method, generating optimal switching control algorithms and patterns using real situations in power networks, is presented. 


\subsection{Layout of this Thesis}

The thesis is organised as follows:

Chapter 1 briefly introduces the power quality problems in three-phase systems and their solutions.

Chapter 2 presents an overall literature review and explains most of the common compensation methods and disturbances in electrical systems. Furthermore, it presents various reactions of the power quality problem on the power systems.

Chapter 3 describes a new control method with IGBTs for power factor improvement, its principle of operation, its application and a new harmonic compensation method in power systems generating optimal switching patterns using real power system data and waveforms. The control systems are explained and described.

As an example, design steps are introduced to show the procedure for evaluating the filter parameters for specific requirements.

Chapter 4 deals with the analytical load modelling for reactive power compensation. It introduces a software program for computing the network impedance and harmonics and calculates the compensation and filter circuit characteristics of the new method for analysis purposes of the power system.

Chapter 5 presents the simulation results of the implementation of the proposed controller with data extracted from real power system situations. The simulation 
includes the analysis of several aspects of the proposed controller and control and switching algorithms, which are explained during the course of the chapter.

The practical implementation of the proposed system is performed in a laboratory prototype. The description of the implementation process is presented in chapter 6 , in conjunction with the practical results.

Chapter 7 concludes the thesis with the discussion of the results obtained from the investigation and their applications. Suggestions for future work are also included. 


\section{Chapter 2}

\section{Literature Review and Previous Compensation Methods}

\subsection{Introduction}

In the past, many publications attempted to solve the problem of reactive power compensation and harmonics in electric power systems and there is a requirement for this work to be continued [1-3]. The previous work can be summarised as:

1. Practical definitions for power with non-sinusoidal waveforms and unbalanced loads

2. Real power loss minimisation

3. Improvements in voltage profiles

4. Optimal reactive power control

5. Estimation of harmonic components

6. Reduction of harmonic distortion in the networks

The following is a detailed review of the published papers and the literature on this subject.

Reactive power appears in every AC power system. Many loads consume not only active but also reactive power. The electric grid itself both consumes and produces reactive power. Transmission and distribution of electric power involves reactive power losses due to the series inductance of transformers, overhead lines and underground cables. Lines and cables also generate reactive power due to their shunt capacitance: this generation of reactive power is. however, only of significance at 
high system voltages. During the steady-state operation of an AC power system. the active power production must match the consumption plus the losses, otherwise the frequency will change [10]. There is an equally strong coupling between the reactive power balance of a power system and the voltages. In itself, a reactive power balance will always inherently be present, but with unacceptable voltages if the balance is not a proper one. The reactive power balance of an electrical power system also influences the active losses of the network, the heating of components and, in some cases, the stability of the system.

In contrast to the active power balance, which has to be effected by means of the generators only, a proper reactive power balance can, and often must be, effected both by the generators and by dispersed special reactive devices, producing or absorbing reactive power. The use of shunt reactive devices, i.e. shunt compensation, is a straightforward reactive power compensation method. The use of series capacitors, i.e. series compensation, is a line reactance compensation method.

No special reactive compensation devices were used in the early AC power systems, because the generators were located close to the loads. As networks became more extensive, synchronous motors, small synchronous condensers and static shunt capacitors were adopted for power-factor correction. Larger synchronous condensers were installed in transmission systems. Along with the development of more efficient and economic capacitors, there has been a phenomenal growth in the use of shunt capacitors as a means of furnishing reactive power, particularly within distribution systems. With the introduction of extra-high voltage lines, shunt reactors and series capacitors became important compensation devices [3]. The latest development is the 
thyristor-controlled static compensator, which is now a well-established device not only in high-power industrial networks but also in transmission systems. Fig. 2.1 shows how different compensation devices are often installed in power systems.

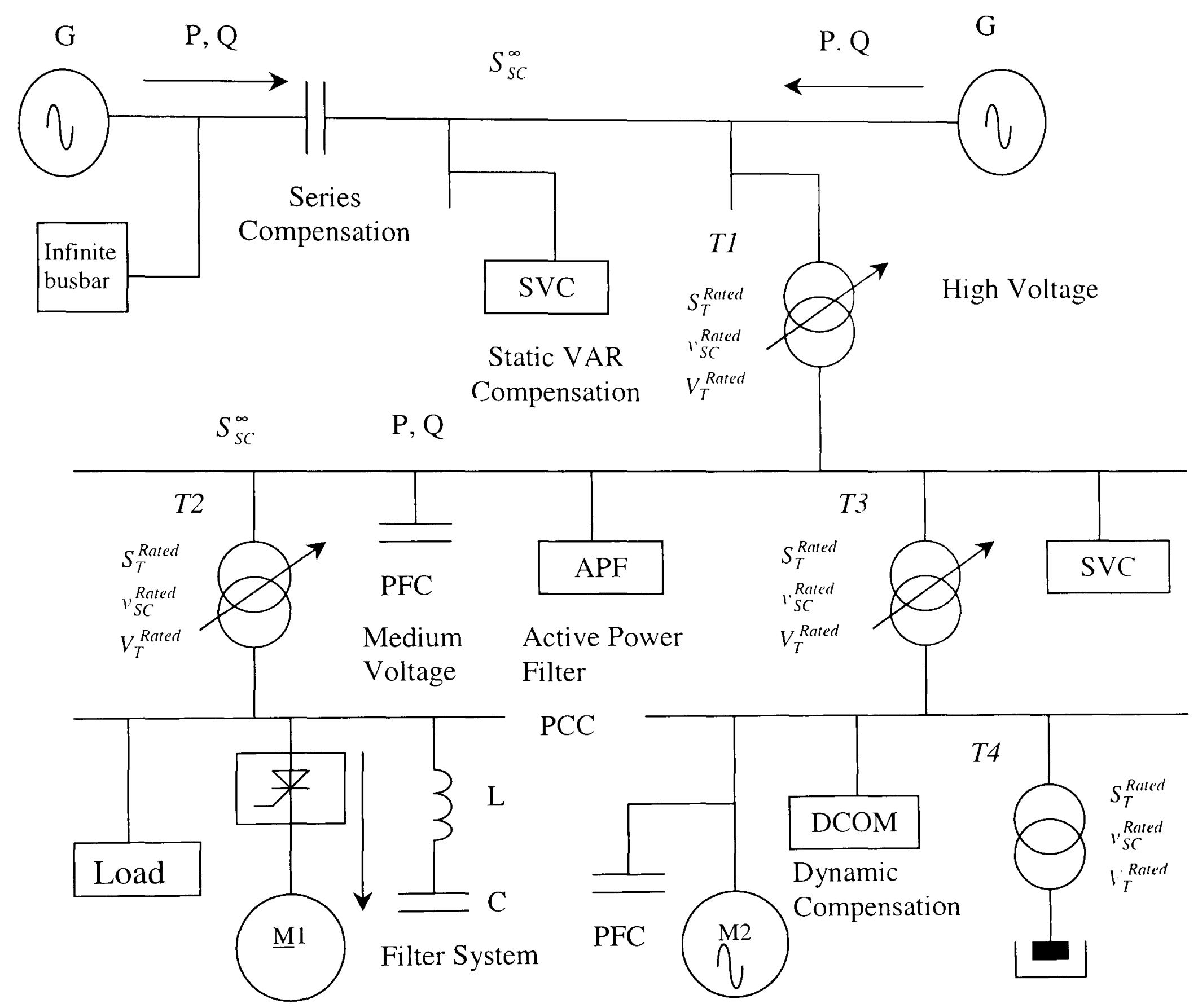

Low Voltage

Fig.2.1: Different Compensation Devices in a typical Transmission and

Distribution Systems

Relatively speaking, transmission systems form those parts of power systems conveying large amounts of electrical power. They link the generating sources with 
distribution systems and interconnect various parts of the power system. Distribution systems form the continuous links to the consumer.

\subsection{Fundamentals of reactive power}

With phasor description of voltage and current, the reactive power supplied to an $\mathrm{AC}$ circuit is the product of the voltage and the reactive component of the current, this reactive current component being in quadrate with the voltage. Using the IEC sign convention, reactive power is said to be produced or generated by over-excited synchronous machines and capacitors, and consumed or absorbed by under-excited synchronous machines and inductors.

Reactive power can be considered as a convenient calculation quantity, giving information about the watt-less current, which greatly influences voltages and active losses [15-16].

Individual loads may, of course, vary over shorter or longer time spans. The composites loads on a power system (each one being the total load of a certain area), usually vary with the time of the day, the day of the week and the season of the year, and may also grow from year to year. The consumer demand for reactive power varies in a somewhat similar way to the demand for active power.

The resultant active power demand of a power system varies as the variation of the total load. The resultant reactive power demand may vary considerably more due to the changing series reactive power losses in the networks. 


\subsection{Network component characteristics}

The characteristics of particular network components will help to explain reactive power problems. The series reactance is usually within the range 0.05 to 0.15 p.u., depending on the transformer power rating, with low values for small transformers and high values for large ones [13]. The resistance is usually negligible. The total reactive power losses due to the magnetising shunt reactance $X_{i}$ of many small transformers within a distribution system can, however, be of some importance. The magnetising reactive power may also increase rapidly with the voltage level, due to core saturation.

Overhead lines and underground cables have their series resistances, series inductance and shunt capacitances distributed uniformly along their length.

If a constant operating voltages is assumed at the ends of the transmission line, the reactive power generated due to the capacitance (the charging reactive power), is practically independent of the power transmitted, while the reactive power loss due to the inductance varies with the power transferred.

A loss-less line (a reasonable approximation of a high voltage line) transferring an active power $P$ and with equal voltages at the line ends is in reactive power balance. The reactive power loss due to the line inductance is equal to the reactive power generated by the line capacitance. 


\subsection{Relationship between voltage and reactive power}

As regards to the terminal voltages of a transmission or distribution link, the link can be represented by the series impedance, if the shunt admittances of the equivalent circuit are included in the treatment of the connecting parts of the power system. The link may be an overhead line, an underground cable, a transformer or, possibly, a combination of such elements.

For transmission (not distribution) lines and cables, the reactance $X$ is usually much larger than the resistance $R$ and there will evidently be a much greater influence on voltage drop.

There are three major methods of power system voltage control [17]:

- Varying the excitation of the generators by means of their excitation systems.

- Varying the turn ratio of transformers by means of their on-load tap changers.

- Varying the shunt compensation, where applicable.

Shunt compensation means drawing or injecting reactive power at a particular point in a power system, by means of a shunt-connected device, which is installed for this purpose.

Drawing reactive power, e.g. absorption by means of a shunt reactor, affects the voltage reduction. Injection of reactive power, e.g. by means of a shunt capacitor. affects the voltage rise. The signs of the voltage changes mentioned arise because the network equivalent impedance has an inductive characteristic at the fundamental 
frequency. The shunt compensation may be fixed, switchable in steps or continuously controllable. The voltage changes around the nominal voltage when the shunt compensation is changed in a step-wise fashion.

Series compensation has been employed to a limited extent for reducing the voltage drop on sub-transmission and distribution circuits. By series compensation is meant partial compensation of the line series reactance, by means of a fixed capacitor in series with the line, thus reducing the effective reactance and the voltage difference [3].

Series compensation is mostly employed on long transmission lines. The main reason is to improve the transient stability or to obtain a desired load division among parallel circuits. At the same time, however, series compensation has a beneficial effect on the control of voltages and reactive power balance, because of the reduced variations in the network reactive power balances of the line [3].

Active power must, of course, be transmitted from the generators to the loads. Reactive power need not; and with regard to voltage differences, losses and thermal loading, it should not be unnecessarily transferred.

The subject of reactive compensation is easy to understand if a single link of a power system is considered, but quite complex when an entire power system is considered with its varied conditions and behaviours. 


\subsection{Reactions on the mains}

\subsubsection{Fundamental concepts}

Reactions on the mains can be described as the response of different types of distortions to the power system. The continually increasing number of electrical appliances with non-linear or non-stationary operating performance, like power units in electrical components, leads to more and more reactions which affect the voltage quality in public networks (Fig.2.2). These network reactions appear as voltage regulations, voltage asymmetries in three phase current networks, harmonic oscillations and intermediate harmonics $[1-3,18-20]$.

Network

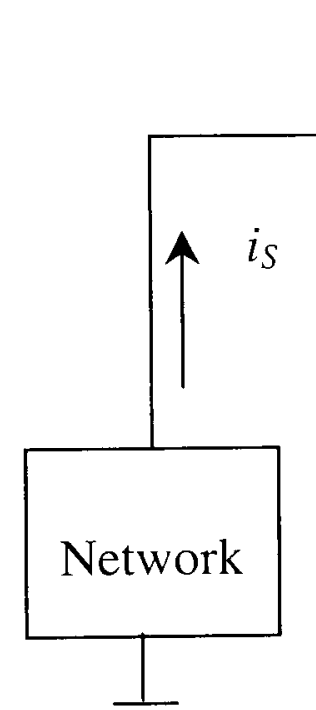

Load $P C C$

Network
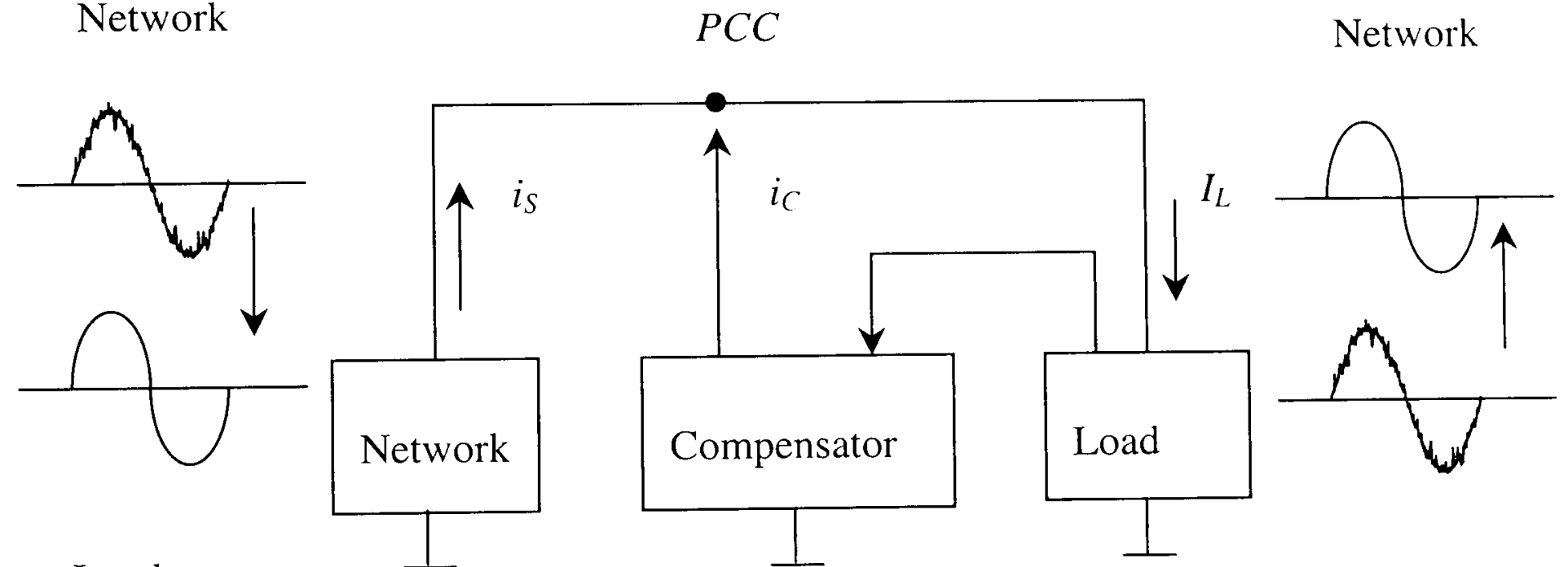

Load

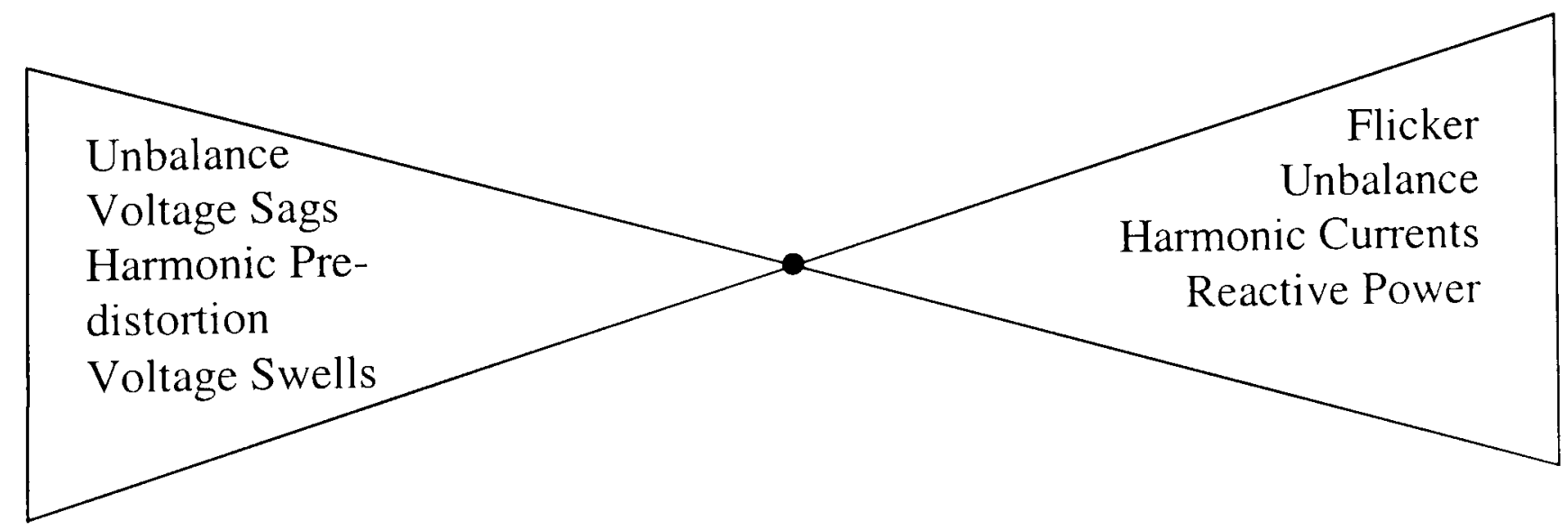

Fig. 2.2: Voltage Quality in Networks 
Network reactions lead to the following problems [10]:

- increased transmission losses

- worsening power factor

- impaired ground-fault neutralisation

- flickering

- disturbance of telecommunication networks

- effects on switchgear tripping

- malfunction of centralised control systems

- malfunction of electronic controls

In order to limit network reactions, regulations are being imposed which will stipulate the limiting values of appliances and determine permissible interference $[10,11]$. The network reactions (Fig. 2.3) of an electrical system increase with the rated connected power at the PCC, $S_{\text {Comnected }}^{\text {Rated }}$, and fall with the short-circuit capacity $S_{s c}$, which is dependent on the capacity of the transformer used and the distance of the PCC from the local network station. For the permissible interference rating, the maximum system capacity and the minimum short-circuit capacity must always be applied. Network reactions are determined by the following factors [10]:

- short-circuit capacity at the PCC

- system reference power

- maximum capacity of system component

- system assembly

- existence of several facilities at the PCC and supply network

Fig. 2.4: shows the influences of harmonics on the mains and their remedies in power systems. 


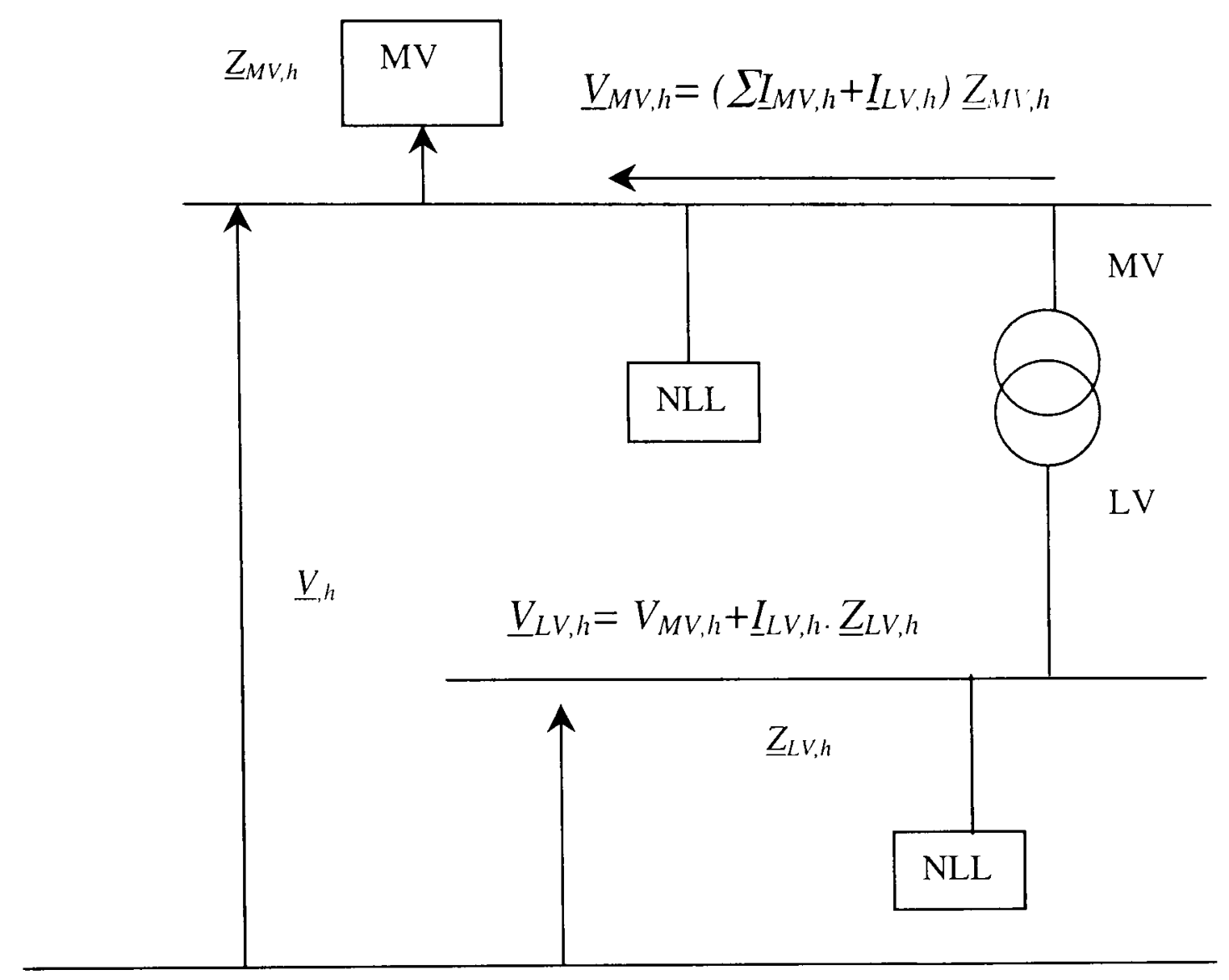

Fig. 2.3 Reactions on the mains

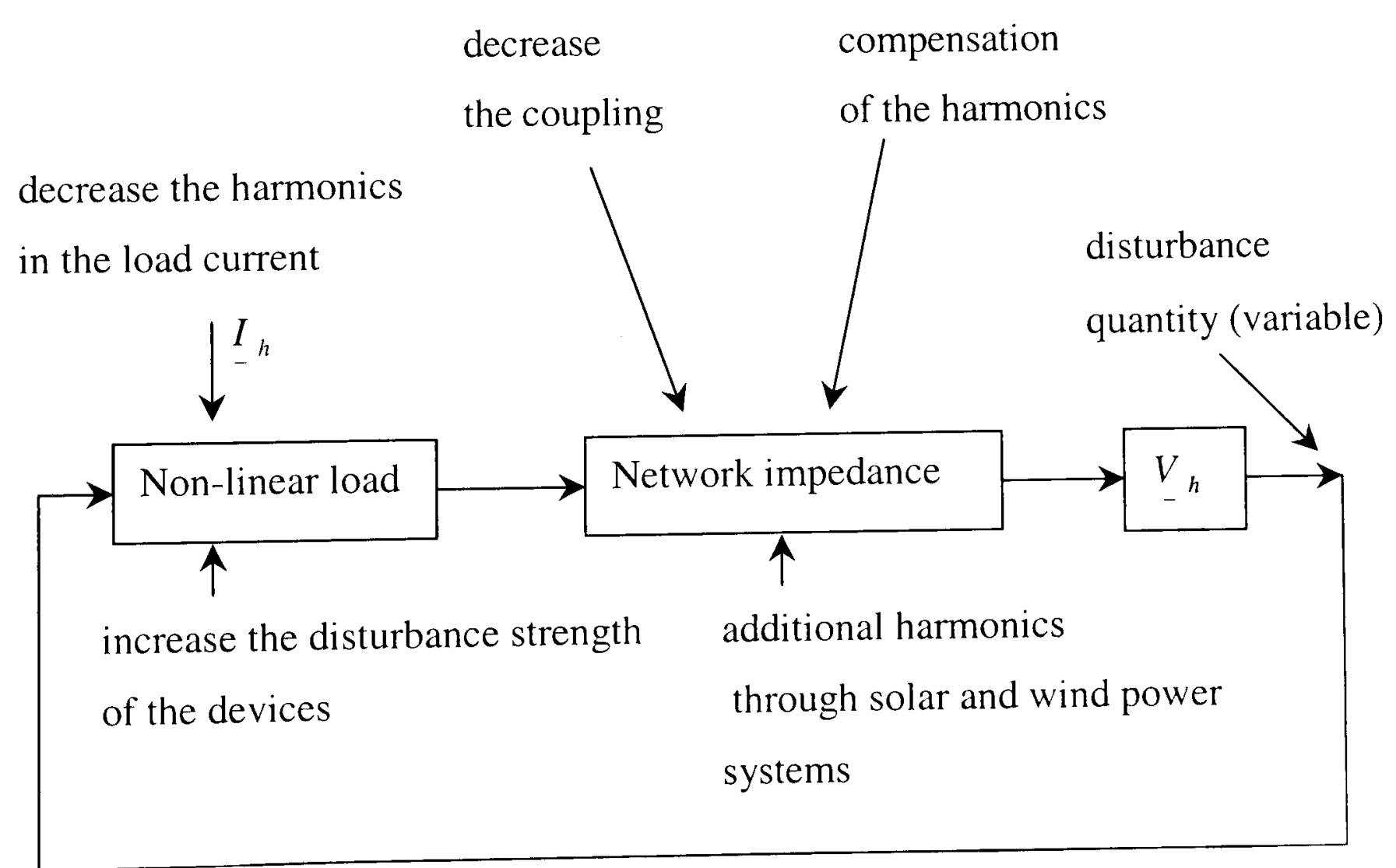

Fig. 2.4: Influences of harmonics and their remedies in power systems 


\subsubsection{Voltage asymmetries}

Asymmetric loads on the three conductors of a three-phase network cause asymmetric currents, and thus asymmetric voltages in the supply network [13]. At the same resistive rating as a single-phase load in a low-voltage network, the current load of electrical equipment in a three-phase network can be three times higher than the asymmetric load. If the single-phase apparent power, $\mathrm{S}_{\mathrm{sp}}$, does not exceed $5 \%$ of the connecting power $S_{\text {Connected }}$ of a consumer system, the effects of asymmetric loads can be neglected. The maximum acceptable impairment of the electrical equipment of other consumers determines the upper limit on degree of voltage asymmetry $[21,22]$.

The degree of asymmetry is defined as the ratio of voltage in the positive sequence system to the voltage in the negative sequence system of symmetric components. Consequently, the asymmetric load of a system should not cause a higher degree of voltage asymmetry than $(\mathrm{T}<10 \mathrm{~min})$ at the $\mathrm{PCC}$. To balance the resistive load, a susceptance must be added from the loaded to the unloaded conductors. The necessary compensating elements can be determined by means of the symmetric components (Fig.2.5).

\subsubsection{Voltage fluctuations}

Insignificant voltage fluctuations due to pulsating or considerably oscillating power input or output can cause disturbances in connected equipment and facilities, particularly annoying flickering in lamps. A dynamic compensation system (Fig. 2.6) is permitted if it can be proven that the following conditions are met at the PCC $[10,21,23]$ 
1. Compensation of the reactive power

2. Stabilisation of voltage on the low voltage side

3. Stabilisation of voltage on the medium voltage side

$$
L_{1} \quad L_{2} \quad L_{3}
$$

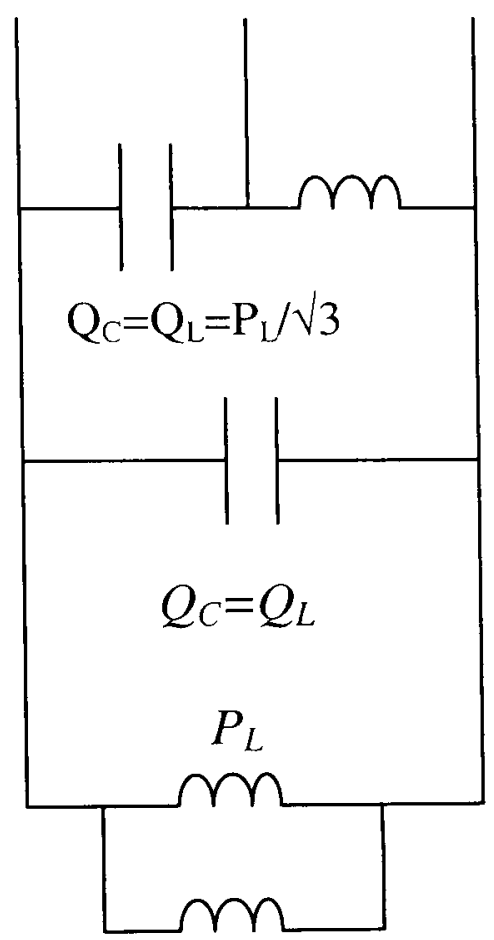

$Q_{L}$
$L_{1}$

$L_{2}$

$L_{3}$

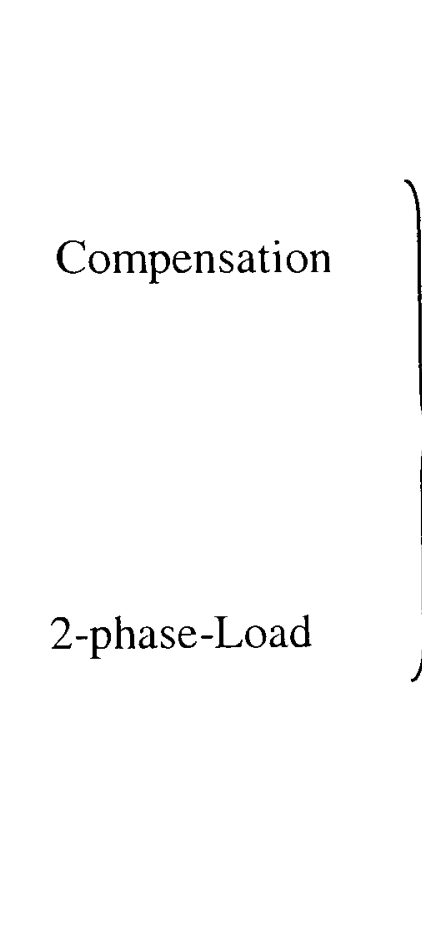

Fig.2.5 Schematic diagram of the voltage Asymmetries

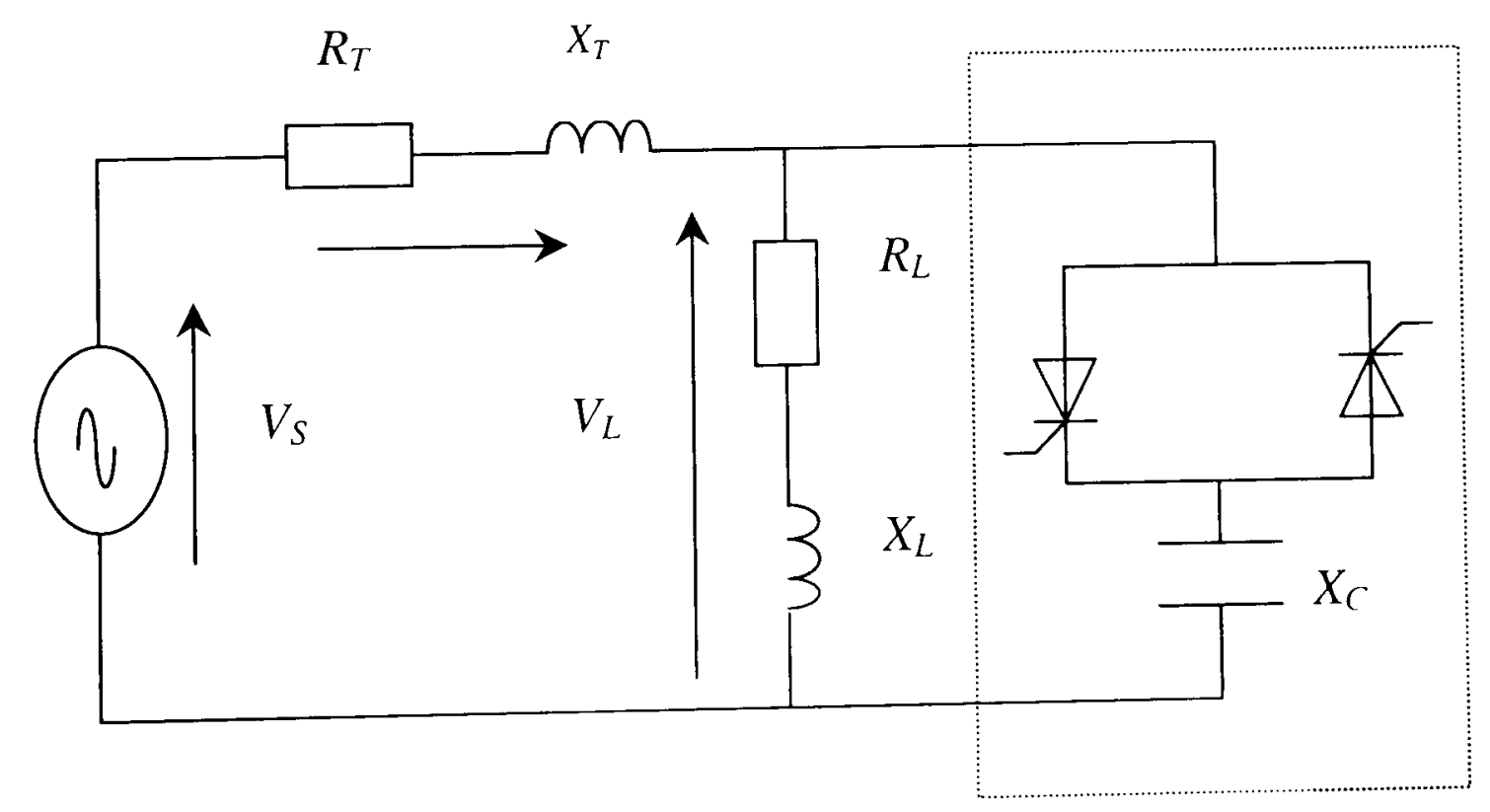

Dynamic Compensation

Fig: 2.6 Schematic diagram of the Dynamic Compensation 


\subsubsection{Harmonic oscillations}

Harmonic currents will produce higher harmonic voltages in the impendances of the Supply network, which interfere with the $50 \mathrm{~Hz}$ network voltage [10]. If these higher harmonic voltages surpass the resistance of the connected electrical equipment, the service life of capacitors and motors will be considerably diminished due to thermal overload. Permissible interference is stipulated in the regulating standards. Only systems, which comply with the stipulated maximum harmonic currents, are allowed.

\subsubsection{Voltage drop in the networks}

Essentially, the voltage rating is determined by the product of power reactance and Reactive power. Reactive power compensation thus improves voltage maintenance in the network [10].

\subsubsection{Voltage distortions}

Non-linear load in a sinusoidal voltage generates harmonic currents, which lead to voltage drops in the internal impedance of the system and to the distorted supply voltages $[10,15,16]$.

\subsection{Reactive power compensation in transmission systems}

Well-planned and co-ordinated reactive power compensation is an indispensable element in the design and operation of a reliable power system. The effectiveness of ractive power control on a power system may be of the utmost importance not only' under normal conditions, but also during major system disturbances. 
It is often advantageous to operate the transmission parts of a power system [5.24]:

- With a fairly flat voltage profile, in order to avoid unnecessary reactive power flows

- With relatively little supply of reactive power into the distribution systems

- With reactive power capacity reserves available for use in connection with major disturbances and under generator, transformer or line outage conditions A detailed discussion of the above is presented in the following five sections.

\subsubsection{Steady-state voltage control}

The aim of steady-state voltage control is to keep the transmission bus voltages within narrow limits, while the load transferred varies. The desirable voltage range could be a few percents around a chosen nominal voltage. The set voltages of the different buses should always be such that the reactive power flows are minimised.

Since the active power transmitted may vary greatly hour by hour, the variation in the reactive power balance of a line may be considerable. In the case of a long transmission, where variations of hundreds of MVARs per line are involved, this has a large influence on the reactive power balance of the entire transmission system. If there is an outage, either forced or scheduled, of one line out of a number of heavily loaded parallel lines, a great increase in reactive power demand may be created. The line generation of reactive power is reduced and the line consumption of reactive power is greatly increased [25].

Lalrge generators provide the basic voltage control of a power system. each having its own excitation system with an automatic voltage regulator. The generators are used 
for voltage control at the terminals to which they are connected. Reactive power is generated or absorbed, depending on the load connections. Transfer of reactive power from the generators to electrically remote points of the power system, or vice versa, is usually avoided under normal operating conditions. Generators are, however, very important as reserve sources of reactive power, even when it is sometimes needed from the generators, e.g. after contingencies such as the sudden loss of a main generator or a major line section. The short-time reactive overload capability of generators may also be valuable on such occasions. In addition, pure synchronous condenser operation of generators can be valuable under unusual system operating conditions.

Large generators are usually connected directly to transmission networks via step-up transformers. The terminal voltage of a large generator is usually controlled within $5 \%$ range around the nominal voltage at rated load. Under light load conditions on a long transmission line, the excessive line-generated reactive power must be drawn out at the buses in order to keep down the voltages. Shunt reactors are frequently used on transmission line [26].

They are also needed on shorter lines, if these are supplied from weak systems. There is a trend towards the highest degrees of compensation for the highest system voltages and the longest lines.

Shunt reactors in transmission systems are usually connected either to tertiary windings of transformers, for instance at $10 \mathrm{kV}$, or direct at line potential; in a few cases they may be connected to generator buses. Nowadays, most new transmission 
system reactor installations are at line potential. Shunt reactors are also used in $\mathrm{HV}$ transmissions with long lines or cables [3].

By increasing the load transferred through a long transmission line, the excessive linegenerated reactive power decreases and reactive power absorption has to be reduced. At least some of the shunt reactors are usually disconnected so as not to cause an unnecessary voltage drop.

Transmission lines with series compensation, which can be much more heavily loaded, have lower reactive power losses because of the series reactance compensation. This results in a smaller variation in the reactive power balance. The reactive power injections, which may be needed under heavy load conditions, are supplied from generators, synchronous condensers, thyristor-controlled static compensators and shunt capacitors. Shunt capacitors, however, are not often used in high voltage transmissions [3]. They are normally found in some transmission substations, especially those feeding into sub-transmission or distribution systems, and also within sub-transmission systems. In cases where the voltage has to be better controlled than is possible with breaker switched shunt reactors or shunt capacitors, the synchronous condenser has been the traditional means of absorbing or generating reactive power. Synchronous condensers are in use $[3,25]$ :

- at the receiving end of long radial transmissions

- at main buses within a meshed transmission network with long lines, particularly in regions where there is only little generation 
For new installations in such cases, a thyristor-controlled static compensator will be used in preference to a synchronous condenser because of lower costs. This will lead naturally to superior voltage control.

\subsubsection{Reduction of temporary over-voltages}

Fundamental frequency over-voltages originate from switching operations and faults. If receiving end load-dropping occurs from a high load condition, leaving the line energised from the sending end only for a short period of time, a high fundamentalfrequency over-voltage will appear at the receiving end. The voltage rise is due to the change from active/inductive load to capacitive load - the so-called Ferranti effect. If the load dropping brings about a separation of systems, problems of frequency rise and of generator self-excitation may appear. The worst case is a single line-to-ground fault at the receiving end followed by load dropping. Fundamental-frequency overvoltages, as discussed here, are usually most critical during the initial period of transmission development, when the short-circuit capacities and the number of interconnections are low [10].

The remedy for fundamental-frequency over-voltages, if they constitute a major problem for the particular system, is reactive power consumption. The shunt reactors installed for the steady-state voltage control and the line energisation are usually sufficient. There is, however, a problem in that some or all of the reactors may be disconnected during heavy loading conditions. 


\subsubsection{Synchronous stability improvement}

The term synchronous stability denotes the ability of a power system to retain the synchronous machines in synchronism without sustained rotor oscillations, both during steady-state conditions, (steady-state stability) and after large disturbances (transient stability). By the term stability limit is meant the maximum power which can be continuously transmitted while maintaining a stable condition $[15,16]$.

The critical transient stability limit is usually lower than the steady-state stability limit. From the economic point of view, transient stability, and in particular the firstswing transient stability, is the most important type of stability, because it may influence the choice of high-power elements in the power system. The resistance can be disregarded in this respect. The voltage-angle difference of the link is determined under both steady-state and transient conditions. Without going into a discussion of the transient stability as such, which lies outside the scope of this thesis, the following statement is made: there is a strong interaction between the transient stability and the voltage-angle difference. In critical cases of transient stability of long transmissions lines (not all cases are critical), it is important to note that the contribution of to the total voltage-angle difference of the generators and the transmission system does not become too large. Both shunt compensation and series compensation can be used to reduce it, thereby raising the transient stability limits $[27,28]$. If a severe stability criterion is applied, such as a three-phase short-circuit or a double-line to ground fault, the first-swing transient stability may be critical and of main concern. Full advantage should, of course, be taken of low-cost countermeasures such as rapid fault clearing or rapid re-closing, but in critical cases this is usually not sufficient. Shunt reactive power injection by static compensator can be used. Due to the depressed 
voltages during the first power swing, the large nominal size of the compensators needed will generally make this method much less attractive than series compensation. Series compensation is, in many cases, the most cost-effective method of increasing the first-swing stability limit [29].

If a minor stability criterion is applied, such as a single-line-to-ground fault. the damping of the subsequent electro-mechanical oscillations between generators may be of more concern than the first-swing stability. Oscillations may build up and lead to instability. In the network they show up as power and voltage oscillations. Damping might, of course, be of concern in cases of severe disturbance. The thyristorcontrolled static compensator constitutes an excellent means for further improvement in damping these oscillations. How the compensator should be controlled depends on the network configuration - where the loads are connected. In some cases a certain degree of damping can be achieved by keeping the voltage constant. In many cases the optimum damping effect will be achieved by controlling the compensator output in a proper relationship to oscillating active power [25].

\subsubsection{Prevention of voltage collapse}

Voltage collapse means a severe voltage depression without inherent recovery. The voltages do not decrease to zero but to low values, making continued proper operation, of a single load in the simplest case, impossible. This phenomenon may appear in both transmission and distribution systems. Generally, less attention is paid to voltage collapse than to synchronous instability, but it can be of great importance in particular cases. The key cause of a voltage collapse is inadequate reactive power gencration. One or more of the following factors are usually involved [17.23.25]: 
- High transfer reactance

- Line outages

- Insufficient reactive power generation reserves

- High load content of induction motors

- Automatic transformer tap-changing

- The actions of excitation limiters on synchronous machines

Voltage collapse can usually be prevented by installing sufficient reactive power generation sources, e.g. breaker-switched shunt capacitors when slow voltage collapse can be expected, and thyristor-controlled static compensators in cases of rapid voltage collapse [20].

\subsubsection{Extensive cable networks}

Cables produce more reactive power than overhead lines. This creates voltage and reactive power control problems in some industrial areas with extensive underground transmission cables, particularly during light loading periods. Local generators and shunt reactors are used in the first place to absorb the excessive reactive power.

It is possible that both shunt reactors and static compensators of pure thyristorcontrolled reactor type will be installed in the future to meet the needs for reactive power absorption in transmission cables; the latter is proposed due to the superior voltage control features [30]. 


\subsection{Reactive power compensation in distribution systems}

Distribution systems need to be supplied with reactive power to equalise the reactive power consumption of the loads and the reactive power losses of the distribution network itself. The required reactive power is supplied through one or more of the following sources $[23,31,32]$ :

- Transmission system

- Synchronous machines within the distribution system

- Shunt capacitors

- Static compensators

Many electricity supply utilities are, for the reasons discussed in the previous sections, somewhat restrictive in supplying reactive power from transmission to distribution systems. This is often reflected in the supply tariffs to large consumers, such as retail distribution utilities and high-power industrial customers, with a penalty for low power factor. Under normal steady-state conditions, the voltage at the consumer terminals should lie within a given range around the nominal voltage. The term power-factor correction is used in conjunction with slowly varying loads on distribution systems. It usually refers to the method of generating reactive power relatively close to the loads consuming it.

Power-factor correction by means of fixed and switched shunt capacitors is often used in urban, residential and rural systems, and extensively in high-power industrial systems [21,33]. The objective is usually one or more of the following:

- To reduce power costs by avoiding a low power-factor, if applicable.

- To reduce active $\left(I^{2}, R\right)$ and reactive $\left(I^{2}, X\right)$ power losses in the distribution networks. 
- To increase current capacity of transformers and cables (and, possibly, overhead lines).

- To increase voltage levels and, in the cases of switched shunt capacitors, to improve voltage regulation (to reduce voltage variation from light to peak load conditions).

In many existing systems the two latter points can postpone or even eliminate large investments in new equipment. An evaluation of the above gains versus the shunt capacitor costs has to be made, considering different constraints such as maximum size of switched banks with regard to voltage change and space availability [34].

The control of switched shunt capacitors is an area where many methods are in use: manual, time-switch, automatic regulator or relay control of voltage or current, or reactive power, or power factor. There are many different configurations (Urban, Residential and Rural Systems) of switched capacitors used to control reactive powers and voltages for these systems. One simple example: a 110/20(10) kV distribution substation transfers power from a $110 \mathrm{kV}$ transmission to $20(10) \mathrm{kV}$ distribution feeders (primary circuits). Each distribution feeder supplies a number of 20(10)/0.4 $\mathrm{kV}$ distribution transformers, each of which supplies consumer feeders (secondary circuits) at the utilisation voltage [21].

The voltage level and voltage regulation (voltage drops) are usually considered when dimensioning the distribution circuits. Voltage control is actuated by means of onload tap changers on the distribution substation transformers. Often, as the load increases, the controlling device raises the substation secondary voltage to compensate for the increased voltage drops in the distribution feeders [29]. 
Shunt capacitors are much used in these distribution systems; in addition they serve for voltage control [27]. In spite of the difficulties in giving generally applicable rules, the following summarises are to be considered in locating shunt capacitors in these types of distribution system:

- Locate the shunt capacitors as close to the loads as possible.

- In the first place, install shunt capacitors, which can postpone an otherwise necessary reinforcement of the network.

- Secondly, install low-voltage (utilisation voltage) fixed shunt capacitors in such a way that they compensate for the yearly minimum reactive load of the system.

- Meet the remaining need by installing switchable shunt capacitors: in the first instance, low-voltage banks at large customers and medium-voltage banks at intermediate switching stations.

\subsection{High-power industrial systems}

Many major industrial plants purchase power at 20,10 and $6 \mathrm{kV}$ or above. The distribution systems usually have at least two lower voltages: a medium voltage, e.g. $10 \mathrm{kV}$, for primary distribution and large loads, and a low voltage, e.g. $0.4 \mathrm{kV}$, for small loads.

\subsubsection{Supplying Steady-state reactive power and voltage control}

Induction motors are common loads, which consume reactive power, at a rate of 0.6 to 1.1 reactive to active power at rated output. Static power converters and uncompensated fluorescent lamps are other examples. Static power converters for 
rolling-mill DC motors and arc furnaces, in steel mills, have reactive power consumption with a large average value and subject to substantial, rapid fluctuations $[21]$.

Primary voltage control is usually achieved by means of on-load tap changers on the step-down transformers from the metering point [35]. Existing synchronous machines are also used for reactive power supply and voltage control, synchronous motors for reactive power supply. In a few cases, existing small synchronous condensers may possibly still be used [29]. Power-factor correction by means of fixed and switched shunt capacitors is in extensive use in industrial systems [27].

Static power converters and arc furnaces produce current harmonics, which must be considered during planning and design of shunt capacitor installations. In many cases, shunt capacitors are arranged for both reactive power production at the fundamental frequency and filtering of current harmonics [34].

\subsubsection{Reduction of voltage fluctuations}

Rapidly fluctuating loads create voltage fluctuations, which may cause annoying disturbances, particularly flicker of filament lamps in adjacent load areas. The most pronounced load of this kind is arc furnace, which is usually a large load connected directly to the power system [21].

It is characterised by:

- Low power factor

- Imbalance of phase currents 
- Rapid, large active and reactive power fluctuations of more or less random character

- Current harmonics

The mean reactive power consumption can be compensated for by means of a shunt capacitor. The voltage fluctuations remain, however, and are even somewhat magnified by the shunt capacitor at the arc furnace bus. The Point of Common Coupling (PCC) with other consumers is the critical one with regard to voltage fluctuations. Efficient reduction of voltage fluctuations means that it is possible to operate the arc furnace at a higher average voltage level without adverse effects, thus increasing the furnace active power and reducing the melt-down time. This was overlooked at first, but it later became an economic incentive for the installation of static compensators. Suitable types of thyristor-controlled static compensator are thyristor-switched capacitors operated in small steps as well as fixed capacitor in parallel with a thyristor-controlled reactor.

\subsubsection{Reduction of voltage drop during large motor starts}

Direct on-line starting is the simplest, most straightforward and cheapest of all the starting methods for induction motors. It creates, however, a high inrush current at low power factor, $\cdot$ which in turn causes a voltage drop. In cases where there are one or more large motors (in relation to the network short-circuit capacity), these voltage drops may be objectionable depending on their size and frequency of occurrence. They may disturb the performance of other loads in the plant and the loads of other consumers. In cases of very large starting load torque, the starting time of the motor itsclf might be critical [35]. One method of reducing the voltage drop is to use a starting shunt capacitor, which operates only during starting. A technically much 
superior method is the use of a static compensator. Other application areas where static compensators could be used for this purpose are the auxiliary supply system of large thermal power stations and very large pumping motors fed by long subtransmission or distribution feeders.

\subsection{Reactive power compensation devices}

A synchronous generator produces reactive power when over-excited and absorbs reactive power when under-excited. The reactive power output is continuously controllable. The step-response time in voltage control is from several tenths of a second upwards, depending on various factors.

The rated power factor of generators usually lies within the range 0.80 to 0.95 . Generators installed remotely from load centres usually have higher power factors; this is often the case, for example, with large hydro-electric generators. With regard to severe outage conditions, it is not justified to choose a low power factor when installing a large steam-turbine generator close to load centres [3].

The shunt reactor, the shunt capacitor, the synchronous condenser and the static compensator are possible shunt compensation devices. These are discussed in the following sections.

\subsubsection{Shunt reactors}

A shunt reactor is a reactor connected in shunt with a power system for the purpose of absorbing reactive power [13]. In cases where voltage control requirements allow a fixed or mechanically switched shunt ractor to be used. it is usually the most 
economic means available for reactive power absorption. The majority of shunt reactors are used in conjunction with long overhead transmission lines. They are also used with high voltage and transmission underground cables in large urban areas.

Shunt reactors in use range in size from a few MVARs at medium voltages up to hundreds of MVARs with overhead transmission lines. Both single-phase and threephase transmission reactors are used, with a tendency to prefer single-phase reactors.

\subsubsection{Shunt capacitors}

A shunt capacitor is a single capacitor unit or, more frequently, a bank of capacitor units connected in shunt to a power system for the purpose of producing reactive power. In cases where voltage control requirements allow a fixed or mechanically switched shunt capacitor to be used, it is usually the most economic means available for reactive power production. The majority of shunt capacitors are applied within different types of distribution system: industrial, urban, residential and rural, where they find widespread use for power-factor correction [2]. Some shunt capacitors are installed in transmission substations. Shunt capacitors in use range in size from single units rated at a few KVARs at low voltage up to banks of units rated at hundreds of MVARs on transmission lines. The construction of a shunt capacitor bank is very flexible, since it is built up from modular components. The smallest primary component is the capacitor unit. This consists of capacitor elements connected in parallel-or in series. A bank usually consists of capacitor units connected in both parallel and series. Outstanding features of shunt capacitors are their low overall costs and their flexibility in application. An unfavorable characteristic. most important in connection with major outages and disturbances. is that they provide the least support 
at the very time when it may be most needed, because the reactive power output is proportional to the voltage squared. If used in a proper mix with other reactive power sources, however, this is no obstacle to an extensive use of shunt capacitors. The losses of modern shunt capacitors are of the order of 0.2 to $0.6 \mathrm{~W} / \mathrm{KVAR}$, including the losses of fuses and discharge resistors [3].

\subsubsection{Synchronous condensers}

A synchronous condenser is an idle-running synchronous motor, used for the generation or absorption of reactive power. Synonymous terms are synchronous compensator and synchronous phase modifier. The synchronous condenser is the traditional means of continuous reactive power control. Synchronous condensers are used in transmission systems: at the receiving end of long transmissions, in important substations and in conjunction with HVDC inverter stations. Small synchronous condensers have also been installed in high-power industrial networks in steel mills; some of these might still be in use today. Synchronous condensers in use range in size from a few MVAs up to hundreds of MVAs. The rated voltage usually lies below 24 kV. Modern synchronous condensers are generally equipped with a fast excitation system with a potential-source rectifier exciter. The size of a synchronous condenser refers to the continuous MVA rating for the generation of reactive power. In the generating mode of operation it usually has a fairly high short-time overload capability. The absorption capability is normally of the order of 60 percent of the MVA rating, which means that the control range is usually 160 percent of the MVA rating $[3,13]$. The reactive power output is continuously controllable. The stepresponse time with closed-loop voltage control is from a few tenths of a second 
upwards, depending on various factors. The active losses of hydrogen-cooled synchronous condensers are of the order of $10 \mathrm{~W} / \mathrm{KVAR}$ at rated output [3].

In recent years, synchronous condensers have been largely superseded by thyristorcontrolled static compensator in new installations, due to cost, performance and reliability benefits. One exception is HVDC inverter stations, in cases where the short-circuit capacity has to be increased. The synchronous condenser can do this, but not the static compensator.

\subsubsection{Thyristor-controlled static compensators}

A thyristor-controlled static compensator is a static shunt reactive device. The reactive power absorption or generation of which can be varied by means of thyristor switches. Because it is a relatively new means of reactive compensation, it will be described and discussed in greater detail than the other devices. The term static compensator covers not only the thyristor-controlled compensator but also other types and, in particular, the self-saturated reactor type. Even though the self-saturated reactor compensator was introduced before the thyristor-controlled one, the latter completely dominates the market for transmission applications, making up more than 95 percent by MVAR of all compensators ordered [36-39]. Today, it is also dominant in highpower industrial applications. Two types of thyristor-controlled elements are used in static compensators: the thyristor-switched capacitor (TSC) and the thyristor- (phaseangle-) controlled reactor (TCR).

From a fundamental frequency point of view, both can be considered as a variable ractance, the former being a variable capacitive reactance and the latter a variable 
inductive reactance. Switching off a capacitor is accomplished by suppression of the firing pulses to the thyristors so that they will block as soon as the current becomes zero. In principle, the capacitor will then remain charged to the positive or negative peak voltage and be ready for a new transient-free switching on.

\subsubsection{Thyristor-switched capacitor compensator}

Static compensators of the thyristor-switched capacitor type (Fig.2.7) are characterised by $[6,8,38-40]$ :

- Stepwise control

- One half-cycle average delay (maximum one cycle) in executing a command from the regulator, as seen for a single phase system

- No annoying transients

- No generation of harmonics

- Low losses at low compensator reactive power output

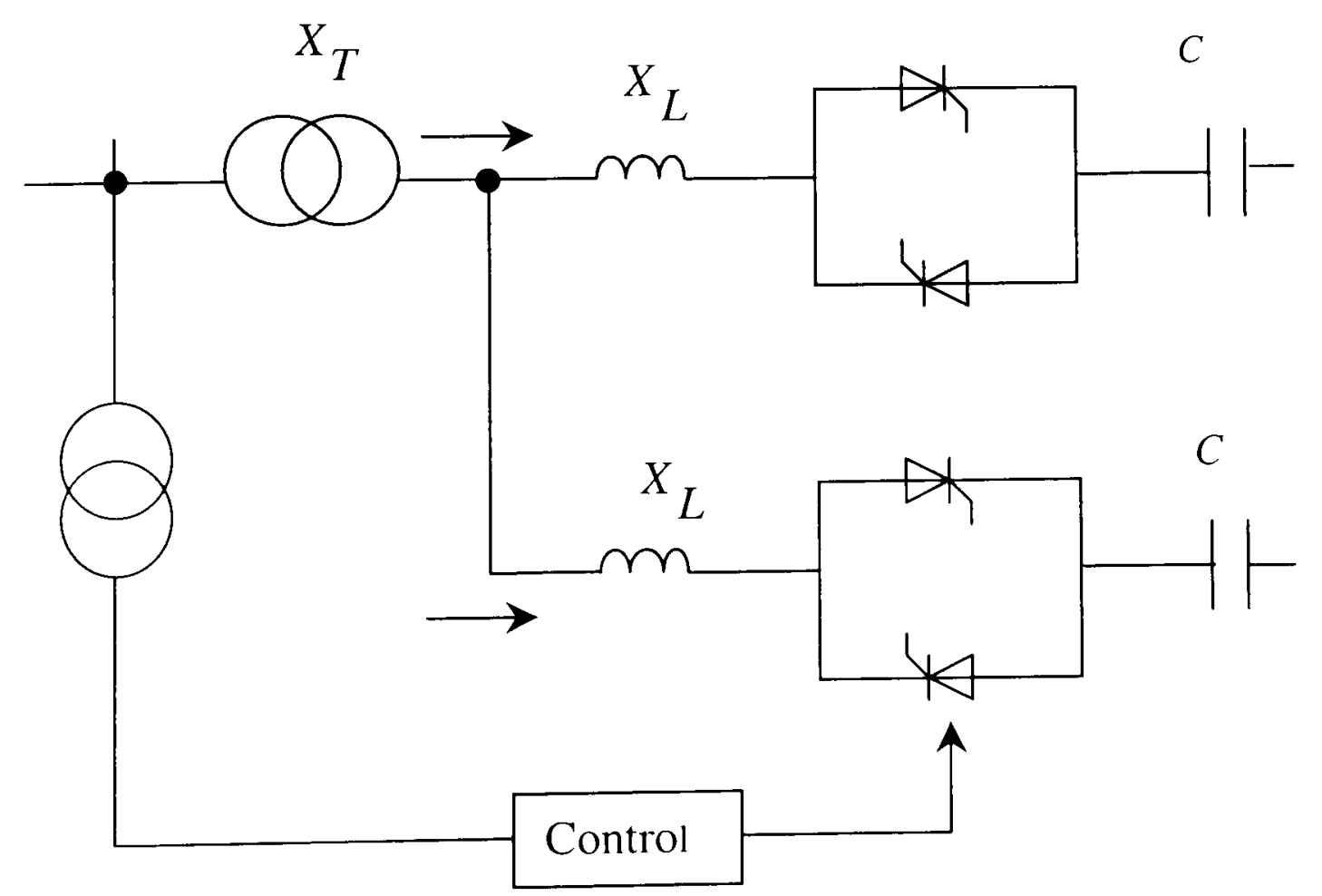

Fig. 2.7 Tyhristor-switched capacitor compensator 


\subsubsection{Thyristor-controlled reactor compensator}

Static compensators of the thyristor-controlled reactor type (Fig. 2.8) are characterised by $[6,38-41]$ :

- Continuous control

- Maximum delay of one half-cycle delay in executing a command from the regulator, as seen for a single phase system

- Practically no transients

- Generation of harmonics

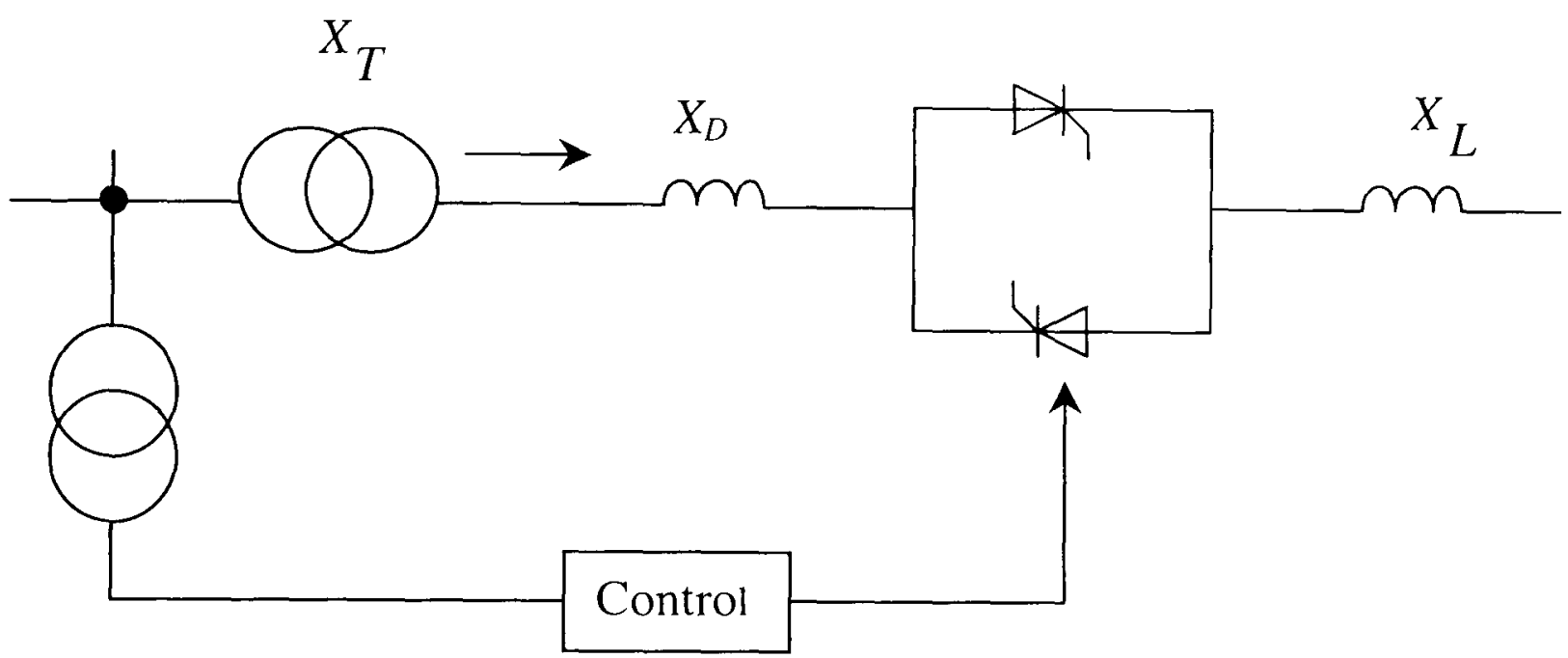

Fig. 2.8 Tyhristor-controlled reactor compensator

Irrespective of the reactive control range required, any static compensator can be built up from one or both of these elements and, if required, combined with an ordinary shunt capacitor bank or shunt reactor, fixed or breaker-switched.

A compensator arrangement of a few large steps of thyristor-switched capacitors and one or two thyristor-controlled reactors is very attractive for many transmission applications. It combines the favourable properties of the two thyristor schemes discussed. 
The compensator gives continuously variable reactive output from full lagging to full leading compensation. Advantages compared with other arrangements are: low losses, reduced harmonic generation and the ability to rapidly control the capacitor part. Most transmission applications require closed-loop bus voltage control by an automatic voltage regulator. Within the compensator operating range, and under optimum conditions, the response time is of the order of two to three cycles of the network voltage, including the VAR compensation.

\subsection{Series capacitors}

A series capacitor is not a reactive compensation device but a reactance compensation device. Since series capacitors (Fig.2.9) greatly influence the reactive power conditions of transmission systems, it is, however, proper to include them in the description of reactive compensation devices. Series compensation is mainly installed in high voltage transmission systems for one or both of the following two reasons. The first is to increase the transmission load capability as determined by transient stability limits. The other reason is to obtain a desired load division among parallel circuits. Furthermore, series capacitors influence the control of both the voltages and the reactive power balance. Series capacitor range is between 100 and 800 MVARs [13]. The choice of location is important for economical reasons and system reliability, and should take into account voltage profiles, compensation efficiency. losses, fault currents and over-voltages. Most of the compensation devices can also be connected together. Shunt reactors are connected to $400 \mathrm{kV}$ busses or generator busses. Most shunt capacitors are to be found within distribution systems, including industrial plants [3]. 

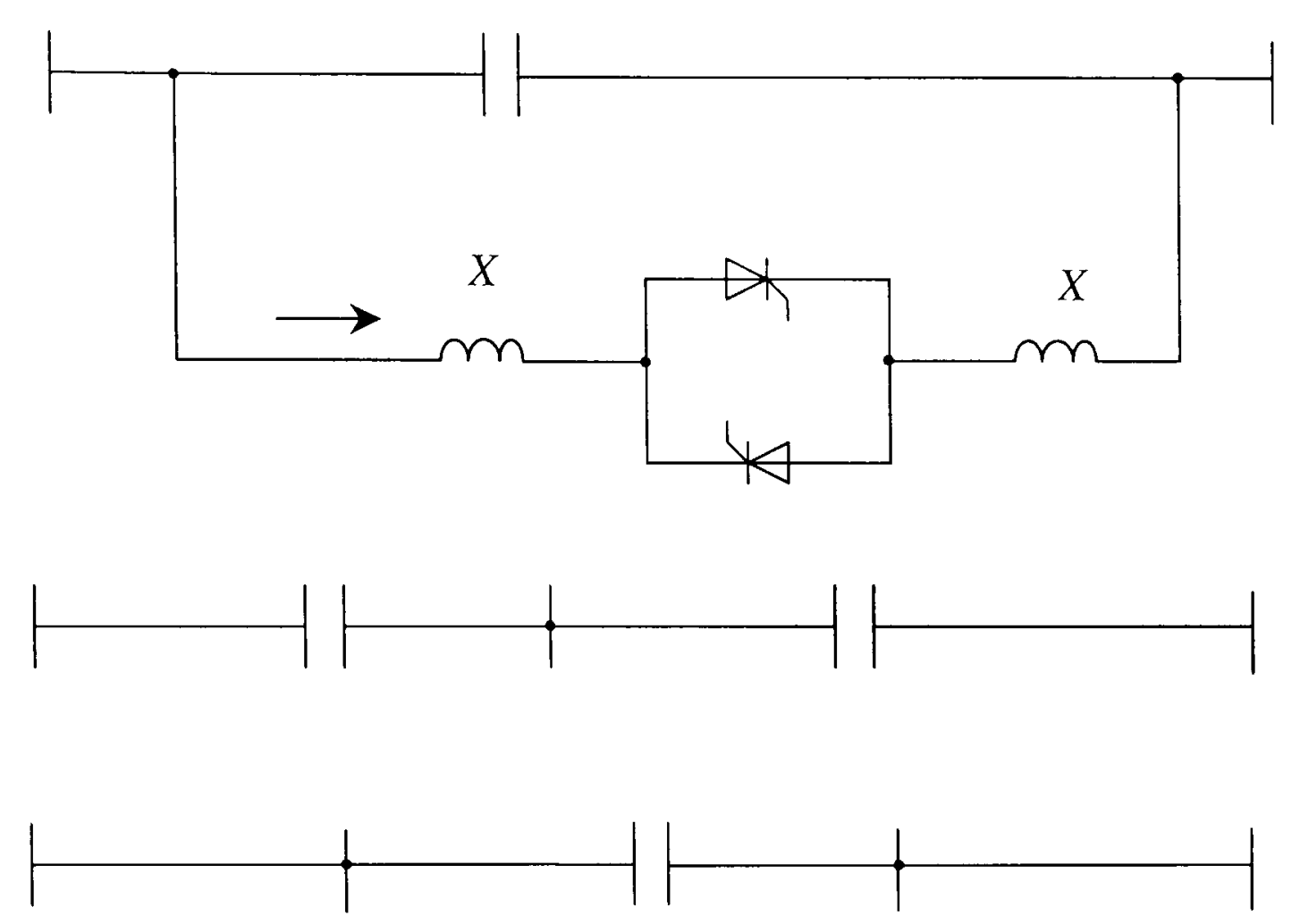

Fig. 2.9 Series capacitor

\subsection{Passive filter circuits}

Passive filters use L-C tuned components, which eliminate the unwanted harmonics in the system [1-5]. Fig. 2.10 shows the most common filter circuits. Single-tuned $1^{\text {st }}$ order filters are mostly used in the $220 \mathrm{~Hz}$ range [4]. In cases with high harmonic currents, high-pass damped filter circuits can be used. Double filters are applied in the case of low reactive power/high supply voltage. Passive filters are easy to design but they may produce resonance within the power system.

\section{Advantages of passive filters:}

1. easy to design

2. reliable operation

3. can be used in filter banks

4. very cheap configuration

5. acts as power factor improvement devices 


\section{Disadvantages of passive filters:}

1. depend on system impedance

2. tuned only for one harmonic frequency

3. resonance may occur

4. affected by capacitor ageing

5. has large number of passive components

6. Bulky

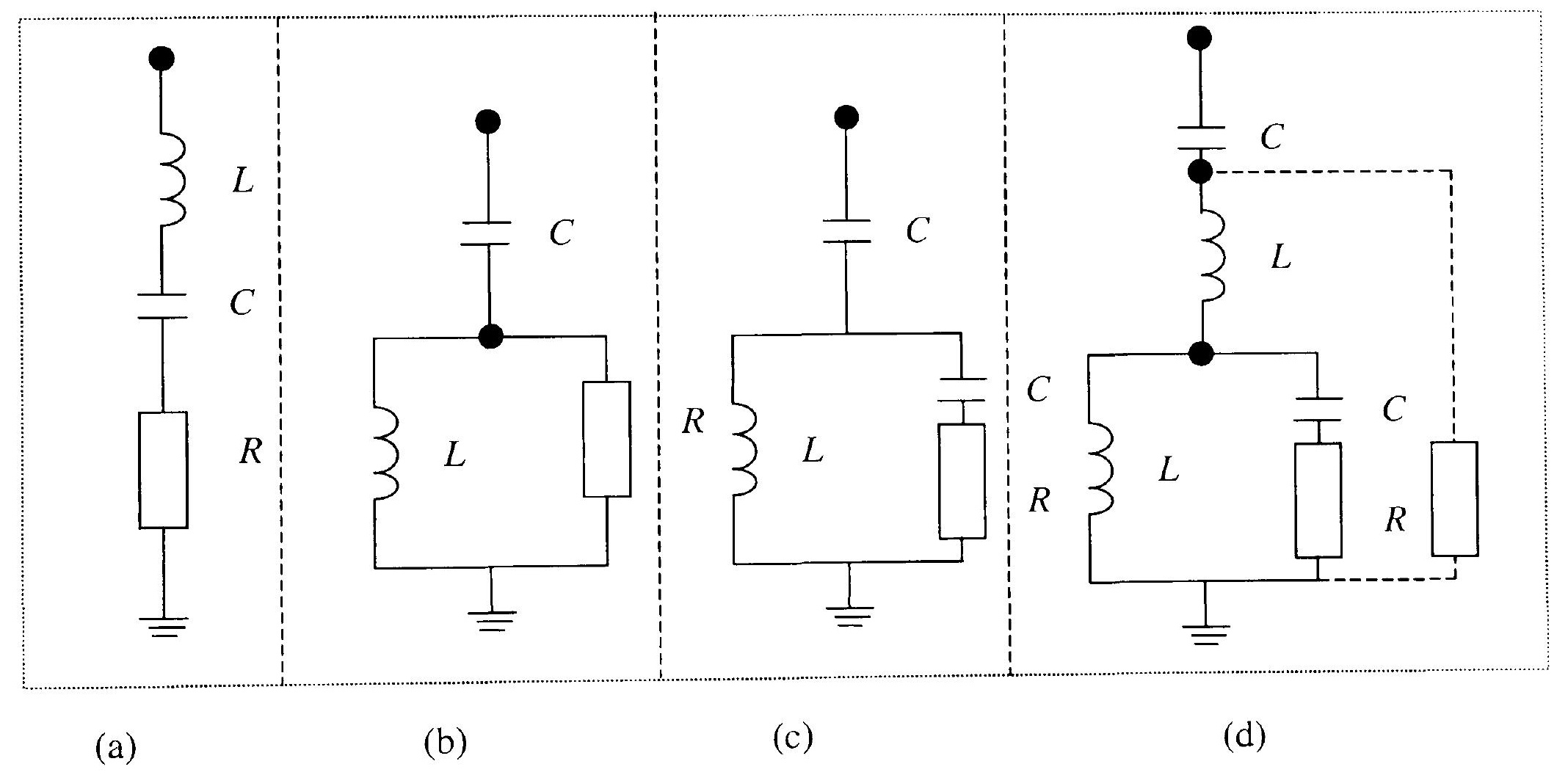

Fig. 2.10 Passive filter circuits
a) Single tuned $1^{\text {st }}$ order filter
b) High pass damped $2^{\text {nd }}$ order filter
c) High pass damped $3^{\text {rd }}$ order filter
d) Double filter 


\subsection{Shunt Active power filter}

Based on its application, the literature and published papers, it can be said that active filtering has played a major role in reactive power compensation and harmonic elimination. Over the last thirty years, research has focused on active filtering circuit configurations and control systems. This chapter gives an overview of the classification of these techniques under the following subdivisions [9,42].

1. Power rating and its dynamic response for low and medium voltage power applications elements [44-47].

2. Power circuit configuration, such as shunt, series and other combinations of these circuit elements $[6,8,9,38-40,42-64]$.

3. The compensated variables for the filtering techniques, i.e. harmonic compensation, VAR-compensation and balancing-three-phase-systems [6,4346,65-76].

4. The control technique, which can be classified into open-loop and closed-loop control systems $[6-9,38,42,45-55,57-61,72,73,76-98]$

5. The current reference calculation, which uses the FFT algorithm and unity power factor algorithm $[6,43-46,54,61,71-73,87,92,99-102]$.

\subsubsection{Standard active filter circuits}

The current-fed converter filter (Fig. 2.11a) injects predefined current harmonics at PCC $[45,53-55,57-61,71,72,76,85,86,103]$. This configuration is only used in high power applications. The control strategy is very complex and sensitive to current variations in the DC-link inductor. The voltage-fed inverter (Fig. 2.11b) is simple to 
control and mostly used in low and medium power applications. The switching frequency in such systems is, however, high $[43,59,73,76,85-87]$.

(a)

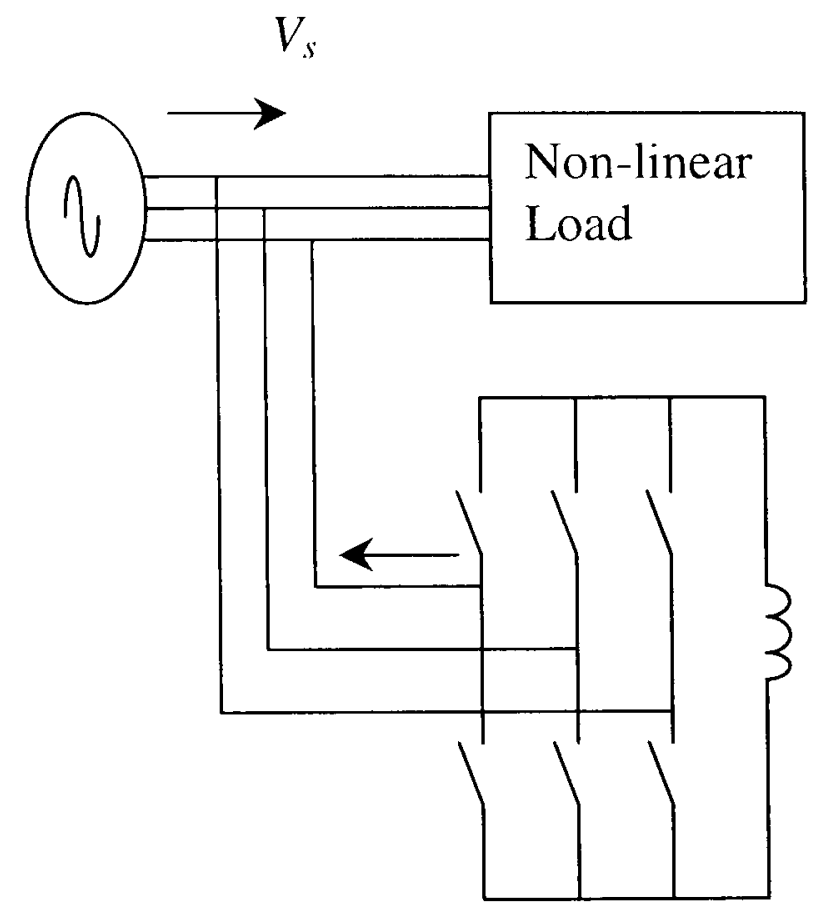

(b)

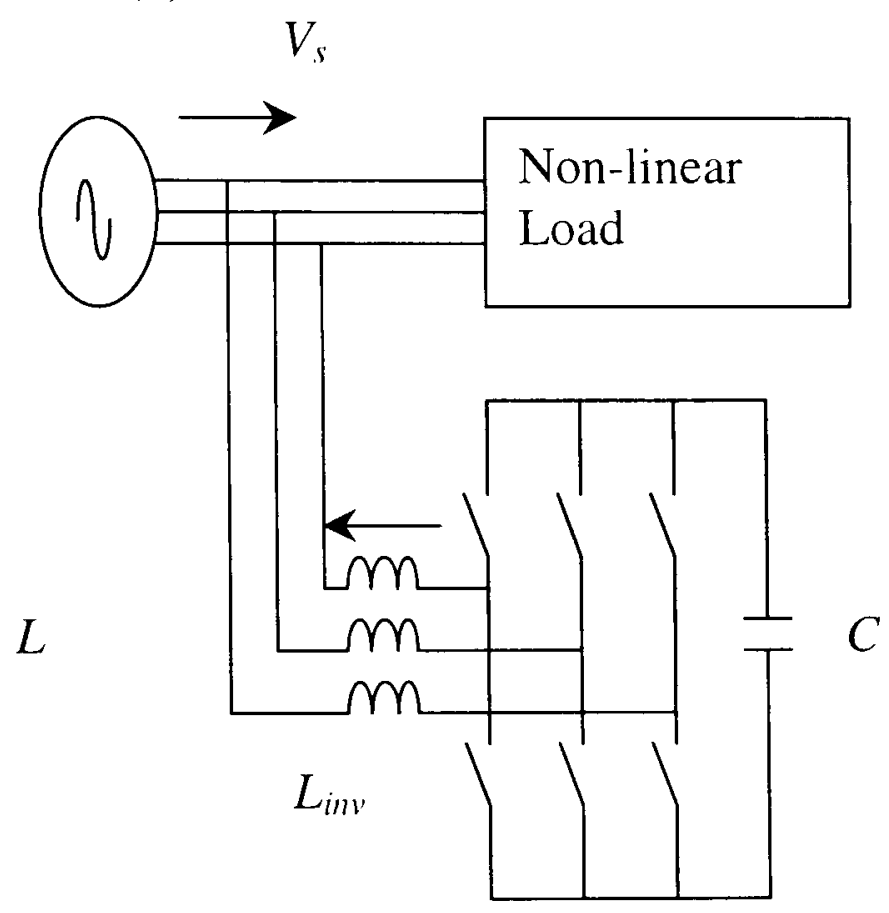

Fig. 2.11 Scheme of the Active Compensator Circuits
(a) Current-fed Inverter
(b) Voltage-fed Inverter

The configurations shown in Fig. 2.11 are a widely used types of filters. Three different configurations will be discussed further.

\subsubsection{Switched-capacitor active filter circuits}

This configuration (Fig. 2.12) was first developed by the power electronics group at Brunel University $[8,39,40]$. The circuit is very simple, using just one or two capacitors with very low values of current and limiting inductance. However, the control algorithm is very complicated compared to the inverter based filter configuration and is only applicable to single-phase systems or three-phase systems with three single-phase compensators. 


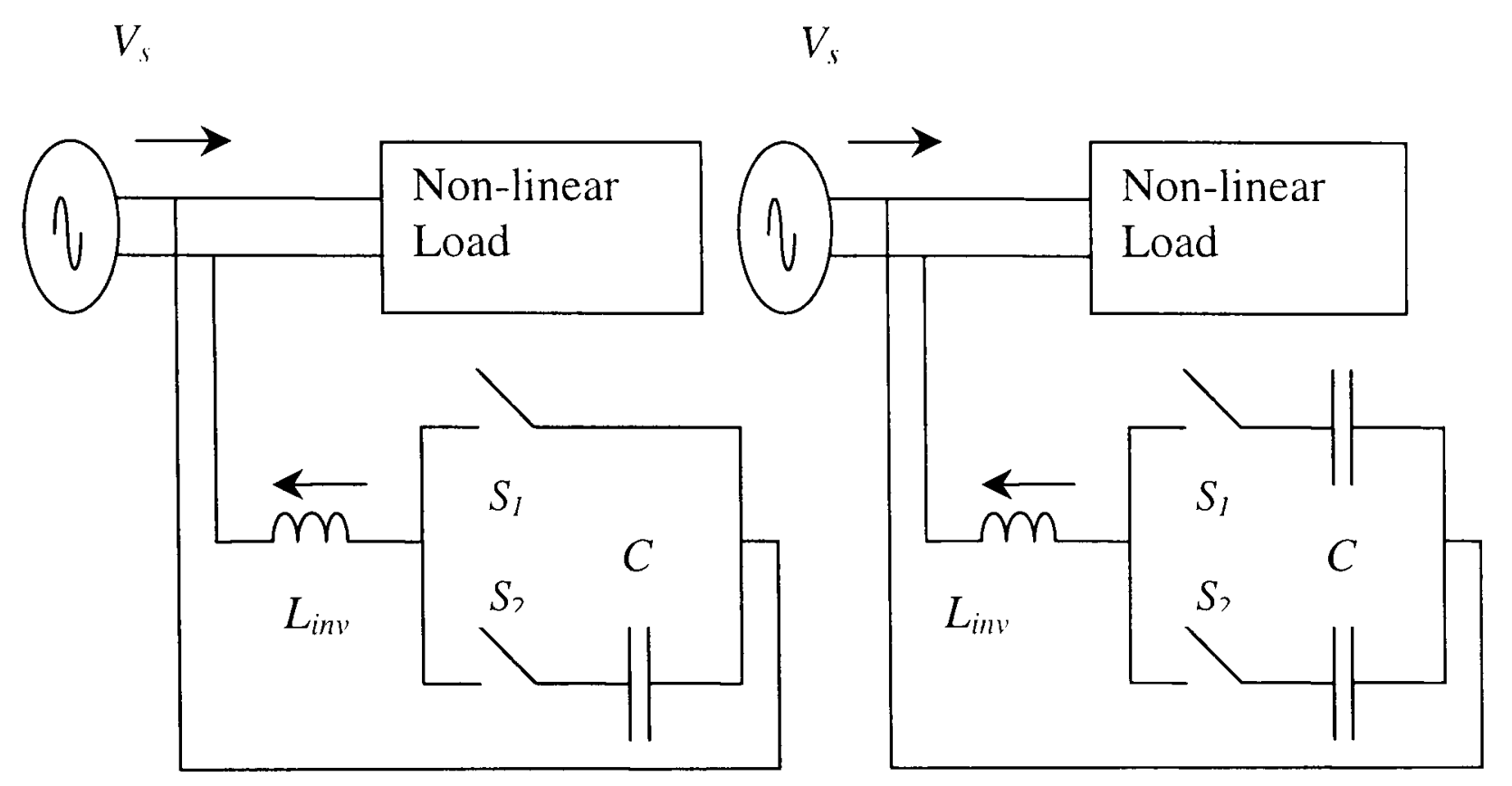

Fig. 2.12 Scheme of the Switched-Capacitor Filter

\subsubsection{Lattice structured active filter circuit}

Another type of active filter (Fig. 2.13), based on the lattice structures, has also been developed by the power electronics group at Brunel University. The control of these systems is time-consuming [48-51] and they are only used in single-phase systems, as with the previous one. Another drawback is that the circuit itself depends on the resonance during its operation.

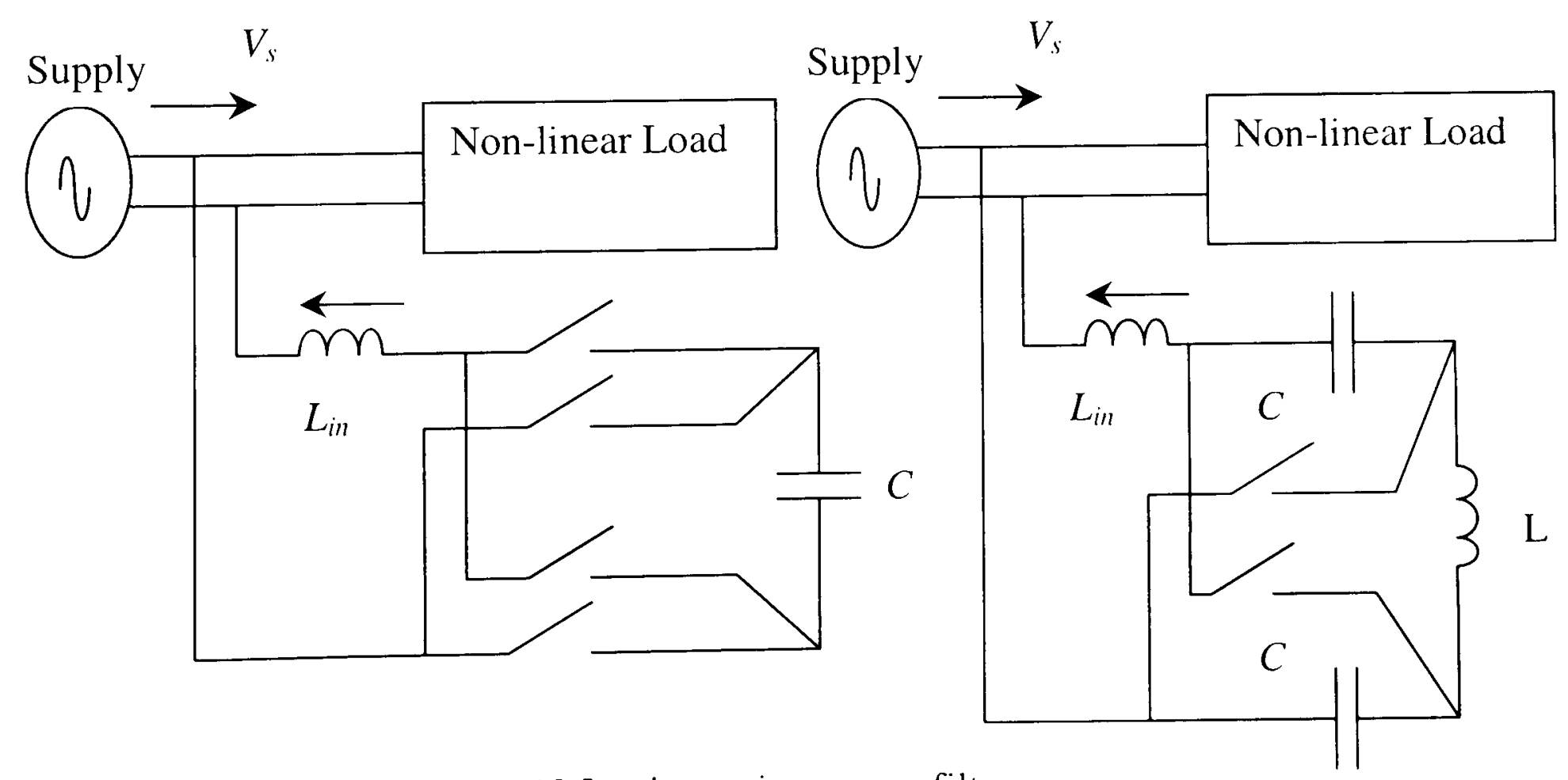

Fig.2.13 Lattice active power filter 


\subsubsection{Voltage controller active filter circuit}

Recently, the power electronics group at Brunel University has developed a new configuration of shunt active power filter as shown in Fig 2.14 [42]. It has several advantages over the other configurations:

1. It has low switching frequency

2. The components are of acceptable sizes

3. The reference signal is faster and better

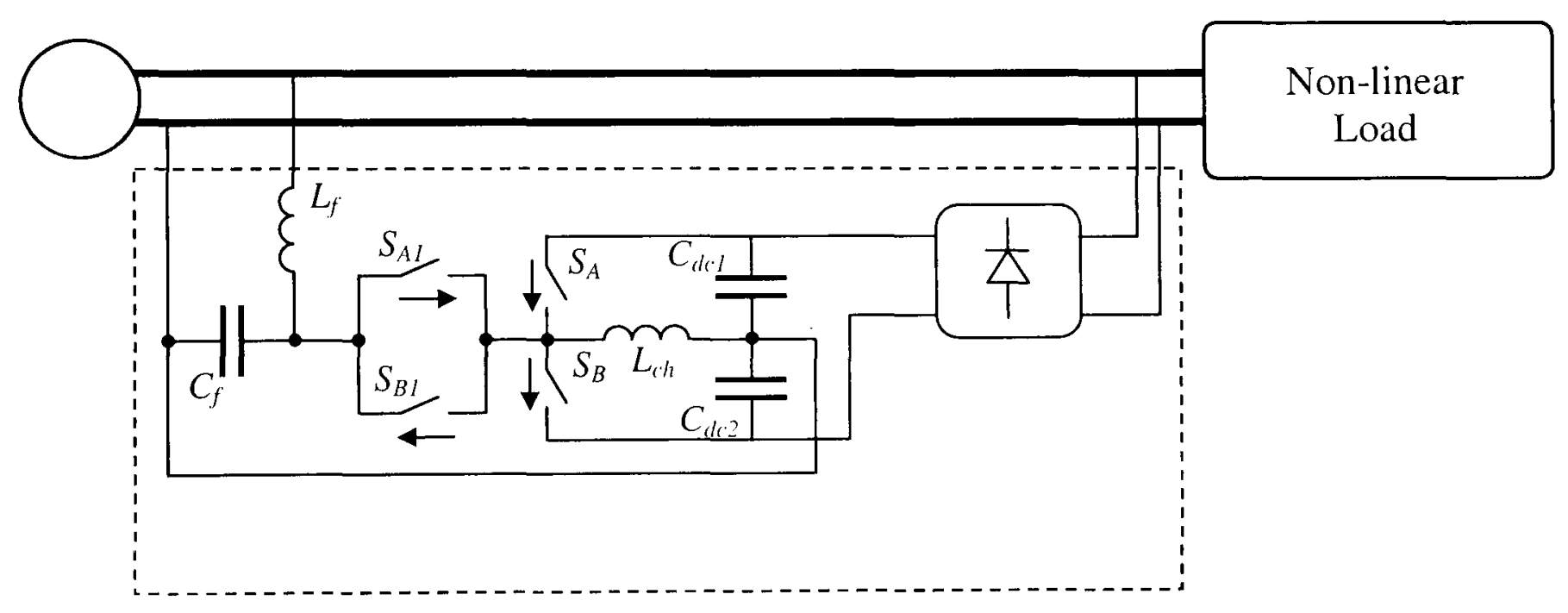

Fig. 2.14 Shunt Active Power Filter

However, this circuit configuration seems to have a complicated control algorithm due to the number of installed components, which may increase the cost of implementation.

\subsection{Series active filter}

This type of filter is not very common in industry (Fig. 2.15) [6.74,75,78,84,104]. The main drawback is that the filter has to withstand high rated load currents, which increases copper losses in the coupling transformer as well as the size of the filter. 
The main advantage is that it can be used to eliminate voltage waveform harmonics and to balance three phase voltages.

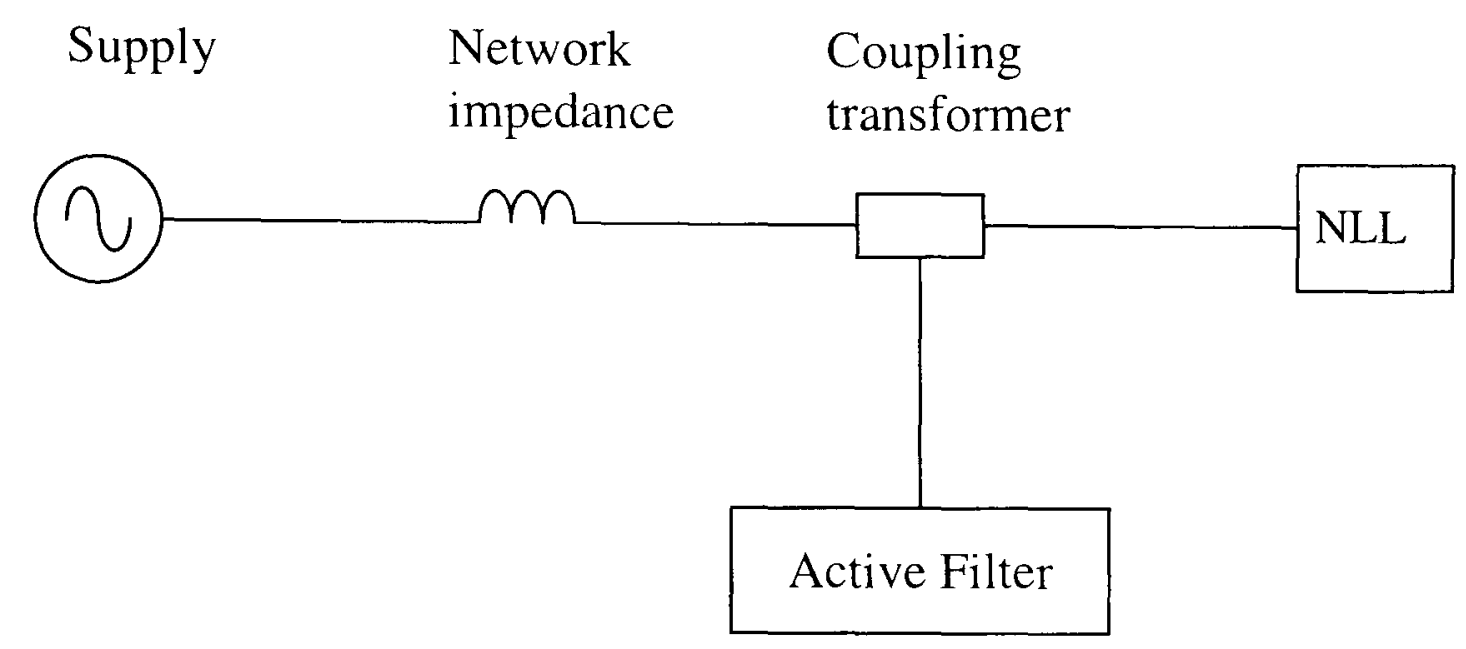

Fig. 2.15 Series active filter

\subsection{Reactive power optimisation in power systems}

Optimal power flow techniques, which have appeared in the literature, use a nonlinear approach based on Newton's formula [105-108]. The method works in large systems because of the supersparsity of the equations, with a large number of terms in the Hessian matrix, and sparsity techniques for adjusting control variables such as tap changing transformers and reactive power injections. These variables are changed based upon the sign of the associated dual variables to produce an acceptable solution. This requires a large CPU time to obtain an optimum solution. By breaking the problem down into active power and reactive power sub-problems, the classical optimal power was obtained [108]. Major drawbacks in Newton's method, which takes large steps at every iteration, have encouraged researchers to investigate the possibility of implementing gradient methods to solve the non-linear optimisation [109,110]. This method requires many inner loops to determine the optimal step size of the variables. Some researchers [24.111.112] have considered the application of non-linear formulae in solving the power dispatch. such as Volt/VAR control on 
radial distribution systems, using decomposition techniques which also had convergence problems. The method is, however, efficient for the planning of a distribution system. This study produces the final solution and the voltage profile of the system. The authors [113-116] use a non-linear objective function to reflect system losses. The control variables are the generator terminal voltages and transformer tap ratios. Newton's method is used again for the dispatch. The method does not incorporate a step size in the procedure.

In order to obtain satisfactory results within a reasonable CPU time, many methods have been tried to linearise the system of equations describing the relationships between controlled, controllable variables and loss sensitivity indices [117-119]. However, in large-scale systems, the incorporation of integer variables would require a large memory space.

Another application of using linear programming to reactive power control calculations has been presented in [120-122]. This method incorporates the usual reactive power control devices and was particularly successful in rescheduling tapchanging transformers. Solutions were constrained within limits set by bus voltages and network reactive power flows.

To minimise the real power losses, an improved linear sensitivity matrix for VARS and volts was presented in [123-125]. In [126,127] P-optimisation and Qoptimisation methods for optimal real and reactive power dispatch in the economical opcration of power systems was presented. The method was very time consuming because of a large number of calculations of Jacobian and sensitivity matrices. 
Different programming methods were presented for optimising the reactive power to find the optimal voltage profile, which minimised power losses and satisfied certain constraints [128].

In [129-131], the decomposition technique, in which the primary and dual solutions of the revised simplex method are utilised, reduced the required CPU time and memory space. The available algorithms implemented different mathematical programming methods for optimising the VAR [132-136]. The proposed methods treated normal operation and did not consider contingency cases. The system operation characteristics of new reactive power sources in [137-139] treated all variables as real, and integer variables have not been considered in the optimisation.

Using most of the available methods, the operation sub-problem suffers from a long CPU solution time and large memory requirements, which means it cannot be implemented on large-scale systems. For the investment sub-problem, most of the methods presented have similar formulations except that the set of candidates (buses) on which the new reactive power sources would be imposed are different. Generally, proposed studies for the solution of the reactive power allocation problem have suffered from numerous drawbacks, and intensive work is still being done on developing algorithms that can be applied to large-scale systems.

\subsection{Expert systems, fuzzy logic and neural network applications in}

\section{power systems}

The papers [140-143] present an artificial intelligence approach to the optimal ractive power control problem. The artificial neural network enhanced by fuzzy sets 
is used to determine the memberships of control variables corresponding to the given load values. In addition, a mathematical formulation for the reactive optimal power control problem using fuzzy set theory was presented. In [144] an interactive fuzzy method for optimal multi-objective VAR planning in power systems was proposed, taking into account economical operating conditions, the system security margin and voltage deviation. Paper [145] described a new approach to the design of a controller for a static fuzzy logic-based VAR stabiliser for power system control. Artificial intelligence (AI) tools such as expert systems, fuzzy logic and neural network applications in power electronics and motion control were presented in [146]. Theoretical principles of each system were given in a simple manner. In the later papers [147-149] harmonic source monitoring and identification using neural networks to estimate harmonic components in a power system, and a neural-network based method for predicting power system voltage harmonics were presented. Future control strategies for active filters can be seen in Fig. 2.16

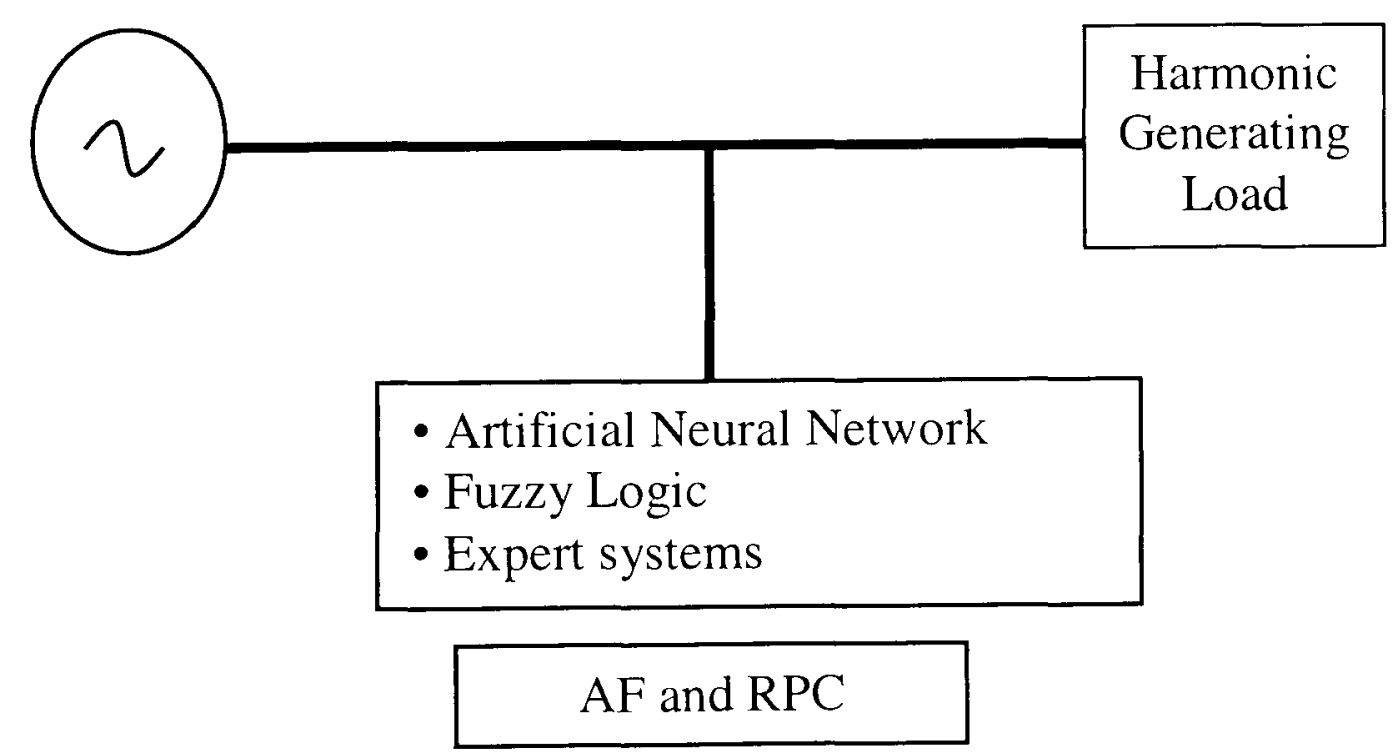

Fig. 2.16 Future control strategies for active filters 


\subsection{Summary}

This chapter has described the state of the art for reactive power compensation and harmonics analysis, as presented in the literature and papers surveyed. From that point, the control strategy and performance of the proposed power circuit will be discussed in the following two chapters. 


\section{Chapter 3}

\section{A New Method for Power Quality Improvement}

\subsection{Introduction}

Power quality problems may originate from the network or from the consumer. The permissible limits for these disturbances are specified by standards and regulations. The basic requirements of the supply system are:

- Power transmission must be economical.

- Power system failure must be low.

- The quality of the power supply must be high.

Power factor improvement in power systems with non-sinusoidal supply voltage has been a subject of concern for a long time. As a consequence, various types of compensators have been developed to improve the efficiency of power transmission and distribution by increasing the power factor. At present many methods exist to eliminate or reduce the effects of harmonics on power systems $[6,10]$.

Today, switching devices are increasingly used in the electric power system to increase productivity. On the other hand, these devices produce harmonics, voltage distortion and may cause reduction in power factor.

The previous chapter discussed extensive research related to power factor correction, harmonic elimination and filter design $[8,9,38-40,42,48-52]$. Today, filter circuits. dynamic compensation and conventional capacitors are in use, although with some 
problems. In this chapter, a new control method is proposed which eliminates harmonics and improves power factor; its control strategy is also described. The principles of design, analysis and calculation of the new IGBT-equipped are presented, and it is shown that this circuit is able to compensate rapidly for changing loads and reactive power. A single-phase control concept is discussed for simplicity, although the results can be implemented in three-phase systems without any problems.

The proposed compensator for reactive power and harmonic compensation can be applied to medium and low voltage distribution systems. Design and analysis of the circuit is described.

The block diagram, shown in Fig 3.1, represents the proposed active power filter combination. The block diagram shows ten main sections constituting the power active filter structure. The harmonic current generated by the non-linear load is detected and fed back to the reference current estimator, in conjunction with the other system variables. The resulting reference signal drives the overall system controller. which, in turn, generates the control output necessary for the switching pattern. The resulting filter current is also detected and fed back to the controller. The power and filter circuits are then connected to the PCC.

\subsection{Proposed Circuit and its Control Strategy}

Fig. 3.2 shows the proposed active filter and circuit representation with the current controller. A non-linear load draws a load current from the network. The 
compensator is connected in parallel with the non-linear load and provides the compensator current.

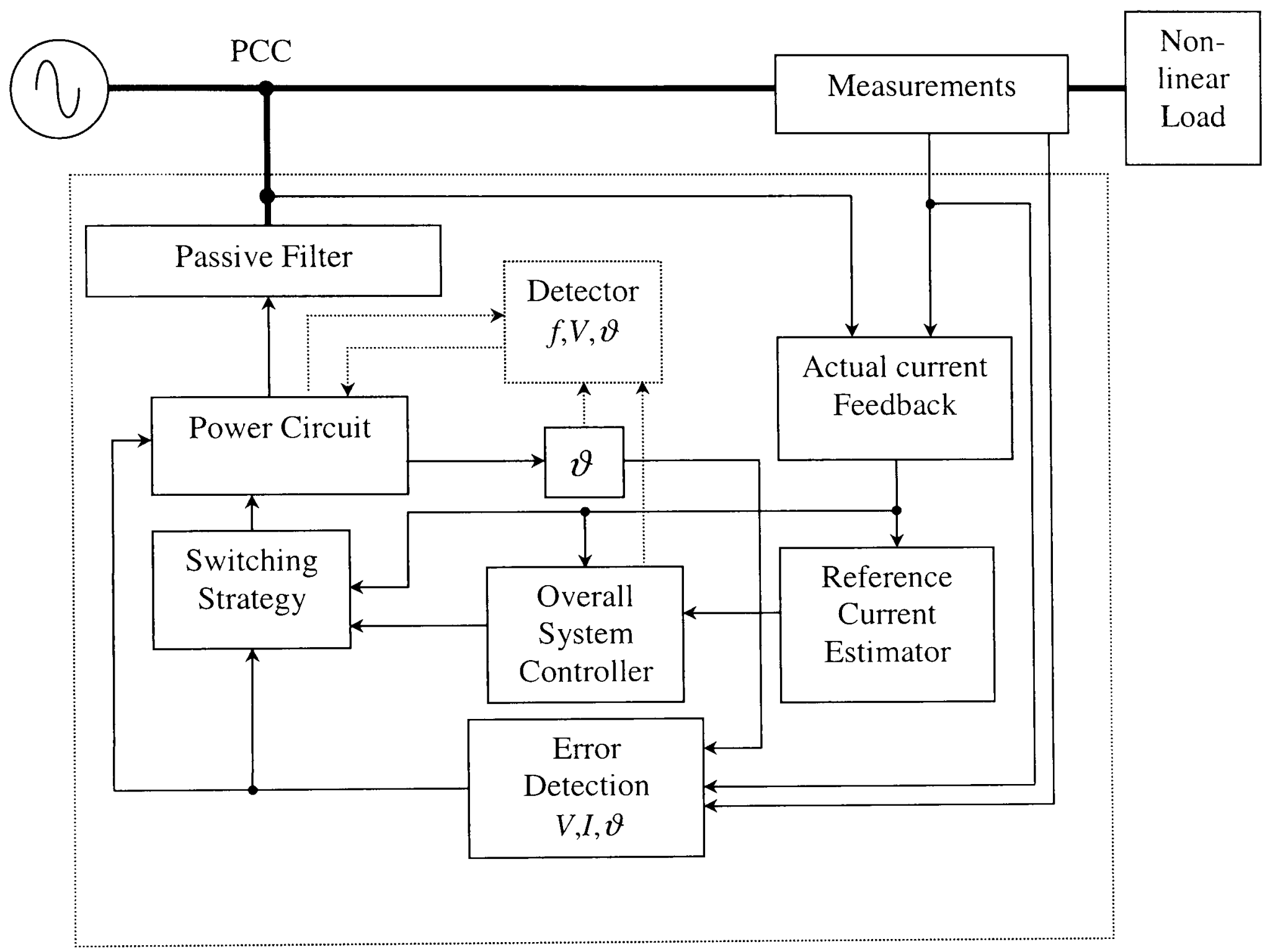

Fig.3.1: Proposed complete block diagram for shunt active power filters

An energy storage capacitor and voltage source inverter is connected to an LCL-TNetwork-AC filter to supply a controlled inverter current $i_{i n v}$ which is controlled by a hysterisis control system. This requires a variable hysterisis width, which depends on the filter inductance, $L_{i n v}$, the inverter DC voltage, $V_{D C}$, the desired switching frequency, $f_{S}$, and the filter capacitance, $C_{D C}$. The power switches of the inverter consist of IGBTs and diodes. At a given node (PCC), the supply system and nonlinear load (NLL) consists of various voltage sources, current sources, network impedances and load impedances, which are connected to other node. 


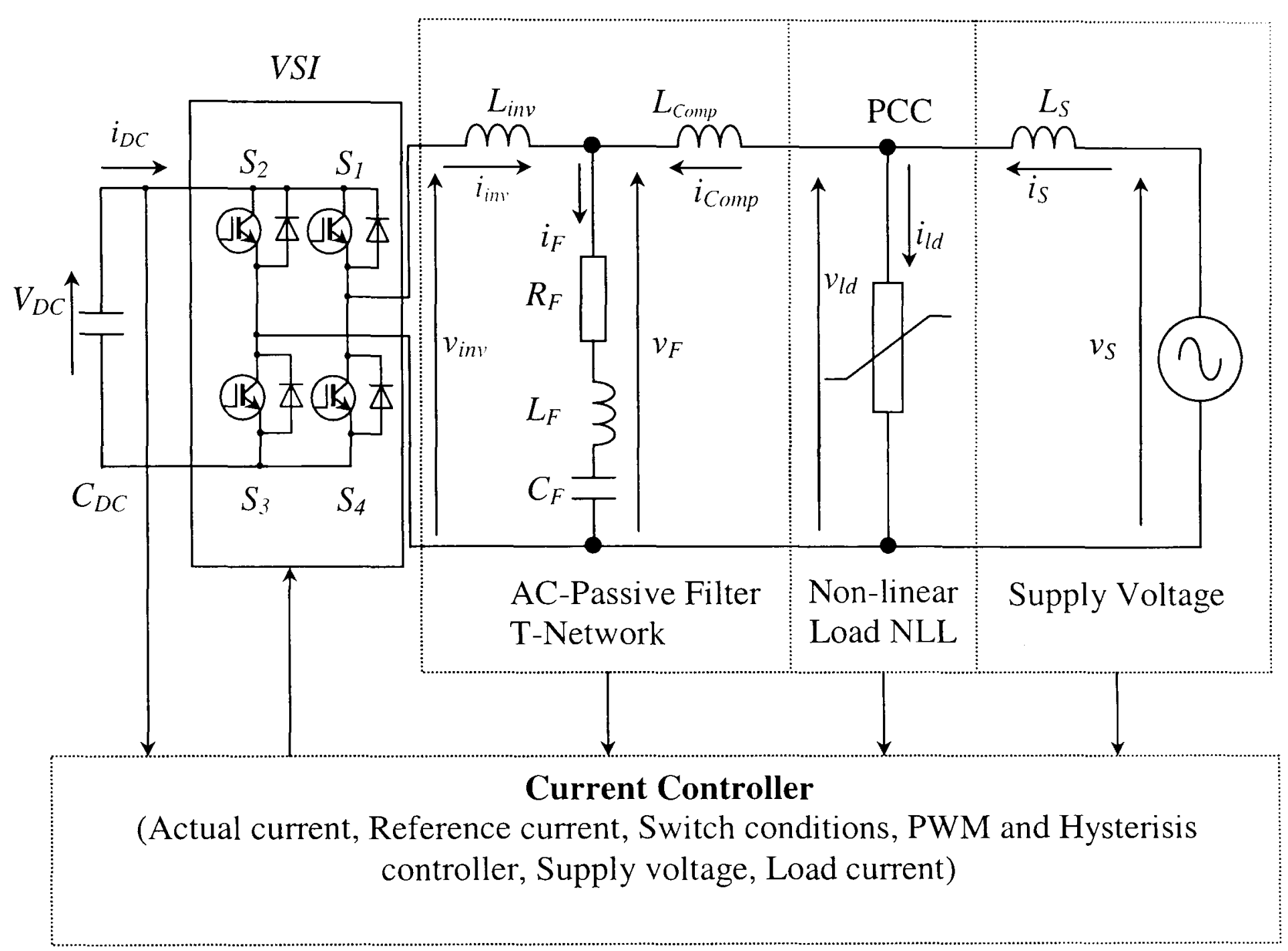

Fig.3.2: Single-Phase Full Bridge Inverter-Filter Scheme

Knowing the load current harmonic-content, $\underline{I}_{h}$, and network harmonic impedance,

$\underline{Z}_{h}$, the voltage distortion can be calculated as

$V_{h i}=Z_{h_{i}} \cdot I_{h_{i}}$

(where $i=1,2,3, . . N_{\text {Harmomics }}$ )

The network impedance $\underline{Z}_{h}$ is expressed for each individual harmonic, $i$, as

$Z_{h_{i}}=\left(\frac{Z_{F_{i}} \cdot Z_{S_{i}}}{Z_{F_{i}}+Z_{S_{i}}}\right)$

where, $\underline{Z}_{F}$ and $\underline{Z}_{S}$ are the vectors of filter and supply impedance at each harmonic, $i$.

respectively. 
A voltage source inverter (VSI) is used to eliminate the harmonics and improve the power factor in the power system at the PCC. At the output of the VSI, an inductance $L_{i n v}$ is connected to reduce or eliminate the harmonics in the output inverter current, $i_{i n v}$, which would reflect on the supply voltage waveform as a superimposed high frequency component. A passive filter is also included to smooth any remaining ripple in the inverter current and to reduce the power needed by the inverter to fulfil its designed task. The filter contains a filter resistance, $R_{F}$, a filter capacitor, $C_{F}$, and a filter inductance, $L_{F}$. The equivalent internal impedance of the main power system is given by the inductance, $L_{S}$, (neglecting resistance). The VSI consists of two sets of IGBT switching devices connected in parallel (H-Bridge inverter) (Fig. 3.2). Each IGBT is connected to a free-wheeling diode in parallel. The two switches (top and bottom) are operated in anti-phase so that the inverter current $i_{i n v}$ flows through the two branches alternately.

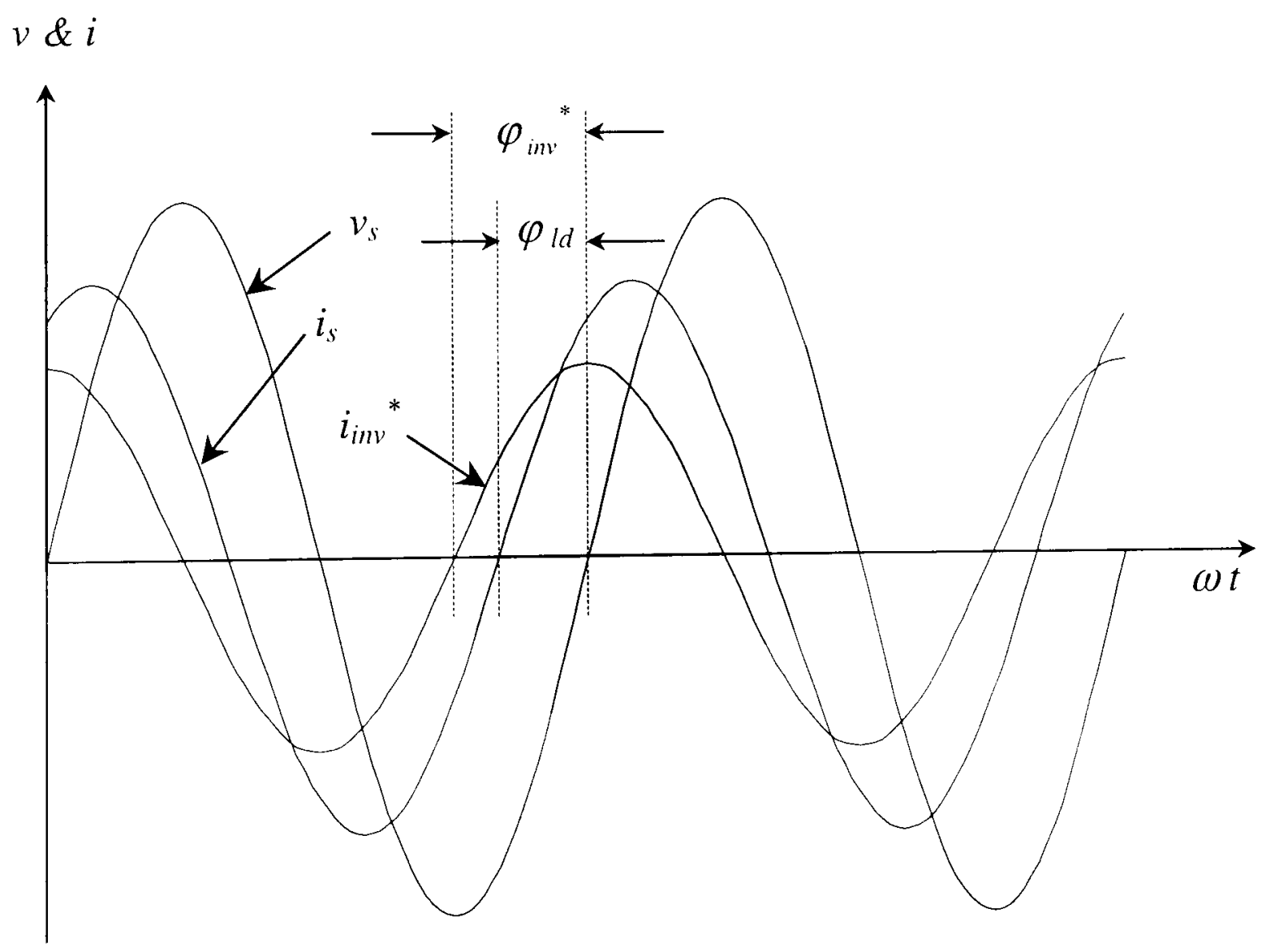

Fig.3.3: Magnitude and phase change between inverter output current $i_{i m}$ and main supply current $i$, 
Magnitude and phase changes between the desired inverter output current, $i_{i n}{ }^{*}$. and the main network current, $i_{s}$, is given in Fig.3.3:

$$
i_{i n v}{ }^{*}=\sum_{i=1}^{N_{\text {harmonics }}} \hat{I}_{i n v i}{ }^{*} \cdot \sin \left(i \omega_{1} t-\varphi_{i n v i}{ }^{*}\right)
$$

The actual supply current value, $i_{S}$, is measured with a constant sampling period, $T_{S}$. If the current reaches one of the limits, $i_{t o l}$, then inverter switching is required after the delay time $T_{D}$ (Fig.3.4). Normally, the mains network acts as an ohmic-inductive consumer and the computation can be performed with a given supply power $P_{s}$ and a power factor, $\cos \varphi_{l d}$. The peak value of the sinusoidal desired inverter current can then be calculated as in the following lines.

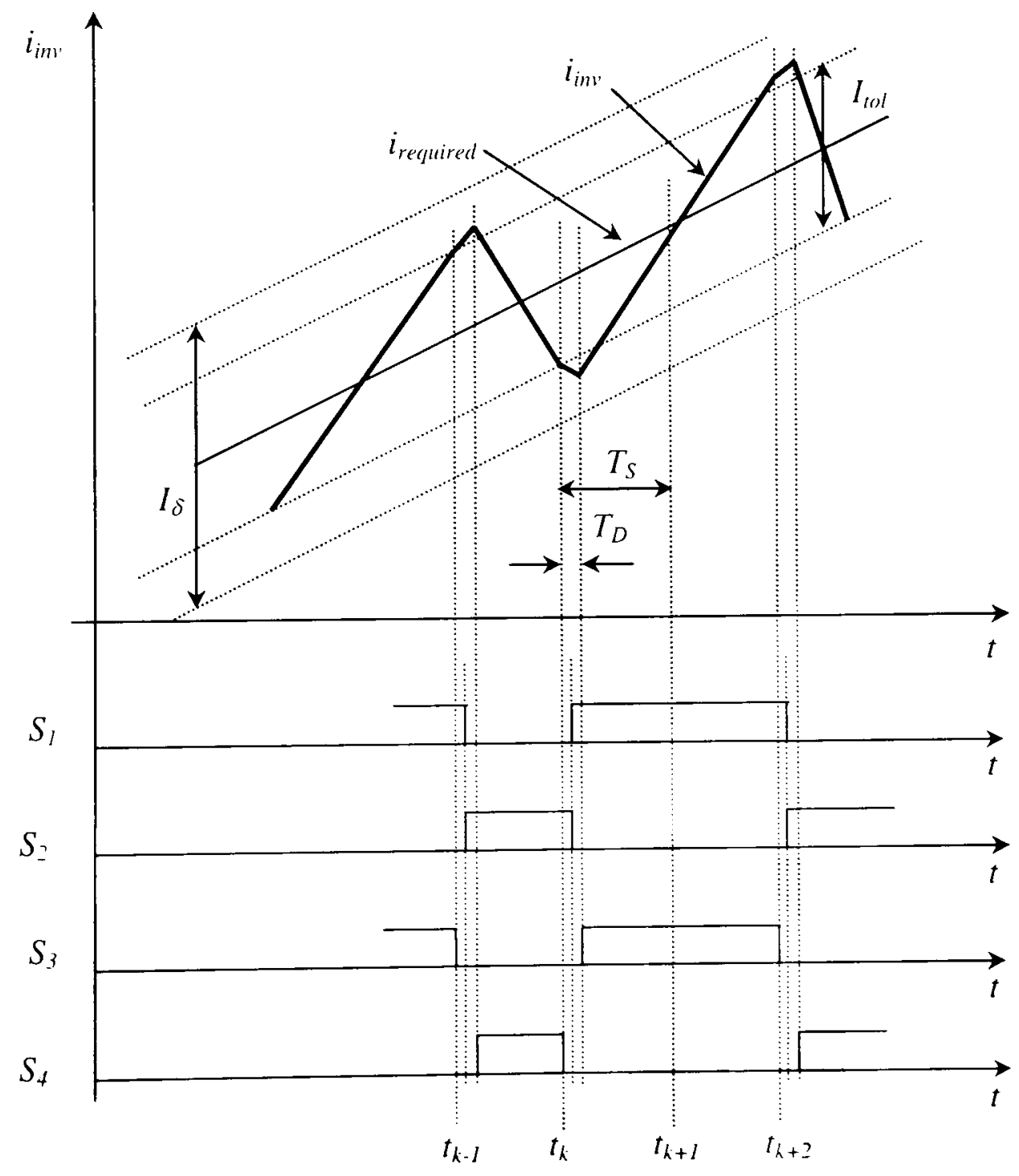

Fig.3.4: PWM-hysterisis switching technique 
The current controlled by the inverter in the proposed circuit is the inverter current $i_{i n v}$. The inverter in this case acts as a current source pumping $i_{i m v}$ through $L_{i m v}$ and into the rest of the network. Since the impedance of the nonlinear load is much higher than the supply impedance, the load will not draw any of the inverter-injected current and hence the circuit reduces to the inverter current being forced back into the supply. The circuit reduces to two branches, namely the passive-filter branch and the supplyimpedance. The transfer ratio, $m_{L C L}$, for the inverter current to the supply side can be approximated by the following equation

$$
m_{L C L} \approx\left|\frac{Z_{F}}{Z_{F}+\left(Z_{S}+Z_{\text {Comp }}\right)}\right|
$$

which can be simplified to the following equation for the individual harmonic $i$

$$
m_{L C L_{i}} \approx \sqrt{\frac{R_{F}^{2}+\left(i \omega_{1} L_{F}-\frac{1}{i \omega_{1} C_{F}}\right)^{2}}{R_{F}{ }^{2}+\left(i \omega_{1}\left(L_{F}+L_{S}+L_{C o m p}\right)-\frac{1}{i \omega_{1} C_{F}}\right)^{2}}}
$$

The value of $m_{\mathrm{LCl}_{i}}$ can be seen as a correction factor for the reference inverter current at each harmonic $i$.

The phase angle correction for the harmonic $i$ of the inverter current, $\varphi_{i m i}$, is defined as:

$$
\varphi_{i m i_{i}}^{*}=\varphi_{I d h_{i}}-\left[\tan ^{-1}\left(\frac{\left(i \omega_{1} L_{F}-\frac{1}{i \omega_{1} C_{F}}\right)}{R_{F}}\right)-\tan ^{-1}\left(\frac{i \omega_{1}\left(L_{F}+L_{S}+L_{C o m p}\right)-\frac{1}{i \omega_{1} C_{F}}}{R_{F}}\right)\right]
$$

where, the value of $\varphi_{l d h_{i}}$ is the load phase angle at the harmonic $i$. 
The peak value of this inverter current reference can now be calculated for the harmonic component $i$, as being

$$
\hat{I}_{i m v^{\prime} i}^{*}=\frac{\sqrt{2} \cdot I_{l d h_{i}}}{m_{L C L i}}
$$

The individual harmonic components of the load current, $I_{l_{h_{h}}}$, is calculated by applying the Fourier analysis to the load current $I_{l d}$. The rms value of the fundamental component of the load current $I_{l d}$ can be calculated from the following equation.

$$
I_{l d 1}=\frac{P_{l d}}{\left[V_{S} \cdot\left(\cos \left(\varphi_{l d}\right)\right) \cdot m_{L C L}\right]}
$$

The values of the inductors, the pattern of the switching functions and the filter characteristics define the network current. Basically the PWM pattern can be used for the control of the inverter so as to generate a desired output current waveform. According to the simulation results presented later on in this thesis, the hysterisis control method seems to be a more suitable solution because it guarantees stable operation. The degree of compliance to the EMC regulations as will be discussed in chapter 6 , will favour the introduction of a PWM technique to modify the hysterisis controller. The hysterisis technique with PWM is discussed in the following sections and the performance analysis with the ordinary hysterisis control is presented later on in the simulation and in the practical results.

Fig.3.4 shows the tracking strategy for the PWM modified hysterisis control. The controlled current is sampled with a small tolerance bandwidth and is evaluated in the 
control unit. Referring to Fig.3.4, to achieve a hysterisis control band of width $I_{\delta}$, the tolerance current $I_{t o l}$, which is controlled, can be calculated from:

$$
I_{t o l}=I_{\delta} \cdot \frac{T_{S} / 2}{\left(T_{S}+T_{D}\right)}
$$

The switching modes of the inverter switches, shown in Fig.3.5, are given in Table.3.1. Direct switching from state I to state III must be avoided, in order to reduce the high rates of current change and the consequent necessity of higher switching frequency. Alternatively, Mode I must be followed only by mode II. If it is necessary to transfer to mode III, then mode IV must precede it first in order to ensure that the switching losses are reduced to minimum. In other words, the zero switching state for mode I (mode II) or the one for mode III (mode IV) is always to precede and succeed its active switching state. The four permissible switching states for switches $S_{1}, S_{2}, S_{3}$ and $S_{4}$ are presented in the following table

Table.3.1: Switching pattern of the inverter

\begin{tabular}{|c|c|c|c|c|c|}
\hline mode & $\boldsymbol{S}_{\boldsymbol{I}}$ & $\boldsymbol{S}_{2}$ & $\boldsymbol{S}_{3}$ & $\boldsymbol{S}_{\boldsymbol{t}}$ & $\boldsymbol{V}_{\text {inv }}(\boldsymbol{t})$ \\
\hline I & 1 & 0 & 1 & 0 & $+V_{D C}$ \\
\hline II & 1 & 0 & 0 & 0 & 0 \\
\hline III & 0 & 1 & 0 & 1 & $-V_{D C}$ \\
\hline IV & 0 & 1 & 0 & 0 & 0 \\
\hline
\end{tabular}

The current paths for each of the four modes of operation are shown in Fig. 3.5 with thick lines. 


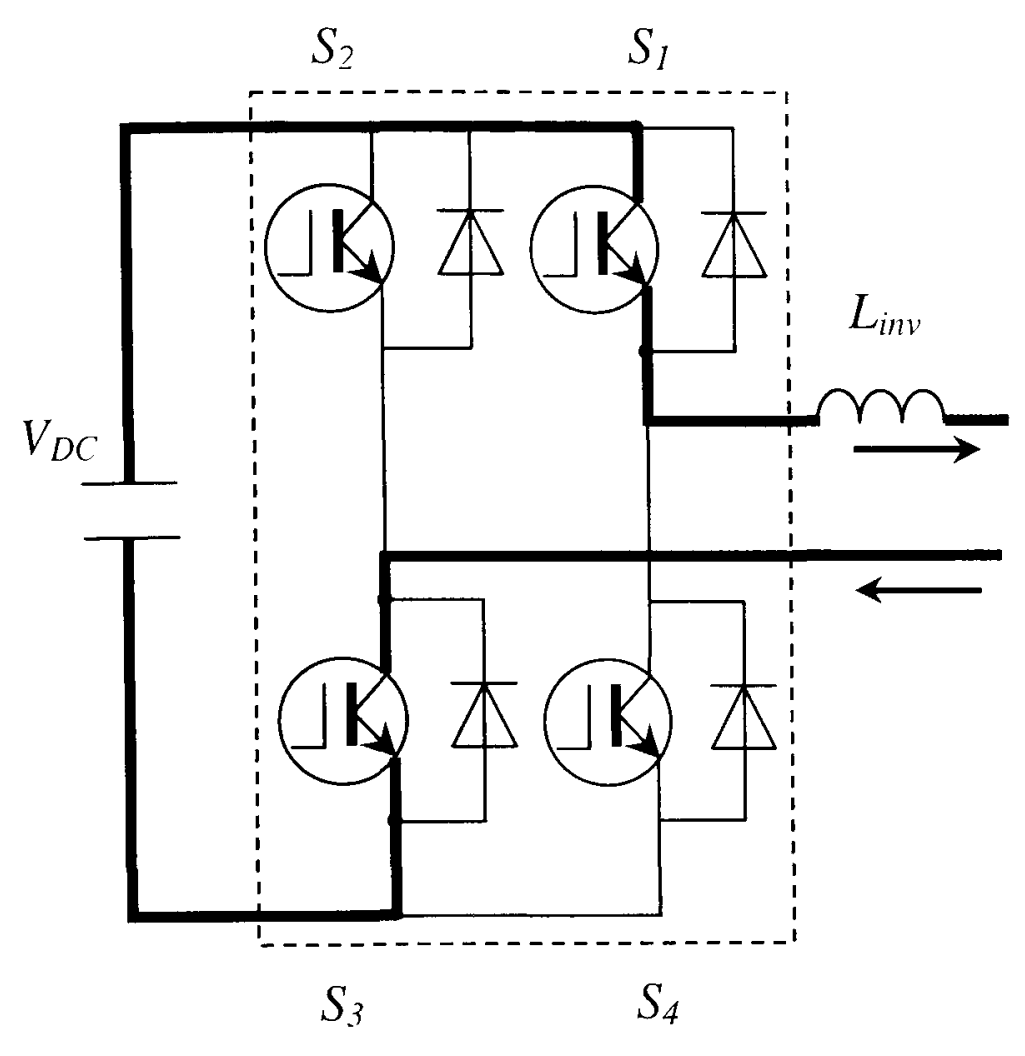

(a) Mode I

Positive output voltage

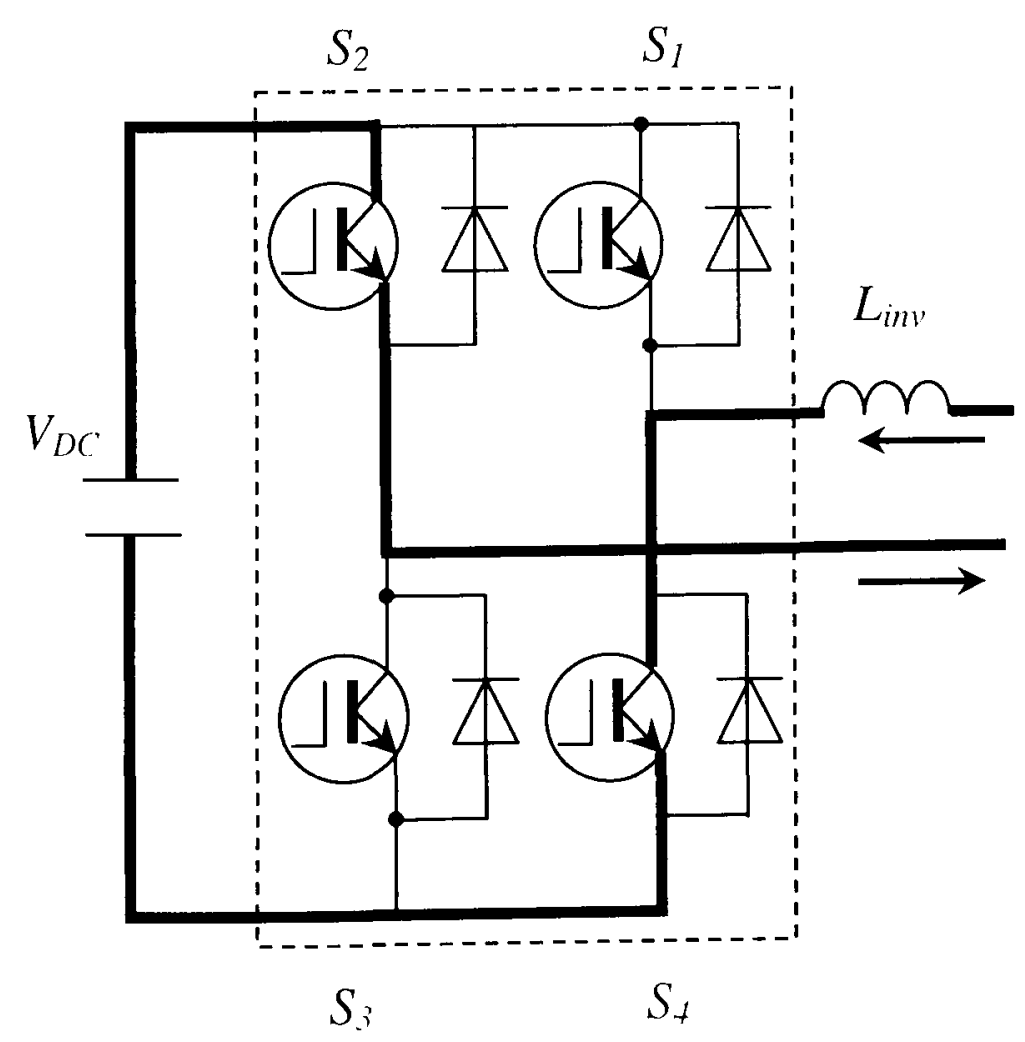

(c) Mode III

Negative output voltage

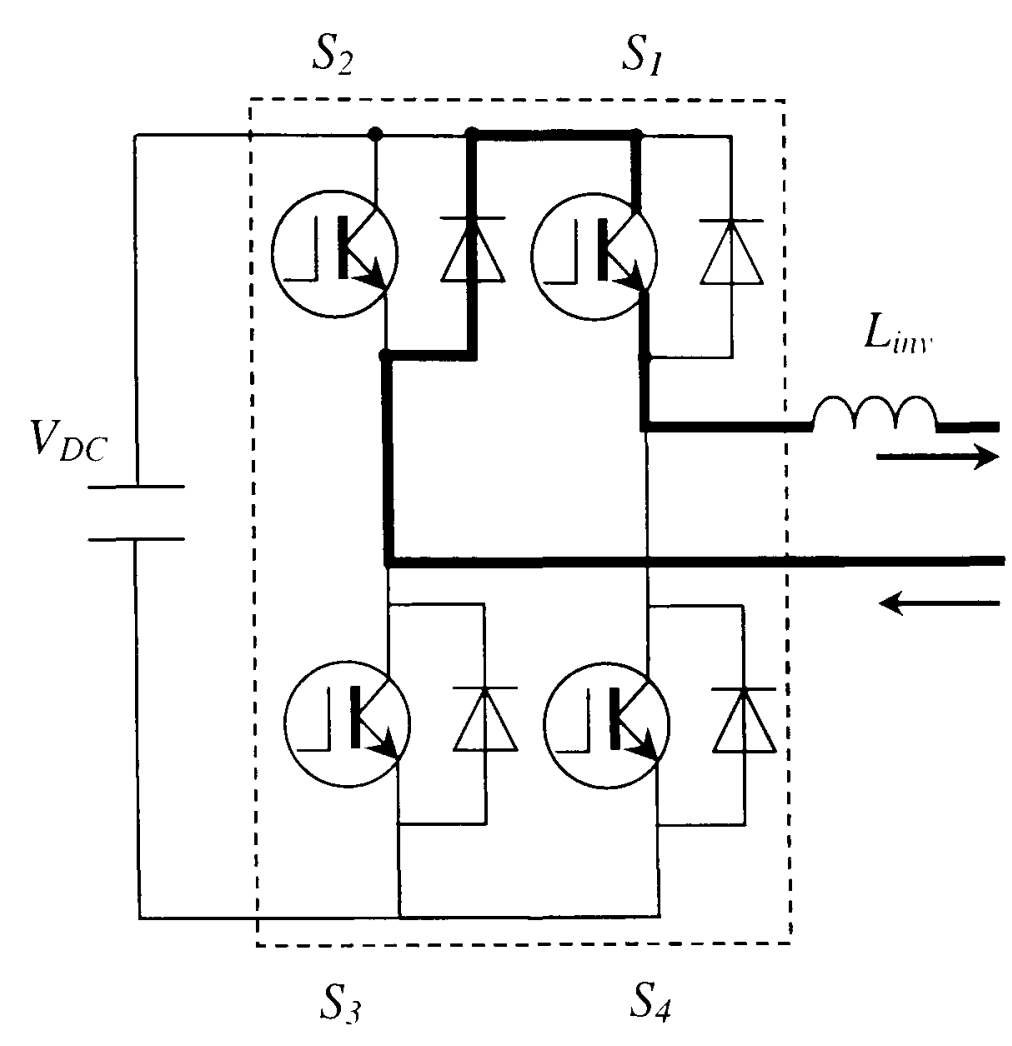

(b) Mode II

Zero output voltage

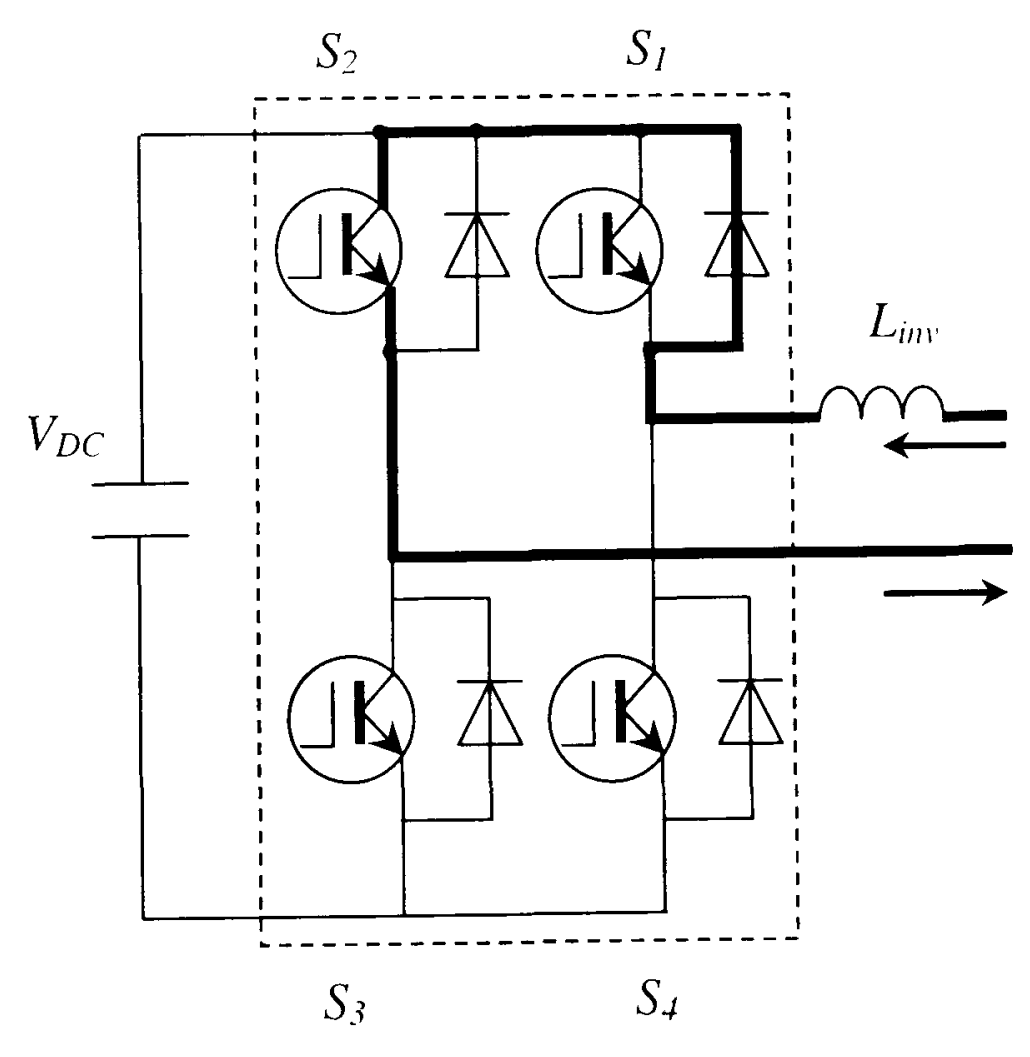

(d) Mode IV

Zero output Voltage

Fig.3.5: Modes of operation of the inverter circuit 
To smooth high frequency harmonics in the mains supply current and supply voltage. a passive filter is designed and connected to the VSI. The filter resistance, $R_{F}$, is inserted to restrain the oscillations of the network capacitor and inverter inductance. A lower value of $R_{F}$ gives the best smoothing of the network current and supply voltage. On the other hand, the filter capacitor $C_{F}$ must be large enough to absorb the current and voltage ripples.

In order to ensure the circuit operation, the following inequalities must be satisfied:

- First of all it has to be ensured that the overall sampling and delay periods are smaller than the time taken by the actual controlled inverter current, $i_{i m}$, to cross half the boundary $I_{\delta}$. This can be formulated as:

$$
\frac{I_{\delta} \cdot\left(L_{S}+L_{C o m p}+L_{i n v}\right)}{2 \cdot V_{D C}} \geq\left(T_{S}+T_{D}\right)
$$

- The resonance frequency $f_{r}$ of the circuit diagram of Fig.3.2 is:

$$
f_{r} \leq \frac{1}{2 \pi} \cdot \sqrt{\frac{1}{\left(L_{F}+\frac{\left(L_{S}+L_{C o m p}\right) \cdot L_{i m v}}{L_{S}+L_{C o m p}+L_{i n v}}\right) \cdot C_{F}}}
$$

- To avoid the resonance frequency, the following assumption can be made with sampling frequency $f_{S}$ :

$$
f_{S} \geq 20 \cdot f_{r}
$$

- The filter capacitor $C_{F}$ can be calculated by substituting equation (3.11) into (3.12) and rearranging the resulting equations.

$$
C_{F} \approx 10 \cdot \frac{1}{\left(L_{1}+\frac{\left(L_{s}+L_{C o m p}\right) \cdot L_{i m v}}{L_{S}+L_{C o m p}+L_{i n v}}\right)} \cdot T_{S}^{2}
$$


- Then, the filter resistance $R_{F}$ can be expressed as in the following imperical equation

$$
R_{F} \approx 0.15 \cdot \sqrt{\frac{1}{C_{F} \cdot\left(L_{F}+\frac{\left(L_{S}+L_{C o m p}\right) \cdot L_{i n v}}{L_{S}+L_{C o m p}+L_{i n v}}\right)}}
$$

- The desired current control occurs in a time interval, $T_{s}$, with a constant length given by:

$$
T_{S} \leq \frac{2 \pi}{20} \cdot \sqrt{\left(L_{F}+\frac{\left(L_{S}+L_{C o m p}\right) \cdot L_{i n v}}{L_{S}+L_{C o m p}+L_{i n v}}\right) \cdot C_{F}}
$$

- The controller delay time $T_{D}$ of the switching circuit can be expressed by substituting the value of $T_{s}$ into equation (3.10) and rearranging the terms of the resulting equation

$$
T_{D} \leq \frac{I_{\delta} \cdot\left(L_{S}+L_{C o m p}+L_{i m}\right)}{2 \cdot V_{D C}}-T_{S}
$$

A detailed system design combining both the inverter and the filter component value computation is presented in the block diagram of Fig.3.6. 


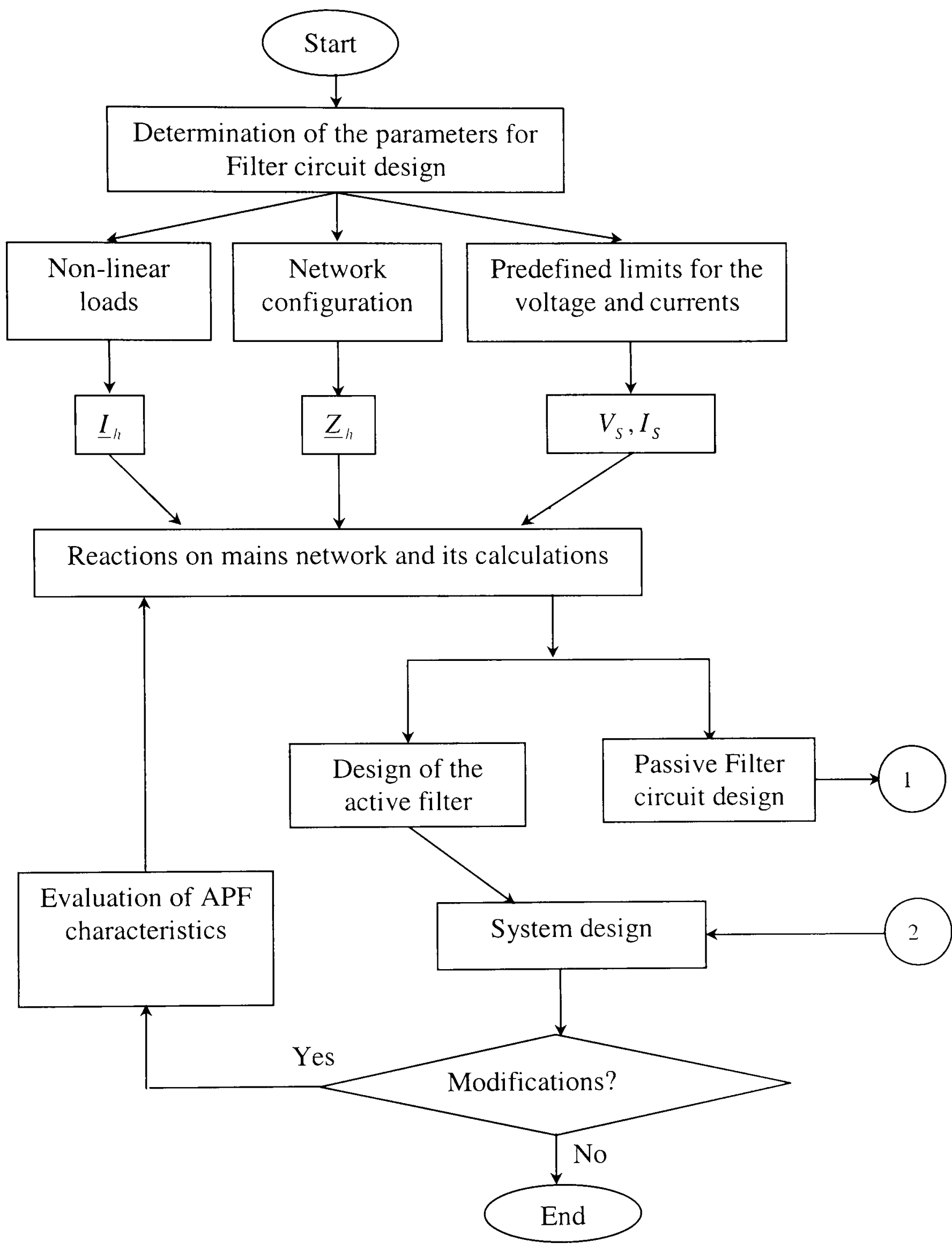

Fig.3.6: Flow chart for the determination of harmonic evaluation and filter circuit design 


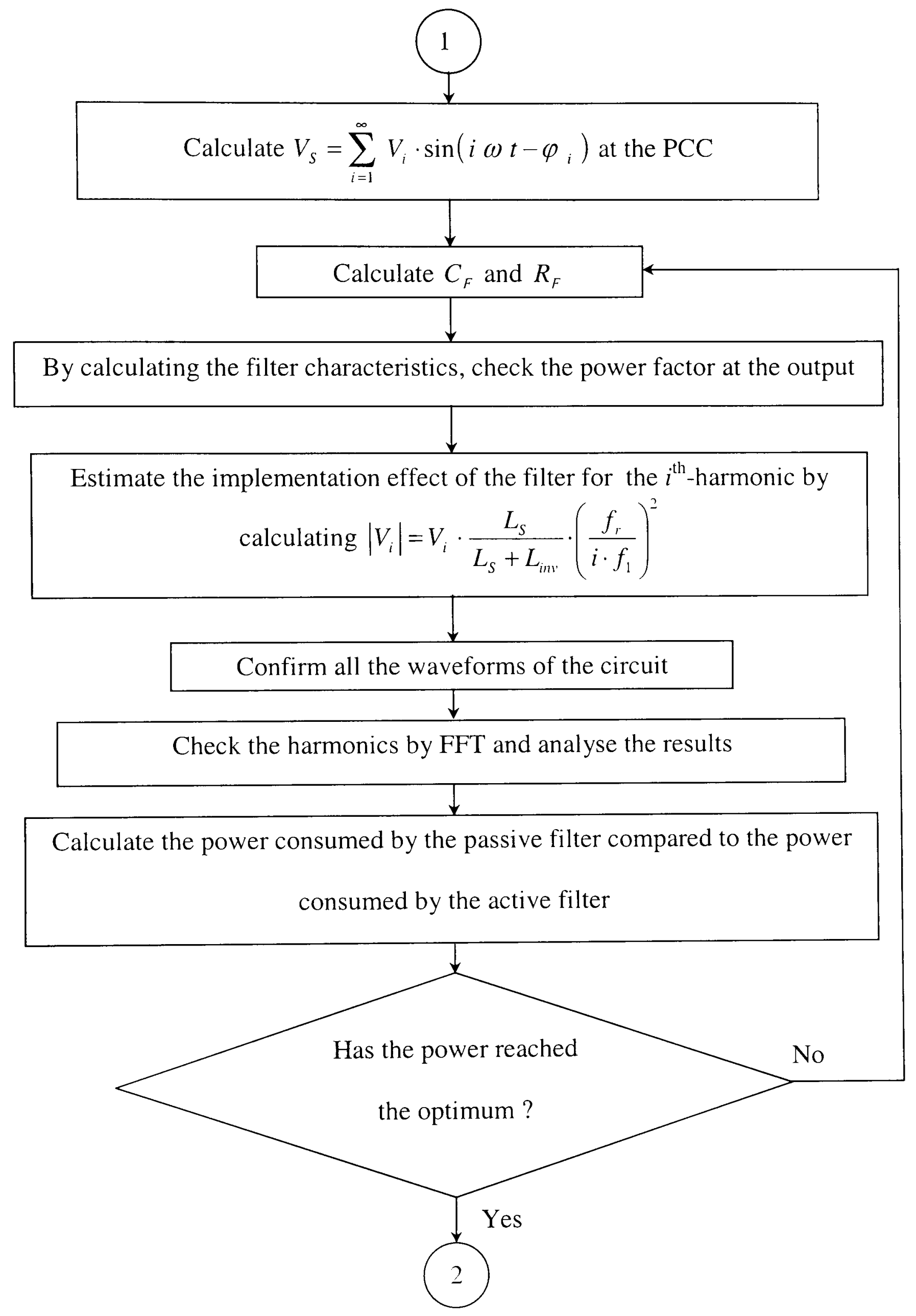

Fig.3.6 (Cont.): Flow chart of the final filter circuit design 


\subsection{Operation of the Proposed Circuit}

Assuming single-phase analysis for simplicity, the following equations can be written for the system of Fig.3.7:

$i_{s}=i_{l d}+i_{C o m p}$

The load current can be decomposed as

$i_{l d}=i_{1}+i_{h}+i_{1 \text { Re active }}$

hence,

Network

Impedance

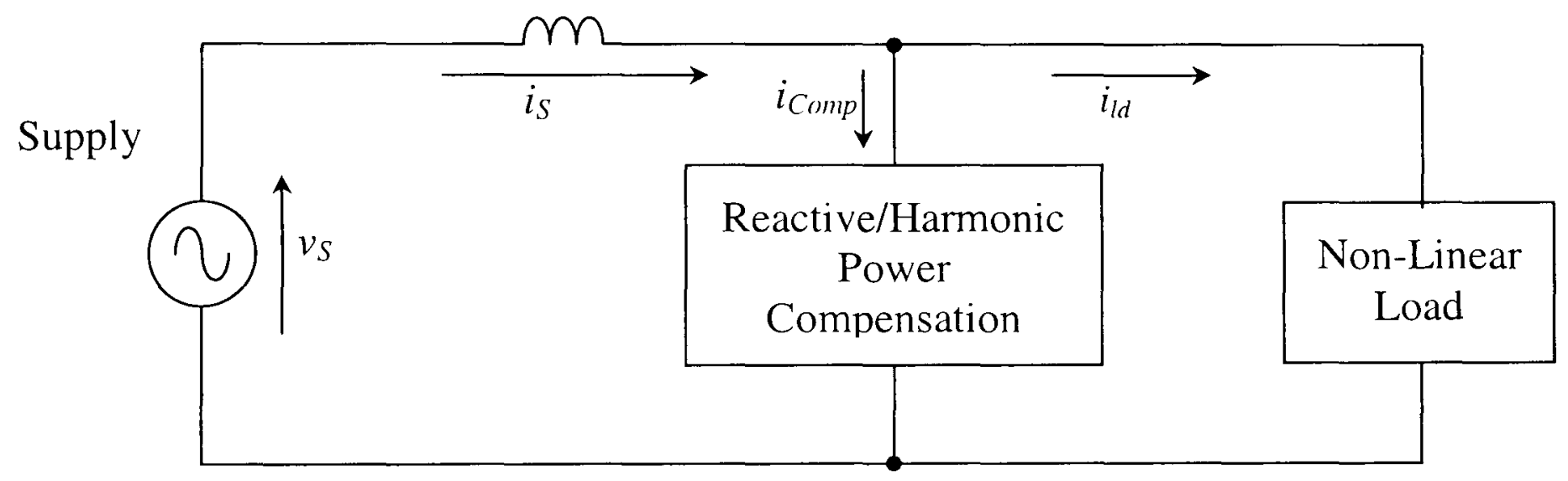

Fig.3.7: Basic structure of shunt-active power-filter configurations

$i_{S}=i_{1}+i_{h}+i_{1 \text { Re active }}+i_{\text {Comp }}$

The filter current has the form

$i_{\text {comp }}=-i_{h}-i_{1 \text { Re active }}$

which when substituting yields

$i_{S}=i_{1}+i_{h}+i_{1 \text { Reactive }}-i_{h}-i_{1 \text { Reactive }}$

and finally, 


$$
i_{S}=i_{1}
$$

As a conclusion, if the compensator current, $i_{\text {Comp }}$, is the same as the fundamental harmonic current, $i_{h}$, as well as the reactive component of current, $i_{1 \text { Re active }}$, then full compensation is achieved.

Referring to Fig.3.2, the current variation in the filter capacitor, $C_{F}$, during one sampling interval from $t_{k}$ to $t_{k+1}$, shown in Fig. 3.4, can be calculated as follows

$$
\Delta i_{\text {Comp }}=\frac{1}{L_{\text {Comp }}} \cdot \int_{t_{k}}^{t_{k}+1}\left(v_{l d}(t)-v_{F}(t)\right) \cdot d t
$$

which is very dependent on the equation

$$
\Delta i_{\text {Comp }}=i_{\text {Comp }}^{*}-i_{\text {Comp }}
$$

The pertinent variations in the filter capacitor voltage is given by

$$
\Delta v_{C_{F}}=\frac{1}{C_{F}} \cdot \int_{t_{k}}^{t_{k+1}} i_{F}(t) \cdot d t
$$

The charging and discharging of the filter capacitors can be solved in subsequent time intervals

$$
f_{S}=\frac{1}{t_{k+1}-t_{k}}
$$

The fundamental active power, $P_{F}$, for the filter circuit can be calculated as follows:

$$
P_{F}=\frac{V_{S}{ }^{2}}{R_{F}{ }^{2}+\left(\omega_{1} \cdot L_{F}-\frac{1}{\omega_{1} \cdot C_{F}}\right)^{2}} \cdot R_{F}
$$

In the same way, the fundamental reactive power, $Q_{F}$, is given by:

$Q_{F} \approx 1_{S}^{2} \cdot \omega_{1} \cdot C_{F}$ 
by assuming that $R_{F}{ }^{2}<<\left(\omega_{1} \cdot L_{F}-\frac{1}{\omega_{1} \cdot C_{F}}\right)^{2}$ and that $\omega_{1}^{2} \cdot L_{F} C_{F}<<1$.

\subsection{Exact Modelling of the Proposed Circuit}

For the purpose of controlling the proposed circuit and analysing its response, a mathematical modelling of the system is presented in this section. The model presented here will deal with the time and frequency domain analysis techniques. This modelling is in effect very useful in determining the internal characteristics of the filter and shows exactly the effects of introducing the passive filter circuit in between the inverter and the PCC of the supply.

Consider the circuit diagram of Fig.3.2, if the inverter is considered to operate satisfactorily in tracking the reference current set by its controller, it can be represented by a controllable current source. The same consideration can take place for the nonlinear load. This implies that the circuit of Fig.3.2 can be simplified to the one shown in Fig.3.8.

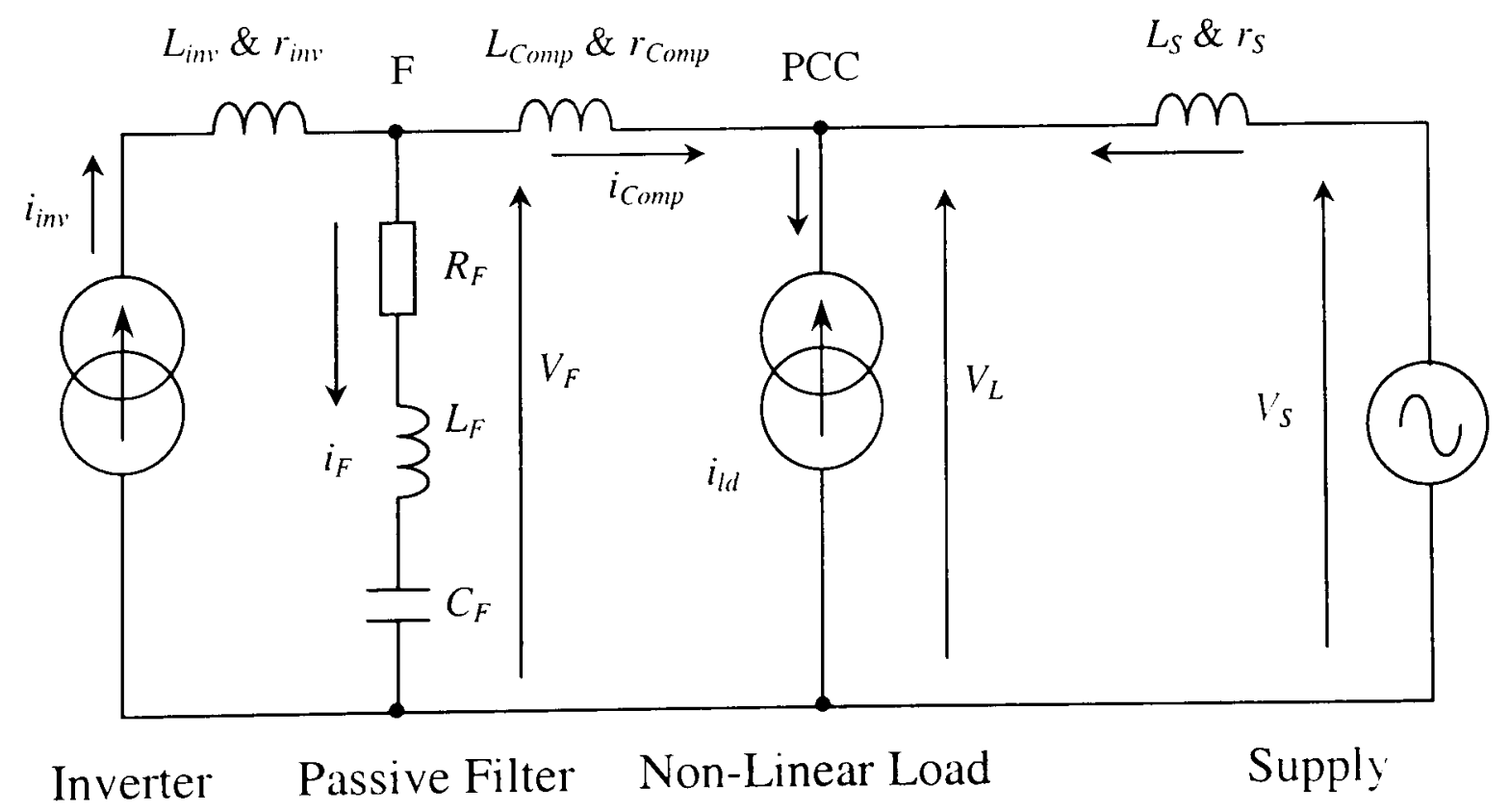

Fig.3.8: Modelling of the proposed circuit 
It is worthwhile to note that the current source shown here is the parallel superposition of all the current sources for the respective harmonics of either the load or the inverter currents.

The modelling process for the proposed system will consider a two-input/three-output system as the one shown in Fig.3.9. The supply voltage and the nonlinear load current are considered to be the inputs of the system, while the filter, supply and compensator currents are the outputs of the system. Any other variable in the system can be calculated by knowing these three values for the proposed system.

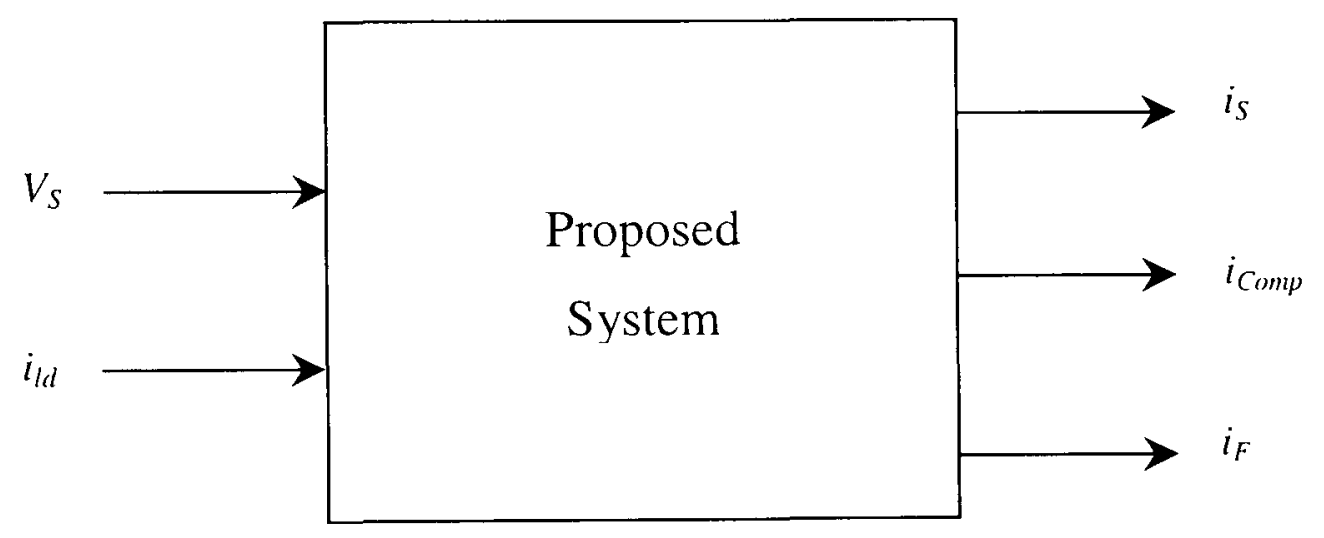

Fig.3.9: Input/output relationship between the filter variables

\subsubsection{Frequency-Domain Modelling}

The equivalent circuit diagram of Fig.3.8 is analysed in the following paragraphs to show the effects of each of the compensator components on the performance and operational characteristics of the system.

First of all consider the application of Kirchoff's current law at the point of common coupling. The current summation equation is the same as equation (3.17) and is repeated here for convenience.

$i_{\mathrm{S}}=i_{l d}+i_{\text {(omp }}$ 
The same applies to the following equation taking at the passive filter node, F.

$$
i_{F}=i_{i n v}+i_{C o m p}
$$

In order to complete the system model, a third equation is needed. This is obtained by applying Kirchoff's voltage law to the loop containing the supply voltage, supply inductance, compensator inductance and passive filter branch. The following equation results.

$$
v_{S}=r_{S} i_{S}+L_{S} \cdot \frac{d i_{S}}{d t}+r_{C o m p} i_{C o m p}+L_{C o m p} \cdot \frac{d i_{C o m p}}{d t}+R_{F} \cdot i_{F}+L_{F} \cdot \frac{d i_{F}}{d t}+\frac{1}{C_{F}} \int i_{F} \cdot d t
$$

Taking the Laplace-Transformation of equations (3.29) to (3.31) and rearranging, yields

$$
\begin{aligned}
& I_{S}-I_{C o m p}=I_{l d} \\
& -I_{C o m !}+I_{F}=I_{i n !} \\
& \left(r_{S}+s \cdot L_{S}\right) \cdot I_{S}+\left(r_{C o m p}+s \cdot L_{C o m p}\right) \cdot I_{C o m p}+\left(R_{F}+s \cdot L_{F}+\frac{1}{s \cdot C_{F}}\right) \cdot I_{F}=V_{S}+\frac{I_{F}(0)}{C_{F}}
\end{aligned}
$$

By assuming zero initial conditions, the last term of equation (3.34) can be neglected and the equation results in

$$
\left(r_{S}+s \cdot L_{S}\right) \cdot I_{S}+\left(r_{C o m p}+s \cdot L_{C o m p}\right) \cdot I_{C o m p}+\left(R_{F}+s \cdot L_{F}+\frac{1}{s \cdot C_{F}}\right) \cdot I_{F}=V_{S}
$$

Equations (3.32), (3.33) and (3.34) represent the s-domain model of the proposed system. In order to construct the block diagram representation of this system. these equations have to be rewritten in the following format.

$$
\begin{aligned}
& I_{s}=I_{(\mathrm{iml})}+I_{l d} \\
& I_{F}=I_{\text {cimm }}+I_{i m} .
\end{aligned}
$$




$$
I_{C o m p}=\frac{V_{S}-\left(r_{S}+s \cdot L_{S}\right) \cdot I_{S}-\left(R_{F}+s \cdot L_{F}+\frac{1}{s \cdot C_{F}}\right) \cdot I_{F}}{\left(r_{C o m p}+s \cdot L_{C o m p}\right)}
$$

The block diagram of Fig.3.10 is formed using the above three equations. It is to be noted that the system depends on the two inputs $\left(I_{l d}\right.$ and $\left.V_{S}\right)$ mentioned above in addition to the inverter current $\left(I_{i n v}\right)$, which is considered as an intermediate state between the input signals going through the controller and reference estimator block as well as through the inverter block.

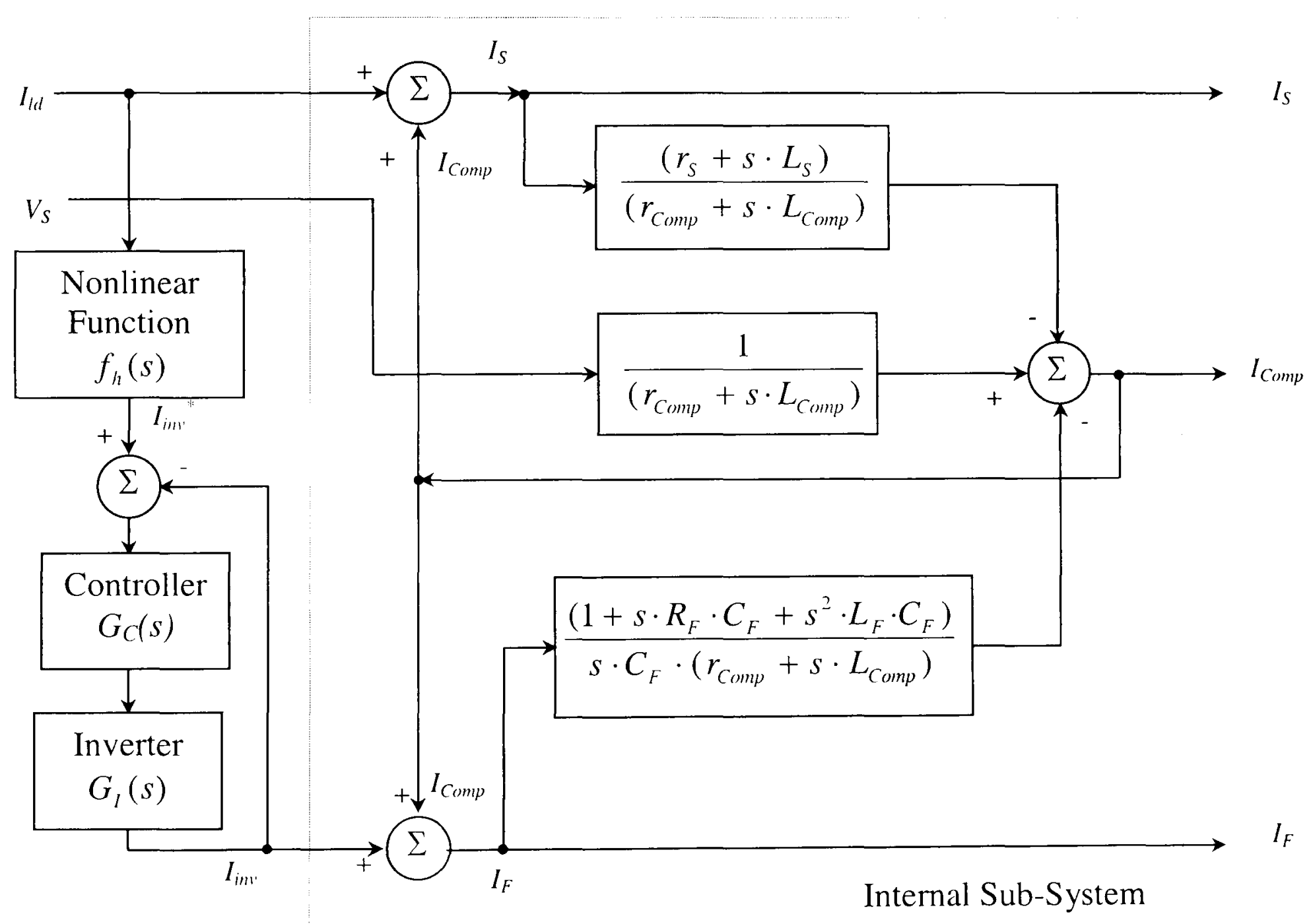

Fig.3.10: Control Block Diagram Representation of the Proposed System

It is worthwhile to note here that the inverter inductance. $L_{i n v}$, does not appear in this block diagram since the inverter is assumed to be a current source controlling the 
current through this inductor. This implies that the effect of this inductor is implicitly included in the inverter transfer function, $G_{I}(s)$, of Fig.3.10. The controller transfer function, $G_{r}(s)$ includes the response characteristics of the chosen controller, which is normally a Proportional-Integral (P-I) controller. The actual inverter current is compared to the desired reference, $I_{i n v}{ }^{*}(s)$, generated from a nonlinear harmonic current calculation function, $f_{h}(s)$. This reduces the order of the system (excluding the inverter, controller and the nonlinear function) to fourth order.

So far, the internal subsystem, formed in Fig.3.10, by the external passive circuit and the supply with three inputs and three outputs, can now be modelled in a transfer matrix expressed in the s-domain (see Appendix A). This matrix system model takes the form

$\underline{Y}(s)=\underline{G}(s) \cdot \underline{X}(s)$

where $\underline{Y}(s)$ is the output matrix expressed as

$$
\underline{Y}(s)=\left[\begin{array}{c}
I_{S}(s) \\
I_{F}(s) \\
I_{C o m p}(s)
\end{array}\right]
$$

The input vector $\underline{X}(s)$ is defined as

$$
\underline{X}(s)=\left[\begin{array}{c}
I_{l d}(s) \\
V_{S}(s) \\
I_{i m}(s)
\end{array}\right]
$$

The transfer function matrix is defined by the following

$$
\underline{G}(s)=\left[\begin{array}{lll}
G_{11}(s) & G_{12}(s) & G_{13}(s) \\
G_{21}(s) & G_{22}(s) & G_{23}(s) \\
G_{31}(s) & G_{32}(s) & G_{33}(s)
\end{array}\right]
$$

where the elements of this function are defined as follows. 


$$
\begin{aligned}
& G_{11}(s)=\frac{I_{s}(s)}{I_{l d}(s)}=\frac{1+s\left(R_{F}+r_{C o m p}\right) C_{F}+s^{2}\left(L_{F}+L_{C o m p}\right) C_{F}}{1+s\left(R_{F}+r_{C o m p}+r_{S}\right) C_{F}+s^{2}\left(L_{F}+L_{C o m p}+L_{S}\right) C_{F}} \\
& G_{12}(s)=\frac{I_{s}(s)}{V_{s}(s)}=\frac{s C_{F}}{1+s\left(R_{F}+r_{C o m p}+r_{S}\right) C_{F}+s^{2}\left(L_{F}+L_{C o m p}+L_{S}\right) C_{F}} \\
& G_{13}(s)=\frac{I_{s}(s)}{I_{\text {innv }}(s)}=\frac{-\left(1+s R_{F} C_{F}+s^{2} L_{F} C_{F}\right)}{1+s\left(R_{F}+r_{C o m p}+r_{S}\right) C_{F}+s^{2}\left(L_{F}+L_{C o m p}+L_{S}\right) C_{F}} \\
& G_{21}(s)=\frac{I_{F}(s)}{I_{l d}(s)}=\frac{-s C_{F}\left(r_{S}+s L_{s}\right)}{1+s\left(R_{F}+r_{C o m p}+r_{S}\right) C_{F}+s^{2}\left(L_{F}+L_{C o m p}+L_{S}\right) C_{F}} \\
& G_{22}(s)=\frac{I_{F}(s)}{V_{s}(s)}=\frac{s C_{F}}{1+s\left(R_{F}+r_{\text {Comp }}+r_{S}\right) C_{F}+s^{2}\left(L_{F}+L_{C o m p}+L_{S}\right) C_{F}} \\
& G_{23}(s)=\frac{I_{F}(s)}{I_{i m v}(s)}=\frac{s C_{F} \cdot\left[\left(R_{S}+r_{C o m p}\right)+s\left(L_{S}+L_{C \text { comp }}\right)\right]}{1+s\left(R_{F}+r_{C o m p}+r_{S}\right) C_{F}+s^{2}\left(L_{F}+L_{C o m p}+L_{S}\right) C_{F}} \\
& G_{31}(s)=\frac{I_{\text {Comp }}(s)}{I_{I d}(s)}=\frac{-s C_{F}\left(r_{S}+s L_{s}\right)}{1+s\left(R_{F}+r_{C o m p}+r_{S}\right) C_{F}+s^{2}\left(L_{F}+L_{C o m p}+L_{S}\right) C_{F}} \\
& G_{32}(s)=\frac{I_{\text {Comp }}(s)}{V_{s}(s)}=\frac{s C_{F}}{1+s\left(R_{F}+r_{\text {Comp }}+r_{S}\right) C_{F}+s^{2}\left(L_{F}+L_{C o m p}+L_{S}\right) C_{F}} \\
& G_{33}(s)=\frac{I_{\text {Comp }}(s)}{I_{i m v}(s)}=\frac{-\left(1+s R_{F} C_{F}+s^{2} L_{F} C_{F}\right)}{1+s\left(R_{F}+r_{C o m p}+r_{S}\right) C_{F}+s^{2}\left(L_{F}+L_{C o m p}+L_{S}\right) C_{F}}
\end{aligned}
$$

The characteristic equation of the system is defined as

$$
\Delta=1+s\left(R_{F}+r_{\text {Comp }}+r_{S}\right) C_{F}+s^{2}\left(L_{F}+L_{C o m p}+L_{S}\right) C_{F}
$$

It is clear that this value is the same as the denominator of the above equations. The characteristic equation determines the performance of the system under different operating conditions. It is clear that for any values of resistors, inductors and capacitor, the poles of the characteristic equation lie in the left-hand side of the splane, which gives an early indication of the stability of the system. 
The inverter is assumed to be represented with a constant gain for low frequency operation $(<5000 \mathrm{~Hz}$ for a $20 \mathrm{kHz}$ switching frequency). For higher frequencies. it possesses low pass filter characteristics. The transfer function, $G_{l}(s)$, can be approximated as in the following transfer function.

$G_{I}(s)=\frac{K_{I}}{\left(1+\tau_{I} \cdot s\right)}$

where $K_{I}$ is the inverter gain and $\tau_{l}$ is the inverter delay time constant.

Introducing the effects of the terms $\left(G_{c}(s) \cdot G_{I}(s)\right)$ in conjunction with the nonlinear function, $f_{h}(s)$, into the above model would reduce the input variables to only two variables.

The inverter reference current was modelled earlier in equations (3.3) to (3.7). The Laplace transform of the function in equation (3.3) will provide the desired nonlinear function for the reference current computation.

$$
I_{i n v}(s)=\sum_{i=1}^{N_{H} \text { Harmonics }}\left(\frac{\sqrt{2} \cdot I_{l d h_{i}}}{m_{L C L i}}\right) \cdot\left(\frac{\left(i \omega_{1}\right)^{2}}{\left(s+\varphi_{i n v i}^{*}\right)^{2}+\left(i \omega_{1}\right)^{2}}\right)
$$

where, the values of $m_{L C L_{i}}$ and $\varphi_{i n_{i}}^{*}$ are calculated from equations (3.5) and (3.6).

Using the above models for the various transfer functions, it is now possible to study the performance of each of the system blocks. The frequency domain analysis for the various transfer functions is presented in Fig.3.11 to Fig. 3.15 calculated using the system parameters given in appendix B and presented later on in this chapter. It is to be noted that 
$G_{12}(s)=G_{22}(s)=G_{32}(s)$

$G_{13}(s)=G_{33}(s)$

$G_{21}(s)=G_{31}(s)$

which involve the plotting of only five transfer functions of the nine ones available. The negative signs in the transfer functions, $G_{13}(s), G_{21}(s), G_{31}(s)$ and $G_{33}(s)$, indicate a change of phase of $180^{\circ}$ (i.e., the two currents are in the reverse direction to their assumed ones). All the system poles are the same and are in the left-hand-side of the s-plane.

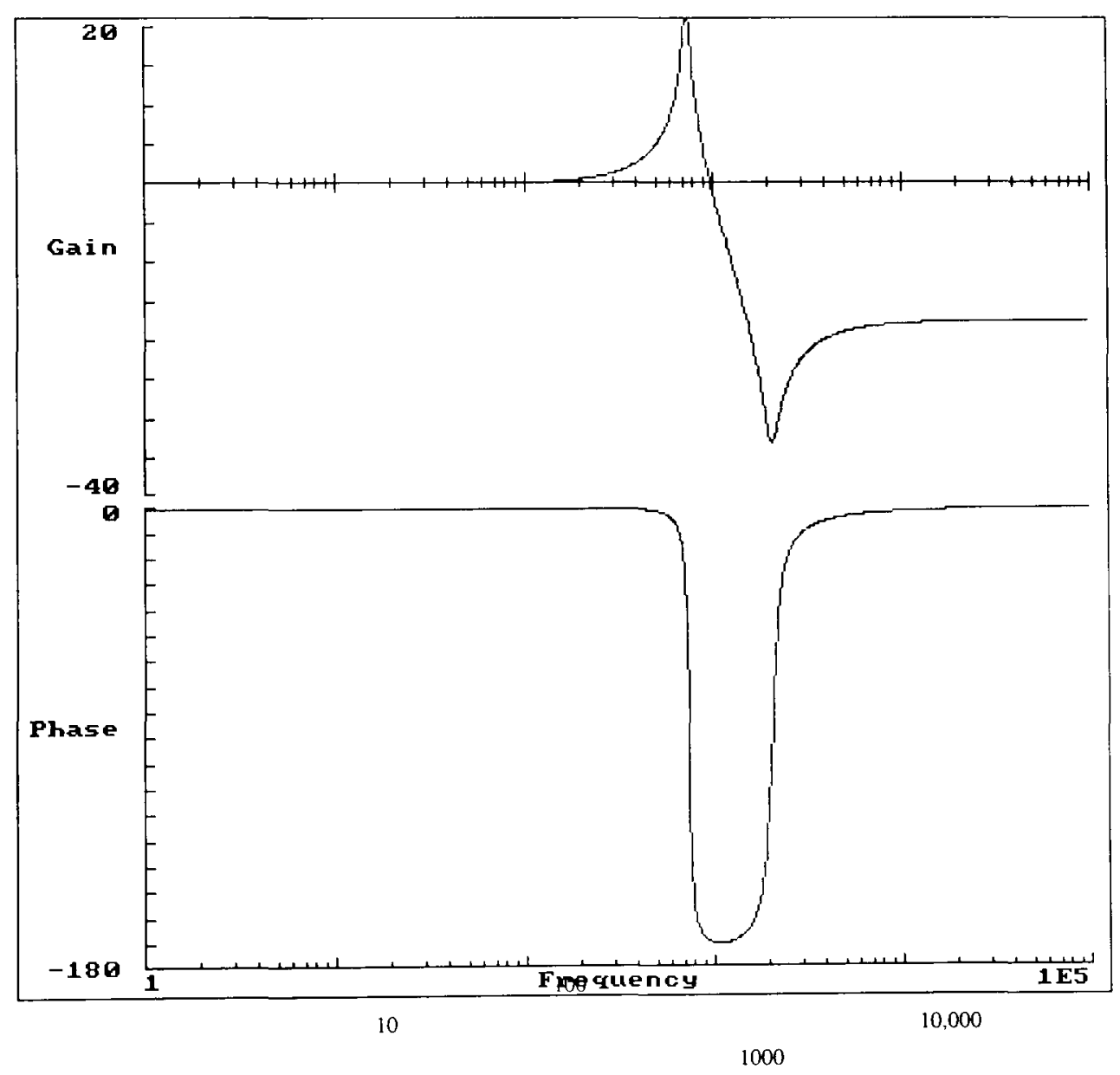

Fig.3.11 : Bode diagram of the transfer function $G_{1 l}(s)$

The frequency domain Bode plots are performed for the open-loop transfer functions. Each ligure shows the gain and phase relationships of the transfer function with respect to the frequency changing from $1 \mathrm{~Hz}$ up to $100 \mathrm{kHz}$. All the five transfer functions exhibit resonance around $790 \mathrm{~Hz}$, which is beyond the $15^{\text {th }}$ harmonic of the 
power system. The inverter switching frequency is of course much larger than this value and is around 20 times higher which makes it in the safe side.

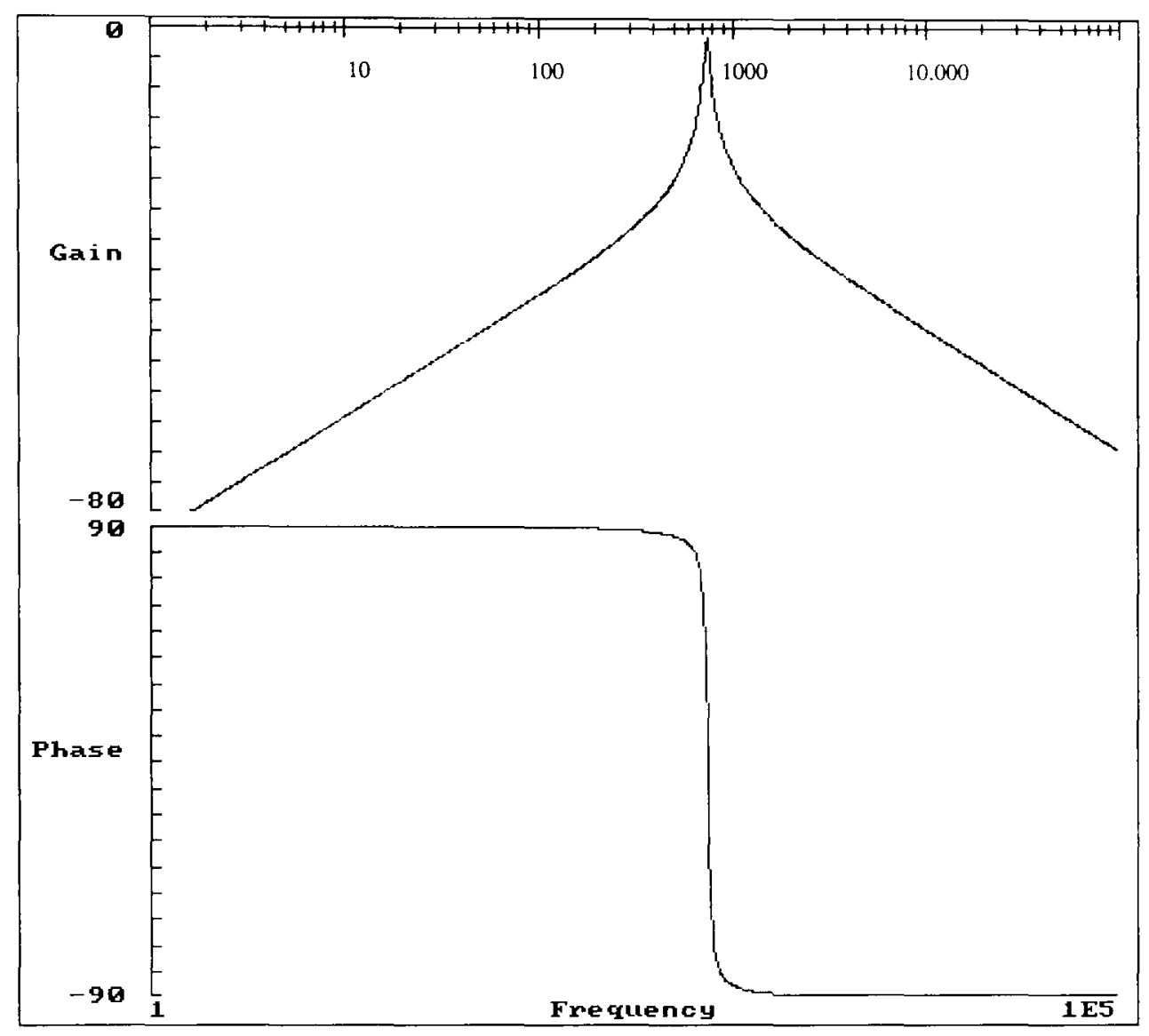

Fig.3.12: Bode diagram of the transfer function $G_{12}(s)$

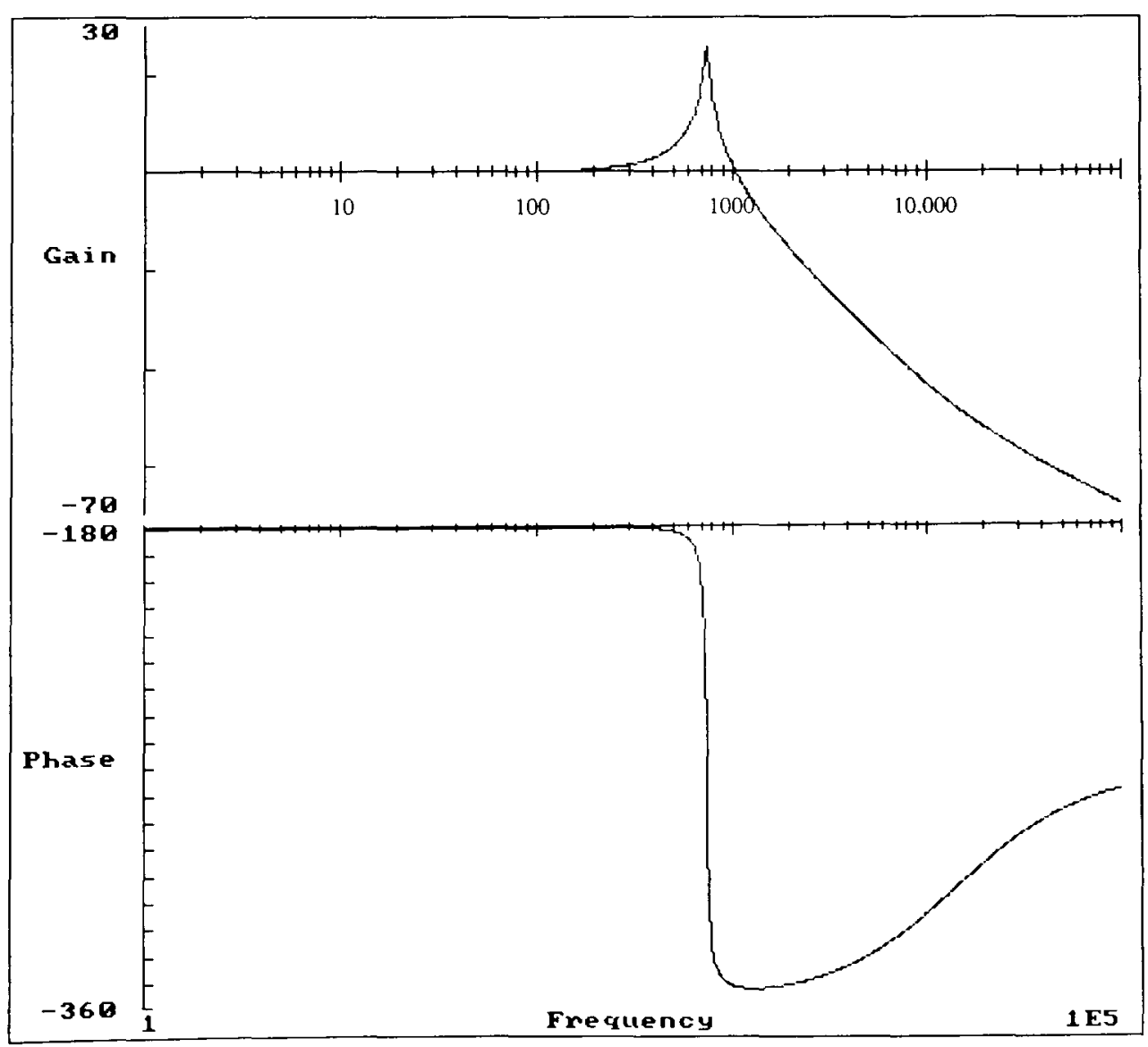

Fig.3.13: Bode diagram of the transfer function $G_{I .3}(s)$ 


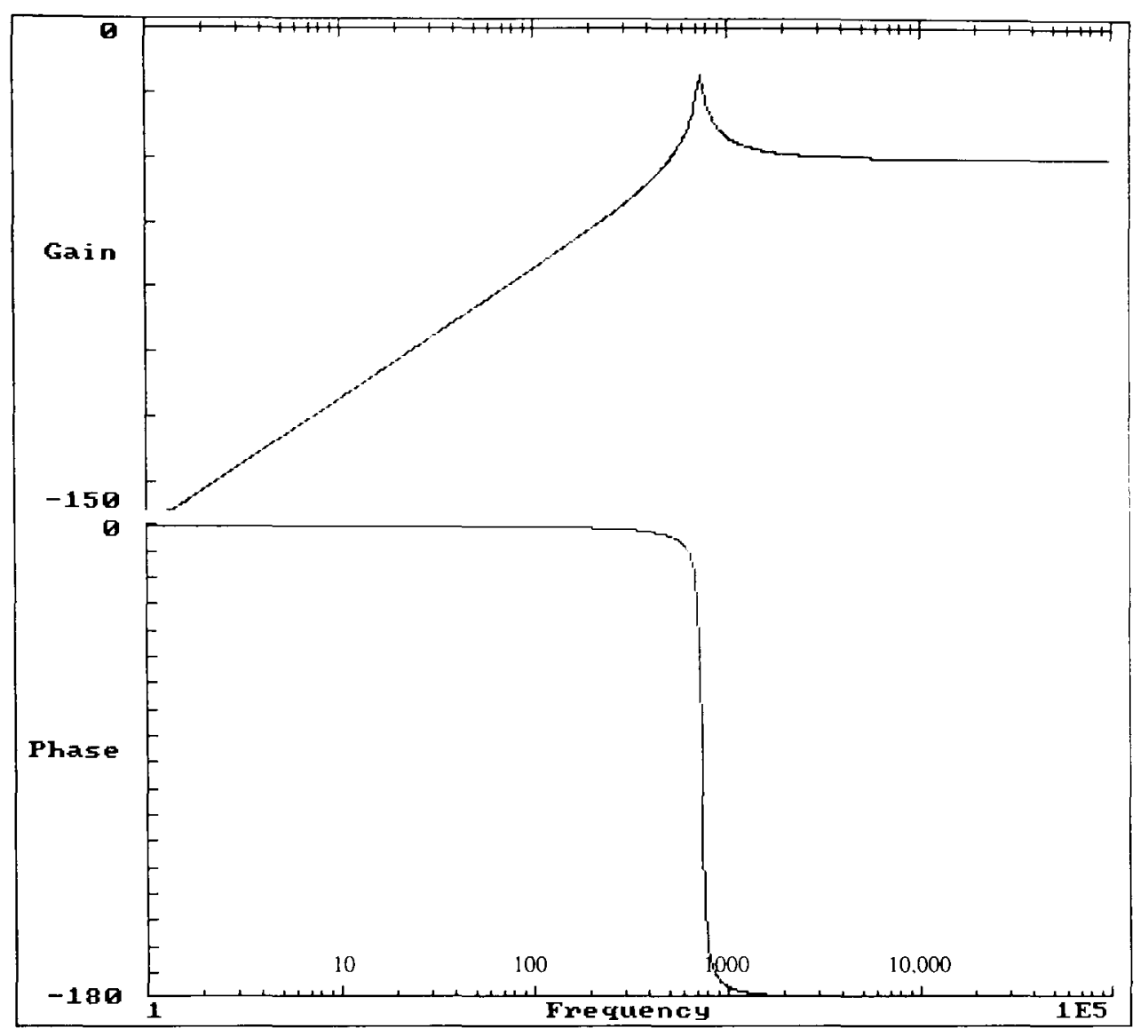

Fig.3.14: Bode diagram of the transfer function $G_{21}(s)$

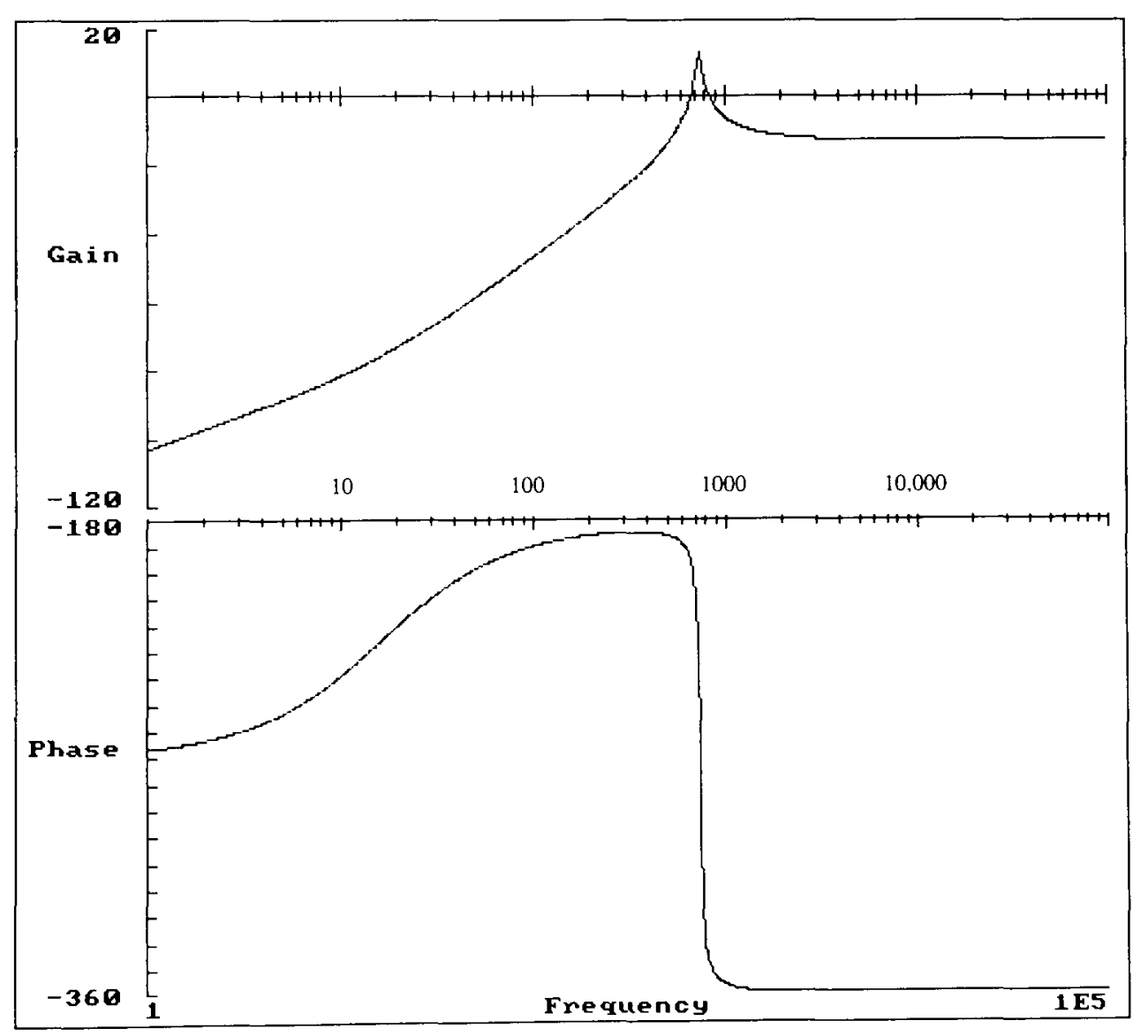

Fig.3.15: Bode diagram of the transfer function $G_{32}(s)$ 
It is also noted from the curves that the systems at higher frequencies will manifest the low-path filter characteristics by at least $20 \mathrm{~dB}$ which is one tenth of the signal and henceforth the high switching ripple from the inverter side will not at all be transmitted to the power system to cause electromagnetic interference with communication systems or malfunction of protective devices.

Fig.3.13 showing the transfer function the supply current to the inverter current, $G_{13}(\mathrm{~s})$, implies that the proposed filter system is intrinsically capable of passing the inverter current to the supply side for the range of frequencies below $1 \mathrm{kHz}(3 \mathrm{~dB}$ cutoff frequency). This frequency enables the inverter to control the supply current up to and including the $20^{\text {th }}$ harmonic, which is very sufficient for power system applications at low or medium voltages. As mentioned above, this Bode plot also shows that the filter system will ensure that the switching harmonics (normally 16$20 \mathrm{kHz}$ ) will not reach the supply side. The attenuation at $10 \mathrm{kHz}$ is approximately $48 \mathrm{~dB}$.

The phase shift angle of the Bode plot of the same transfer function in Fig.3.13 shows that the system does not exhibit any considerable phase shifts for current signals generated from the inverter up to frequencies above $650 \mathrm{~Hz}$ which is equivalent to the $13^{\text {th }}$ harmonic. For higher frequencies, the passive filter circuit exhibits a considerable phase-shift between the input inverter current and the output supply current. However this phase shift can be accounted for in the controller design which is not shown in the diagrams. This is presented in equation (3.54), which includes a phase correction factor. $\varphi_{i n w_{i}}{ }^{*}$, for the current reference, which is expressed in equation (3.6). 


\subsubsection{State-Space Modelling}

In order to put the system in the state space model, the instantaneous time equations (3.29) to (3.31) defined in the previous section, can be manipulated. The system is seen to be of second order and needs two state variables to be defined. Taking the system states as being $x_{1}$ and $x_{2}$. The variable $x_{1}$ is defined as being the compensator current, $i_{C o m p}$, while the variable $x_{2}$ is defined as its time derivative.

Putting the system in the general state space format

$\underline{X}^{\bullet}=\underline{A} \underline{X}+\underline{B}_{0} \underline{U}+\underline{B}_{1} \underline{U}^{\bullet}+\underline{B}_{2} \underline{U}^{\bullet}$

where the superscripted dot defines the time derivative of the variable and the state vector $\underline{X}$ is defined as

$\underline{X}=\left[\begin{array}{l}x_{1} \\ x_{2}\end{array}\right]$

the input vector $\underline{U}$ is

$\underline{U}=\left[\begin{array}{c}i_{l d} \\ i_{i m l} \\ v_{S}\end{array}\right]$

The state matrix is

$$
A=\left[\begin{array}{cc}
0 & 1 \\
\frac{-1}{\left(L_{l}+L_{S}+L_{C o m p}\right) \cdot C_{F}} & \frac{-\left(R_{F}+r_{S}+r_{\text {Comp }}\right)}{\left(L_{F}+L_{S}+L_{\text {Comp }}\right)}
\end{array}\right]
$$

The input coefficient matrices are

$$
\underline{B}_{0}=\frac{1}{\left(L_{F}+L_{S}+L_{C o m p}\right)} \cdot\left[\begin{array}{ccc}
0 & 0 & 0 \\
0 & 0 & \frac{1}{C_{F}}
\end{array}\right]
$$




$$
\begin{aligned}
& \underline{B}_{1}=\frac{1}{\left(L_{F}+L_{S}+L_{\text {Comp }}\right)} \cdot\left[\begin{array}{ccc}
0 & 0 & 0 \\
-r_{S} & 1 & -R_{F}
\end{array}\right] \\
& \underline{B}_{2}=\frac{1}{\left(L_{F}+L_{S}+L_{\text {Comp }}\right)} \cdot\left[\begin{array}{ccc}
0 & 0 & 0 \\
-L_{S} & 0 & -L_{F}
\end{array}\right]
\end{aligned}
$$

The output equation is defined as

$$
\underline{Y}=\underline{C} \underline{X}+\underline{D} \underline{U}
$$

The output matrix has the form

$$
\underline{C}=\left[\begin{array}{ll}
1 & 0 \\
1 & 0 \\
1 & 0
\end{array}\right]
$$

and the input feedback matrix is given by

$$
\underline{D}=\left[\begin{array}{lll}
1 & 0 & 0 \\
0 & 0 & 0 \\
0 & 0 & 1
\end{array}\right]
$$

As mentioned earlier for the s-domain model, the effects of the controller and inverter transfer functions can also be added to the above system. The inclusion of the nonlinear current computation function will then determine the total transfer function of the system by eliminating the need for the $2^{\text {nd }}$ input $\left(i_{\text {inv }}\right)$.

\subsection{Effect of Filter Connection to the Power System}

The following discussion outlines the fundamental compensation principle of the passive filter connected to the compensator inverter. The mains network and different compensation device circuits are analysed and discussed in detail, in relation to Fig.3.16, in order to determine the effects of filter insertion into the power system. 


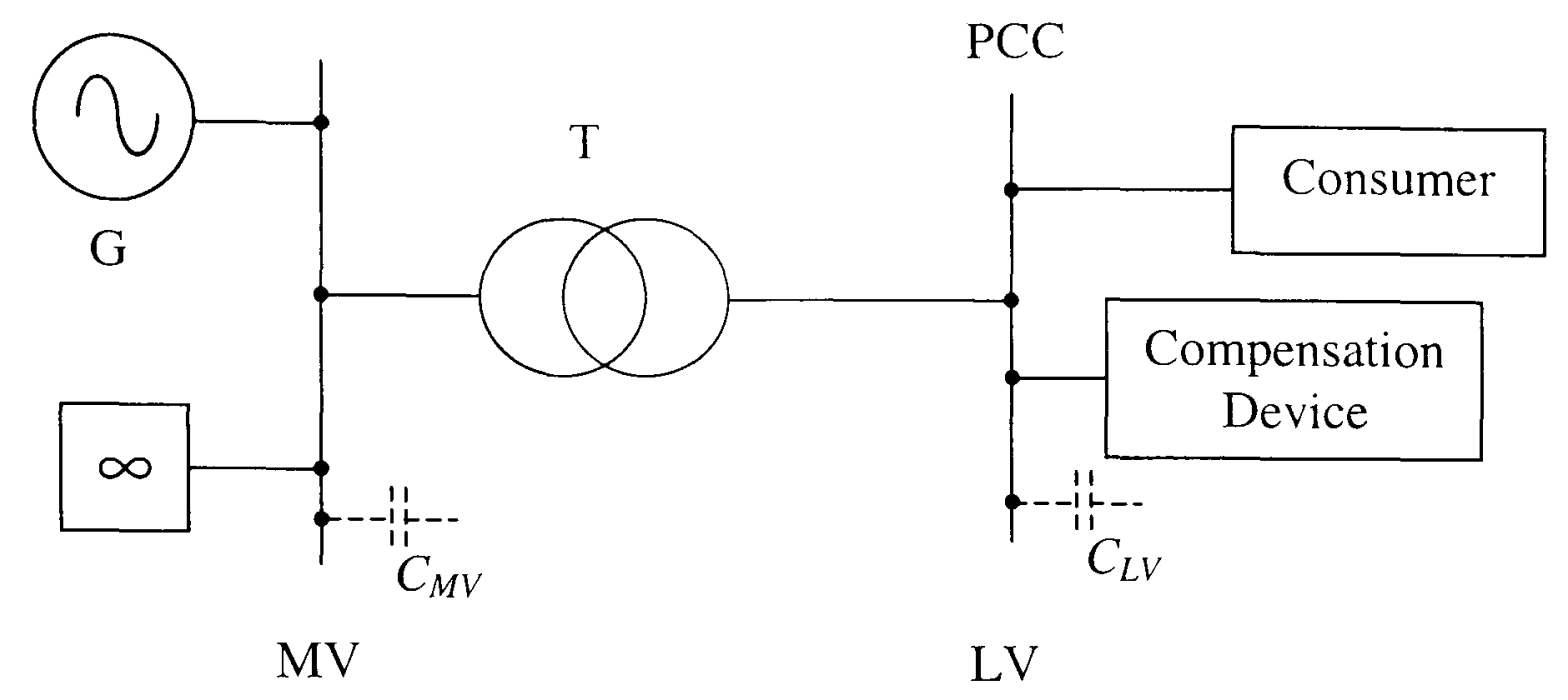

Fig.3.16: Network Diagram, Compensation and Filter Circuit Consideration

Fig. 3.16 shows a consumer load and compensation devices connected at the point of common coupling (PCC) to the secondary bus-bar of a distribution transformer $(\mathrm{T})$. The operation of this load must not affect other appliances connected to the same low voltage (LV) or medium voltage (MV) bus-bars. This requires compliance with the connection rules of the supply-company and the international harmonic and reactive power compatibility levels. In order to ensure the correct operation, the user has to calculate his own permissible interference-emissions with the given data of the mains feeders, transformers and short-circuit levels provided by the supply company. The following parameters are listed to estimate the effects of the load connection.

1. Network plan of the new customer installation, rated power, maximum power, operating times.

2. The types of harmonic generating loads, their ratings and their occurrence of the load must be known.

3. The Supply company must provide the transformer ratings and the short-circuit power level at the MV side of the transformer, in order to define the network impedance.

4. The customer must take the power factor correction into consideration. 


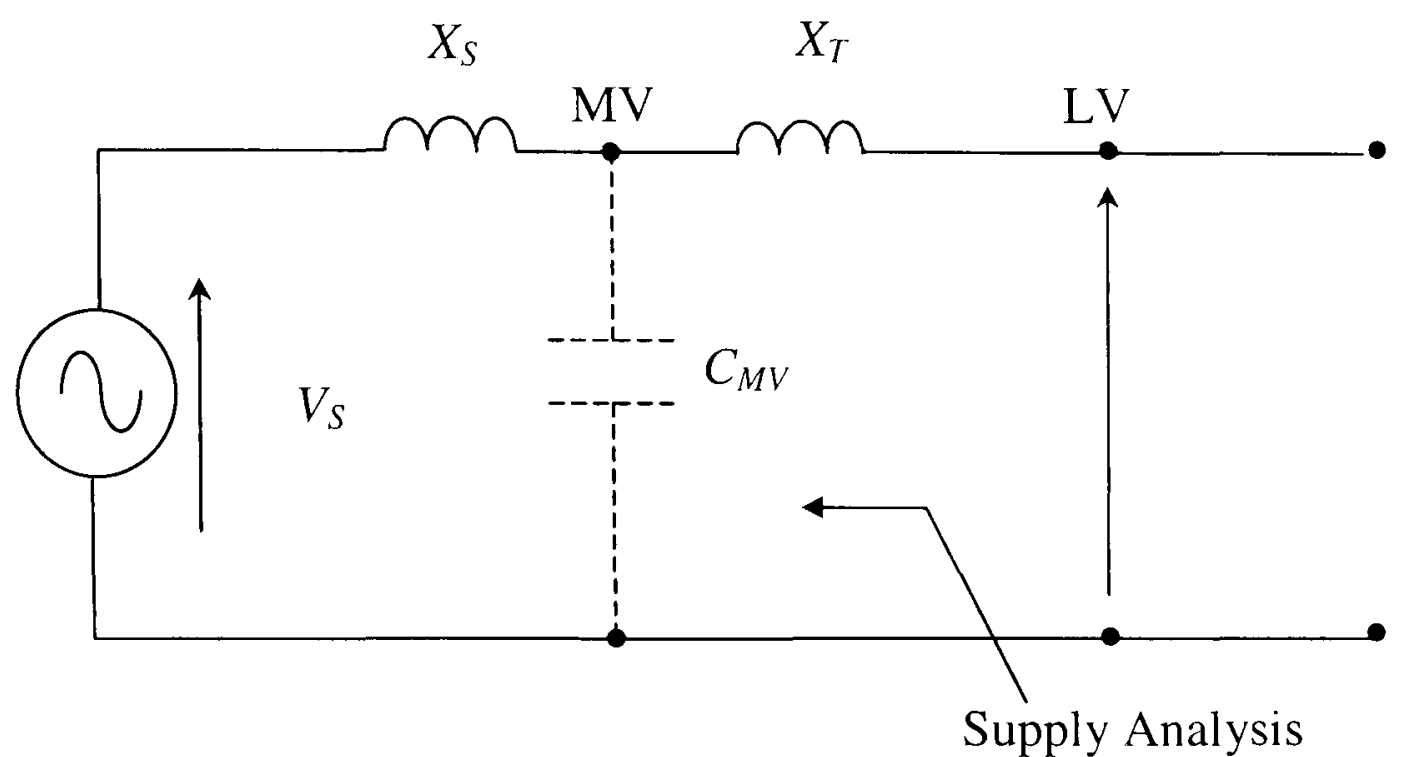

(a) Single compensation on the MV bus-bar

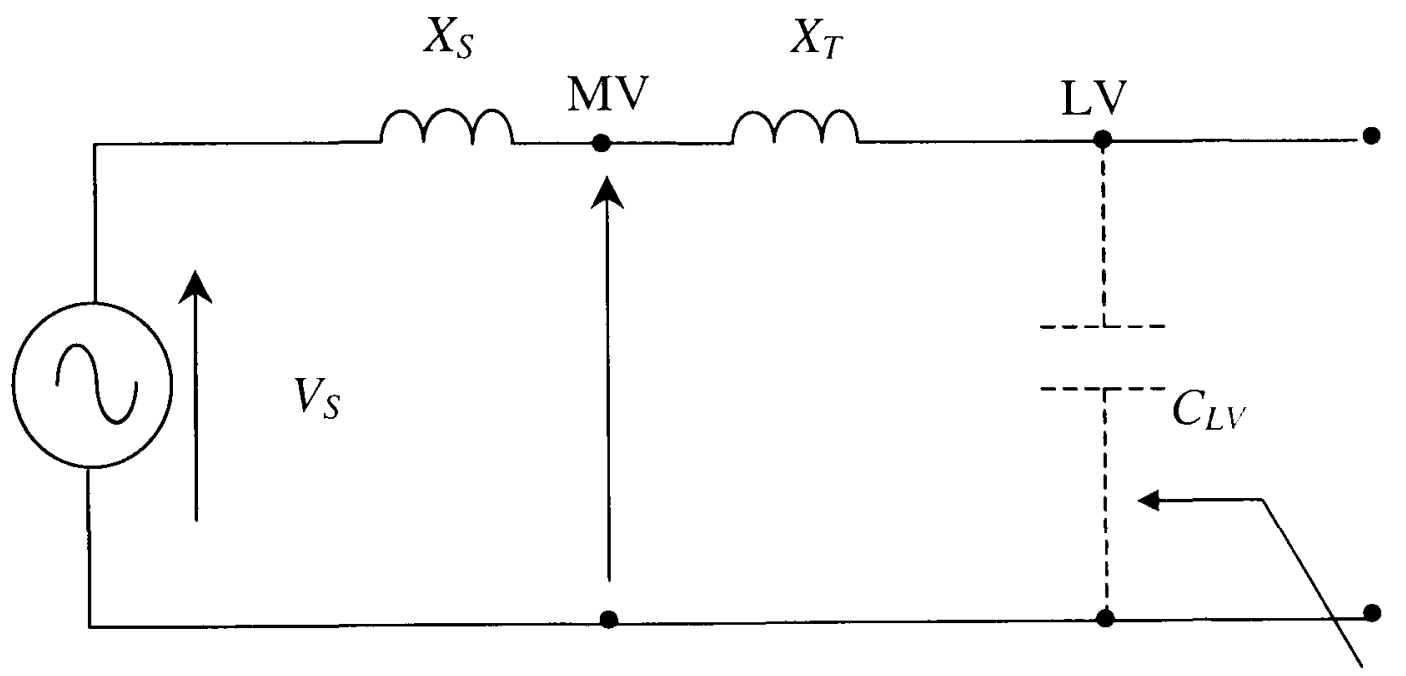

Supply Analysis

(b) Single compensation on the LV bus-bar

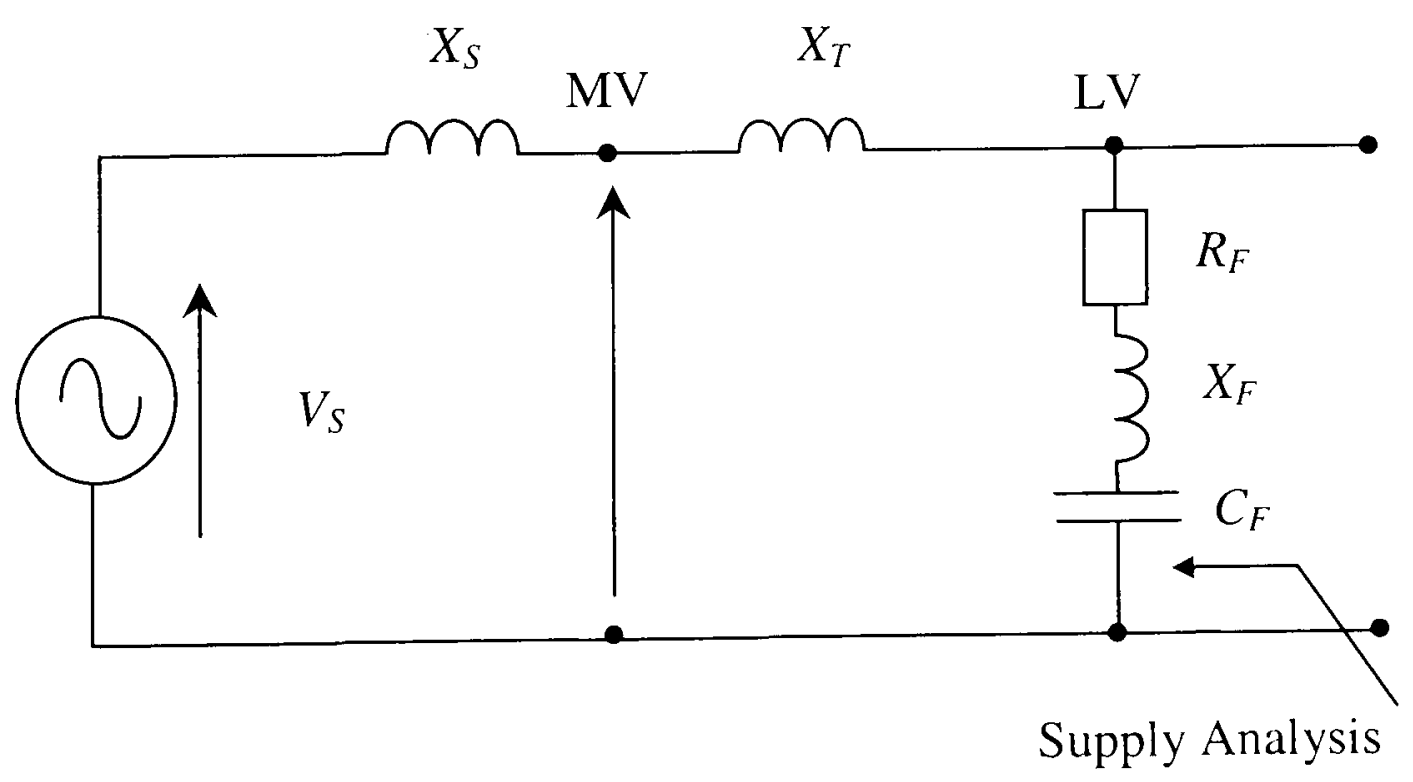

(c) Consideration of the Filter Circuit

Fig.3.17: Equivalent circuit for different compensation cases 
In order to appreciate the effect of the insertion of the proposed passive part of the filter at the PCC, consider the power system shown in Fig.3.16, when analysed with reference to the MV bus-bar, as shown in Fig.3.17-a. The arrow indicates the direction of analysis. In this figure, a MV compensation capacitor, $C_{M V}$, is inserted at the MV bus-bar. The effect of the supply impedance is taken into consideration for the parallel resonance occurring with this $\mathrm{MV}$ capacitor, $C_{M V}$.

First, the network impedance $X_{S}$ is determined from Fig.3.17-a:

$$
X_{s}=\omega_{1} L_{s}
$$

Alternatively, the network impedance $X_{S}$ can be calculated from the short circuit power $S_{s c}{ }^{\infty}$ at the PCC as:

$$
X_{s}=\frac{V_{S}^{2}}{S_{s c}^{\infty}}
$$

The resonance in this case is uncontrollable and can occur at any frequency depending on the ever-changing supply impedance.

Consider the case of a $\mathrm{LV}$ capacitor, $C_{L V}$, insertion in the power system as shown in Fig.3.17-b. The main influence on the network impedance in this case is the reactance of the transformer, $X_{T}$, and by neglecting the transformer resistance, the transformer reactance can be given as

$$
X_{T}=\frac{V^{2} S_{T}^{\text {Rated }}}{v_{s c}^{\text {Rated }}}
$$

The variation is, however, in this reactance is very dependent on the different loads and network topologies. If only the fundamental reactive power, $Q_{C}$, compensation is 
considered, then the capacitance and network-inductance form a parallel resonant circuit. The ratio of the resonance frequency of the system to the fundamental frequency can be calculated from

$$
h_{p}=\frac{f_{r}}{f_{1}}=\sqrt{\frac{S_{S C}^{\infty}}{Q_{C}}}
$$

The current may increase in connection with the parallel resonance and, consequently, a high harmonic voltage $v_{s}^{p}$ appears across the network reactance and is given by

$$
V_{S_{i}}^{P}=Q F \cdot i \cdot X_{s} i_{F i}^{P}
$$

where $Q F$ is the quality factor of the resonance circuit and $p$ is the ratio (in percent) of the reactance of the equivalent system inductance to that of the capacitor. In other words, $p$ is a characteristic for the degree of harmonic absorption at network frequency and is normally equal to $5.67 \%, 7 \%$ and $14 \%$. It is defined as

$$
p=\frac{X_{L}}{X_{C}}
$$

To avoid the parallel resonance, an inductance, $L_{F}$, and a damping resistor, $R_{F}$, can be connected in series to the filter capacitor, $C_{F}$, (Fig.3.17-c), then the required inductance is defined by

$$
L_{r}=\frac{1}{\omega^{2}{ }_{r} C}=\frac{1}{h^{2}, \omega^{2}{ }_{1} C}
$$

where, $L_{r}=L_{S}+L_{T}+L_{F}$, which alters the value of the resonance frequency of the system in this case.

The relationship between $p$ and the resonant frequency $f_{r}$ given by

$$
i_{r}=f_{1} \cdot \frac{1}{\sqrt{p}}
$$


The effect of this compensation case is that the resonance frequency of the supply is changed and is somehow controllable and the value of $L_{F}$ can be chosen to shift the resonance frequency away from the band of interest.

\subsection{Numerical Example}

In order to gain further understanding of the design of the proposed active filter system, at this stage, it is best to consider a numerical example of the computation of the filter parameters and the corresponding protective measures that ensure the operation of the proposed system. The inverter and passive filter parameters need to be specified from the design equations outlined earlier in this chapter.

\subsubsection{Connection of a motor drive load to the low voltage power system}

In an installation, it is intended to connect a motor drive, whose direct current motor is supplied from a rectifier. The following data is given.

Transformer rating power $630 \mathrm{KVA}$, short circuit voltage $4 \%$,

Voltage at the PCC $V_{s}=400 \mathrm{~V}$, short circuit power $S_{s c}^{\infty}=500 M V A$,

Maximum network power $S_{\text {Mains }}=0.5 \mathrm{MVA}$

Connection power of the consumer $S_{\text {Demand }}=0.3 \mathrm{MVA}$,

Power of the drive $P=20 \mathrm{~kW}$, efficiency $\eta=0.95, \cos \varphi=0.95$

Pulse number of the rectifier $p=6$ for inductive smoothing

- Calculation of the short circuit power at the PCC:

$X_{S C(P())}=\frac{1}{S_{s i}}+\frac{v_{S C}}{S_{T}^{\text {Rated }}}=\frac{100 \%}{500 \mathrm{MLA}}+\frac{4 \%}{0.63 \mathrm{MLA}}=6.5 \% / \mathrm{MLA}$ 


$$
S_{s c(P C C)}^{\infty}=\frac{1}{X_{s c(P C C)}}=\frac{100}{6.5 \% / M V A}=15.38 \mathrm{MVA}
$$

- Calculation of the Harmonic disturbance factor caused by a single customer appliance for each harmonic order (Table. 3.2):

$$
B_{h}=\frac{z_{h} \cdot \sum k_{h_{i}} \cdot i_{h_{i}} \cdot S_{\text {Comnerted } i}^{\text {Rated }}}{V_{h_{p e c}}}
$$

Table. 3.2: Calculation of the disturbance factor

\begin{tabular}{|c|c|c|c|c|c|}
\hline $\boldsymbol{h}$ & $\boldsymbol{k}_{\boldsymbol{h}}$ & $\boldsymbol{i}_{\boldsymbol{h}}$ & $\boldsymbol{V}_{\boldsymbol{h}, P C C}$ & $z_{h}=\boldsymbol{h} . \boldsymbol{X}_{S C(P C C)}$ & $\mathrm{B}_{\mathrm{h}}$ \\
for $20 \mathrm{KV}$ & $\%)$ & $(\%)$ & $\% / \mathrm{MVA}$ & \\
\hline 5 & 0.5 & 27 & 6 & 32.5 & 0.038 \\
7 & 0.3 & 11 & 5 & 45.5 & 0.022 \\
11 & 0.2 & 9 & 3.5 & 71.5 & 0.02 \\
13 & 0.2 & 6 & 3 & 84.5 & 0.02 \\
\hline
\end{tabular}

For the $20 \mathrm{~kW}$ Motor drive is

$$
\begin{aligned}
& S_{\text {Connectled }}^{\text {Rated }}=\frac{P}{\eta \cdot \cos \phi}=22.2 \mathrm{kVA} \\
& \frac{S_{\text {Connuected }}^{\text {Rated }}}{S_{S C}^{\infty}}=\frac{22.2 \mathrm{KVA}}{15.38 \mathrm{MVA}}=0.00144
\end{aligned}
$$

The sum of three harmonic currents, $I_{L V, h}, I_{M V, h}, I_{H V, h}$, produce the resultant harmonic voltages in the power system. In order to maintain the EMC, the resultant voltage must be less than or equal to the EMC level for the corresponding harmonic. Each of these harmonic currents therefore contributes a proportion towards the harmonic loading of the power system. Then the network voltage level factor, $k_{k}$ for the low voltage system can be given as

$k_{N}=0.2-0.3$ 
In order to ensure that the permissible sum current is not exceeded, the proportion assigned to an individual customer is determined by the connection factor, $k_{C}$, which is a ratio of connection power to the main power for which the system is designed.

$k_{C}=\frac{S_{\text {Demand }}}{S_{\text {Main. }}}=\frac{300 k V A}{500 k V A}=0.6$

Evaluation of the interference transmission, which is required as $B_{\max }<k_{C} \cdot k_{N}=0.18$ is satisfied for all harmonic order.

where $B_{\max }>B_{h}$, then the connection may be approved.

\subsubsection{Compensator parameter determination}

In order to perform the design of the proposed compensator for the above load, a 3phase compensator is needed. This implies that the current rating for a $400 \mathrm{~V}$ line voltage per phase is approximately $32 \mathrm{~A} \mathrm{rms}$. The peak value of the current in that case would be around 45A. A compensator tolerating this rating is to be designed.

In this thesis, the proposed system is tested on a single-phase basis, which implies that the loading of the system is around 45A peak. Some of the loads necessitate 110$130 \%$ harmonics and reactive power compensation of the rated fundamental current, which brings the filter rating to around 50A. The single-phase active filter is then to be designed for this current rating as in the following paragraphs.

For the given compensation current of around $50 \mathrm{~A}$ at a supply voltage of $230 \mathrm{~V}$ (single-phase), $50 \mathrm{~Hz}$, the power factor of the plant needs to be increased from 0.8 to a minimum of 0.95 . The harmonics of the power system have been measured. 
In accordance with the future development, the $5^{\text {th }}, 7^{\text {th }}$ and $13^{\text {th }}$ harmonics need to be specifically eliminated.

In order to proceed with the design process of the prototype active/passive shunt power filter, it is required to meet the specification procedure. These specifications will be considered in the following sections.

\section{Inverter-Characteristics needed to be considered}

1. The switching frequency of the inverter $\left(f_{\text {Switching }}\right)$

2. The supply voltage across of the inverter $\left(V_{D C}\right)$

3. The blocking voltage of the inverter $\left(V_{\text {Blocking }}\right)$

4. The inverter rated current

5. The inverter inductor and its value $\left(L_{i m}\right)$

\section{Filter-Characteristics needed to be considered}

1 The tolerance current $\left(I_{t o l}\right)$

2 The filter capacitor $\left(C_{F}\right)$

3 The damping resistor $\left(R_{\text {Damping }}\right)$

4 Sampling frequency $\left(T_{S}\right)$

5 Delay time $\left(T_{D}\right)$

6 Resonance frequency of the circuit $\left(f_{r}\right)$

\section{Inverter Design}

- Calculate the DC-link voltage of the inverter

$$
r_{D C}>r_{S}^{\prime} \cdot \sqrt{2} \cdot 120{ }^{\prime} i=800 r^{\prime}
$$


- Calculate the voltage ratings of the IGBT switches

$$
V_{B l o c k i n g}>150 \%-200 \% \cdot V_{D C}=1000 \mathrm{~V}-1200 \mathrm{~V}
$$

- Choose the IGBT switch current rating

$$
I_{I G B T}>2 \cdot I_{l d \max }=2 \cdot 50=100 \mathrm{~A}
$$

- First of all, it is required to define the harmonic order and calculate the maximum compensated frequency. Harmonics of the order of 5 to 13 need to be eliminated.

$$
f_{C \text { omp }-\max }=13 \times 50 \mathrm{~Hz}=650 \mathrm{~Hz}
$$

- Calculate the switching frequency

$$
f_{S} \approx(20-40) \cdot f_{\text {Comp-max }}=13-26 \mathrm{kHz}
$$

- The sampling frequency of the inverter is then chosen to be $20 \mathrm{kHz}$. The sampling interval is then

$$
T_{S}=50 \mu \mathrm{sec}
$$

- Decide on the value of the tolerance current. Hysterisis error of $\pm 10 \%$ is to be considered which implies that the value of the total hysterisis band is

$$
I_{\delta}=10 \mathrm{~A}
$$

- The tolerance current of the inverter is calculated from equation (3.9)

$$
I_{\text {tol }} \approx 2.115 \mathrm{~A}
$$

- Calculate the inverter inductance

$$
L_{i m,}>\frac{V_{D C}}{f_{S} \cdot I_{j}}=\frac{800 \mathrm{~V}}{20 \mathrm{kHz} \cdot 10 \mathrm{~A}}=4 \mathrm{mH}
$$

- As a check, the hysterisis band error can be calculated from a different equation. This equation is equivalent to the case of a stalled induction motor. which is the same as the case outlined here at the instants of the zero crossings of the supply voltage $[102]$. 


$$
I_{t o l} \approx 2 \cdot \frac{V_{D C}}{9 \cdot f_{S} \cdot L_{i n v}}=2.2 \mathrm{~A}
$$

This value is similar to the above tolerance current calculated from the equations developed in this chapter.

(Note that in order to keep the switching frequency constant at $20 \mathrm{kHz}$, with lower values of inverter inductances, the hysterisis band selected has to be increased by the same ratio.)

\section{AC-Passive Filter Design Procedure}

- The resonance frequency $f_{r}$ of the circuit diagram of Fig.3.2 (again neglecting $L_{\text {Comp }}$ and $\left.L_{F}\right)$ is:

$$
f_{r} \leq \frac{1}{2 \pi} \cdot \sqrt{\frac{L_{S}+L_{i n v}}{L_{S} \cdot L_{i n v} \cdot C_{F}}} \leq \frac{1}{2 \pi} \cdot \sqrt{\frac{0.470 m H+4 m H}{0.470 m H \cdot 4 m H \cdot 10 \mu F}}=2.5 \mathrm{kHz}
$$

- To avoid resonance, the following assumption can be made with sampling frequency $f_{S}$ :

$$
f_{S} \geq 20 \cdot f_{r}
$$

- The filter capacitor $C_{F}$ should be:

$$
C_{F} \geq 10 \cdot \frac{L_{S}+L_{i m w}}{L_{S} \cdot L_{i n,}} \cdot T_{S}{ }^{2} \geq 10 \cdot \frac{0.470 m H+4 m H}{0.470 m H \cdot 4 m H} \cdot(20 \mu s)^{2} \geq 7 \mu F
$$

- Then, the filter resistance $R_{F}$ can be expressed as:

$$
R_{F}=0.15 \cdot \sqrt{\frac{L_{S} \cdot L_{i n}}{C_{F} \cdot\left(L_{S}+L_{i m}\right)}}=0.15 \cdot \sqrt{\frac{0.470 m H \cdot 4 m H}{10 \mu F \cdot(0.470 m H+4 m H)}}=1.32 \Omega
$$

- The desired value occurs in time intervals with a constant length given by:

$$
T_{S} \leq \frac{2 \pi}{20} \cdot \sqrt{\frac{L_{S} \cdot L_{i m} \cdot C_{F}}{\left(L_{S}+L_{i m v}\right)}} \leq \frac{2 \pi}{20} \cdot \sqrt{\frac{0.470 m H \cdot 4 m H \cdot 10 \mu F}{(0.470 m H+4 m H)}} \leq 19.7 \mu \mathrm{sec}
$$

- The control delay time $T_{D}$ of the switching circuit can be expressed by: 


$$
T_{D} \leq \frac{I_{\delta} \cdot\left(L_{S}+L_{i n v}\right)}{2 \cdot V_{D C}}-T_{S} \leq \frac{10 \mathrm{~A} \cdot(0.470 \mathrm{mH}+4 m \mathrm{H})}{2 \cdot 800 \mathrm{~V}}-19.7 \mu \mathrm{sec} \leq 9.7 \mu \mathrm{sec}
$$

- Choose $L_{2}$ and $C_{\mathrm{F}}$ and consider the emissions of the switching frequency

$C_{F}=1.5 \mu F-10 \mu F, R_{F}=1 \Omega-1.32 \Omega, X_{F}$ neglected.

(for $1 \mathrm{mH}=10 \%$, for $2 \mathrm{mH}=5 \%$, for $4 \mathrm{mH}=2.5 \%$ )

\subsection{Summary}

In this chapter, a new method for power quality improvement was proposed. For this purpose, a typical distribution power system with fundamental and harmonic currents and filter circuit parameters was analysed and described. A different filter circuit configuration provides an alternative approach to the existing ones. The proposed approach can achieve a complete elimination of harmonics.

Due to deregulation of the power supply industry, generation, transmission and distribution will not be in the hands of one company in the future. Therefore, the quality of the supply will become more critical and this alternative method of controlling harmonics generated by non-linear loads provides a good solution for the industry.

Having presented the theoretical analysis of the combination of shunt active power circuit and passive filters, an analytical load model will be described in the following chapter for the proposed system. 


\section{Chapter 4}

\section{Analytical Load Models for Power System}

\section{Components}

\subsection{Introduction}

Modern power systems are very complex and consist of all types of electrical equipment, such as power plants, transmission lines, switch gears, transformers, cables and other consumers. Increasing power consumption by non-linear loads leads to growing power quality problems. Industrial and domestic loads cause harmonics in the network voltages. Large arc-furnaces and two-phase loads (e.g. welding machines) cause voltage fluctuations, voltage imbalance and flicker. These loads need to be modelled correctly for proper analysis of power quality problems.

This chapter deals with the derivation of software models for harmonic compensation and power-factor corrections in low and medium voltage power systems, including distribution transformers and different loads.

For this purpose, each element of the system is discussed and load models are developed. The distribution substations can be supplied by medium or low voltage systems, which require different solutions [150-154]. The results presented in this chapter are based on calculations and measurements according to the IEC regulations. Harmonic loads have greatly increased during the past few years, leading to further distortions of voltage and current waveforms. 
The various techniques proposed for harmonic elimination have mainly discussed power circuit configurations and their control techniques [14,23,33,39,42,48,69]. The process of modelling filter performance in the power system has also contributed to the analysis. However, very few papers analysed the load in order to specifically model it for reactive and/or harmonic compensation.

\subsection{Modelling of the power system}

Fig.4.1 shows a general, medium and low voltage power system with different loads and compensation methods. According to Fig.4.1 the individual network impedances can be calculated and the equivalent circuit for different orders and loads may be given.

Normally, the medium voltage impedance is superimposed on the low voltage power system with a transformer voltage ratio factor $\left(1 / \mathrm{N}^{2}\right)$, whereby the cable capacity must be taken into consideration. The harmonic impedances are mainly determined by the supply transformers, as long as there is no resonance occurring in the power system. The basic data collection is achieved by harmonic measurements on the public power supply system. In the following, a power flow program is developed to analyse the power quality, and all possible system components and loads are discussed.

The network impedance determines the magnitude of the harmonics. Therefore, it is important to find the impedance variations of each load. The models can be divided into four power system groups: residential, industrial, rural and urban, each with 
different load characteristics such as T (transformers), R (resistors), C (capacitors) and $\mathrm{S}$ (series resonance) $[17,21]$. But, these groups cannot be used for the simulation,

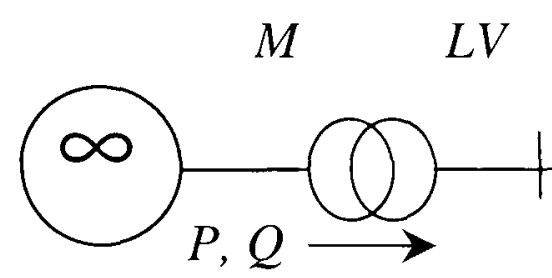

Delivery of Supply

(a)

Consumer

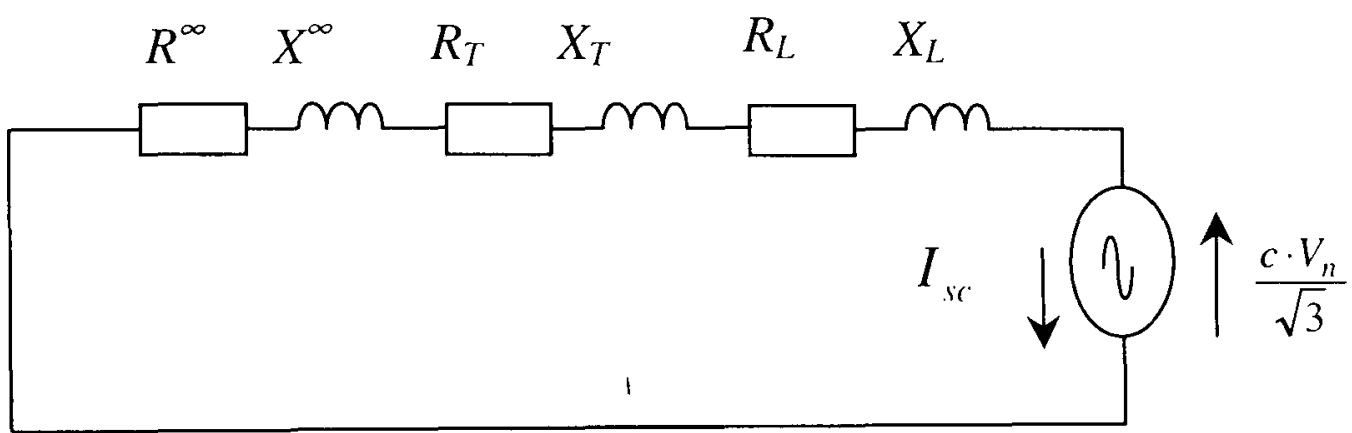

(b)
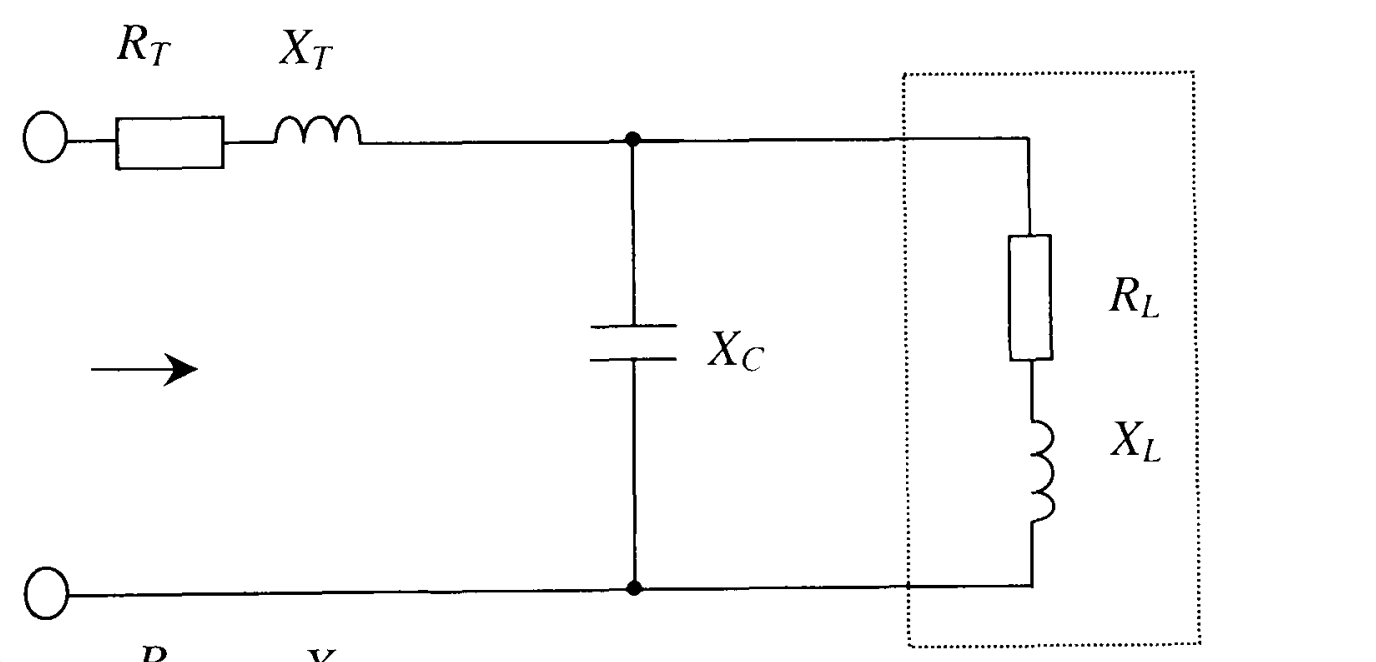

(c)

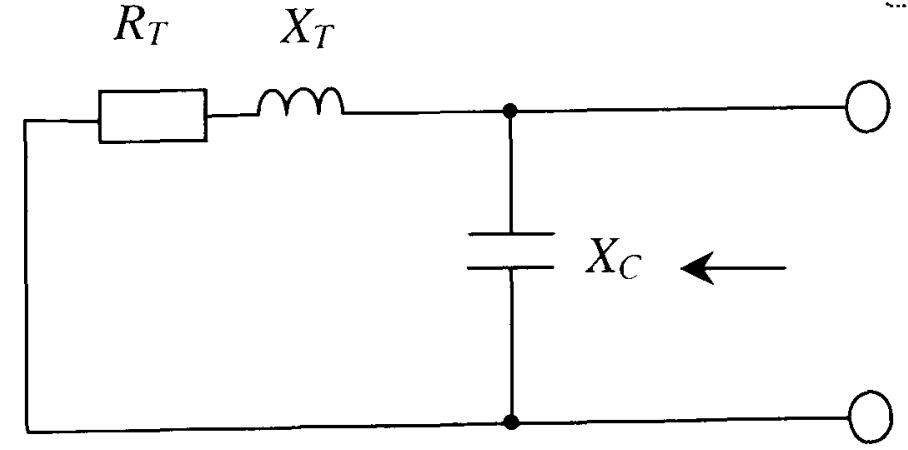

(d)

Fig. 4.1: Structure of a HV, MV and LV-Power System

(a) Single-Line Diagram

(b) Equivalent Circuit for the positive Network

(c) Equivalent Circuit seen from the Transformer Side

(d) Equivalent Circuit seen from the Load Side 
because the simulation program and calculation time would be extremely large. Other attempts have been made to derive statistical load models of the circuits by their probability distribution [35]. As a result, common and frequently occurring loads are considered in this work.

\subsection{Analysis of the characteristics of the power system}

The harmonic current vector $\underline{I}_{h}$ is injected at the point of common coupling (PCC) into the distribution system, producing a voltage drop vector $\underline{V}_{h}$ on the frequency dependent harmonic impedance vector $\underline{Z}_{h}[46,47,56,62-67]$. Therefore, the bus bar impedance can be given as:

$\underline{Z}_{h i}=\frac{\underline{V}_{h i}}{\underline{I}_{h i}}=\left[\underline{Z}_{l d h i} / /\left(\underline{Z}_{T h i}+\frac{\underline{Z}_{M V h i}}{N^{2}}\right)\right]$

\subsubsection{Transformer impedance}

Generally, harmonics are caused by a harmonic current source, transformer and consumer impedance either in series for low voltage systems or in parallel for medium voltage systems. The rated transformer impedance, $Z_{T}{ }^{\text {Rated }}$, can be described by the rated apparent power, $S_{T}{ }^{\text {Rated }}$, transformer percentage short circuit voltage. $v_{s c}{ }^{\text {Rated }}$, and the percentage ohmic voltage drop $v_{R}^{\text {Rated }}$

$\underline{Z}_{T}^{\text {Rated }}=\frac{V_{s}^{2}}{S_{T}^{\text {Rated }}} \cdot\left(v_{R}^{\text {Rated }}+j v_{\text {Stray }}{ }^{\text {Rated }}\right)$ 
$v_{\text {Stray }}{ }^{\text {Rated }}=\sqrt{\left(\left(v_{s i}^{\text {Rated }}\right)^{2}-\left(v_{R}^{\text {Rated }}\right)^{2}\right)}$

where $v_{\text {Stray }}^{\text {Rated }}$ is the percentage stray voltage drop of the transformer.

Taking the harmonics into consideration, then the resistance and inductance of the transformer become [155]

$$
\begin{aligned}
& R_{T h i}=R_{T} \cdot i^{x} \rightarrow x=0.1, \ldots, 0.9 \\
& L_{T_{h i}}=L_{T} \cdot \frac{1-i^{x}}{e^{4} \cdot i} \rightarrow i>1
\end{aligned}
$$

\subsubsection{Ohmic Loads}

Ohmic Loads apply to domestic and industrial categories. The equivalent resistance $R_{l d}$ of the ohmic load can be calculated with the nominal supply voltage $V$, and consumer power $P_{l d}$ (Fig.4.2). It is to be noted that $R_{T h}$ and $X_{T h}$ connected in parallel with the load and supply represent the distribution transformer.

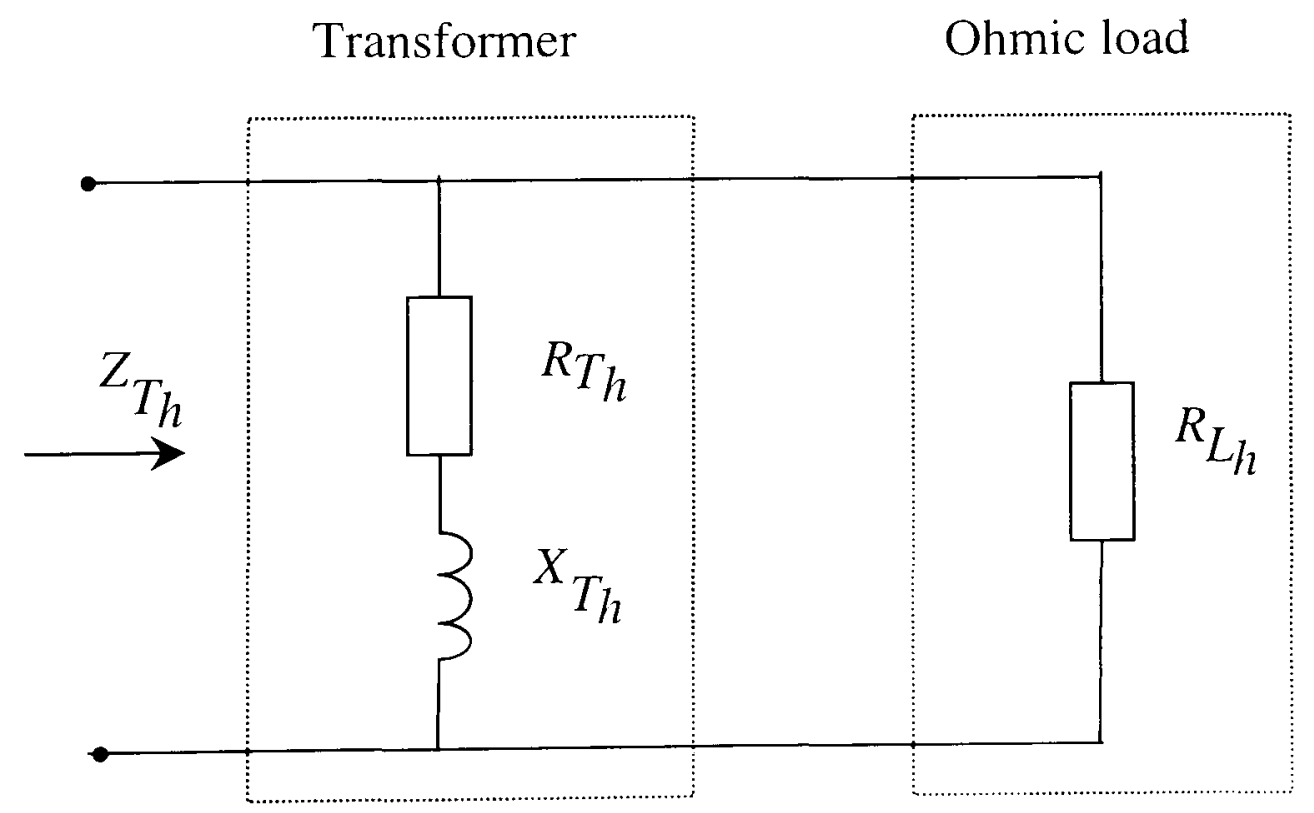

Fig.4.2: Equivalent circuit of ohmic load 


$$
R_{l d}=\frac{V_{s}^{2}}{P_{l d}}
$$

\subsubsection{Ohmic and inductive load (Motor)}

The impedance of an asynchronous motor can be represented by the rated motor

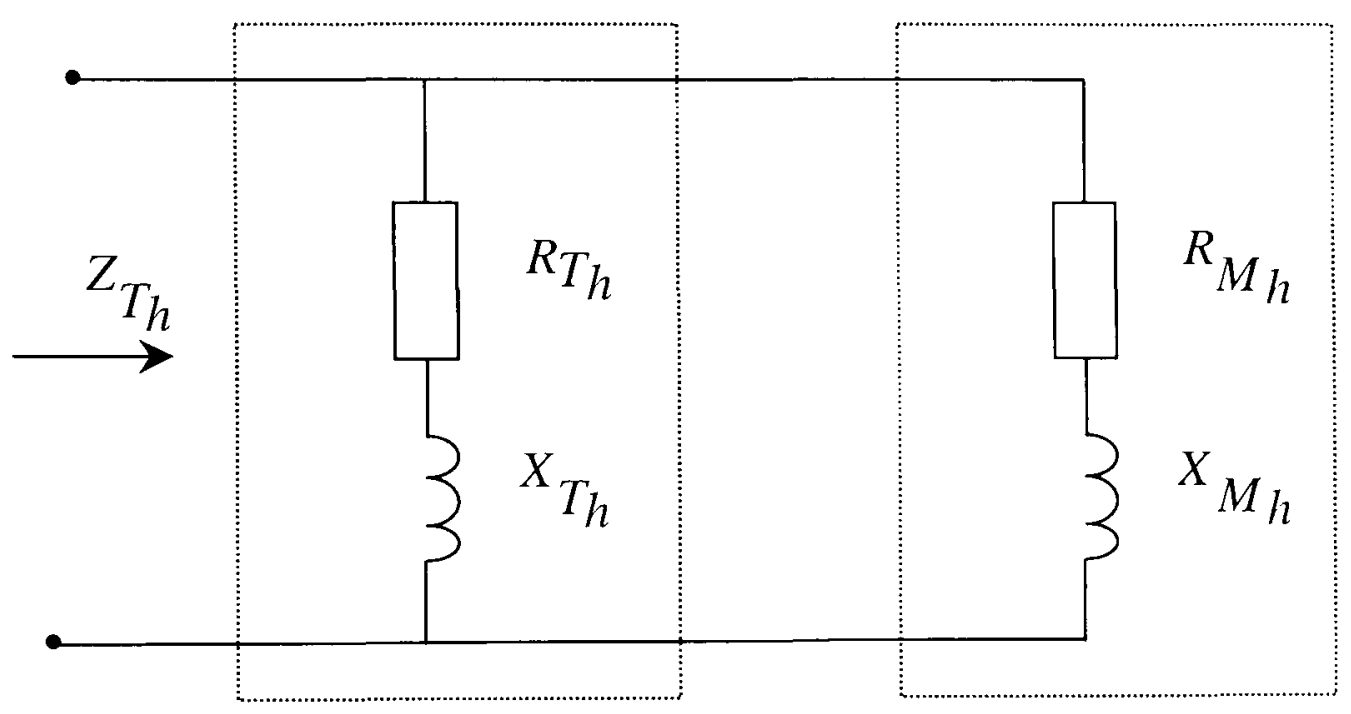

Fig.4.3: Equivalent circuit of ohmic and inductive load

current and its starting current ratio $\frac{I_{M}{ }^{\text {Rated }}}{I_{\text {Starting }}}$ and the motor rated power $S_{M}{ }^{\text {Rated }}$

(Fig.4.3).

$\underline{Z}_{M}^{\text {Rated }}=\frac{V_{s}^{2}}{S_{M}{ }^{\text {Rated }}} \cdot \frac{I_{M}^{\text {Rated }}}{I_{\text {Starting }}} \cdot\left(\cos \varphi_{M}^{\text {Rated }}+j \sin \varphi_{M}^{\text {Rated }}\right)$

where $\cos \varphi_{M}{ }^{\text {Rated }}$ is given on the name plate of the motor. 


\subsubsection{Ohmic, inductive and capacitive load (Capacitor)}

Power systems and facilities contain capacitors which affect the network impedance in such a way that the resonance frequency, $f_{r}$, in the system can be estimated as for the case of Fig.4.4, by neglecting the motor impedance for low voltage systems.

$$
f_{r}=\frac{1}{2 \pi \cdot \sqrt{L_{T !} C_{l d}}}
$$

Certain terms in Eq.4.8 can be re-written as follows:

- Inductance $L_{T_{1}}$ can be represented as a function of the short circuit power in the system $S_{s c}{ }^{\infty}$ (neglecting resistors)

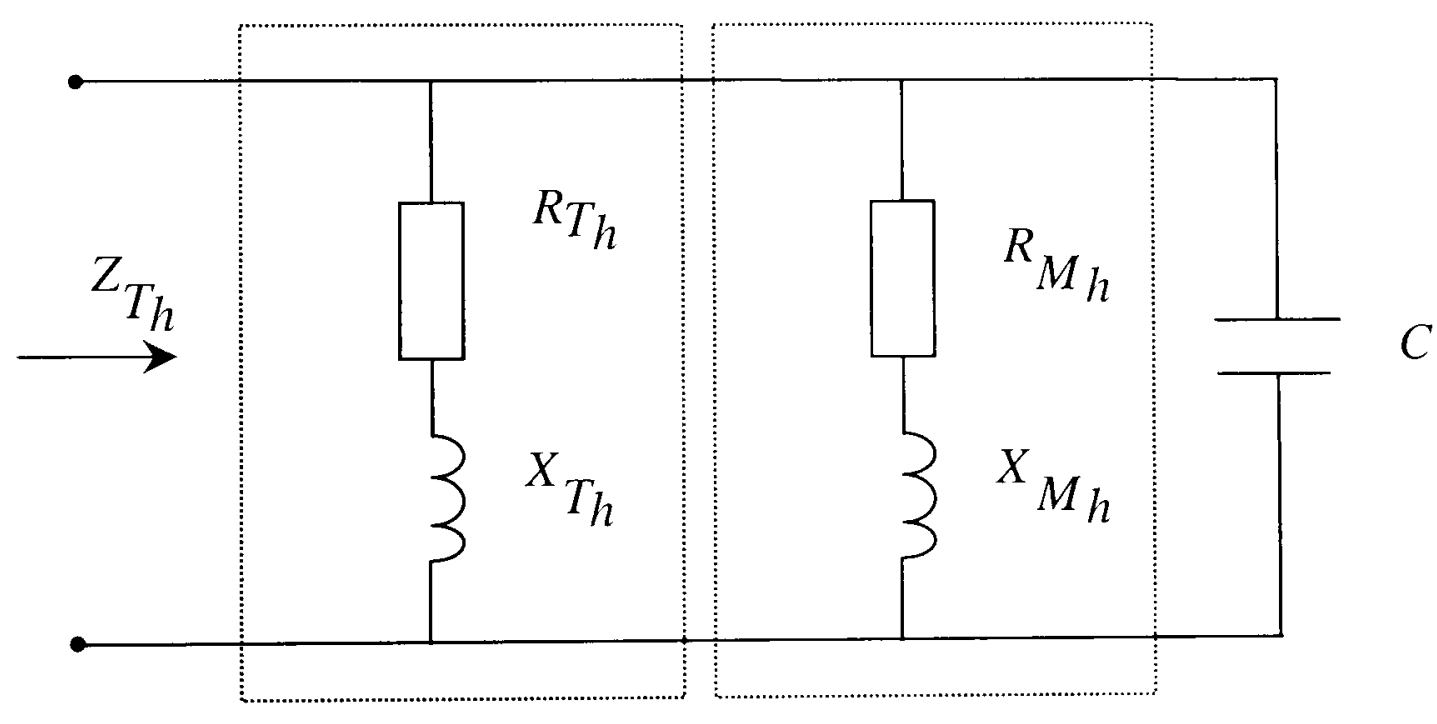

Fig.4.4: Equivalent circuit of ohmic, inductive and capacitive load

$$
L_{T_{1}}=\frac{V_{s}^{2}}{\omega_{1} S_{s i}^{\infty}} .
$$

- Then the capacitor $C_{l d}$ can be calculated from the capacitive charging power $Q_{C}$. obtained from the difference between the actual and required reactive power of the system. 


$$
C_{l d}=\frac{Q_{C}}{\omega_{1} V_{s}^{2}}
$$

- The ratio of the resonance frequency, $f_{r}$, to the fundamental frequency. $f_{1}$. is given by:

$$
\frac{f_{r}}{f_{1}} \approx \sqrt{\frac{S_{s c}^{\infty}}{Q_{C}}}
$$

\subsubsection{Medium voltage power system}

The occurrence of resonance in medium voltage power systems is high and all consumers (loads, capacitances) must be taken into account. The resonance in a medium voltage system can be represented, as in Fig.4.5, with the short-circuit parameters at the PCC.

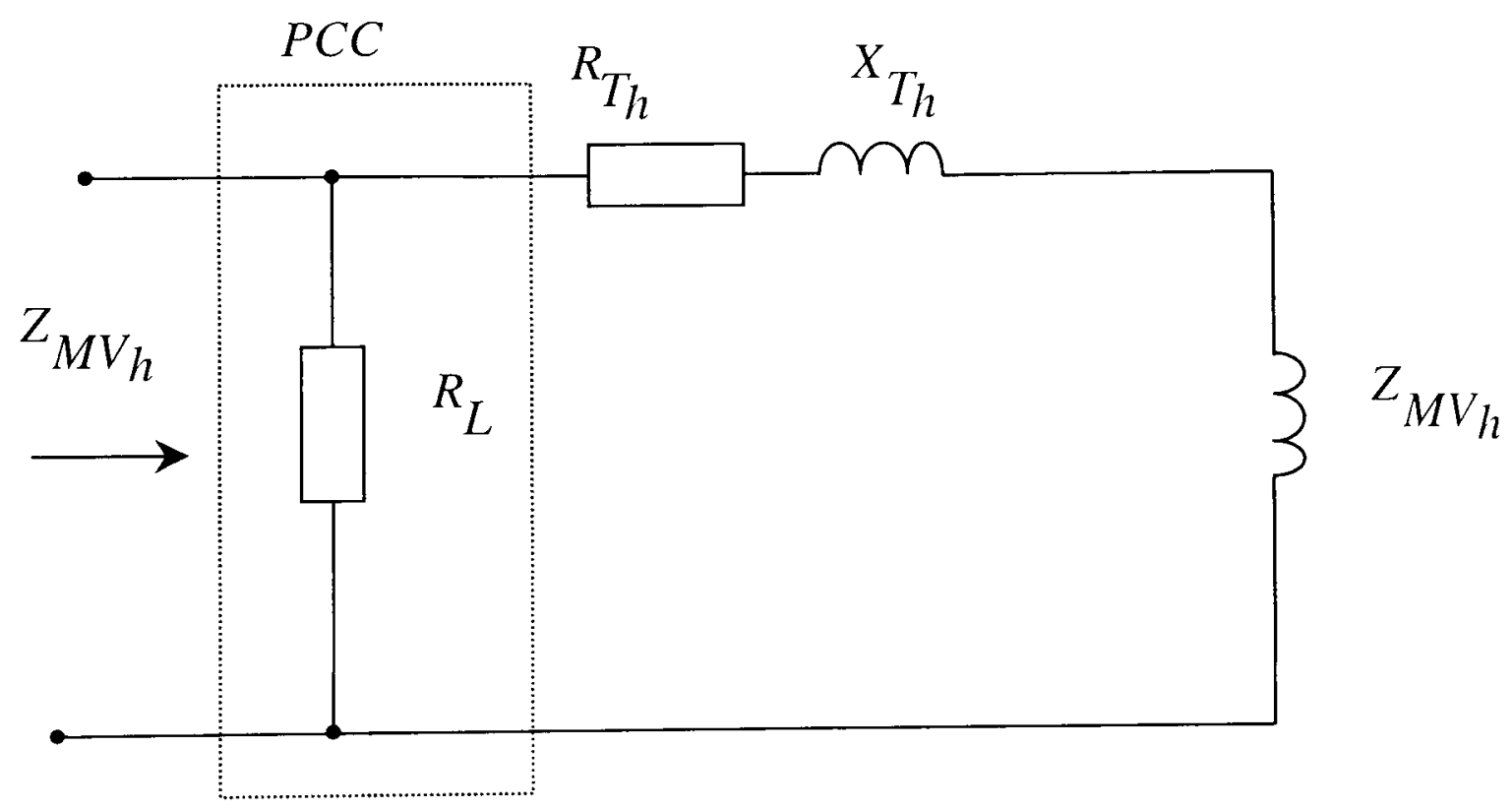

Fig.4.5: Equivalent circuit of medium voltage power system

$\frac{f_{r}}{f_{1}} \approx \sqrt{\frac{S_{w}^{\infty}}{Q_{c}}}=\frac{1}{V_{s}} \cdot \sqrt{\frac{\sqrt{3} V_{s} I_{s c}}{\omega_{1} C_{l d}}}=\sqrt{\frac{\sqrt{3} I_{s}}{I_{C l d}}}$ 
where, the value of $I_{C l d}$ is calculated for the current of the capacitive load on the referred low voltage side.

Furthermore, the total short circuit power in Eq.4.12 is the sum of the medium (MV) and low (LV) voltage powers and is given by

$S_{s c}^{\infty, t o t}=\sum S_{s c_{M V}}+\sum S_{s c_{L V}}$

\subsubsection{Losses in the power system}

The feeder losses due to harmonic currents are added to the losses caused by real and reactive currents to give the total power losses in the feeder system.

$$
P_{\text {loss }}=R \cdot\left(I_{\text {real }}^{2}+I_{\text {reactive }}^{2}+I_{\text {harmonics }}^{2}\right)
$$

\subsubsection{Earth leakage}

It is very important to distinguish the earth remaining current $I_{r e m}$ from the earth fault current, $I_{C E}$, which defines the value of the allowable earthing resistance, $R_{E}$. The earth remaining current, $I_{r e m}$, can be calculated from the equation [12]

$$
\frac{I_{r e m}}{I_{C_{E}}}=\sqrt{\left(\frac{I_{r e m 1}}{I_{C_{E}}}\right)^{2}+\sum_{i}\left(\frac{I_{i}}{I_{C_{E}}}\right)^{2}}
$$

In industry, capacitors and absorption circuits (filters) are installed to limit harmonics, which influence the network impedance. The characteristics of these filters can be evaluated as follows:

\section{- Capacitor $\mathbf{C}$}

$$
C_{F}=\frac{Q_{i}}{\omega_{1} V_{s}^{2}}
$$




\section{- Inductor $\mathbf{L}$}

$$
L_{F}=\frac{1}{\omega_{1}{ }^{2} C_{F}}=\frac{V_{s}^{2}}{\omega_{1} \cdot Q_{C}}
$$

\section{- Resistance R}

$$
R_{F}=\frac{2 \pi \cdot f_{1} \cdot L_{F}}{Q_{C}}=\frac{V_{s}^{2}}{Q_{C}{ }^{2}}
$$

\subsection{Proposed Load Model for Power System Components}

It is evident that the load model must be constructed according to the type of loads in the low and medium voltage system. For each load, there exists only one model which can be put together to build up the final load model. Fig 4.6 shows a single-phase load model with the frequency dependent network impedance and harmonic sources.

In order to give the best model, some assumptions must be made:

1. The cable length in the low voltage system is not too long.

2. There are not too many points of power connection (radial systems).

3. Power supply and loads are known.

\subsection{Simulation Results of the Proposed Model}

The valid compatibility level for particular harmonic voltages in public low voltage (LV) and medium voltage (MV) systems and the measured values are given in Table.4.1. These values were introduced into the load harmonic simulation model (Fig. 4.7) to investigate the power system performance. Fig. 4.8 shows a comparison between the compatibility levels with the calculated harmonics. 


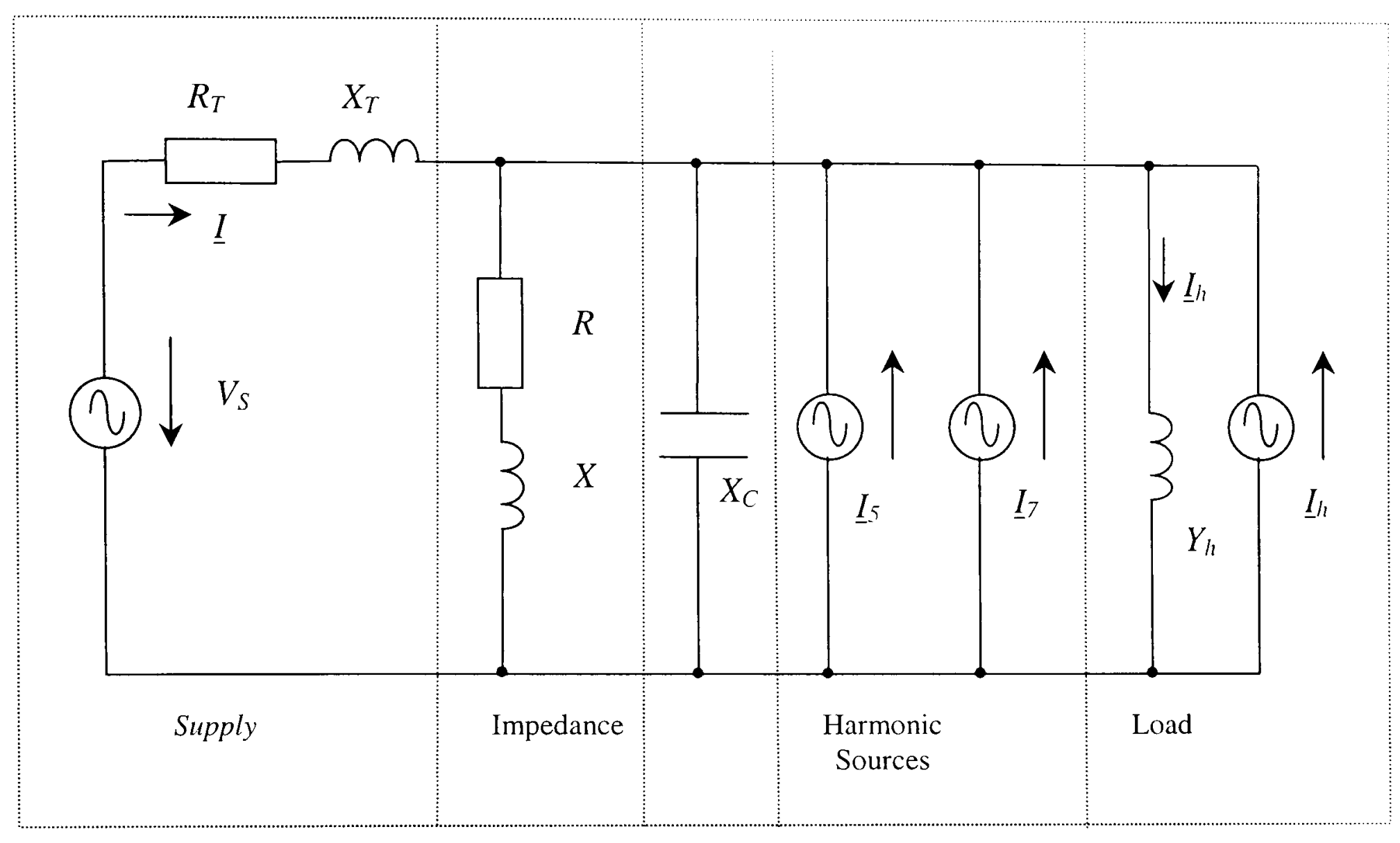

Fig.4.6: Equivalent circuit of different harmonic orders and loads

Table.4.1: Valid Compatibility Level (IEC 1000-2-2) and Measured Values in Industrial Power Systems

\begin{tabular}{|c|c|c|c|}
\hline Harmonic & Harmonic & measured values & measured values \\
Order $v$ & Voltage in \% & LV & MV \\
\hline $\mathbf{3}$ & IEC 1000-2-2 & voltage in \% & voltage in \% \\
\hline $\mathbf{5}$ & 5.0 & 0.54 & 0.54 \\
$\mathbf{7}$ & 5.0 & 5.87 & 5.75 \\
$\mathbf{9}$ & 1.5 & 1.55 & 1.35 \\
$\mathbf{1 1}$ & 3.5 & 0.47 & 0.18 \\
\hline $\mathbf{1 3}$ & 3 & 0.37 & 0.27 \\
\hline
\end{tabular}




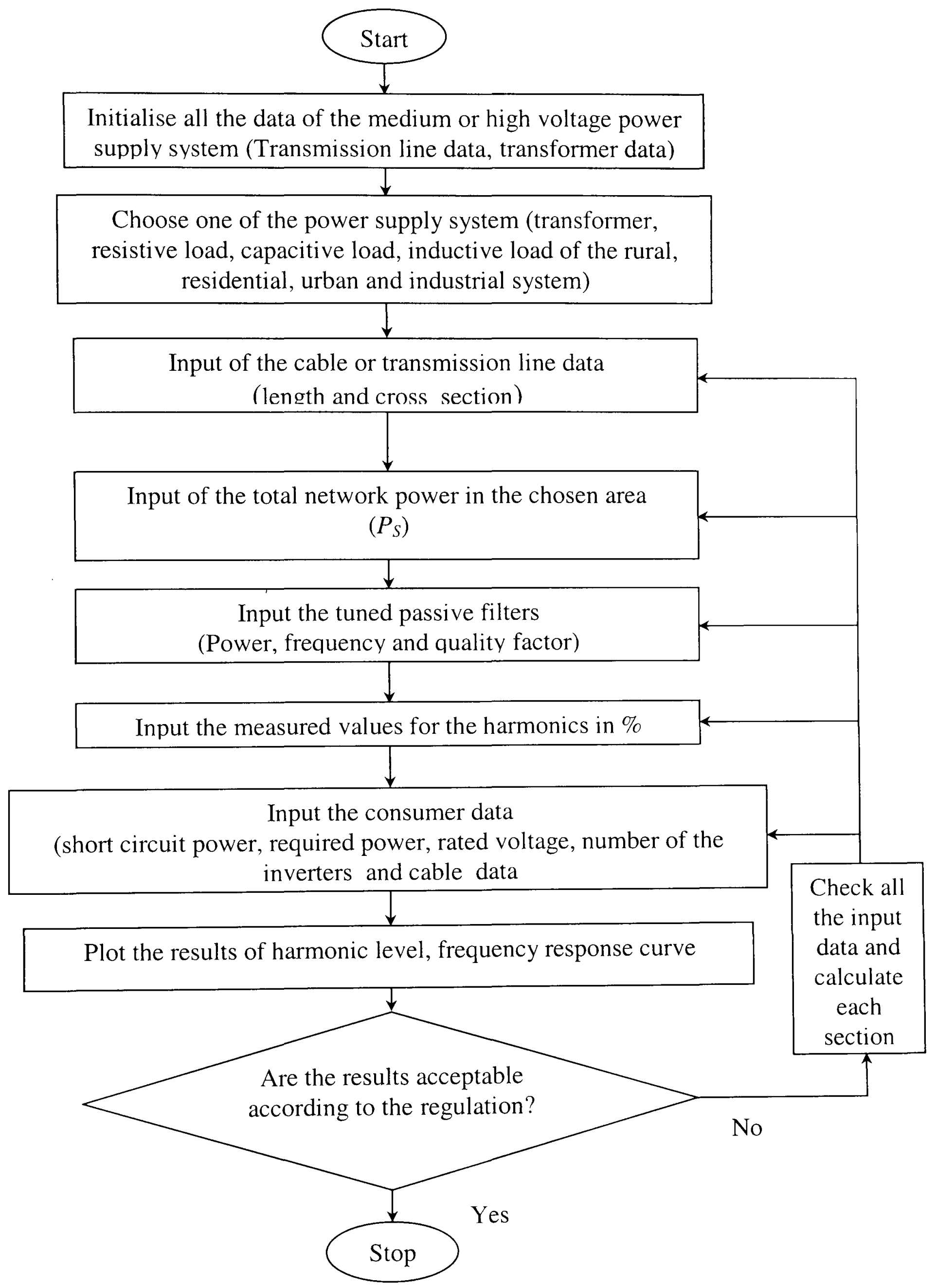

Fig.4.7: Flow chart for the calculation and simulation of harmonics in the power system 


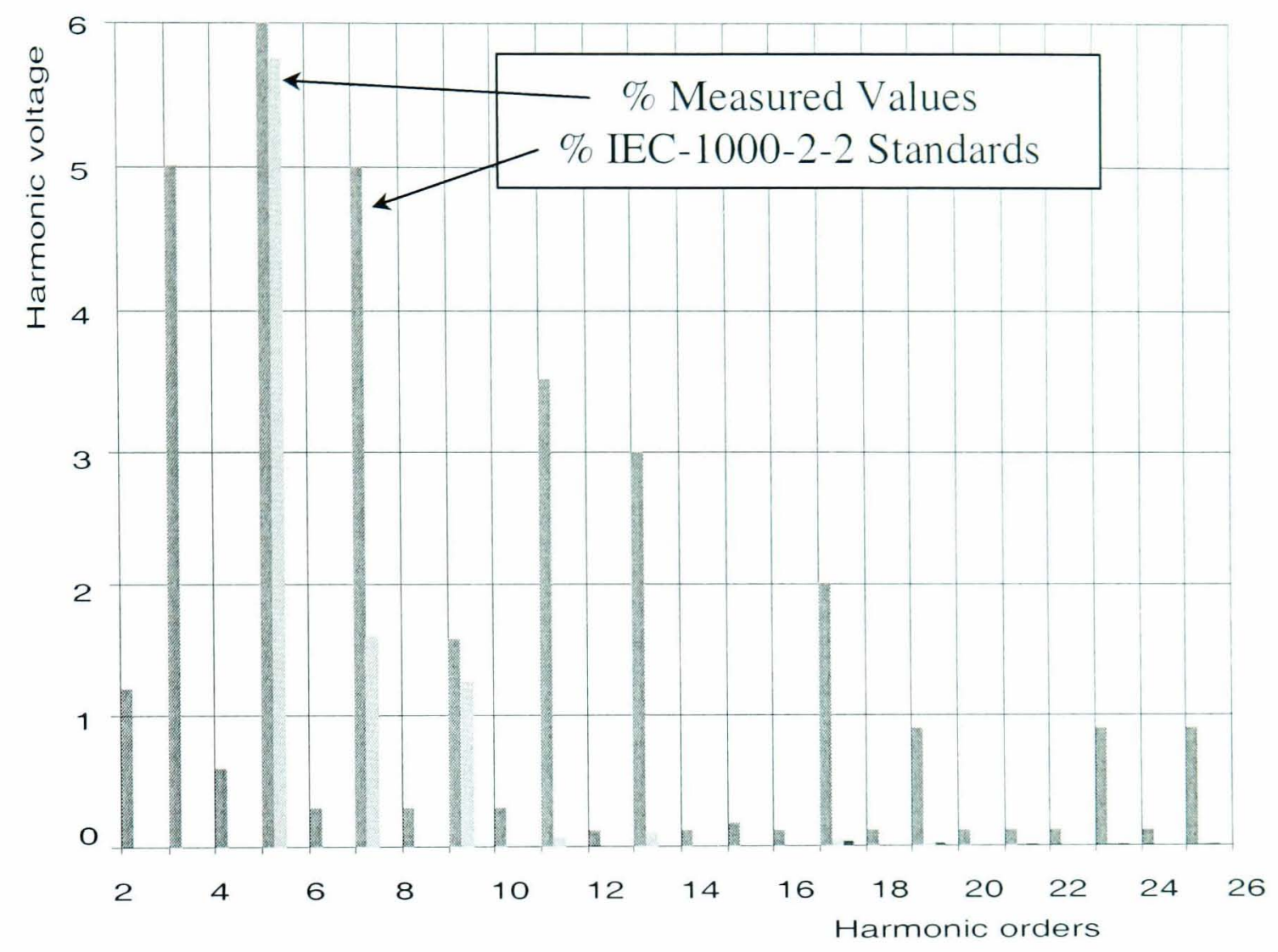

Fig. 4.8: Comparison of the harmonic values

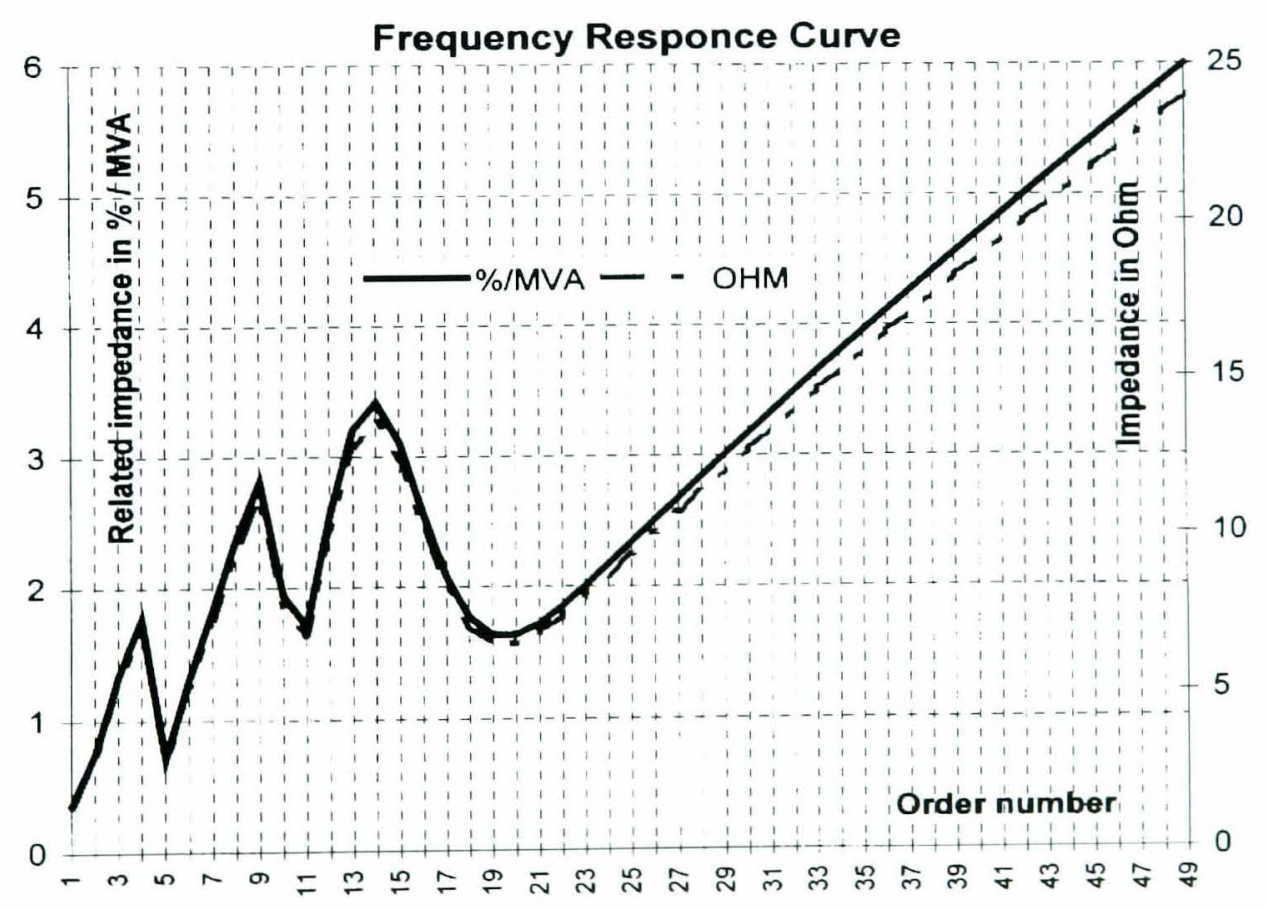

Fig. 4.9: Simulation result 
Fig.4.9 shows the simulated curve of network impedance and efficiency of load modelling. The accuracy of this model has been checked up to $110 \mathrm{kV}$ at $2 x+0 \mathrm{MVA}$ transformer and 8000 MVA network short circuit power.

\subsection{Discussion of the Proposed Model}

In reality, it is extremely difficult to model very complicated power distribution systems and to measure the frequency dependent network impedance, which affects the values of the components [156-158].

The information presented in this chapter aims to give a better understanding of the harmonics in a power system using a computer program to calculate harmonic power flow. A large medium and low voltage distribution power system was selected to demonstrate the application of this program. The harmonic current sources were investigated by measuring harmonics on the low and medium voltage side. The network impedances were determined and calculated based on the measurements at the point of common coupling (PCC).

This method can be used to estimate the response behaviour of the power system in conjunction with a frequency range, which is dependent on the network type, day and time of year. Furthermore, the expected harmonics and reactions on the mains can be forecasted and evaluated to define the electromagnetic compatibility and emission limits of the different loads.

Regarding derivation of the distribution loads, the following statements can be made: 
1. Transformers and feeders should be represented by equivalent elements.

2. Cable and lines should be represented by equivalent $\Pi$-models.

3. Other elements such as resistance, inductance and capacitance should be modelled by their actual system configuration.

4. Induction motors should be modelled by skin effect in the stator and rotor and by apparent slip at the superimposed frequency.

\subsection{Summary}

This chapter has presented different load models of the power system components. For a better understanding of the harmonics and their levels, a software program has been developed. In the next two chapters, the software simulation and hardware implementation of the proposed circuit will be respectively discussed. 


\section{Chapter 5}

\section{Simulation Results}

\subsection{Introduction}

The compensation system proposed in chapter 3 with all its theoretical equations as well as the load modelling outlined in chapter 4 needs to be simulated before implementing it in a laboratory prototype. The simulation of the operating conditions of the system is presented in this chapter. These include several aspects of the system such as loss calculations and the effect of nonlinearities in the load models. Real-time data recorded from power systems are implemented inside the dedicated software programs written for the simulation purposes. The simulation results are presented during the course of this chapter.

\subsection{Losses in the Proposed System}

The switching devices chosen for this circuit in the simulation process are critical from the point of view of loss calculations. IGBTs are chosen to implement the system prototype for reasons explained in the following chapter. The simulation of the system should include the calculation of the losses in the switches due to the switching frequency employed as well as the case of the conduction losses as is explained in this section.

IGBTs and diodes operate predominantly in switching modes in which there are different static and dynamic conditions. The conduction and switching losses oceur in 
all these conditions, which leads to a rise in temperature. Fig. 5.1 gives an overview

of the losses in IGBTs as well as losses in other components of the proposed system.

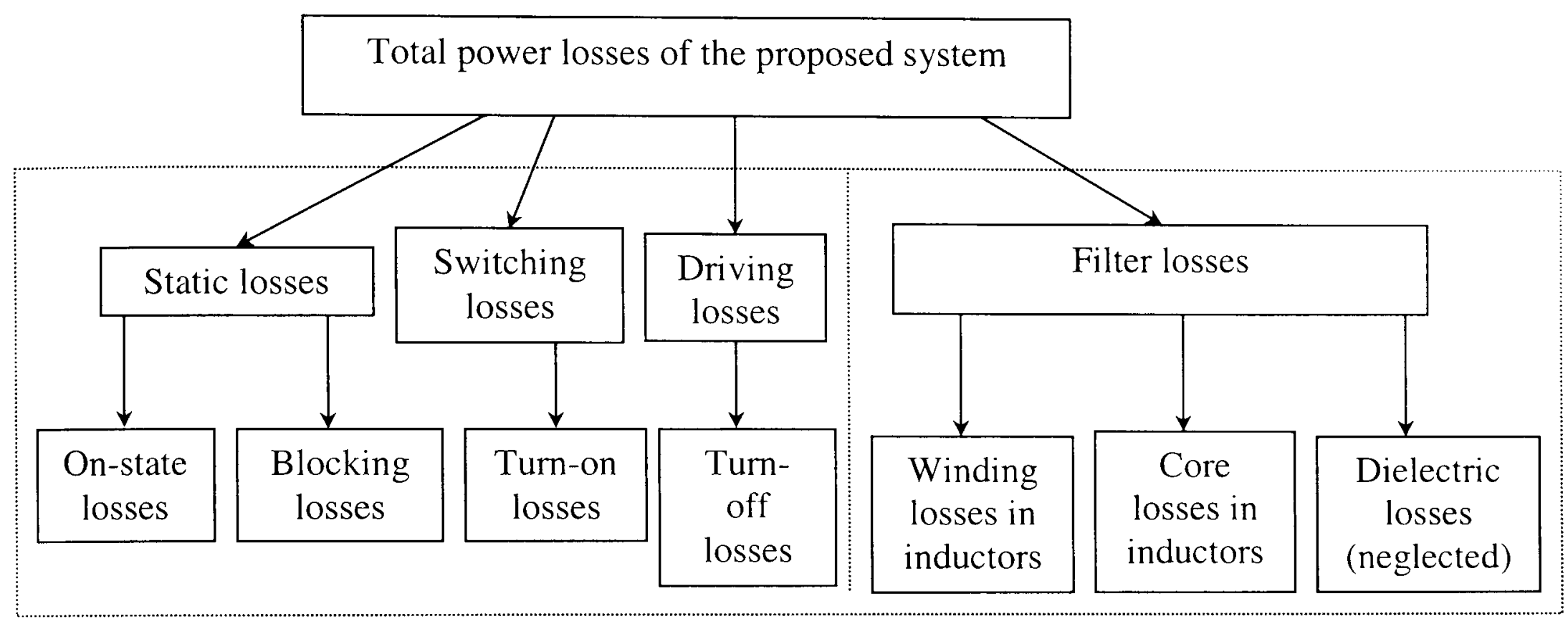

Fig. 5.1: Detailed power loss subdivision of the proposed

\section{i. Load current}

\subsubsection{Device Losses}

To calculate the device losses, the operating curves are put into the program (Fig.5.2).

Then, the switching losses for the turn-on and turn-off waveforms of an IGBT can be given as [159]:

$$
\begin{gathered}
E_{I G B T-o n}=\int_{0}^{t_{r i}+t_{d}+t_{b}} i_{C o l} \cdot V_{C E} \cdot d t \\
E_{I G B T-o f f}=\int_{-t_{d}}^{t_{f}} i_{C o l} \cdot V_{C E} \cdot d t
\end{gathered}
$$

and

$P_{s}=f_{S} \cdot\left(E_{l G B T-o n}+E_{l G B T-o f f}\right)$ 
The conduction losses can be calculated using the conduction state voltage $V_{F}$. onstate slope resistance $r_{F}$ and the periodic device current:

$$
P_{C} \approx \frac{1}{T} \int_{0}^{T}\left(V_{F}+r_{F} \cdot i_{D / I G B T}\right) \cdot i_{D / I G B T} \cdot d t
$$

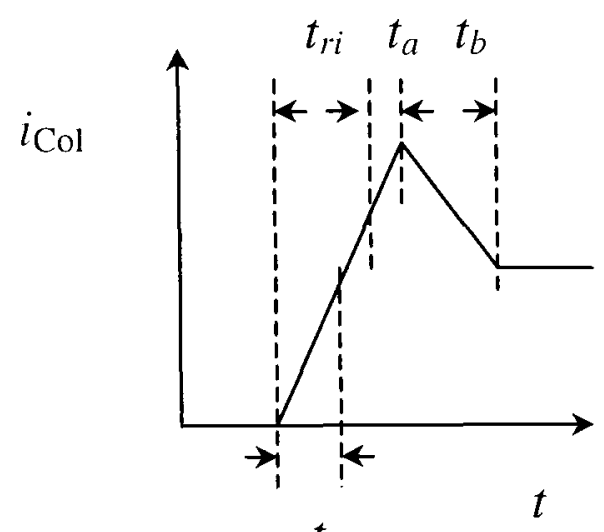

$t_{0}$

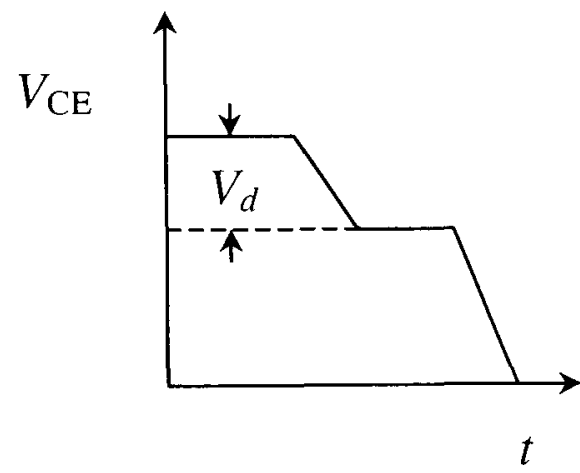

(a)
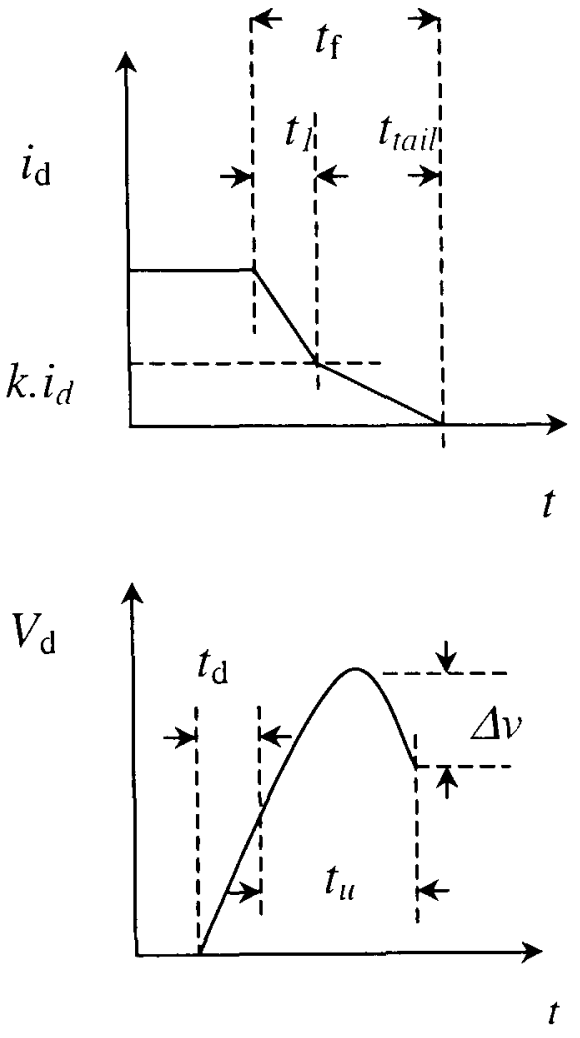

(b)

Fig. 5.2: (a) Turn-on and (b) Turn-off waveforms

\subsubsection{Filter Losses}

The filter inductors can be given by the winding losses $P_{C u}$ and core losses which are discussed as follows:

$$
P_{C u}=R_{L} \cdot i^{2} R M S
$$

The resistance $R_{L}$ is very dependent on skin and proximity effects. The core losses can be calculated as:

$$
P_{\text {rim }}=V o l_{c} \cdot p_{0} \cdot f_{S}^{E_{F}} \cdot B_{\text {s. }}^{e_{B} B}
$$


where

$i_{R M S} \quad$ Inductor current

Vol $\quad$ Magnetic volume

$f_{S} \quad$ Switching frequency

$p_{0}, e_{F}, e_{B} \quad$ Material-dependent constants

$B_{A C, s} \quad$ Flux density of the AC-component at the switching frequency

The filter capacitor losses can be determined from the resistive and dielectric losses.

\subsection{Simulation of the Proposed Inverter Power Circuit}

Inverter and filter characteristics are given in Appendix B. Simulation program is shown in Appendix C. Simulations of the overall proposed system take the following steps:

\subsubsection{Simulation of the losses}

Fig.5.3 shows the investigated single-phase system. Calculation is performed before and after the filter circuit to find an optimum filter design and to minimise the power losses in the circuit. The results of the total losses are showed in Table 5.1.

The total losses for the following set of rating in IGBTS can be calculated approximately from the relation:

$P_{T} \approx(2$ to 3$) \cdot\left(P_{\text {conduction }}+P_{\text {switching }}\right)$ 


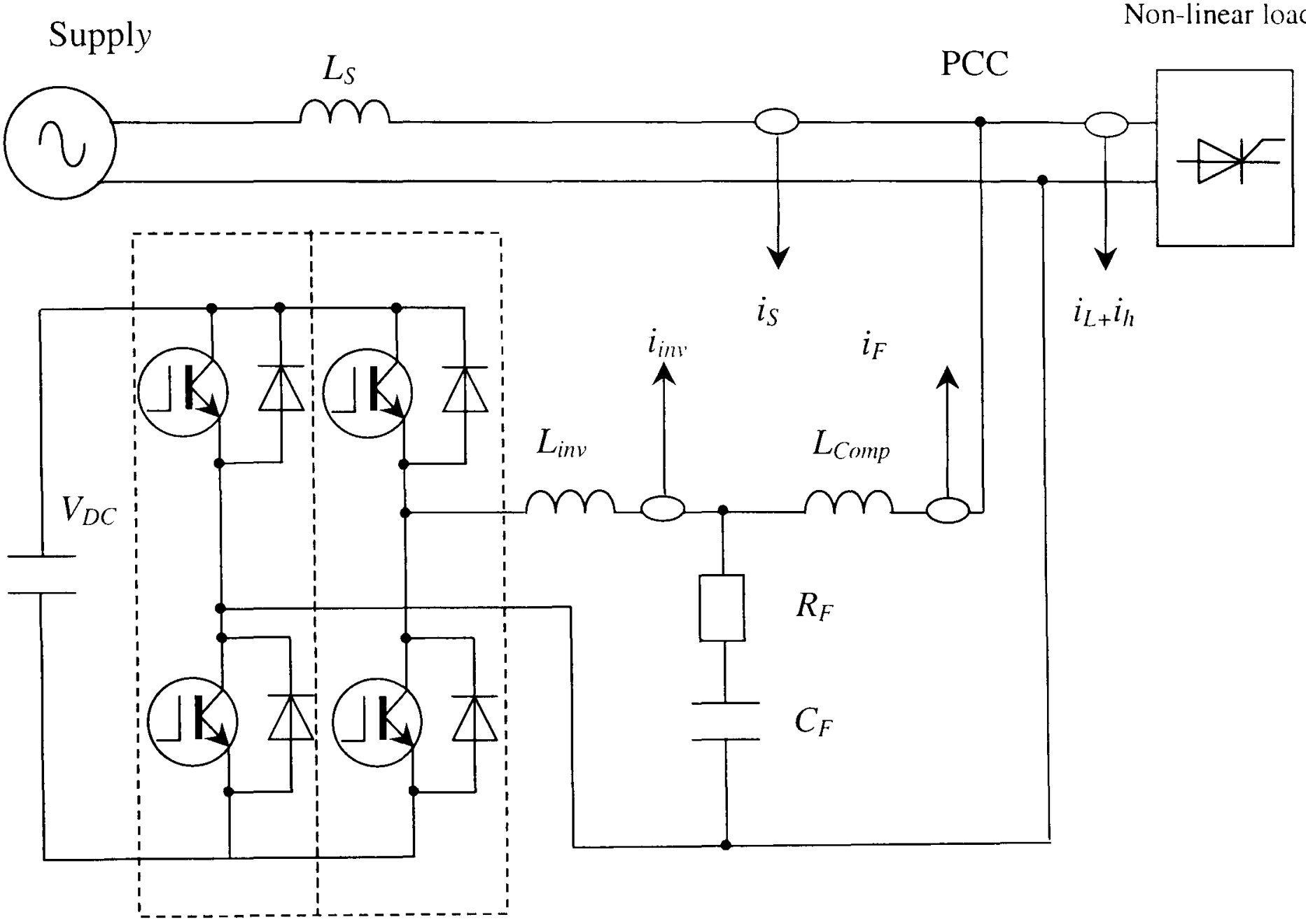

Fig. 5.3: Single-phase experimental set-up

Table 5.1 : Total losses of the circuit

\begin{tabular}{|l|c|c|}
\hline & Case 1 & Case 2 \\
\hline$i_{r m s}$ & $20 \mathrm{~A}$ & $50 \mathrm{~A}$ \\
\hline$R_{F}$ & $2,5 \mathrm{~W}$ & $3 \mathrm{~W}$ \\
\hline$L_{\text {inn }}$ & $250 \mathrm{~W}$ & $367 \mathrm{~W}$ \\
\hline$P_{\text {conducting }}$ & $61 \mathrm{~W}$ & $205 \mathrm{~W}$ \\
\hline$P_{\text {switching }}$ & $43 \mathrm{~W}$ & $48 \mathrm{~W}$ \\
\hline$P_{\text {total }}$ & $356.5 \mathrm{~W}$ & $623 \mathrm{~W}$ \\
\hline
\end{tabular}


The following steps can be undertaken to reduce the losses:

\section{a) in the inverter circuit}

1. Lower switching frequencies

2. Lower temperature-coefficient heat-sinks

3. Shorter switching times i.e lower gate resistance

\section{b) in the filter circuit}

1. Larger copper cross section

2. Lower flux density in the magnetic core

3. Lower current ripple

As it can be seen from the Table 5.1, the main power losses occur in the semiconductor switches and then in the inductor $L_{i n v}$. It also shows that the passive filter increases the total losses of the circuit which must be determined by optimising the overall circuit. All these calculations were performed by the software program in Appendix A.

\subsubsection{PWM and Hysterisis Switching Techniques}

The clocked hysteris technique [102] and the PWM switching technique used to operate the inverter was introduced in chapter 3 and are analysed and simulated in this chapter.

The PWM-hysterisis controller operates in this case as shown in the block diagram of Fig.5.4. where the error signal is fed to the hysterisis controller. The output of the 
hysterisis controller drives the output stages of the controller. The feedback signal for the hysterisis controller is taken from the output signal of the hysterisis block through an integrator with a time constant approximately equal to the time constant of the overall system. The integrated value is then considered to be the feedback signal which when subtracted from the reference current generates the error driving signal for the hysterisis block. The internal feedback ensures that the maximum switching frequency of the hysterisis controller is determined by the system feedback time constant and is always within the desired range. The clocking of the PWM signal generated by the controller ensures that the switching of the IGBT switches is uniform.

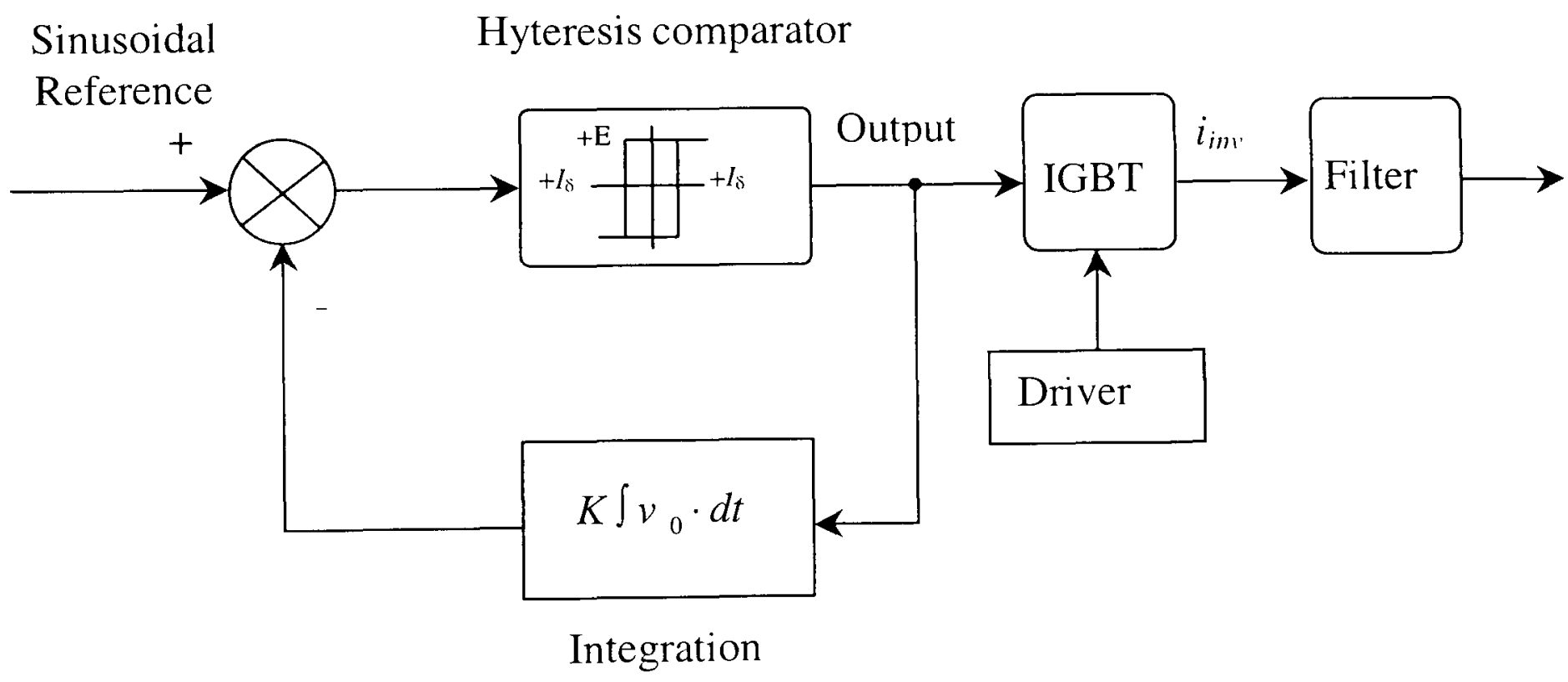

Fig. 5.4: Hysterisis controller operation

The output of the hysterisis controller drives an interlocking circuit, which then decides on which switch to turn on or off, according to the switching modes defined in Table 3.1. 
On the other hand, the ordinary clocked hysterisis controller operates as specified in [102]. It relies on the fact that the feedback signal originates from the actual value of the controlled variable (inverter current). The resulting switching pattern for the switches in the case of a plain hysterisis controller are shown in Fig.5.5, with the corresponding harmonic spectrum of the supply current after compensation shown in Fig.5.6.

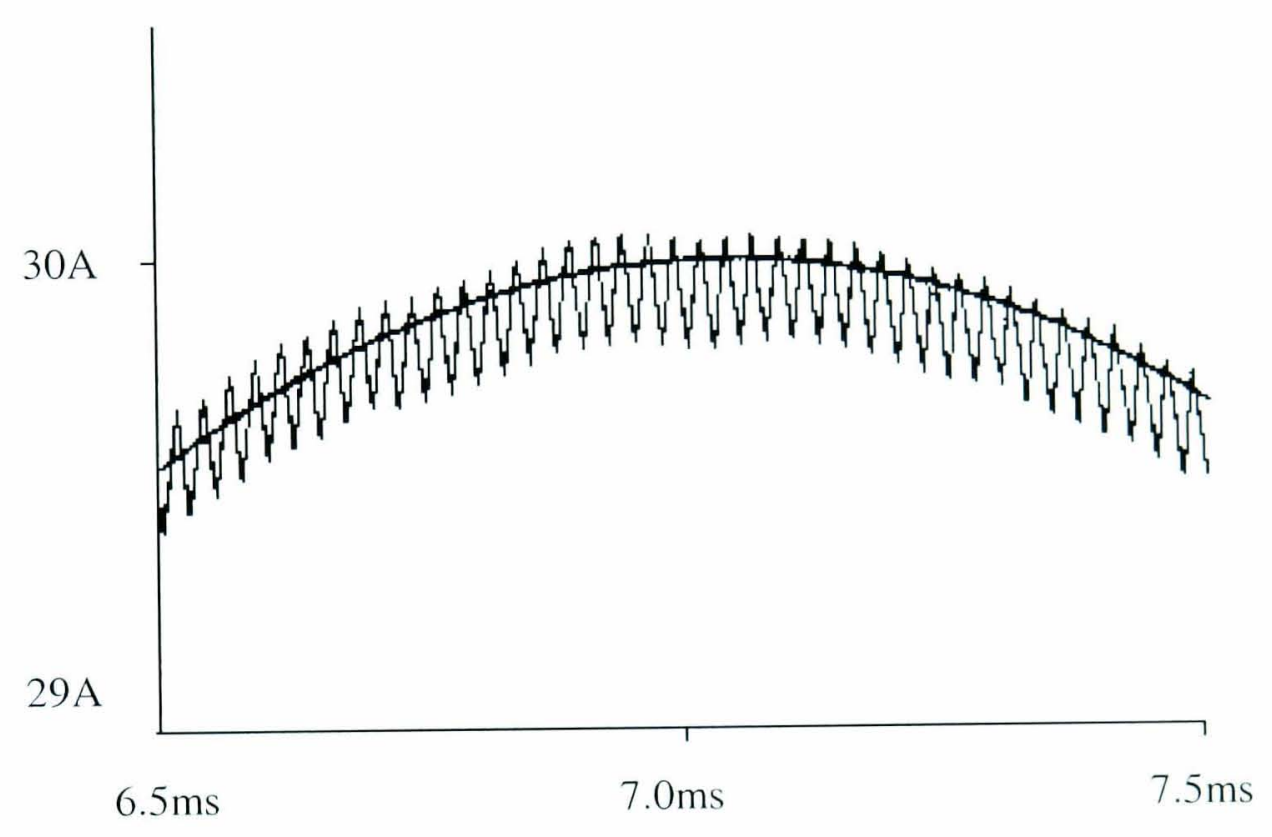

Fig. 5.5: Simulation results of current control of using hysterisis technique

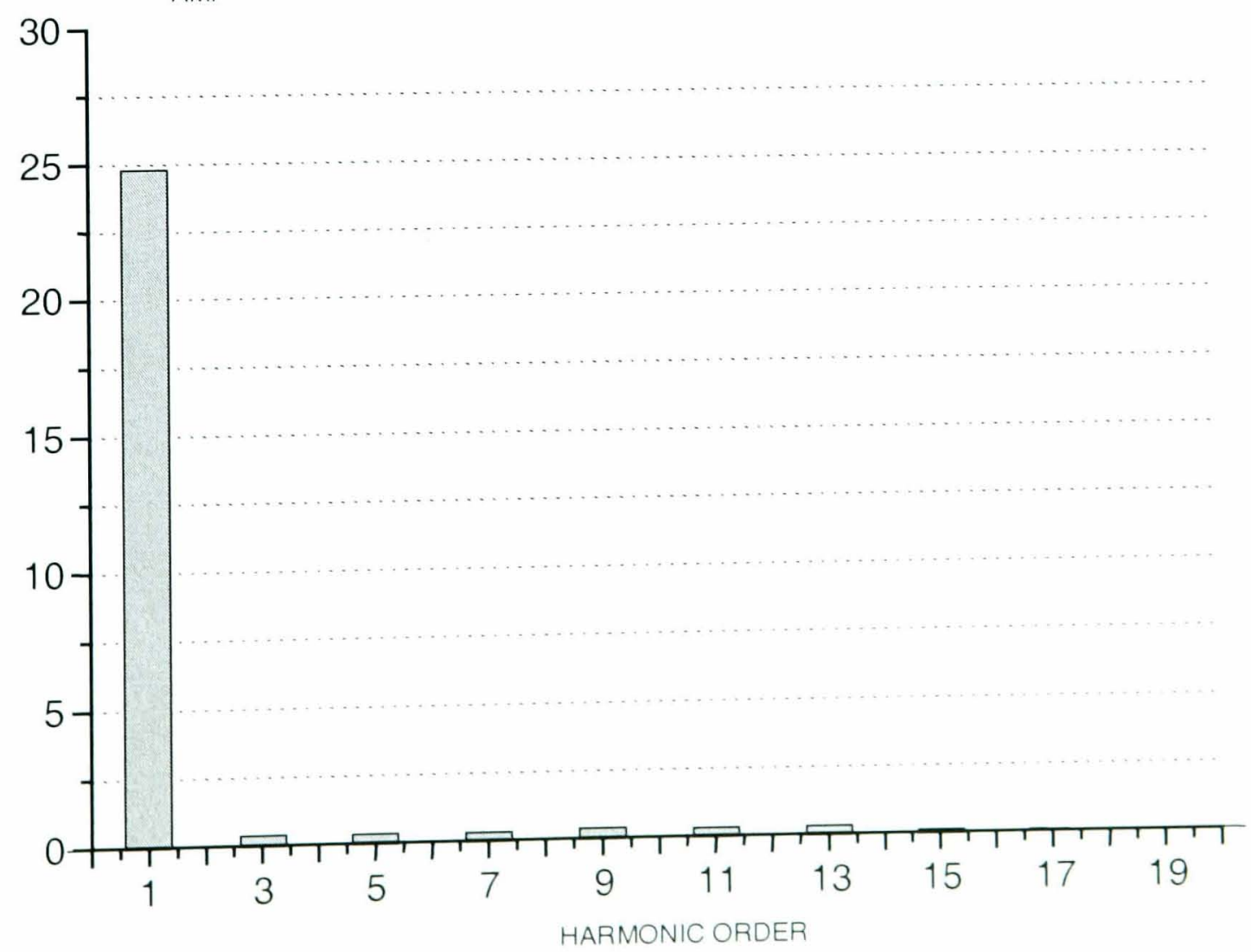

Fig.5.6: Load harmonic content after compensation with hysterisis techniques 
The PWM-hysterisis waveforms are applied to the IGBT switches in the simulated circuit and is shown in fig5.7, with the harmonic spectrum of the resulting supply current shown in Fig.5.8.

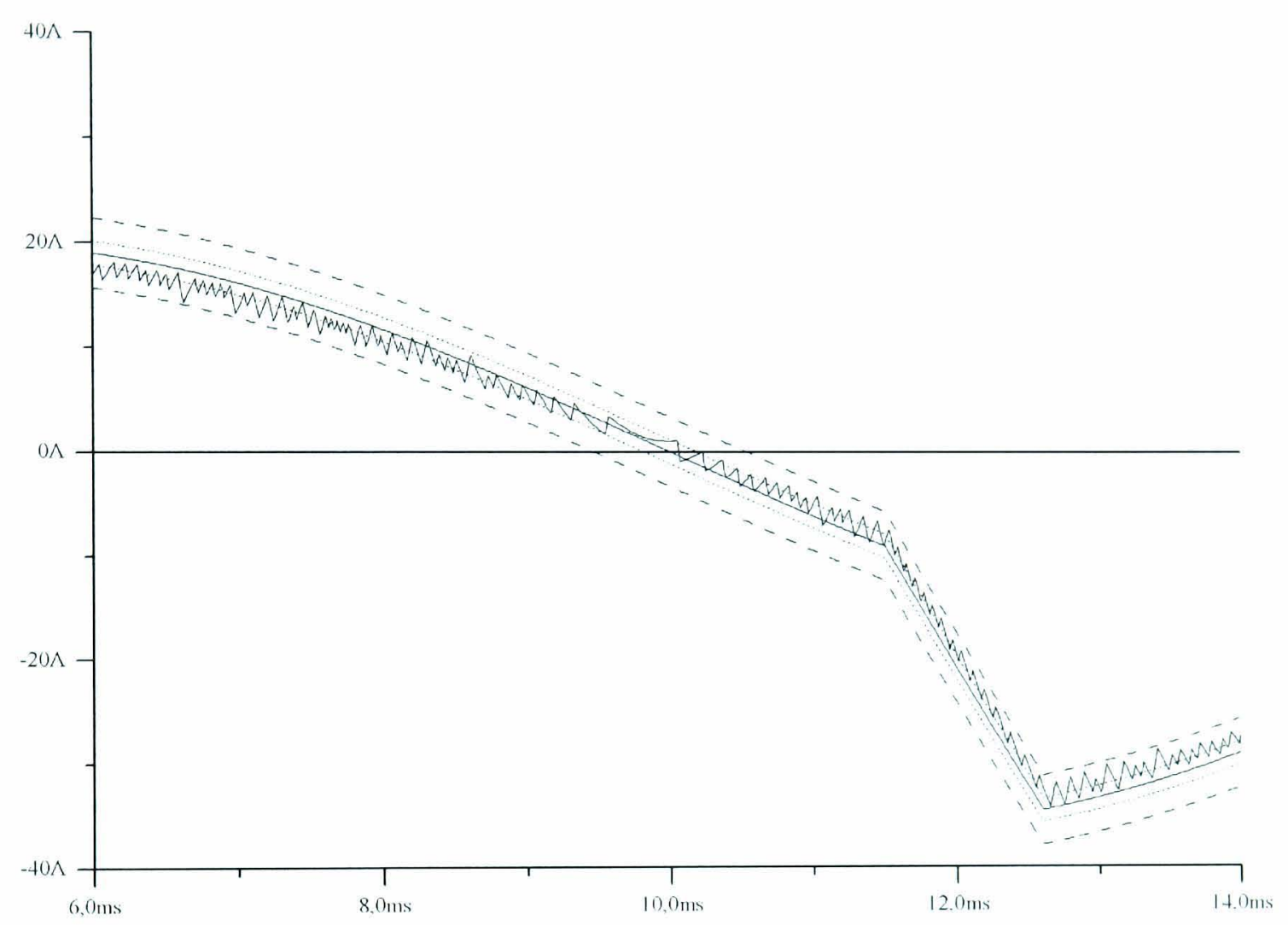

Fig.5.7 : Simulation results of the PWM hysterisis controller operation



Fig.5.8 : Load harmonic content after compensation with PWM techniques 
The simulated switching pattern of the switches in the case of the PWM-hysterisis is shown in Fig.5.9 with the pattern for each one of the switches. While the overall switching pattern for one complete cycle is given in Fig.5.10.

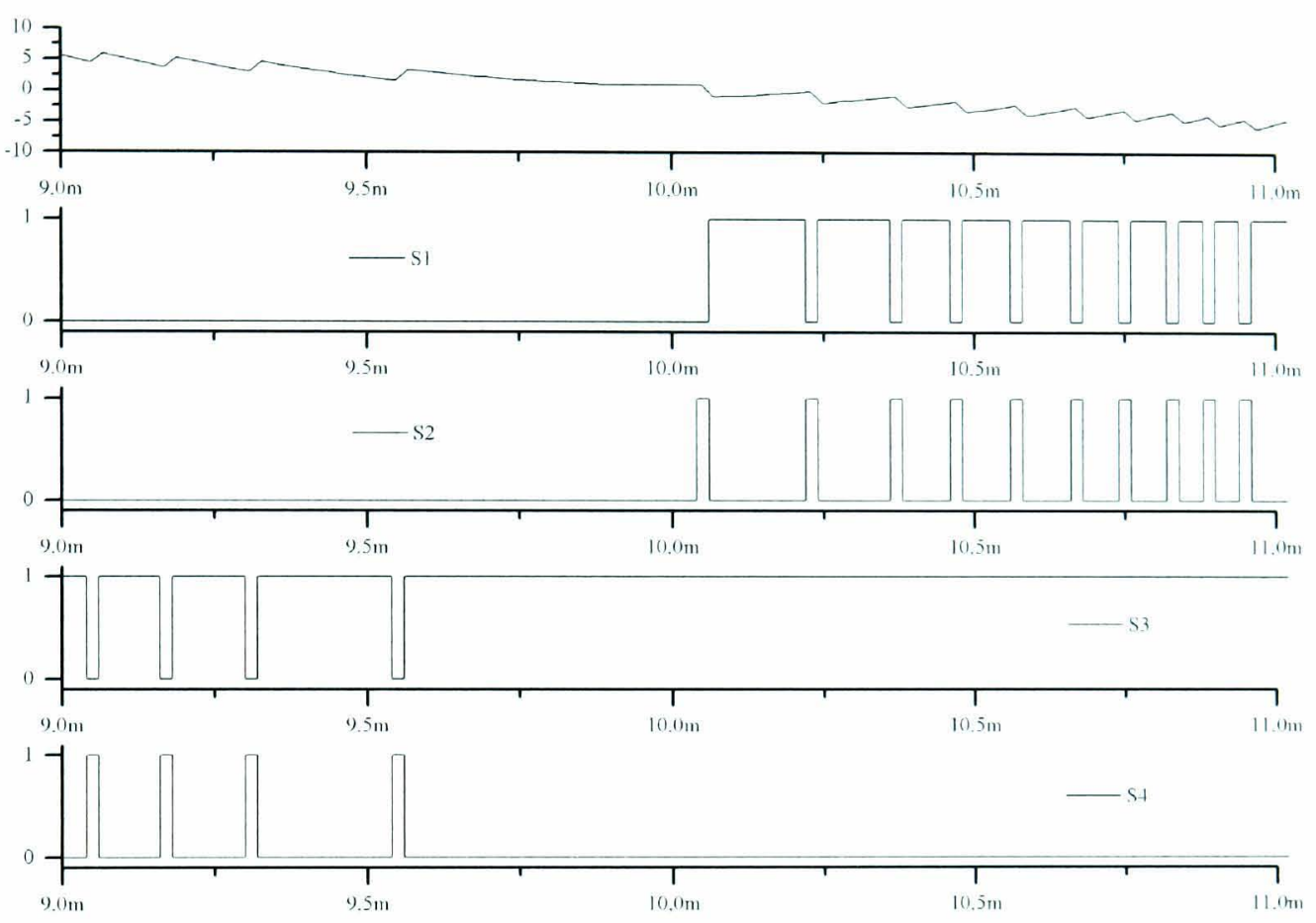

Fig. 5.9: Switching pattern of the switches and inverter current

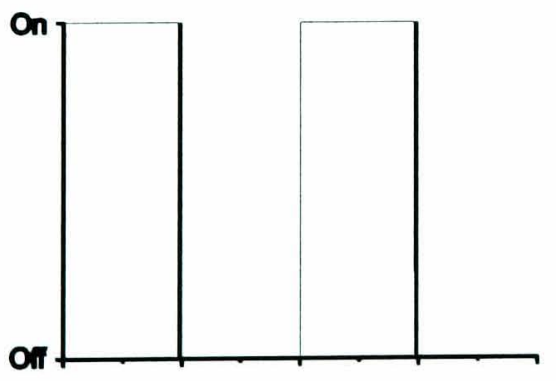

$S_{1}$

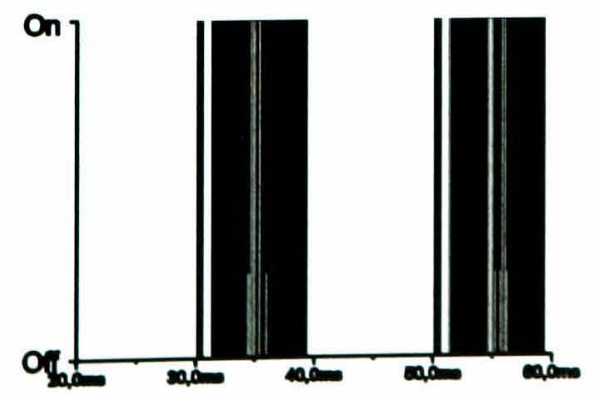

$S_{4}$

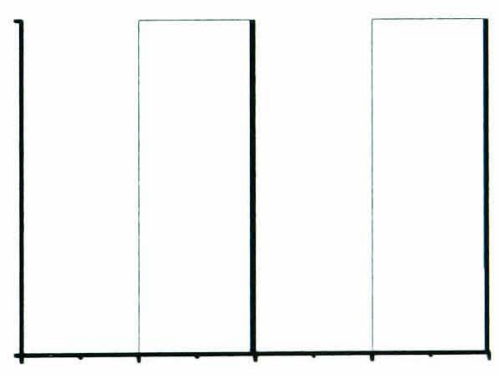

$S_{2}$

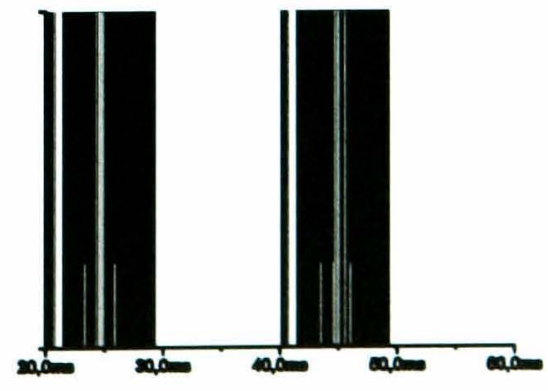

$S_{3}$

Fig. 5.10: Switching pattern of the IGBTs 
It is worthwhile to note that the resulting supply current after compensation for the case of using a hysterisis controller (Fig.5.6) is shown to be better from the point of view of low order harmonics levels, than the case of using the PWM-hysterisis controller (Fig.5.8), which does not eliminate all the low order harmonics.

Moreover, the current variations in Fig.5.7 for the PWM-hysterisis controller do not need to switch from positive to negative slopes (or vice versa) when it reaches the boundaries set by the hysterisis zones. Instead, the current switches between different slopes at the particular times when the integrated current (in Fig.5.4) reaches the limits. This case reduces the effects of the high frequency switching and reduces the amount of high order harmonics that is generated by the system.

This fact is obvious since the hysterisis controller reacts on the changes of the actual controlled value of the inverter current; while the PWM hysterisis is shown to react on the integral of the switching pattern, which renders its control more uniform however not extremely precise as the hysterisis controller case. These theoretical consideration are proved practically in the following chapter by the implementation of both controllers and comparing the current ripple resulting from each one of them.

However, in the next chapter it is shown that the incorporation of the electromagnetic interference criteria will prove that the use of the PWM-hysterisis controller is much better from the EMC point of view. This is the case for the high frequency analysis. which can not be accurately modelled on the computer when simulated at discrete time steps. 


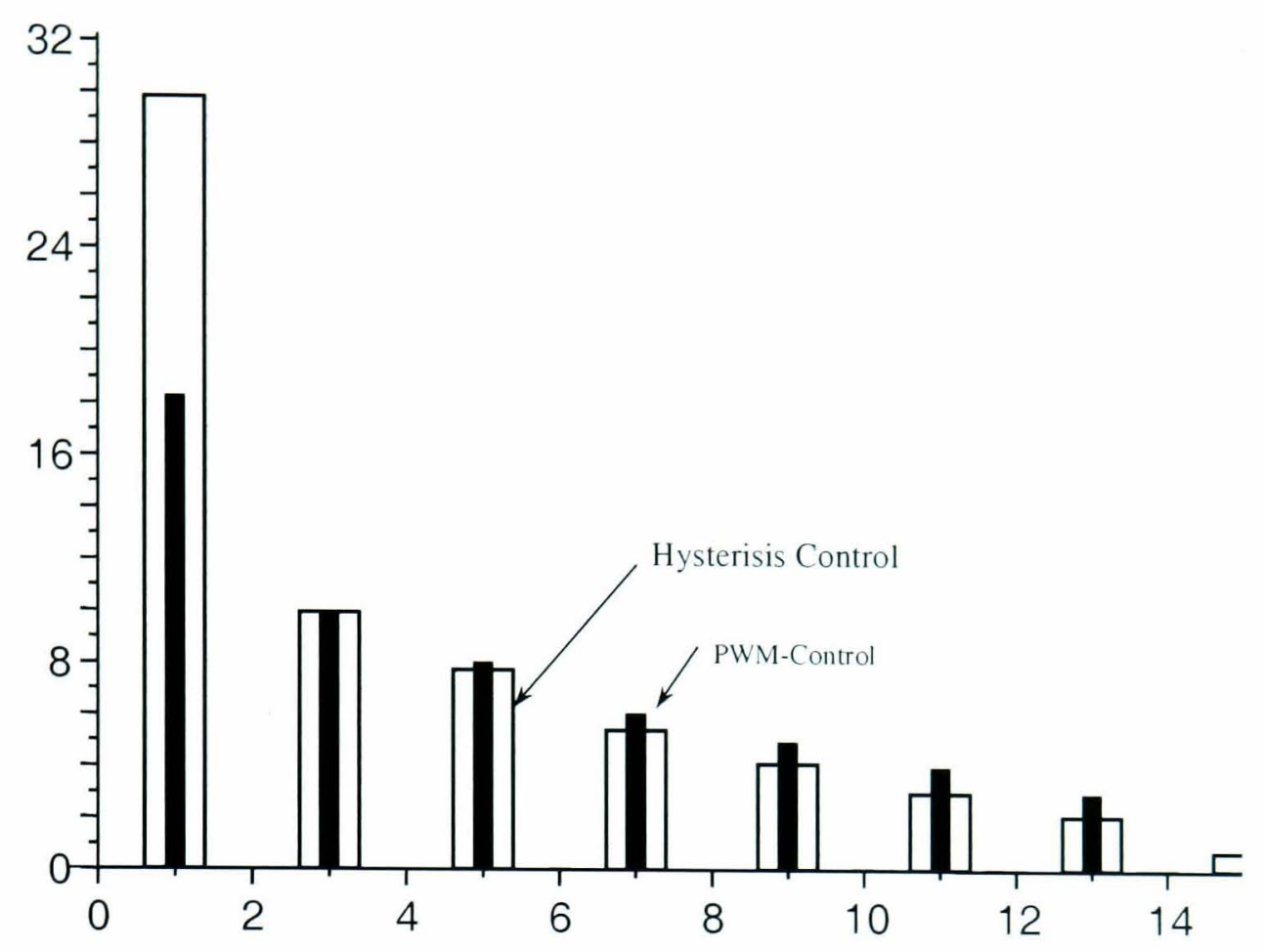

Fig.5.11: Prediction of the generated harmonics of both hysterisis and PWM techniques

Fig.5.11 shows the difference in compensation between the harmonics generated by the active filter for harmonic and reactive power compensation for both the cases of hysterisis controllers and PWM-hysterisis controllers for the low harmonic case (up to the $13^{\text {th }}$ harmonic).

\subsubsection{Effect of the passive filter}

In this section, the proposed circuit is simulated with and without the passive filter. Fig.5.12 shows the results of the circuit simulation without the passive filter. On the other hand, Fig.5.13 shows a better simulated-current-waveform without any superimposed high frequency switching harmonics for the case of the incorporation of the passive filter circuit in the simulation. It is obvious that the harmonic content of the supply voltage and supply current is increasingly reduced. 


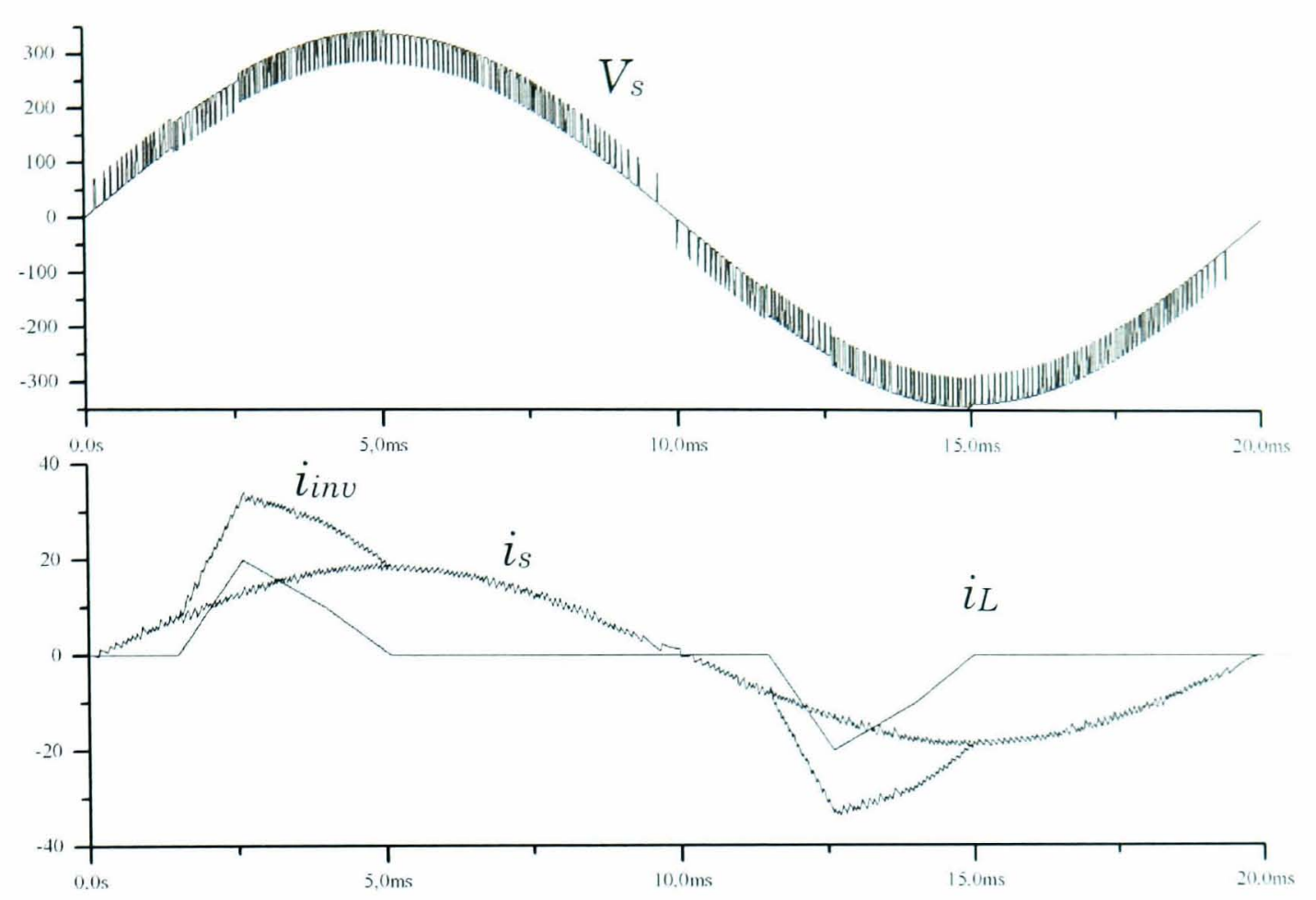

Fig.5.12 : Simulation results of the circuit without the passive filter

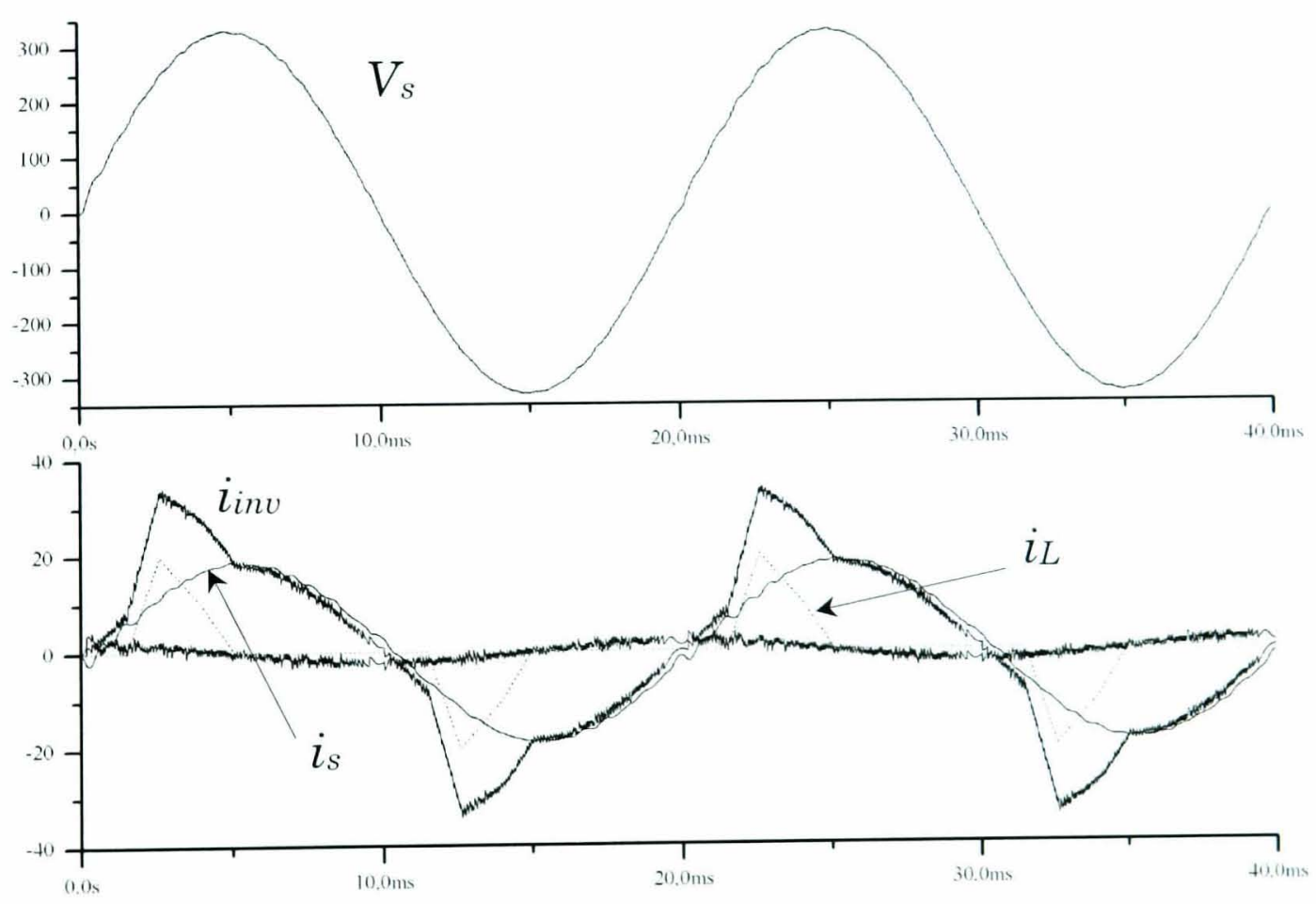

Fig.5.13: Simulation results of the circuit with the passive filter

\subsubsection{Harmonic measurements in real $10 \mathrm{kV}$ and $400 \mathrm{~V}$ power systems}

Total power consumption (active and reactive power) and harmonic measurements were carried out in a power distribution system. Long time periods were recorded. 
The transformer currents and all consumer currents were measured at the same time as with the voltage. The strongest harmonic voltage and current levels were at the $5^{\text {th }}$ and $7^{\text {th }}$ harmonics.

Using this data, the validity of the model along with the filter operation and performance were checked, as described below.

The measured values of the main network harmonic current were fed into the network model and simulated. Fig.5.14 and Fig.5.15 illustrate the significant reduction of the harmonics achieved before and after compensation. Fig.5.16 represents the instantaneous current and voltage waveforms, which were simulated for the filter operation.

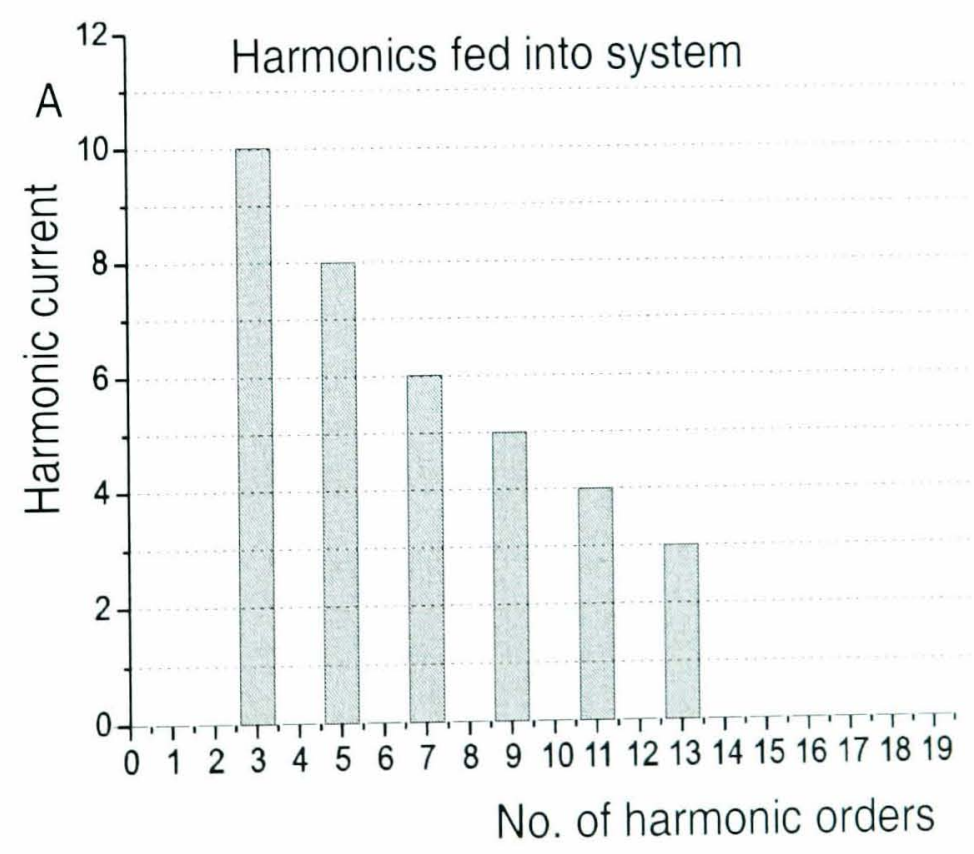

Fig.5.14: Harmonic current spectrum before compensation 


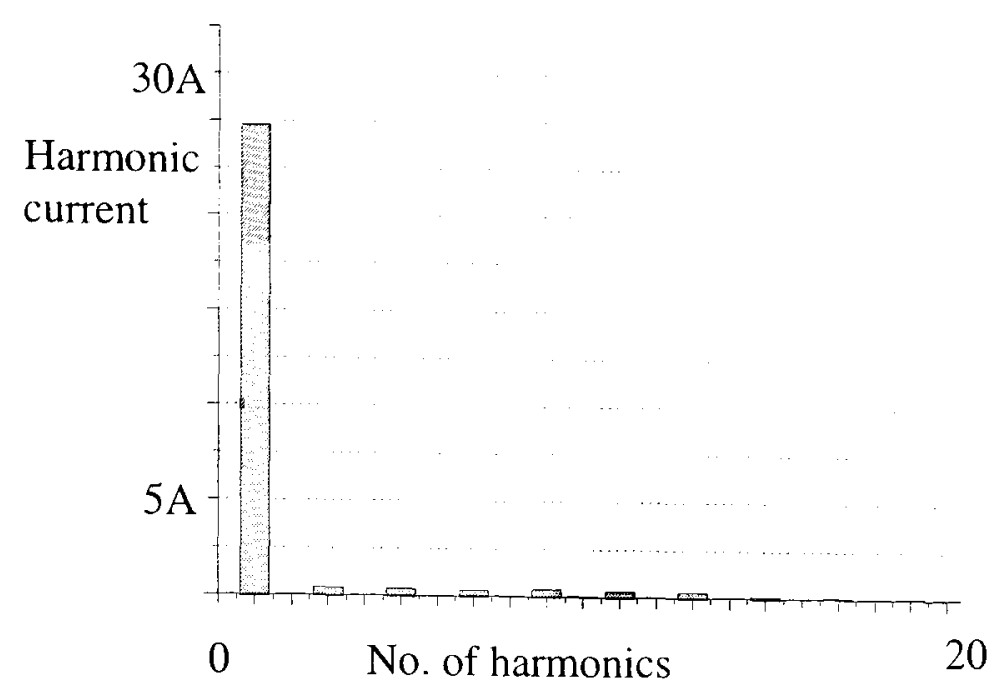

Fig. 5.15: Harmonic current spectrum after compensation

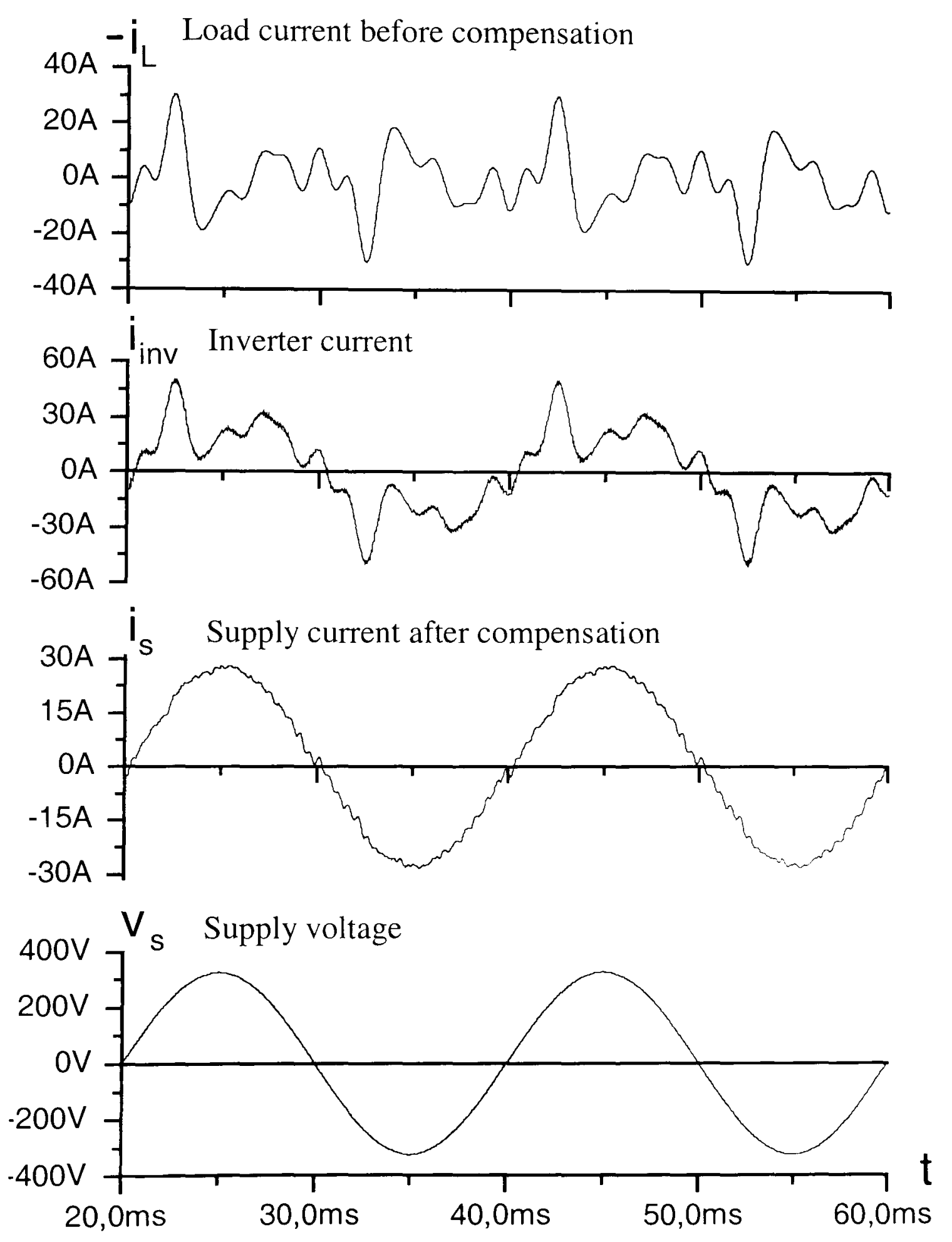

Fig.5.16: Performance of the system 


\subsubsection{Simulation results for an inductive-load in the power system}

Fig. 5.17 shows the simulation results for a given inductor load voltage and current spectrum before and after compensation and Fig. 5.18 and Fig. 5.19 harmonic spectrum before and after compensation respectively.

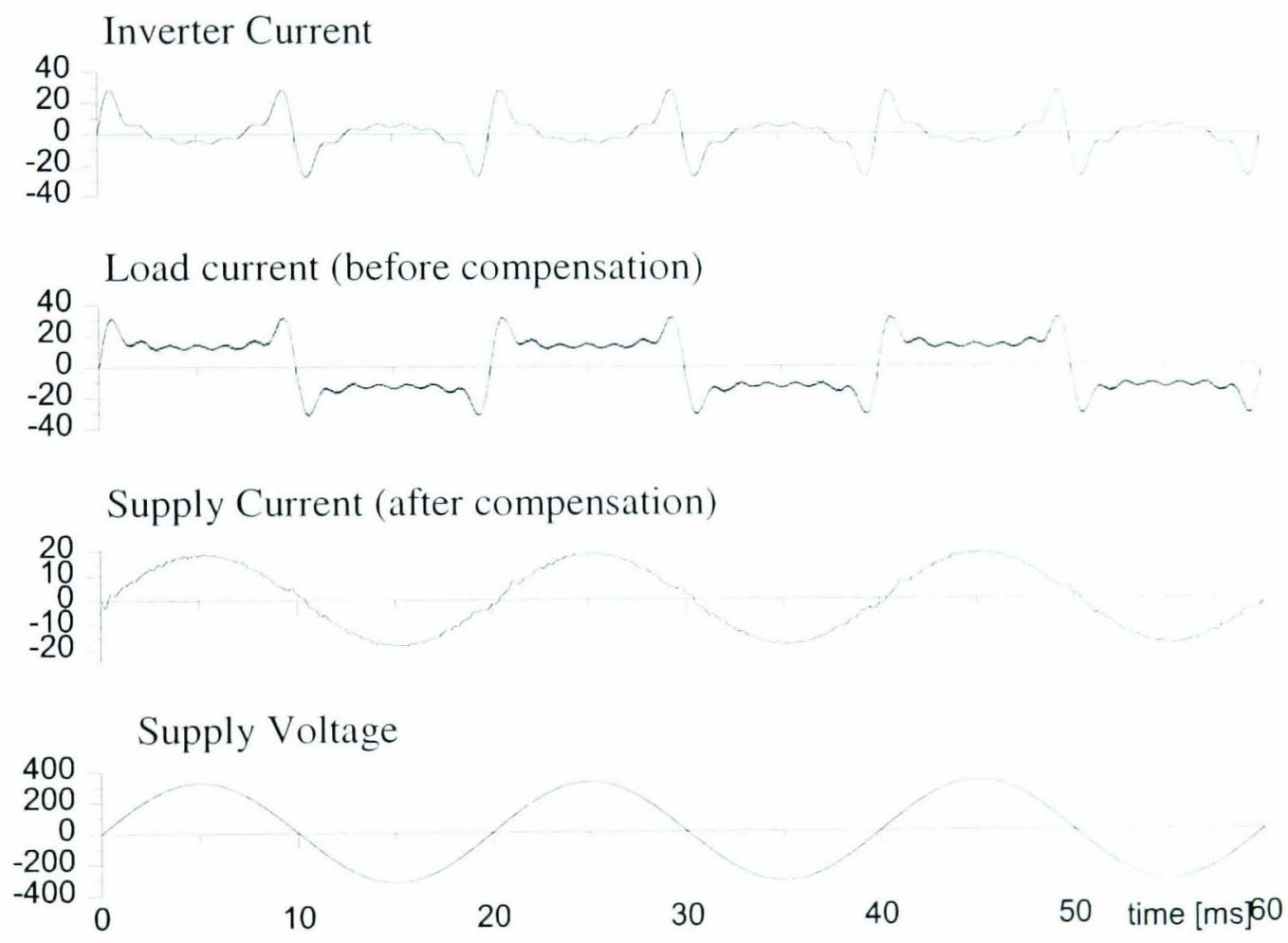

Fig. 5.17: Voltage and current spectrum before and after compensation

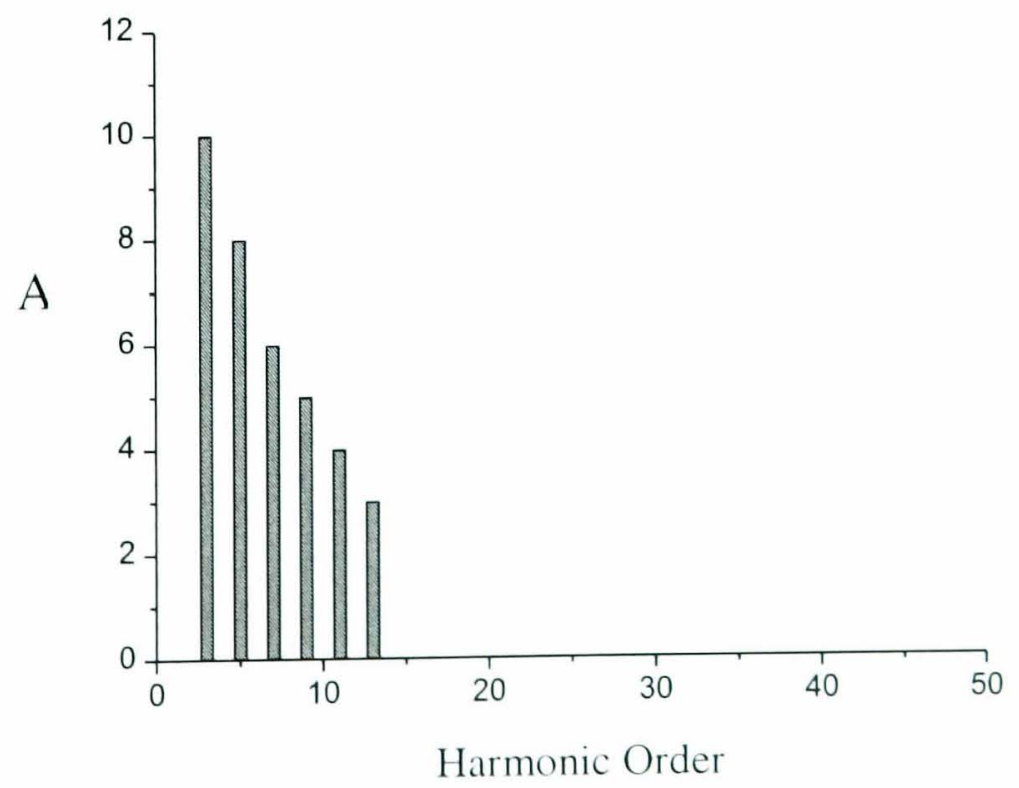

Fig. 5.18: Harmonic spectrum of Load Current before Compensation 


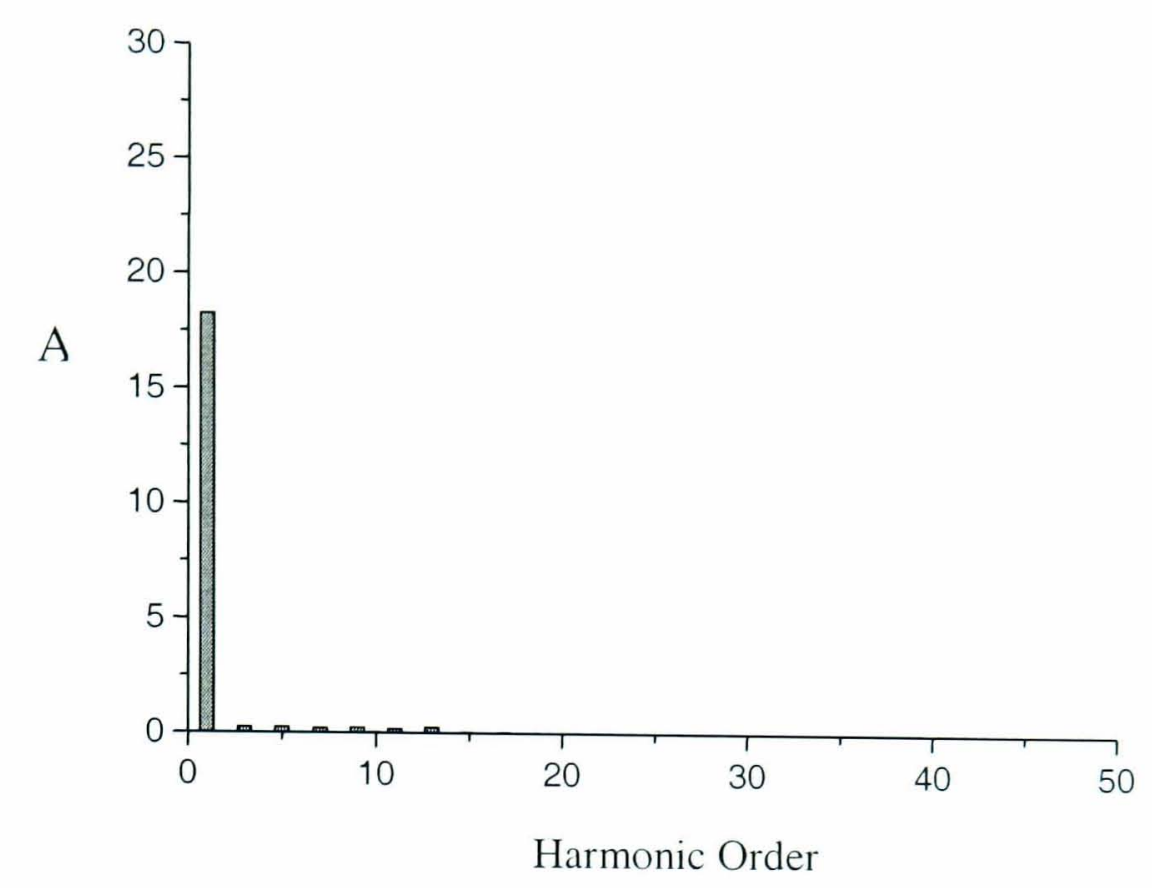

Fig. 5.19: Harmonic spectrum after compensation

\subsubsection{Simulation Results for Given Non-Linear Load Output}

The nonlinear load shown in Fig. 5.20 is representative of most electronic equipment in the industry and computing systems. The proposed system filters the harmonics successfully. Figures 5.21 and 5.22 show harmonic current spectrum before and after compensation.
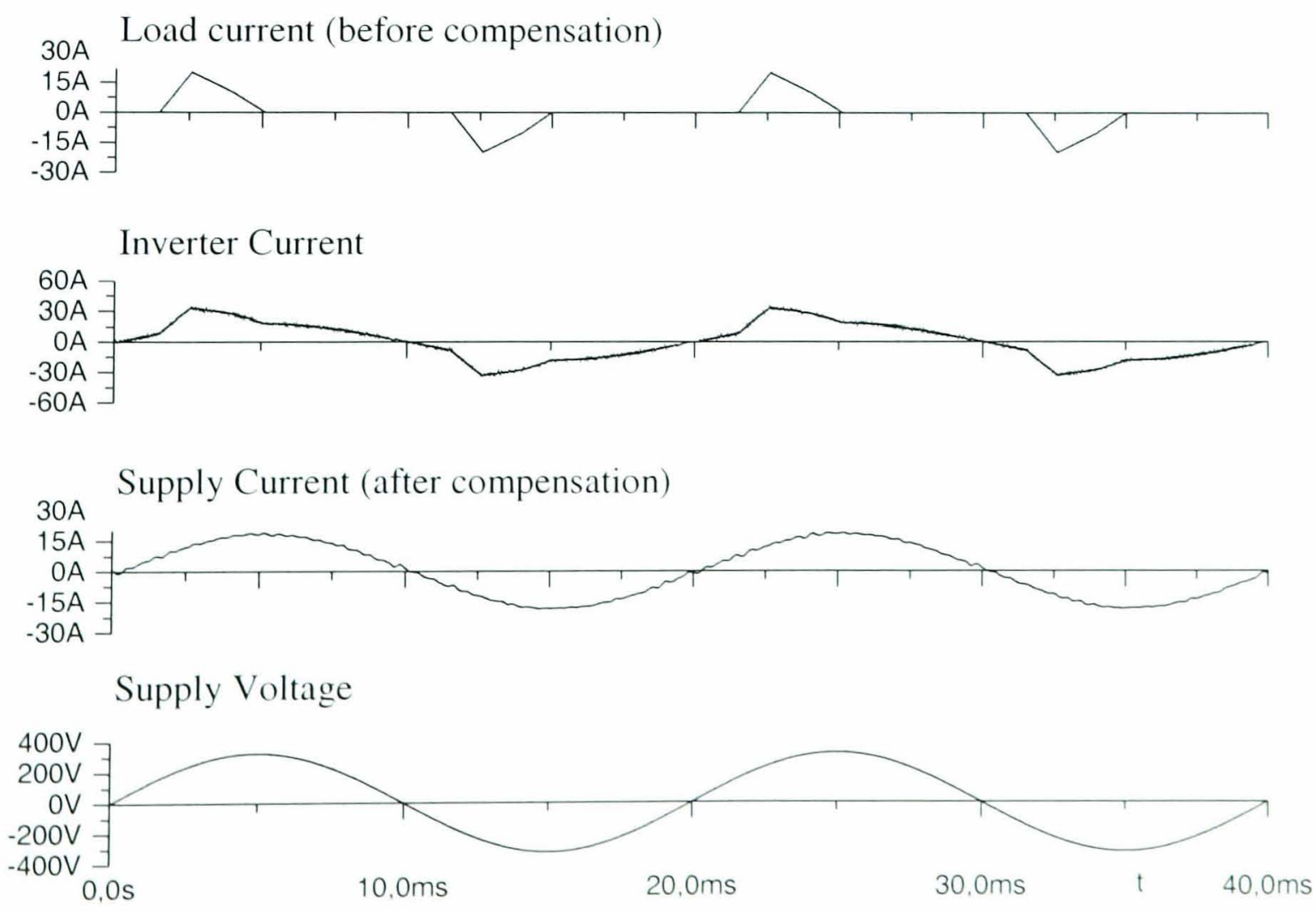

Fig.5.20: Voltage and current spectrum before and after compensation 


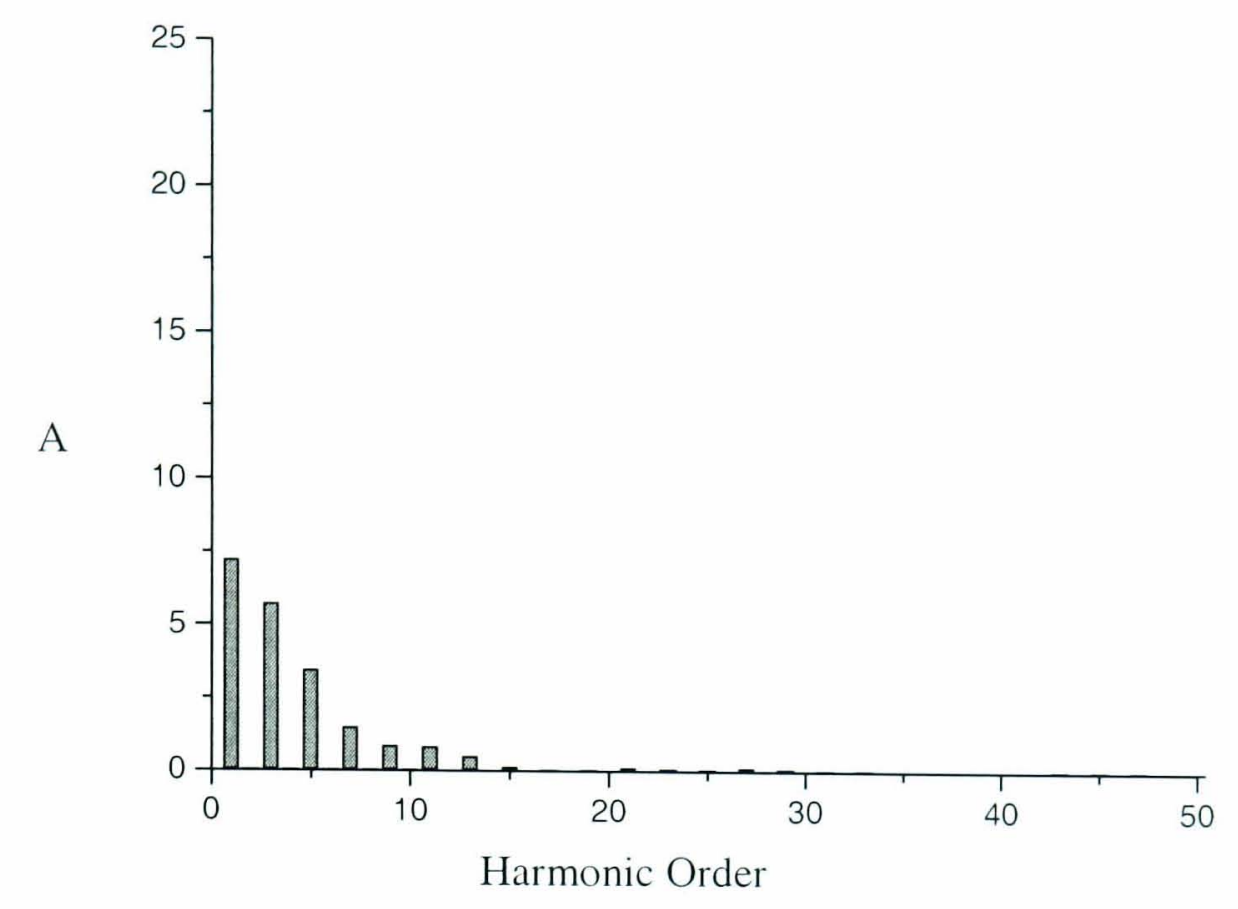

Fig.5.21: Harmonic current spectrum before compensation

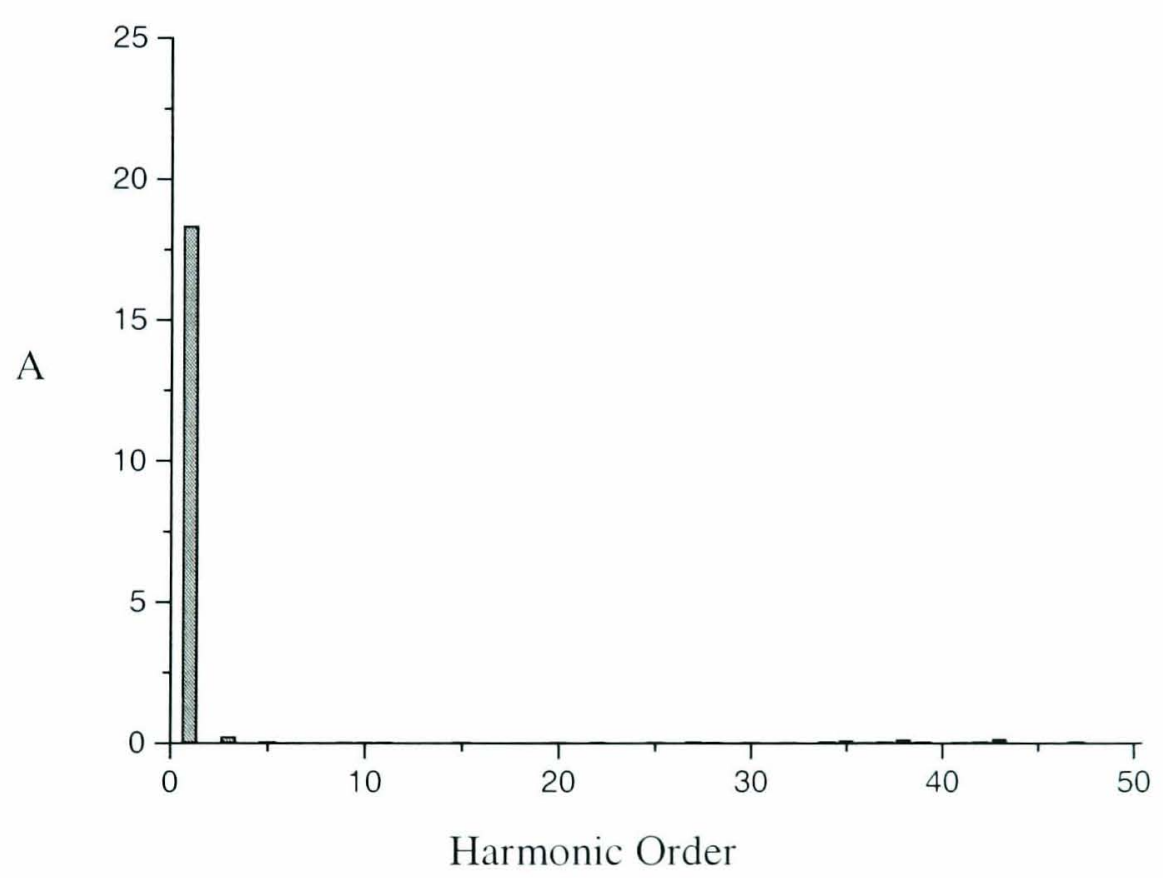

Fig.5.22: Harmonic Current Spectrum after Compensation

\subsection{Summary}

This chapter described the process of simulating the proposed system. Various nonlinear loads were tested using the dedicated simulation software and the system is shown to operate properly according to the design. The implementation of this power system conditioner will follow in the next chapter. 


\section{Chapter 6}

\section{Experimental Set-up and Practical Results}

\subsection{Introduction}

In the previous chapter, the simulation of the proposed system was performed and this proved to be successful in eliminating the need for two devices: reactive power compensator and harmonic eliminator. The simulation showed that the device does not generate any low-frequency harmonics while eliminating the reactive power. This chapter deals with the practical implementation of the proposed system. It describes the practical prototype implementation and the control process according to the simulated data of the previous chapter.

A single-phase active filter was built in an experimental laboratory to prove the simulation results and to verify the control system and network topology. The results, which will be presented later on in this chapter, will show the voltage and current before and after compensation as well as the harmonic current spectrum before and after compensation.

\subsection{Choice of Switching Devices}

Gate/base controlled components such as bipolar transistors, power MOSFETs. GTOs and IGBTs are used for controlling currents, voltages and switching frequencies [159]. GTOs 
can only achieve their maximum output up to $2 \mathrm{kHz}$. The bipolar transistor has lower onstate power losses when switched on, particularly for high base impedance types.

Power MOSFETs are quick and can be switched on and off at relatively very high switching frequencies, but their on-state power losses are distinctly higher than those of other types. The control of MOSFETs is much easier than that of bipolar transistors. The advantages of the bipolar transistor and the MOSFET are combined in the Insulated Gate Bipolar Transistor (IGBT). The difference is the additional, strongly doped layer on the collector side.

When examining control methods for power converters, the permissible switching frequencies of semi-conductors is of great importance. It substantially affects the approximation of the converter voltage and current curves to the ideal design values.

\subsection{Inverter and AC-Filter Design}

In this section, the required elements of the single-phase configuration of the proposed filter are calculated and designed. In order to design an AC-filter, IEC standards must be followed. The inverter output current has to keep line perturbation below the permitted levels. Frequencies below $2 \mathrm{kHz}$ and above $10 \mathrm{kHz}$ have to be taken into account. The precise working conditions of the inverter and passive filter depend on the proper choice of the circuit characteristics such as IGBTs, drivers, resistors, capacitors and other ICcircuits. Often some of them are limiting factors. For this reason, a construction guide for 
the inverter and AC-filter design practical procedures is given in the flow charts of Fig.6.1 and Fig.6.2, respectively.

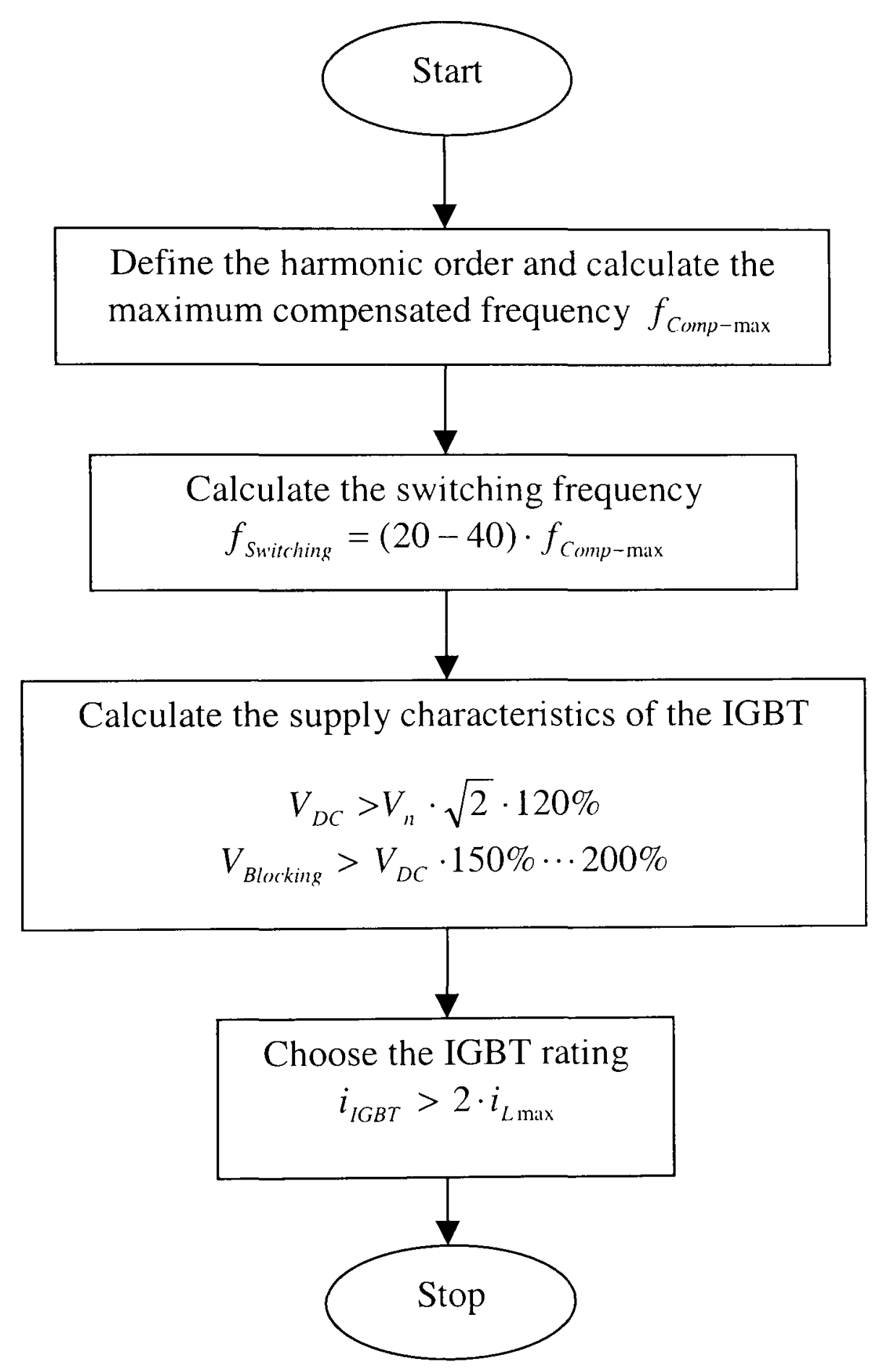

Fig.6.1: Inverter-Design Procedure 




Fig.6.2: AC-Filter Design Procedure

\subsection{Experimental Set-up}

The complete block diagram of the proposed system was presented in chapter 3. Fig.3.1 represents the block diagram of the control system. The block diagram shows ten main 
sections constituting the active/reactive compensator. The practical implementation of the system must include all these blocks in order to realise the function of the circuit.

The harmonic current generated by the non-linear load is detected and fed-back to the reference current estimator, in conjunction with the other system variables. The resulting reference signal drives the overall system controller, which, in turn, generates the control output necessary for the switching pattern. The resulting filter current is also detected and fed back to the controller. The power circuit of the inverter and the passive filter circuits are then connected to the power system at the PCC. The power circuit of the proposed system is presented in Fig.6.3.

All measurements were carried out using the set-up shown in Fig.6.3 consisting of a nonlinear load, an AC supply, complete control system, IGBTs for a single-phase inverter circuit as well as connection points for measurements.

The custom-designed and built control circuits contain commercially available electronic components, which are connected together to generate reference and feedback currents with a hysterisis-band controlled PWM signal driven at a fixed switching frequency $(20 \mathrm{kHz})$. Pulse width modulation is used to eliminate the effects of electromagnetic interference. The hysterisis current control method has an excellent dynamic response as it is equipped with a very fast control mechanism. The printed circuit board is shown in Appendix D-1, which contains the terminals for the required inputs, outputs and signals. The clectronic circuits operate at \pm 15 Volts. The IGBT driver circuit pin description is 
Non-linear load

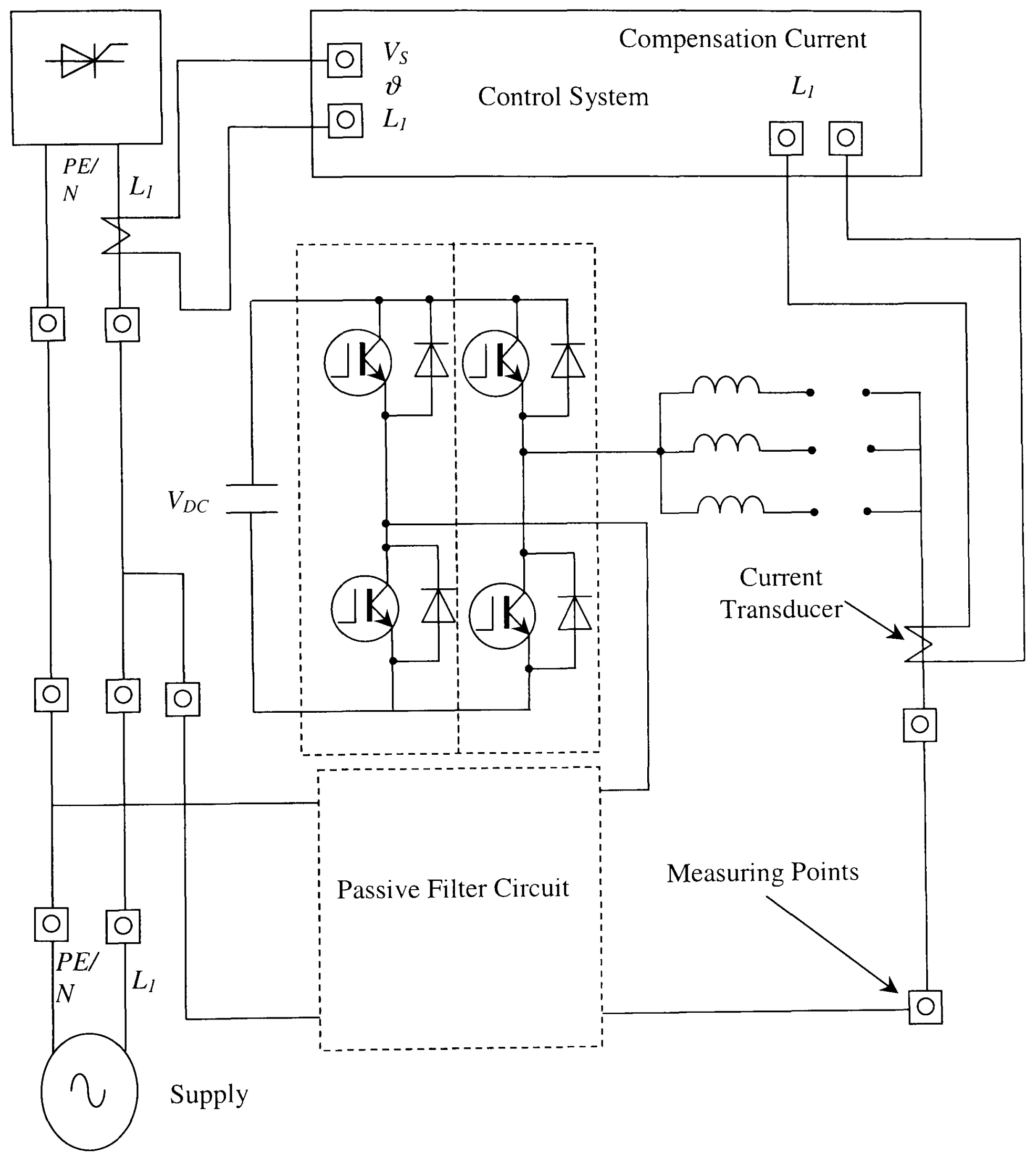

Fig. 6.3: Practical Layout for the Experimental Set-up

presented in Appendix D-2 shows the main network pin description and the driver board.

The currents and voltages are detected using current and voltage transducers with the appropriate ratings chosen according to the load rating and then connected to the control 
unit (Appendix D-3). According to power supply companies power factor of the plant must be at least 0.95 . For this reason a compensation adjustment ( $\cos \varphi$-adjustment) is designed (Table D.5 to Table D.7).

Moreover, the power circuit is protected from short circuits and faults with the aid of fuses and relays. A protection system is also included against transients and lightning surges, which may totally damage electronic equipment. Appendix $\mathrm{E}$ shows the power and control circuit. Temperature sensors are installed in the "hot spots" to detect interior temperatures of the inverter (set at $70^{\circ} \mathrm{C}$ ) and passive filter circuit. Under any fault, the protection system will disconnect the power and isolate the whole circuit. An Overview of the complete system is given in the Appendix F.

It is worthwhile to note that the state of the inverter capacitor has to be considered at the start of the operation. This is due to the fact that the operation of this capacitor can be divided into three modes (Fig.6.4). Mode 1 is the "switch-on" condition where the DC capacitor charge is at its minimum. In this case, energy flows from the ac mains into the DC capacitor system (charging operation). This mode is normally the case when the inverter is switched on for the first time. Charges start building up across the capacitor until its voltage is equal to the nominal value $(800 \mathrm{~V})$. At this point the operation of the circuit can resume. Mode 2 relates to "compensation", where energy flows from the DC capacitor into the mains (discharge operation). The third and last mode (Mode 3) is the "switch-off" condition where the energy across the DC capacitor must be dissipated in an 
external resistor. The flow chart in Fig.6.4 explains the operation of each mode and the transfer from one mode to the other with the conditions needed for each mode to occur.

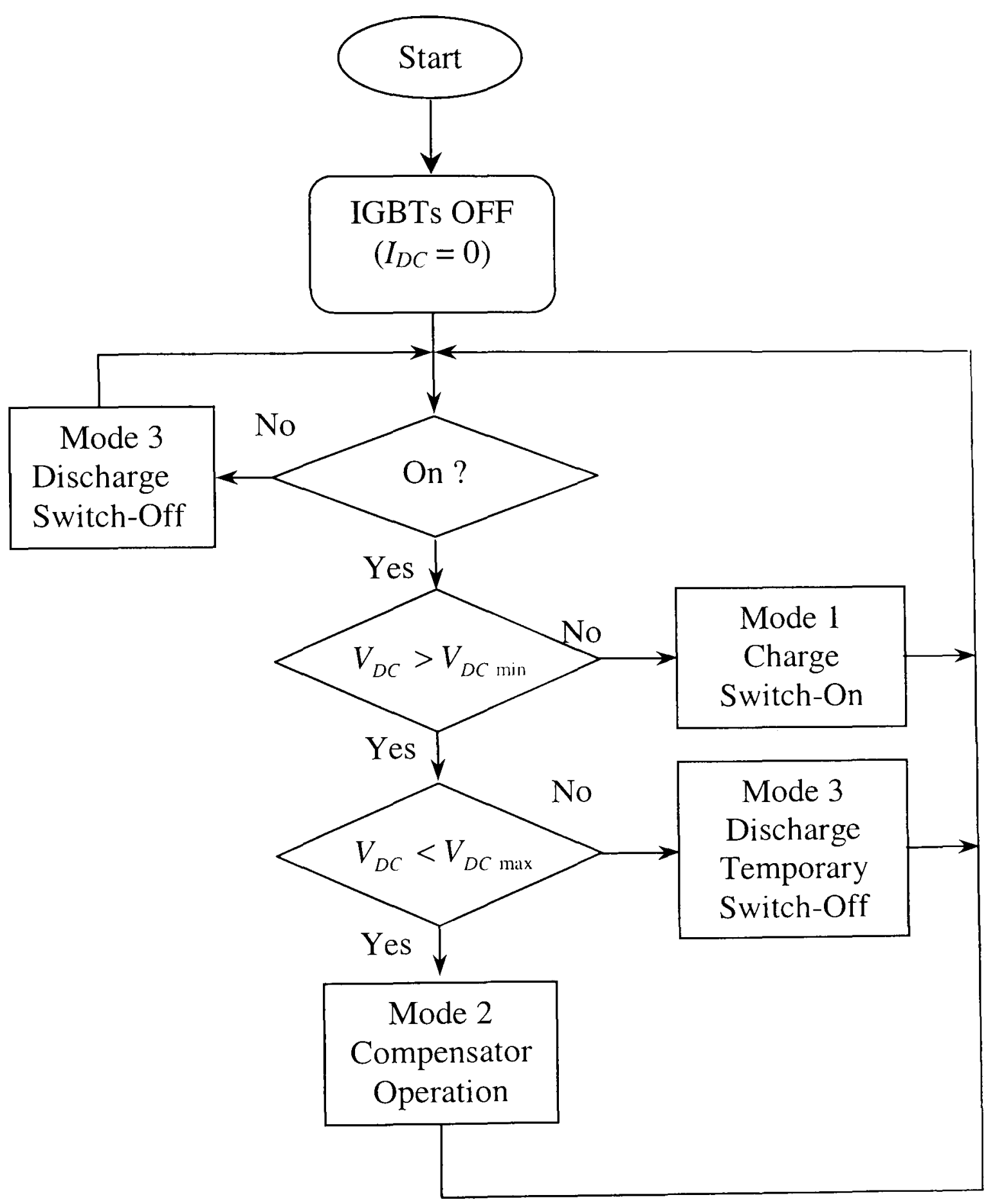

Fig.6.4: Flow Chart for the DC Capacitor Operation

In power system applications, high efficiency of the inverter circuit is desirable. In the following, the efficiency is determined by the device and filter losses. For rising switching frequency, device losses increase and filter effort decreases: thus the filter 
losses decrease. To find an optimum switching frequency, inverter inductance value and filter design, the losses must be calculated and measured.

\subsection{Practical Results}

The practical implementation of the above system generates a large amount of practical results, which are subdivided in the following sections.

\subsubsection{EMC Compatibility and Choice of PWM and Hysterisis Control}

Besides non-linear loads, inverters cause high frequency harmonics, noise propagation and malfunction in the power system. Electronics systems in the surroundings of the inverter react to the presence of high power harmonics in the range of up to $10 \mathrm{kHz}$ and low power harmonics between $10 \mathrm{kHz}$ and $30 \mathrm{kHz}$. These are disturbance voltages due to switching high amounts of currents and voltages on and off and are given in $d B / \mu V$. In order to reduce these effects, the special passive filter circuit and the PWM-hysterisis current control techniques were used.

It is recommended by the standards to comply with the harmonic and EMI regulations. In order to ensure that the proposed system meets the regulations, the high frequency components of the inverter and supply currents were measured using a spectrum analyser.

The EMI generated by the proposed system was measured when employing both hysterisis and PWM techniques. Fig.6.5 shows the results of the electromagnetic 
emission without the passive filter. The emitted interference is decreased about $30 \mathrm{~dB} / \mathrm{V}$ as shown in Fig.6.6, when using the PWM-hysterisis. This can be interpreted to the fact that the insertion of the zero switching states during the delays explained in chapter 3 causes the voltage signal to switch from $\pm V_{d c}$ to 0 and not to the other polarity which at least halves the amount of EMI generated. The insertion of the switching delay within the switching subcycles ensures another reduction. The passive filter designed traps the high frequency harmonics as shown in the Bode plots of chapter 3. It stops these harmonics from penetrating into the supply and hence further reduces the EMI generated by the system.

Permissible level of electromagnetic emissions of the switching power devices is given in Fig.6.7, in conjunction with the emitted harmonics. These indicate that the passive filter and the proposed switching technique meet the EMI regulations.

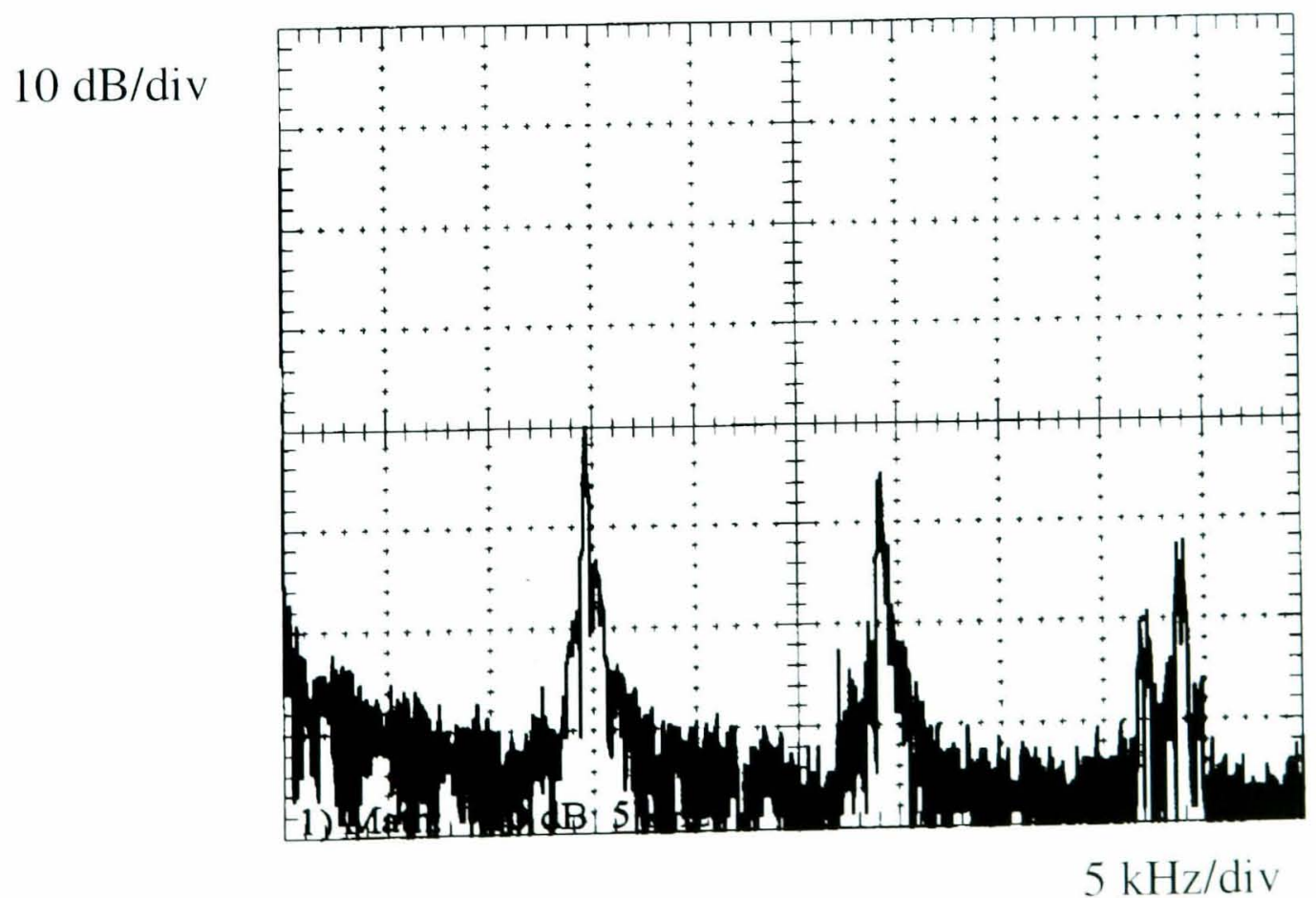

Fig.6.5: Practical results of the electromagnetic emission without the passive filter 


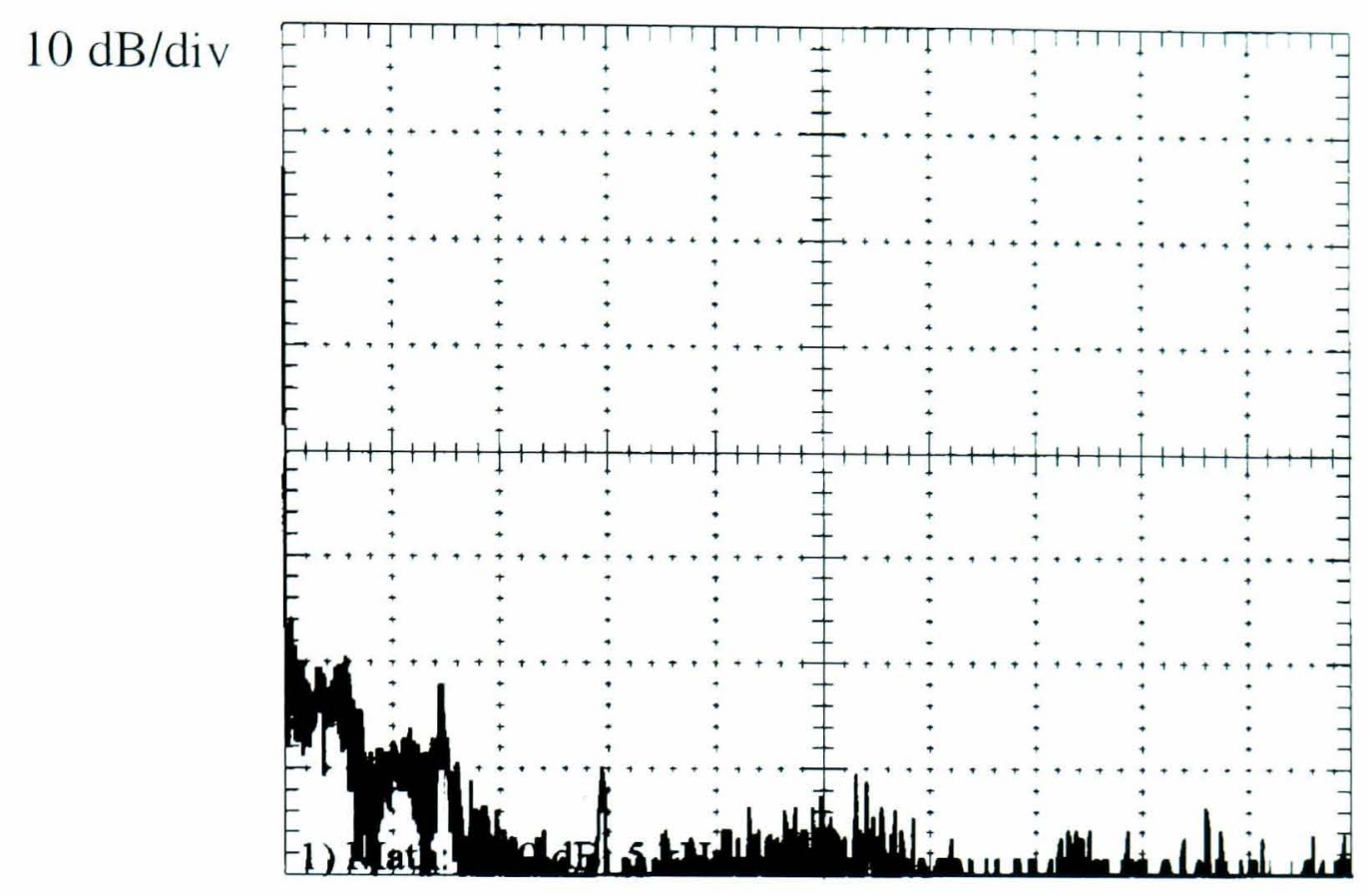

$5 \mathrm{kHz} / \mathrm{div}$

Fig.6.6: Practical results of the electromagnetic emission with the passive filter

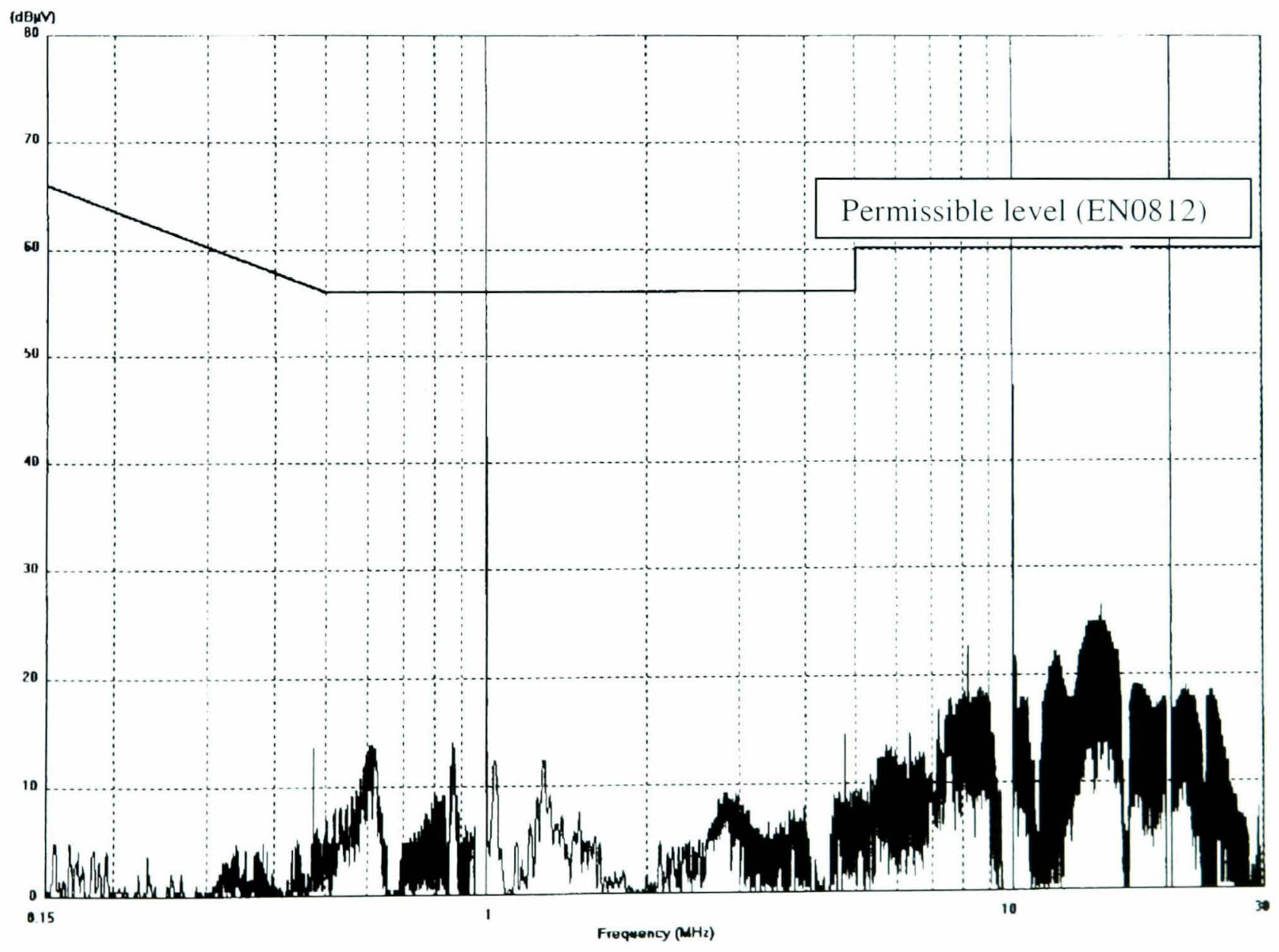

Fig.6.7: Permissible level of the electromagnetic emissions of the switching power devices 


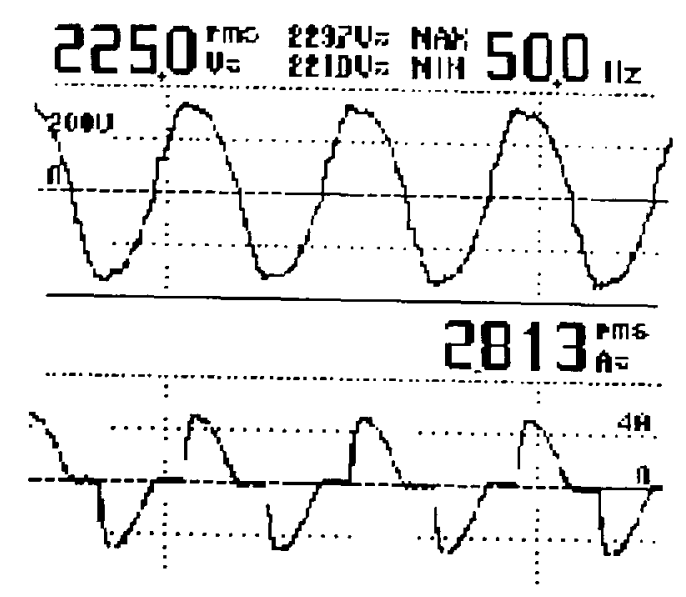

(a)

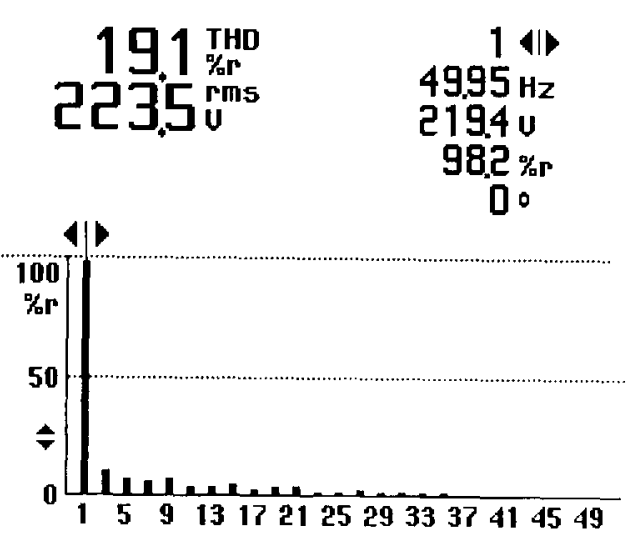

(b)

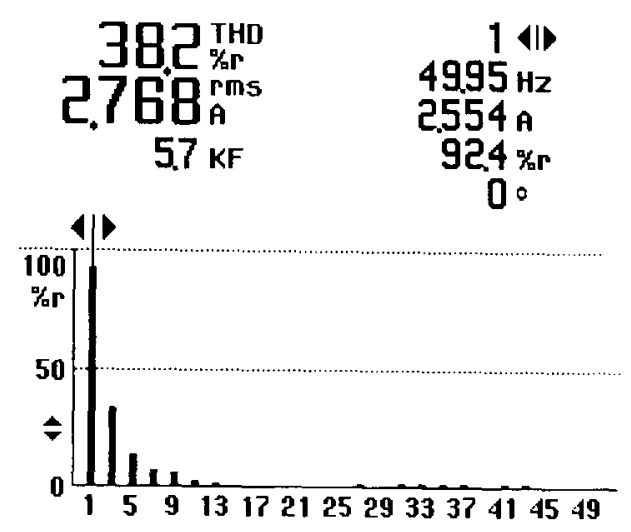

(c)

Fig.6.8: Load Current waveform and its harmonic content

\subsubsection{Choice of Inverter Inductance}

Fig.6.8-a shows a non-linear load current characteristics with the supply voltage, which was used for all measurements. This is a typical thyristor bridge with an inductive load on the DC side. The triggering angle for the thyristor bridge is $60^{\circ}$. This is a typical nonlinear load with difficult compensating characteristics that contains both reactive power compensation as well as harmonic compensation. It is considered in these cases by the practical implementation for all the test conditions. Fig.6.8-b shows the harmonic 
distortion in the supply voltage. Fig.6.8-c shows the harmonic content of the load current, which represents the non-linear components that will be removed by employing the proposed circuit. Note the presence of more than $30 \% 3^{\text {rd }}$ harmonic and $20 \% 5^{\text {th }}$ harmonics, leading to a $38.2 \%$ total harmonic distortion (THD).

\subsubsection{Nonlinear load with $L_{i m \cdot}=4 \mathrm{mH}$}

In Fig.6.9, an inverter inductance of $4 \mathrm{mH}$ was used for the measurement of this case. This chosen value is conformal to that of calculations. The curves of Fig.6.9-b show that the compensated current is not sinusoidal. However, there exists a large value notch in the middle of each half cycle, which is superimposed on the sinusoidal current waveform. Fig.6.9-c shows the harmonic spectrum of the compensated current and it still contains a considerable amount of $3^{\text {rd }}, 5^{\text {th }}$ and $7^{\text {th }}$ harmonics. The choice of $4 \mathrm{mH}$ inductance was mainly due to the simulation, which normally neglects some of the nonlinear effects of the system. The following two sections use reduced values of inductance in order to improve the system performance and the harmonic elimination.

\subsubsection{Nonlinear load with $L_{i m}=2 \mathrm{mH}$}

Fig.6.10 shows the current waveforms as well as the harmonics contents for the same case as above with a smaller value of inverter inductance $\left(L_{i n v}=2 m H\right)$. It is very clear that a smaller value of inductance has helped a great in reducing the nonlinearity in the supply current. 
It is also worthwhile to note that the hysterisis band increases with the decreasing inverter inductance. This is due to the fact that the rate of current rise is increased by reducing inverter inductance. In order to keep the same switching frequency of the system, the allowable hysterisis error band must be increased. This of course does not affect the low order harmonic content of the system as the switching frequency is fixed in the range of $16-20 \mathrm{kHz}$, which is far beyond the low frequency range.

\subsubsection{Non-linear Load with $L_{i n v}=1 \mathrm{mH}$}

This is the ultimate operating condition of the load. It can be seen that the proposed system operates satisfactorily from the point of view of generating a relatively smooth sinusoidal waveform (neglecting the superimposed switching frequency) (Fig. 6.11). The generated waveform can accomplish the required task of eliminating power factor as well as harmonics from the power system, as will be discussed further in the next subsection. It is shown, in this case, that the tuning of the optimal values of inductance calculated theoretically can be performed on the prototype. This is quite advantageous for the final fine tuning, since the simulation does not take the nonlinear effects of the inverter such as the saturation of the output voltage and the switching time delays of the switches. In addition the losses of the system are calculated as mentioned above for certain operating conditions, which do not include the snubber circuits across the IGBTs. All this implies that the theoretical computation has to be fine-tuned on the implemented prototype as shown above. In the next part of the load test, the value of $1 \mathrm{mH}$ will be adopted since it generated the best results for these operating conditions. 


\subsubsection{Power Factor Correction Case $\left(L_{i n v}=1 \mathrm{mH}\right)$}

Fig.6.12 shows the measured results for power factor correction at 0.86 and at 1.00 as well as the harmonic spectrum of the supply current after compensation. This case presents the ultimate compensation performed by the system. The power factor correction, presented in the first case of Fig.6.12-a, shows the effect of the partial compensation from the load current shown above to the case of 0.86 power factor. The overall unity power factor correction is shown in Fig.6.12-b to have compensated for the harmonics as well as the reactive power. In this case the measuring device still reads the value of $45 \mathrm{VAR}$ as a reactive power while the power factor is shown to be unity. This is due to the method the measuring device uses to perform the sampling of the signal. The high frequency harmonics in the current are interpreted, by the measuring device, as being reactive power. On the other hand, the power factor shown as unity is the interpretation of the power factor of the fundamental component of the compensated current which in this case is in phase with the supply voltage and hence has generated the unity power factor as a result of the compensation. 


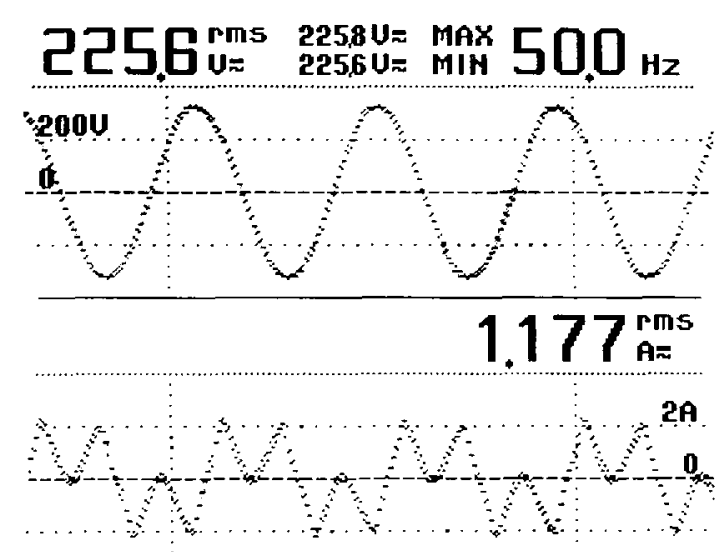

(a) Inverter Current

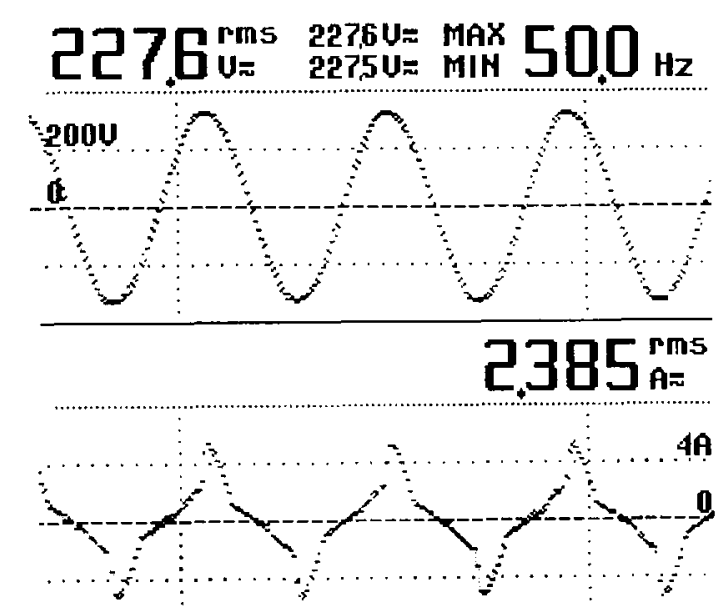

(b) Compensated Current

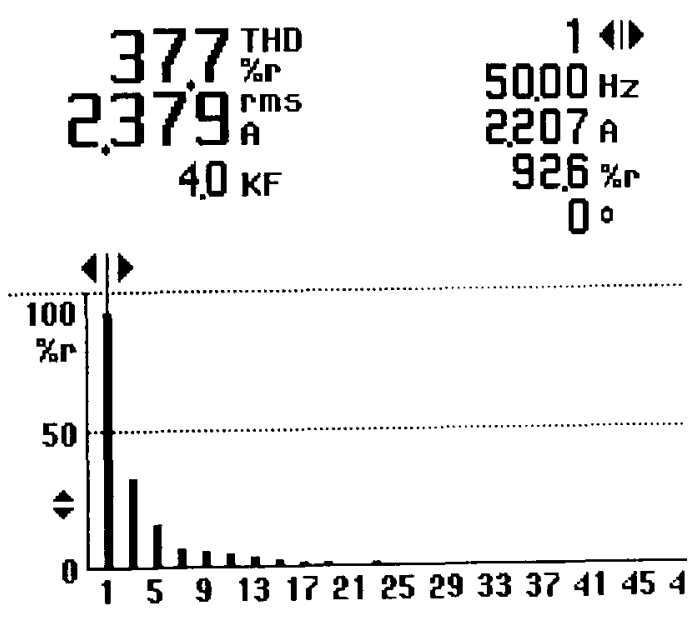

(c) Harmonic Spectrum

Fig.6.9: Loading condition for $\left(L_{m,}=+m H\right)$ 


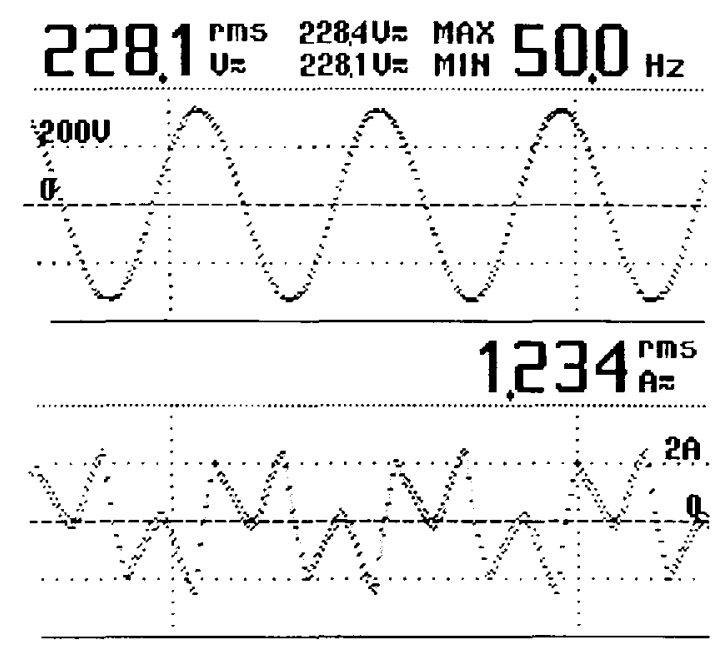

(a) Inverter Current

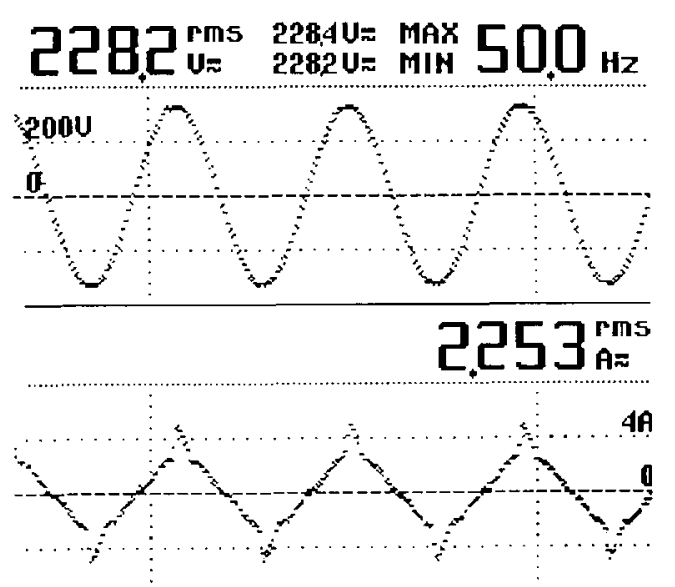

(b) Compensated Current

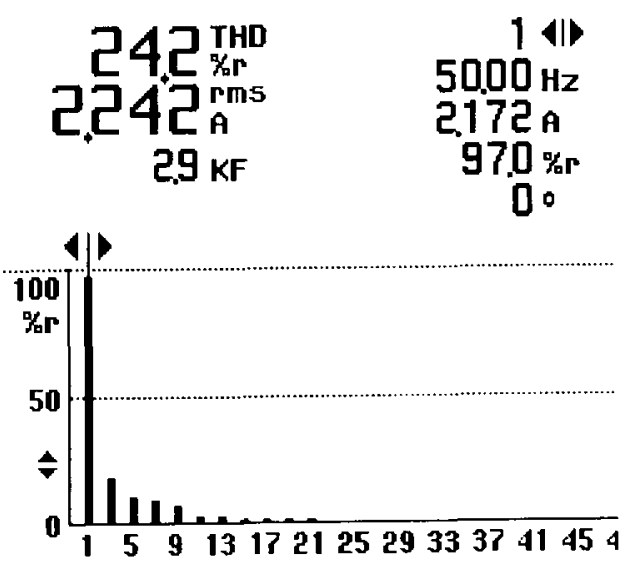

(c) Harmonic Spectrum

Fig.6.10: Loading condition for $\left(L_{i m}=2 \mathrm{mH}\right)$ 


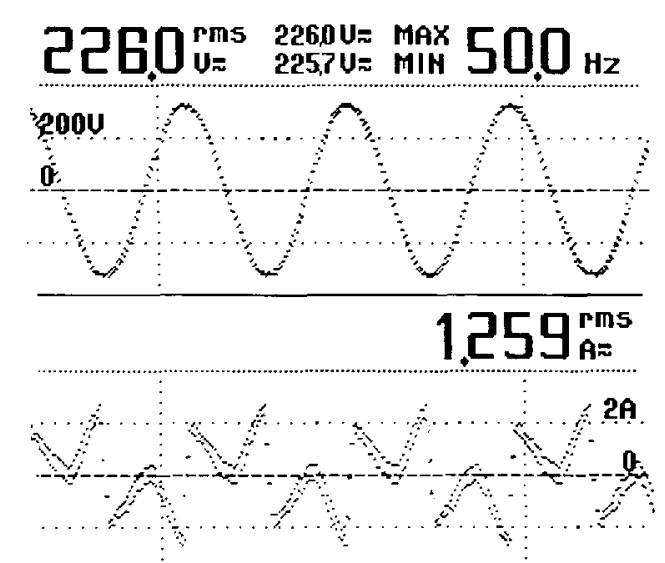

(a) Inverter Current

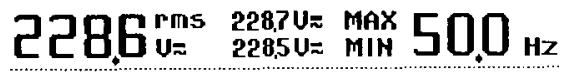

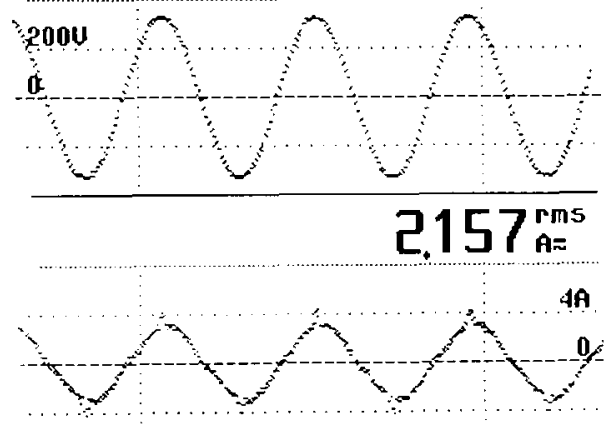

(b) Compensated Current

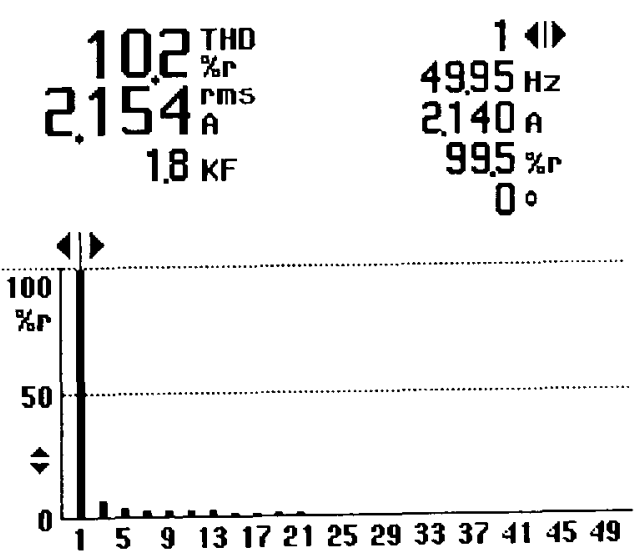

(c) Harmonic Spectrum

Fig.6.11: Loading condition for $\left(L_{m, H}=1 \mathrm{mH}\right)$ 


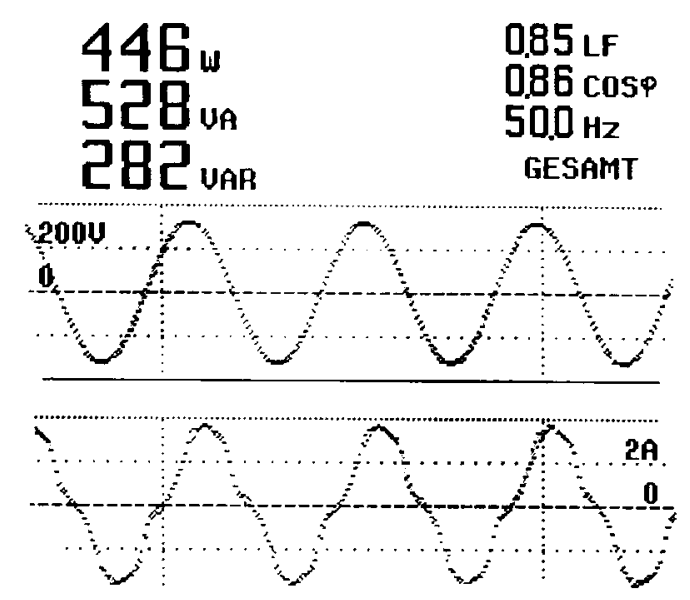

(a) Compensated Current (0.86 power factor)

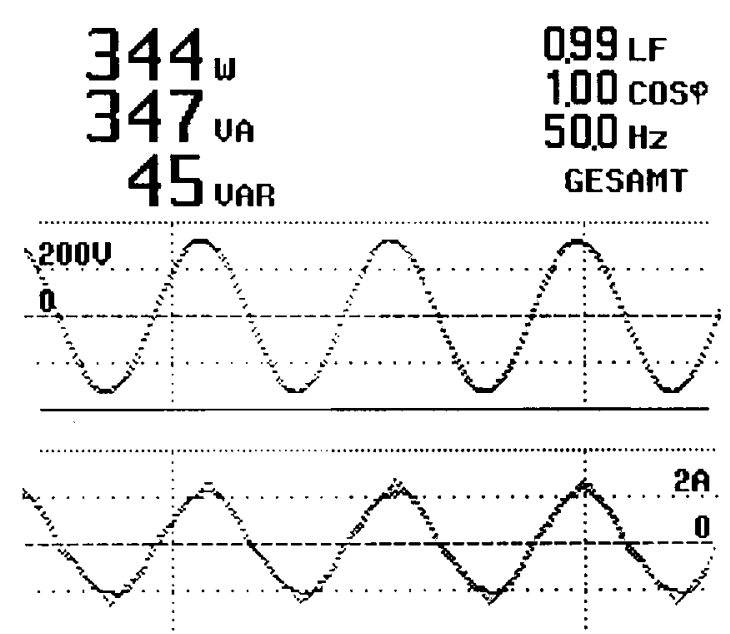

(b) Compensated Current (unity power factor)



(c) Harmonic Spectrum

Fig.6.12: Loading condition for power factor correction $\left(L_{\ldots .}=1 \mathrm{mH}\right)$ 


\subsection{Analysis of Losses}

Fig.6.13 and Fig.6.14 show the measured inverter losses dependent on the inverter inductance and switching frequency. Fig.6.13 indicates that the IGBT turn-off losses decrease and the turn-on losses increase with increasing inverter inductance and switching frequency. For lower switching frequency, the losses are lower (Fig.6.14). Conduction losses are constant for almost all values of inverter inductance.

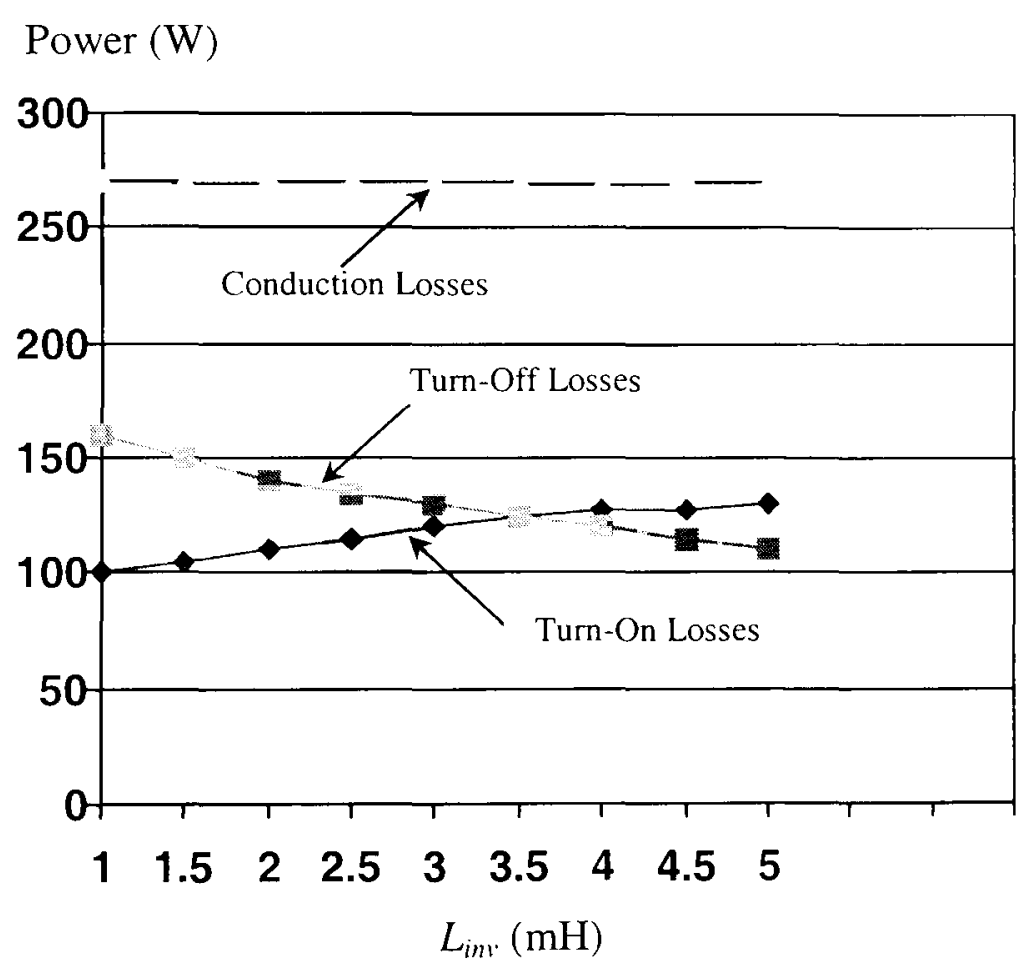

Fig.6.13: System Losses at $20 \mathrm{kHz}$ 


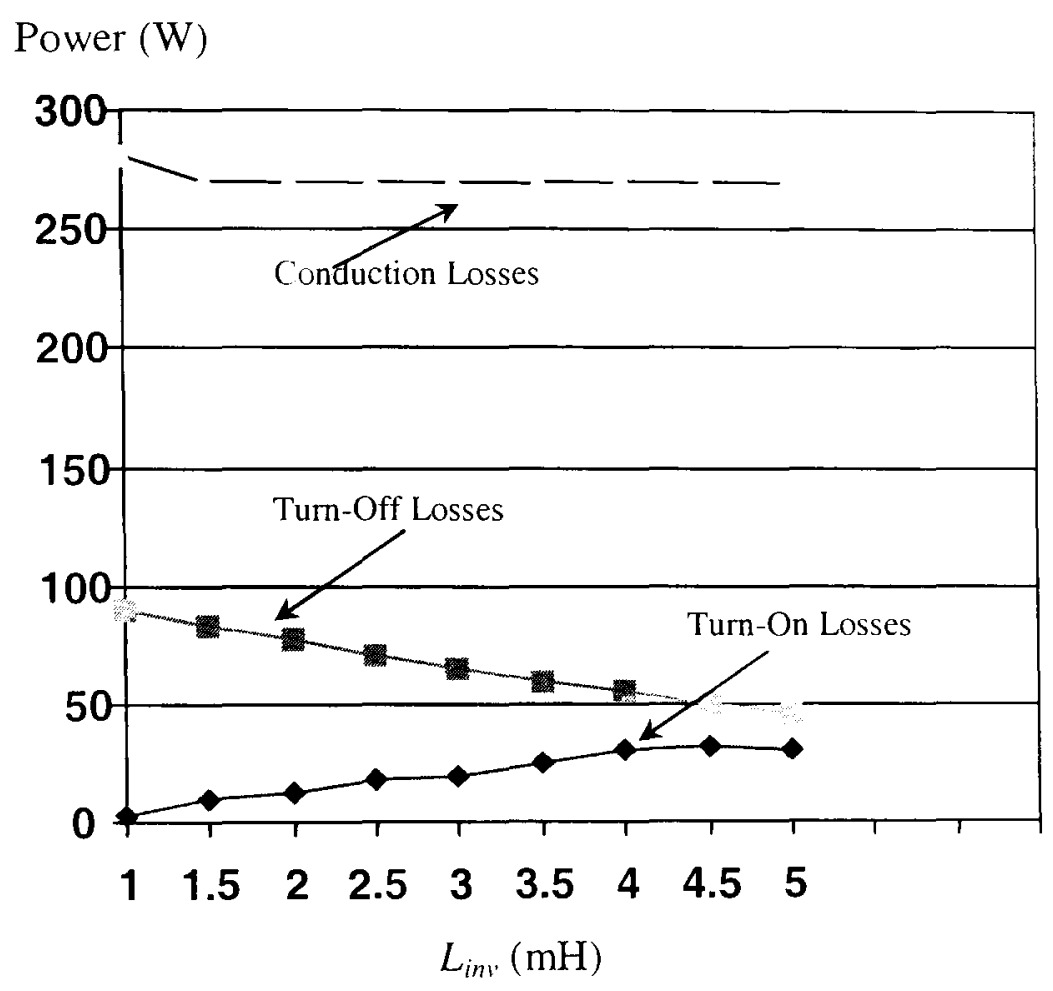

Fig.6.14: System Losses at $6 \mathrm{kHz}$

\subsection{Time Response}

The response time of the inverter was also measured. Compared to the existing power conditioner in the industry, the proposed circuit is very fast to compensate for the harmonics and reactive power. The response time for commercial industrial compensators from leading companies is in the range of $20-40 \mathrm{msec}$ for 40 Amp IGBTs. The proposed system has a time response of $1 \mathrm{msec}$ when used with a $1 \mathrm{mH}$ inverter inductance. This value is slightly increased to $3.5 \mathrm{msec}$ for the case of $4 \mathrm{mH}$ which is not acceptable from the point of view of performance (nonlinearities such as notches). The value of $1 \mathrm{~ms}$ is then the one attributed and the system can be stated to be at least 20 times faster than the commercial products from the leading manufacturers in the field of power systems and electronics. 


\subsection{Summary}

In this chapter, the construction of a single-phase configuration of the proposed active power filter has been described. The overall block diagram of the proposed system was successfully implemented in real industrial situations. The practical results presented here show that the shunt active power inverter with the passive filter combination successfully reduces unwanted harmonics generated by non-linear load. The filter can respond within $1 \mathrm{~ms}$ to rapidly varying load conditions. The proposed system can also reduce the electromagnetic interference in a range of $30 \mathrm{~dB} / \mathrm{div}$.

Due to the nature of the fast calculations being performed, inverter filters are mostly suited to industrial and commercial building applications which contain a significant number of computers and other non-linear loads; this type of filter is therefore highly suitable for future power quality problems. 


\section{Chapter 7}

\section{Conclusions and Future Work}

\subsection{General Conclusions}

A new method of power factor correction, harmonic reduction and load modelling for power systems using Insulated Gate Bipolar Transistors (IGBT) is presented in this thesis. Principles of operation, methods of control, modelling, analysis and design of filters are shown. Theoretical investigations were carried out to evaluate the performance of the proposed method. Although the problems associated with network disturbances and electrical pollution are being studied, there is still a need to find better and faster solutions.

Measurements prove that electronic components are the main source of harmonics in networks. The highest voltage level is measured for the fifth harmonic, which spreads over all voltage levels of the entire network independent of coupling to transformers. In medium-voltage mains, more and more consumers are being supplied directly. The power supply companies now request the installation of harmonics limits based on the user pays principle. In each specific case, the network must be analysed and investigated or technical and economical solutions in order to improve the power factor and to minimise harmonics. The results show that the method presented here contributes to the optimisation of efficiency and to avoid harmonics. 
An extensive survey of the most important publications relating to reactive power compensation and to harmonics was carried out and is summarised and discussed in chapter 2. Techniques to improve and correct the power factor have been brought up to date. In addition, reactions on the mains from non-linear loads have been discussed.

Active filtering and its control technique are discussed in great detail. A huge number of active power filter circuit configurations and compensation methods have been proposed in the literature. The control algorithm of active power filter circuits is very complex. In addition, the system impedance, which affects the magnitudes of the harmonics, is very dependent on loads and time, making it difficult to measure. The validity of this method has been verified by a software system, which was developed to evaluate real reactive power situations.

For this new method of harmonic current reduction, the filter circuit parameters were analysed, calculated and described. This filter configuration, which can be applied to single- or three-phase low power systems, was shown to provide an alternative approach to existing ones.

A software program is presented in this work which calculates harmonics in a power system. The network impedances were determined based on measurements at the point of common coupling (PCC). 


\subsection{Discussion of Results}

In this thesis, it has been demonstrated that a combination of IGBT-inverter with passive shunt filters connected to the inverter can be used to eliminate harmonics and to improve power quality. Furthermore, a new control method has been developed to increase the response time of the inverters and to reduce power losses.

This work can be seen as providing an alternative technique to those currently existing in the industry. The employment of the proposed technique for reactive power compensation offers the following benefits:

- $\quad$ exact compensation of power factor.

- continuous controllability.

maximum utilisation of capacitor capacity.

PC and Digital Signal Processor are not needed for the control unit.

The circuit presented in this thesis has only few electronic power components.

good compensation in keeping within reactive power and $\cos \varphi$ limits, even under varying reactive power demands

additional supply of active power to minimise load peaks and to support the network.

Further advantages are:

Switching can be used frequently.

Components are economically priced and widespread.

Large scale integration leads to low costs and good reliability in use.

- Standard application in power electronics is feasible. 
- Simple control concepts can be applied.

- $\quad$ Lower EMI emissions.

The modelling of the proposed system in both the time and frequency domain was presented in chapter 3. The presented block diagrams and the reduced transfer function model analysed in the s-domain show that the system is stable from the point of view of magnitude response up to high frequencies over $1 \mathrm{kHz}$. This frequency is equivalent to the $20^{\text {th }}$ harmonic of the load current. The compensation of the system at $20 \mathrm{kHz}$ switching frequency and the supply frequency of $50 \mathrm{~Hz}$ are very far from any resonance problems that may occur in the system. The characteristic equation of the open loop has the following roots:

$$
s_{I}=-68.5 \text { (dominant pole) and } s_{I}=-688610.5
$$

which are in the left hand side of the s-plane and the system is stable in the open loop and is able to compensate for the high order harmonics and to reduce their effect on the power system since it operates as a low pass filter with a minimum of $17 \mathrm{~dB}$ attenuation in all the cases.

The analytical and experimental results prove the validity of the proposed method. The simulation results show significant reduction of harmonics and correction of the power factor. The design can be implemented with commercially available components. The power factor is controlled in real time. Consequently, the system is suitable for applications which require a rapid effect on the power factor. The system can feed inductive and capacitive reactive power into the network. both highly dynamic and continuous. 
Various non-linear loads were simulated and tested using the dedicated simulation software. The system was shown to operate properly according to the design. From the measured data the complete low voltage and medium voltage power system can be simulated and designed.

The inverter losses depend on the inverter inductance and switching frequency. The IGBT turn-off losses decrease and the turn-on losses increase with increasing inverter inductance and switching frequency. For lower switching frequency, the losses are lower. Conduction losses are constant for almost all values of inverter inductance.

The power factor of the plant must be at least 0.95 . For this reason a compensation adjustment ( $\cos \varphi$-adjustment) was designed. Unity power factor was achieved by implementing the filter in the system. Different compensation degrees can also be achieved from the proposed system. This is very useful in the case of changing loads that need to be compensated for with continuous and smooth variation. In this case the proposed compensation can be used in conjunction with other switched static compensation techniques, generating stepped compensation levels. The exact full compensation of the system can be achieved using the proposed circuit. Moreover, several compensators can be used in parallel and the required compensation from each can be achieved using the controllable properties of the system as will be explained in the future works section.

The hysterisis control guaranties a stable operation for the complete system. The current control is necessary to ensure that the inverter follows exactly the required reference current. The achieved percentage errors were $\pm 5 \%- \pm 10 \%$ in some case depending on 
the value of the inverter inductance. For low value inductance it is clear that the current rate of change is much bigger than the case for higher values of inverter inductances. The inductance calculated from the theoretical equations of chapter 3 was $4 \mathrm{mH}$. However this value did not achieve the required compensation. This is mainly due to the fact that there are several non-linearities in the system which were neglected in the simulation. These non-linearities (magnetic circuits of the inductors and isolation transformers, switching delays, variations of the DC side capacitor voltage, ideal switches...) contribute together to make the inductor smaller $(1 \mathrm{mH})$. Both values were tested experimentally to decide the proper one to use. It turned out to be that the smaller value of inductance performs much better than the high value calculated from the equations.

The implemented PWM-hysterisis approach reduces the electromagnetic inteference. The inverter operates much better with the PWM hysterisis technique than without the PWM. This is manifested by several resonance effects, which occur for hysterisis circuits. The PWM also reduces the switching losses by ensuring that only one switch is turned on or off at a certain time. The fact also reduces the necessity for a higher switching frequency.

The operation of the proposed system was proven to be cost-effective when compensating for harmonics as well as for power factor. The fast time-response of the filter circuit is guaranteed to render the filter successful in fast changing harmonic compensation. The practical results presented in chapter 6 showed that the shunt active power filter with the passive filter combination successfully reduced unwanted harmonics generated by non-linear load as shown in Fig.6.8 and Fig.6.11. 
The filter can respond within $1 \mathrm{~ms}$ to rapidly varying load conditions. This response time of $1 \mathrm{~ms}$ is at least 20 times faster than commercial products from leading manufacturers in the field of power electronics and systems. This fact makes the proposed filter capable of compensating very fast changing harmonics generated by different loading conditions and dynamic loads in the power system.

Moreover, the proposed active shunt power circuit is at least 5 times cheaper and takes less volume than the available commercial products for the same load power. This is due to the efficient operation of the switches which reduces their switching losses and hence the overall temperature rise in the whole systems. The water-cooled switches proved to be more efficient in dissipating the heat generated from the switches, which implies that the forced air ventilation can be reduced and hence reduces the overall size of the filter.

\subsection{Practical Applications}

The shunt active and passive filter presented in this thesis has been designed and tested in a single-phase configuration. For each phase, the same printed circuit board is needed which is very easy and cheap to manufacture and to implement.

The circuit can be installed anywhere between the power source and the polluting equipment. By applying reactive power control, the system can improve the power factor and eliminate harmonics. The losses in the power circuit can be reduced hy applying passive filters. 


\subsection{Suggestions for Future Work}

This research has looked at the problems associated with low voltage and medium voltage distribution systems. The following work can be envisioned for the future:

- In this thesis, a single link in a low power systems were investigated from a practical point of view. Larger power systems have been left for further study and application development

- The performance of the proposed system could be improved from the point of view of the switching frequency and current error tracking by using more sophisticated control techniques. However, switching causes unavoidable voltage fluctuations and distortions, and thus reactions in other systems operated by the network

- The proposed system can be taken to investigate the transients, flicker, voltage sags and voltage swells.

- The controller and passive filter can be used in high power transmission lines to reduce harmonic reduction and voltage drop by connecting the proposed system in series with the power system.

- Balancing of the supply voltage and currents of the high power system can also be studied and investigated.

- The proposed complete system can be taken as a FACT-device to control long distances.

- For further research, to reduce the cost of implementation and to increase the performance of the proposed system, the control unit and other devices can be implemented in ASICs. 
- The efficiency of the complete system and reactions on the mains of different pollutions in conjunction with renewable energy sources, such as wind and solar energy, can be investigated

- As a further step, this proposed shunt active power filter with its different conditions and behaviours could be analysed and examined in a combination with series active filter and shunt passive filter and other configurations mentioned in Chapter 2 of this thesis 
References 


\section{List of References}

1. J.Arrillaga, B.C. Smith, N.R.Watson, A.R.Wood: "Power System Harmonic Analysis", John Wiley \&Sons, 1998

2. J.Arrillaga,, D.A.Bradly, P.S.Bodger: "Power System Harmonics", John Wiley \&Sons, 1985

3. T.J.E.Miller: "Reactive Power Control in Electric Systems", John Wiley \&Sons, 1982

4. W.K.Chen: "Passive and active Filters, Theory and Implementation", John Wiley \&Sons, 1986

5. R.C.Dungan, M.F.McGranaghan, "Electrical Power Systems Quality", Mc-Graw-Hill. 1996

6. Akagi H., "New trends in active filters", Proceedings of the EPE-95 Sevilla.

7. Grady W.M., Samotyj M.J., Noyola A.H., "Survey of active power line conditioning methodologies", IEEE-trans. on power delivery, Vol. 5, No. 3, July 1990.

8. Mehta P., Darwish M., Thomson T., "Active harmonic filters: an overview and comparison", in Proc. UPEC-89.

9. El-Habrouk M., Darwish M.K., Mehta P., "How to choose the appropriate active power filter for a specific application - Part 1: Power circuits and configurations, Part II: Compensated variables, control and reference estimation", MEPCON'97, January 1997, Alexandaria, Egypt.

10. VDEW: "Guide for public power supply networks", VDEW, 1992.

11. Xu W., Mansour Y., Siggers C., Hughes M.B., "Developing utility harmonic regulations based on IEEE STD-519-B.C. hydro's approach", IEEE-trans on Power Delivery, Vol. 10, No. 3, July 1995.

12. Krost, G.: "Frequenzabhängige Impedanzen von Verbrauchen Nieder- und Mittelspannunganetzen”. Universität Erlagen 1983. 
13. D.Nelles, L.Petry: "Wirk- und Blindleistungssteller zur Leistungssteuerung".. University of Kaiserlautern.

14. Gretsch, R. Dinkel, G.: "Kompensator für Oberschwingungen und Blindleistung". etz Archiv Bd. 9 (1987) H.1.

15. Working group : "A survey of North American electric utility concerns regarding nonsinusoidal waveforms". IEEE Transactions on Power Delivery, Vol.11,No.1, January 1996.

16. Working group: "Practical definitions for powers in Systems with non-sinusoidal waveforms and unbalanced loads: A discussion". IEEE Transactions on Power Delivery, Vol.11,No.1, January 1996.

17. R.Gretsch, G.Dinkel: "A New conditioner Parallel voltage source inverters for improvement of power quality", CIRED 1989.

18. H. Pouliquen, P. Lemerle, E. Plantive: "Voltage Harmonics Source Compensation Using a Shunt Active Filter", Sevilla, EPE 95.

19. J, Peschon et al.: "Optimum Control of Reactive Power Flow, IEEE Trans. Power Apparatus and System", Vol. PAS-87, Jan. 1968, pp. 40-48.

20. H. S. Patel, R.G. Hoft: "Generalised Techniques of Harmonic Elimination and Voltage Control in Thyristor Inverters Part I and II, IEEE Trans. on Industry Applications", Vol. IA-9, No. 3, May/June 1973.

21. Technischer Bericht: "Unregelmäßge Spannungsschwankungen in Drehstromnetzen Nr. 1-253", November 1983.

22. G.Lammer, A.Domijan: "Wattour Meter accuracy under controlled unbalanced harmonic voltage and current conditions". IEEE Transactions on Power Delivery, Vol. I 1,No.1, January 1996

23. R.Gretsch,Ch.Kuschnarew: "Parallel Voltage Source Inverters for Improvement of Power Quality, Power quality Bremen”, November 1995, Proceedings 211 
24. S. Civanlar, J.J. Grainger, "Volt/Var Control on Distribution Systems with Lateral Branches Using Shunt Capacitors and Voltage Regulators-II: The Solution Method", IEEE Trans. Power Apparatus \& Systems, Vol. PAS-104, Nov. 1985, pp. 3284-3290.

25. X. Dai, R. Gretsch: "Optimal Compensator Currents for the Reduction of the Harmonic Distortion in Networks. Part 1 and 2". ETEP Vol.4 No.4, July 1994.

26. S.M.Shahidehpour, N.I.Deeb: "An overview of the active power allocations in electric power systems". Electric Machines and Power Systems, 18:495-518, 1990.

27. Brauner, G.: "Berechnung der Oberschwingungsverteilung in Netzen". Tech. Mitt. AEG-Telefunken 71 (1981).

28. Gretsch, R., Stiller, T.: “Network disturbances caused by transient harmonics" IEE Conference Publication No.250 CIRED (1985), Part 1,S. 333-338.

29. "Spannungsqualität": Technischer Bericht Nr. 284, November 1995.

30. A. Cavallini, G.C. Montanari: "Compensation Strategies for Control of Shunt Active Filters". IEEE Transactions on Power electronics. Vol.9 No.6, November 1994.

31. J.K.Phipps, J.P.Nelson,P.K.Sen: "Power quality and harmonic distortion on distribution systems”. IEEE Transactions on Industry Applications, Vol.30,No.2,March/April 1994.

32. J.B. Ekanayake, N.Jenkins: "A three-level Advanced Var Compensator". IEEE Transactions on Power Delivery, Vol.11,No.1,January 1996.

33. Tuttas, C.: "Compensation of capacitive loads by a voltage-source active filter". ETEP Vol.2,No.1, January/February 1992.

34. T.Lequeu,M.Bensoam,C.Pahmer: "A new control method for active shunt harmonic current compensator". Power quality Bremen, November 1995, proceedings 221.

35. W.Mombacher, K.-H. Weck: FGH, "Load modelling for harmonic flow calculations". ETEP Vol.3,No.6,November/December 1993.

36. G. Choe, M. Park: "Analysis and Control of Active Power Filter with Optimised Injection", IEEE Trans. on Power Electronics, Vol. 4. No. 4. October 1989. 
37. P. Viriya, K. Matsuse: "Low-Harmonic GTO Converter for fundamental Power Factor Compensation". IEEE Transactions on Power Electronics. Vol.6 No.3, July 1991.

38. Marouchos C.C., "Switched capacitor circuits for reactive power generation", PhD thesis, Brunel University, UK, 1982.

39. Darwish M., "Switched capacitor filter for power applications", PhD thesis, Brunel University, UK, 1987.

40. Mehta P., Darwish M., Thomson T., "Switched capacitor filters", IEEE-Trans on Power Electronics, Vol. 5, No. 3, July 1990.

41. Y.H.Song, A.T.Johns, R.K.Aggarwal: "Nonlinear tyhristor-controlled var compensation". EPE, Brighton, 13-16/9/1993, University of Bath.

42. El-Habrouk M., Darwish M.K., Mehta P., "A new configuration for shunt active filter", EPE-97, Trondheim, Norway.

43. Enslin J., Van Wyk J.D., "Measurement and compensation of fictious power under nonsinusoidal voltage and current conditions", IEEE-trans on Instrumentation and Measurements, Vol. 37, No. 3, September 1988.

44. Enslin J., Van Wyk J.D., “A new control philosophy for power electronic converters as fictitious power compensators", IEEE-trans on Power Electronics, Vol. 8, No. 1, January 1990.

45. Van Harmelen G.L., Enslin J., "Real time dynamic control of dynamic power filters in supplies with high contamination", IEEE-trans on Power Electronics, Vol. 8, No. 3. July 1993.

46. Hsu, C.Y., Wu, H.Y., "A new single phase active power filter with reduced energy storage capacity", IEE proc. On electrical power applications, Vol. 143, No. 1, January 1996. 
47. Fujita, H, Akagi, H., Kohata, M., "Combined rectifying system including double series capacitor-smoothed diode rectifier and series active filter", in proceedings of IEE Japan, Vol. 120, No. 1, 1997.

48. Koozehkanani Z.D., Mehta P., Darwish M.K., "Active filter for eliminating current harmonics caused by non-linear circuit elements", IEE electronic letters, Vol. 31, No. 13, 22nd June 1995.

49. Koozehkanani Z.D., Mehta P., Darwish M.K., "Active Symmetrical lattice filter for harmonicc current reduction", EPE-95, Sevilla.

50. Koozehkanani Z.D., "Active filters: A unified Approach", PhD thesis, Brunel University, UK, 1996.

51. Koozehkanani Z.D., Mehta P., Darwish M.K., "An active filter for retrofit applications", in IEE-PEVD 1996.

52. Masaaki Ohshima, Eisuke Masada: "Novel error tracking mode ac current waveform control method and its ac filter design procedure", Sevilla EPE 95, page 2.472-2.477.

53. Kim, S., Park, J., Choe, G., Park, M., “An improved PWM current control method for harmonic elimination using active power filter", IEEE-IAS 1987.

54. Fukuda, S., Yamaji, M., "Design and characteristics of active power filter using current source converter", in IEEE-IAS 1990.

55. Viriya, P., Matsuse, K., "Low harmonic GTO converter for fundamental power factor compensation", IEEE-trans. on power electronics, Vol. 6, No. 3, July 1991.

56. Fukuda, S., Endoh, T., "Control method for a combined active system employing a current source converter and a high pass filter", IEEE-trans. on industry applications. Vol. 31, No. 3, May/June 1995.

57. Malesani, L., Rossetto, L., Tenti, P., "Active power filter with hybrid energy storige" IEEE-trans. on power electronics, Vol. 6, No. 3, July 1991. 
58. Furuhashi, T., Okuma, S., Uchikawa, Y., "A study on the theory of instantaneous reactive power", IEEE-trans. on industrial electronics, Vol. 37, No. 1, February 1990.

59. Moran L.A., Dixon J.W., Wallace R.R., "A three phase active power filter operating with fixed switching frequency for reactive power and current harmonic compensation", IEEE-trans. on Industrial Electronics, Vol. 42, No. 4, August 1995.

60. Hosseini, S.H., Tarafdar-Haque, M., "'The presentation of a new active filter with amplitude modulation of difference wave", proceedings of PEMC 94, Warsaw, Poland.

61. Wiliams, S.M., Hoft, R.G., "Adaptive frequency domain control of PWM switched power line conditioner", IEEE-trans. on power electronics, Vol. 6, No. 4, October 1991.

62. Bird, B.M., March, J.F., Mclellan P.R., "Harmonic reduction in multiplex converter by triple frequency current injection", IEE-Proc., Vol. 116, No. 10, 1969.

63. Inarida, S., Miyake, W., Mizuguchi, N., Hariama, T., Hoshi, K., "Development of three level power converter system using IGBTs for Sinkansen trains”, in proceedings of EPE 97, Trondheim, Norway.

64. Üouliquen, H., Lemerle, P., Plantive, E., "Voltage harmonics source compensation using a a shunt active filter", in proc. of EPE-95, Sevilla.

65. Karshenas, H.R., Kojori, H.A., Dewan, S.B., "Gereralized techniques of selective harmonic elimination and current control in current source inverters/converters", IEEEtrans. on power electronics, Vol. 10, No. 5, September 1995.

66. Diana, G., Chathury, A.S., Harley, R.G., Woodward, D.R., "Two quqdrant fully diditally controlled unity power factor converter", in proc. PEMC-94, Warsaw, Poland.

67. Koczara, W., Bialoskarski, P., "Modified rectifiers, with unity power factor", in proc, PEMC-94, Warsaw, Poland.

68. Shirahanma, H.-, Sakurai, Y., Matsuda, Y., Ishigaki, Y., Murai. K., "Inatantaneous control method with a GTO converter for active and reactive power in superconducting 
magnetic energy storage", IEEE trans. on power electronics, Vol. 9, No. 1, January 1994.

69. Thomson, T.-, Mehta, P., Darwish, Mostafa, "A novel reactive filter for DC ripple suppression".

70. Deib, D., Kim, J., "Nonlinear high-ftequency switching filters for the dc-side of ac/dc power converters", in proceedings of IEEE Industriel application society annual meeting 1996.

71. Bhavaraju, V.B., Enjeti, P., "A fast active power filter to correct line voltage sags", IEEE trans. on industrial electronics, Vol. 41, No. 3, June 1994.

72. Cho, G.C., Jung, G.H., Choi, N.S., Cho, G.H., "Analysis and controller design of static VAR compensator using three level GTO inverter", IEEE trans. on power electronics, Vol. 11, No. 1, January 1996.

73. Ooi, B., Dai, S., "Series type solid state static VAR compensator", IEEE trans. on power electronics, Vol. (, No. 2, April 1993.

74. Blajszczak, G., "Direct method for voltage distortion compensation in power networks by series converter filter", IEE-Proc. in Electrical power applications, Vol. 142, No. 5 , September 1995.

75. Peng, F.Z., Akagi, H., Nabae, A., "Compensation characteristics of the combined system of shunt passive and series active filters", IEEE-trans. on industry applications, Vol. 29, No. 1, January/February 1992.

76. Bhavaraju, V.B., Enjeti, P.N., "Analysis and design of an active power filter for balancing unbalanced loads", IEEE-trans. on power electronics, Vol. 8, No. 4. October 1993.

77. Cavallini A., Montanari G.C., "Compensation strategies for shunt active filter control". IEEE-trans on Power Electronics, Vol. 9, No. 6, November 1994. 
78. Jou, H.L., "Performance comparsion of the three phase active power filter algorithms". IEE-proc. Gereration, transmission and distribution, Vol. 142, No. 6, Nov. 1995.

79. Rodriguez, J., Wiechmann, E., Suarez, A., Barria, A., "A contribution to the control of a single phase active power filter", in proc. COBEP-93, Brasil.

80. Torry, D.A., Al-Zamel, M.A.M., "Single phase active power filters for multiple nonlinear loads", IEEE trans. on power electronics, Vol. 10, No. 3, May 1995.

81. Machmoum, M., Bruyant, N., Siala, S., Le Doeuff, R., "A practical approach to harmonic current compensation by single phase active filter", in proc. EPE-95, Sevilla.

82. Nastran, J., Cajhen, R., Seliger, M., Jereb, P., "Active power filter for nonlinear ac loads", IEEE trans. on power electronics, Vol. 9, No. 1, January 1994.

83. Choe, G., Park, M., "Analysis and control of active power filter with optimized injection", IEEE trans. on power electronics, Vol. 4, No. 4, October 1989.

84. Hayashi, Y., Sato, N., Takahashi, K., "A novel control of a current source active filter for ac power system harmonic compensation", IEE-trans. on industry applications, Vol. 27, No. 2, March/April 1991.

85. Joos, G., Moran, L., Ziogas, P., "Performance analysis of a PWM inverter VAR Compensator", IEEE Trans. on power electronics, Vol. 6, No. 3, July 1991.

86. Saetieo, S., Devaraj, R., Torrey, D.A., "The design and implementation of a three phase active power filter based on sliding mode control", IEEE-trans. on industry applications, Vol. 31, No. 5, September/October 1995.

87. Edwich, G., Doulai, P., "Multi converter performance and active filtering", IEEE-trans. on power electronics, Vol. 10, No. 3, May 1995.

88. Blajszczak, G., "Static VAR compensator with fully controlled reactors", in IEE Proc. on electrical power applications, Vol. 141, No. 5, September 1994. 
89. Verdelho, P., "Space vector based current controller in $\alpha \beta 0$ coordinate system for the PWM voltage converter connected to the AC mains", in proceedings of Power electronics specialists conference, PESC-97.

90. Bhattacharya, S., Divan, D., "Synchronous frame based controller implementation for a hybrid series active filter system", IEEE-Industrial application society annual meeting IAS-95.

91. Bhattacharya, S., Veltman, A., Divan, D., Lorenz, R. "Flux based active filter controller", IEEE-Industrial application society annual meeting IAS-95.

92. De Doncker, R.W., Vandenput, J.A., "A two dimensional FFT algorithm for three phase inverter fed systems", IEEE-trans. on power electronics, Vol. 7, No. 1, January 1992.

93. Luop, S., Hou, Z., "An adaptive detecting method for harmonic and reactive currents", IEEE-trans. on industrial electronics, Vol. 42, No. 1, February 1995.

94. Aburto, V., Schneider, M., Moran, L., Dixon, J., “An active power filter implemented with a three level NPC voltage source inverter", in proceedings of Power Electronics Specialists Conference 1997.

95. Fujita, H., Akagi, H., "The unified power quality conditioner: the integration of series active filters and shunt active filters", in proceedings of Power Electronics Specialists Conference 1996.

96. Libano, F., Simonetti, D., Uceda, J., "Frequency characteristics of hybrid filter systems", in proceedings of Power Electronics Specialists Conference 1996.

97. Mannon, G., Delarue, P., Bausiere, R., Crappe, M., Reglet, M., Dupuis, S., "Design of a unified power flow controller for implementation into a micronetwork", in proceedings of EPE-97, Trondkeim, Norway.

98. Rastogi, M., Mohan, N., Edris, A., "Hybrid-active filtering of harmonic currents in power systems", IEEE-trans. on power delivery, Vol. 10, No. 4. October 1995. 
99. S.Bernard, G.Trochan: "A new high performance active harmonic conditioner based on the current injection mode". Power quality Bremen. November 1995 Proceedings $2+3$.

100. M.-X. Wang, H.Pouliquen: "Performance of an active filter using PWM current source inverter". The European Power electronics association, 1993,P218-223.

101. Chicharo, J.F., Wang, H., "Power system harmonic signal estimation and retrieval for active power filter applications", IEEE-trans. on power electronics, Vol. 9, No. 6 . November 1994.

102. Brod, D.M., Novotny, D.W., "Current control of VSI-PWM inverters", IEEE-Industry Applications, Vol. IA-21, No. 4, May7June 1985.

103. Höfner, J. Aredes, M., Heumann, K., "Utilization of a small rated shunt active power filter combined with a conventional passive filter for large power systems", in Proc. PEMC-94, Warsow, Poland.

104. Harley, R.G., Rigby, B.S., Jennings, G.D., "Design of a controlled converter which emulates a series capacitive compensator for long power lines", in Proc. PEMC-94, Warsaw, Poland.

105. H.W. Dommel, W.F. Tinney, "Optimal Power Flow Solutions", IEEE Trans. Power Apparatus \& Systems, Vol. PAS-87, Oct. 1968, pp. 1866-1876

106. J. Peschon et al., "Optimum Control of Reactive Power Flow", IEEE Trans. Power Apparatus \& Systems, Vol. PAS-87, Jan. 1968, pp. 40-48.

107. W.R. Barcelo et al., "Optimization of the Real-Time Dispatch With Constraints for Secure Operation of Bulk Power Systems", IEEE Trans. Power Apparatus \& Systems, Vol. PAS-96, May/June 1977, pp. 741-757.

108. D.I. Sun et al., "Optimal Power Flow by Newton Approach", IEEE Trans. Power Apparatus \& Systems, Vol. PAS-103, Oct. 1984, pp. 2864-2880.

109. R.R. Shoults, D.T. Sun, “Optimal Power Flow Based Upon P-Q Decomposition”. IEEE Trans. Power Apparatus \& Systems, Vol. PAS-101. Feb. 1982, pp. 397-405. 
110. N. Flatabo et. al., "Transformer Tap Setting in Optimal Lead Flow", IEEE Trans. Power Apparatus \& Systems, Vol. PAS-104, June 1985, pp. 1356-1362.

111. S. Civanlar, J.J. Grainger, "Volt/Var Control on Distribution Systems with Lateral Branches Using Shunt Capacitors and Voltage Regulators-I: The Overall Problem“, IEEE Trans. Power Apparatus \& Systems, Vol. PAS-104, Nov. 1985, pp. 3278-3283.

112. S. Civanlar, J.J. Grainger, "Volt/Var Control on Distribution Systems with Lateral Branches Using Shunt Capacitors and Voltage Regulators-III: The Numerical Results”, IEEE Trans. Power Apparatus \& Systems, Vol. PAS-104, Nov. 1985, pp. 3291-3297.

113. R.C. Burchatt et al., "Developments in Optimal Power Flow", IEEE Trans. Power Apparatus \& Systems, Vol. PAS-101, Feb. 1982, pp. 406-414.

114. R.C. Burchatt et al., "Large Scale Optimal Power Flow", IEEE Trans. Power Apparatus \& Systems, Vol. PAS-101, Oct. 1982, pp. 3722-3732.

115. C. Burchatt et al., "Quadratically Convergent Optimal Power Flow", IEEE Trans. Power Apparatus \& Systems, Vol. PAS-103, Nov. 1984, pp. 3267-3275.

116. M.O. Mansour, T.M. Abdel-Rahman, "Non-Linear VAR Optimization Using Decomposition and Coordination", IEEE Trans. Power Apparatus \& Systems, Vol. PAS-103, Feb. 1984, pp. 246-255.

117. I. Hano et al., "Real Time Control of System Voltage and Reactive Power", IEEE Trans. Power Apparatus \& Systems, Vol. PAS-88, Oct. 1969, pp. 1544-1559.

118. S. Narita, M.A. Hamman, "Multicomputer Control of System Voltage and Reactive Power on Real-Time Basis", IEEE Trans. Power Apparatus \& Systems, Vol. PAS-92, Jan./Feb. 1973, pp. 278-286.

119. R.R. Shoults, M.S. Chen, "Reactive Power Control by Least Squares Minimization", IEEE Trans. Power Apparatus \& Systems, Vol. PAS-95, Jan./Feb. 1976, pp. 325-334. 
120. C. Savulescu, "Qualitative Indices for the System Voltage and Reactive Power Control", IEEE Trans. Power Apparatus \& Systems, Vol. PAS-95, July./Aug. 1976, pp. 1413-1421.

121. Eric Hobson, "Network Constained Reactive Power Control Using Linear Programming", IEEE Trans. Power Apparatus \& Systems, Vol. PAS-99, May/June 1980, pp. 868-877.

122. K.R.C. Mamandur, R.D. Chenoweth, "Optimal Control of Reactive Power Flow for Improvements in Voltage Profiles and for Real Power Losses Minimization”, IEEE Trans. Power Apparatus \& Systems, Vol. PAS-100, July 1981, pp. 3185-3193.

123. W.O. Stadlin, P.L. Fletcher, "Voltage Versus Reactive Current Model for Dispatch and Control”, IEEE Trans. Power Apparatus \& Systems, Vol. PAS-101, Oct. 1982, pp. $3751-3758$.

124. S. Rama lyer et al., "New Technique for Optimal Reactive-Power Allocation for Loss Minimization in Power System", IEE Proceedings, 1983, pp. 178-182.

125. S. Elangovan, "New Approach for Real Power Loss Minimization", IEE Proceedings, Nov. 1983, pp. 295-299.

126. K.Y. Lee et al., “A United Approach to Optimal Real Reactive Power Dispatch”, IEEE Trans. Power Apparatus \& Systems, Vol. PAS-104, May. 1985, pp. 1147-1153.

127. S.M. Shahidehpour, J. Qiu, “A New Approach for Minimizing Power Losses and Improving Voltage Profile", IEEE Trans. Power Apparatus \& Systems, Vol. PAS-106, May. 1987 , pp. 267-295.

128. N. Deeb and S.M. Shahidehpour, "An Efficient Technique for Reactive Power Dispatch Using a Revised Linear Programming Approach", Electric Power Systems Research Journal, Vol. 15, No. 1, 1988, pp. 121-134. 
129. N. Deeb and S.M. Shahidehpour, "Linear Reactive Power Optimization in a Large Power Network Using the Decomposition Approach", IEEE Transactions on Power Systems, Vol. 5, No. 2, May 1990.

130. N. Deeb and S.M. Shahidehpour, "Optimal Decentralized Control of the Voltage Profile in a Large Scale Power Network", Submitted to Automatica Journal.

131. N. Deeb and S.M. Shahidehpour, "Improved System Operation by Optimal Adjustment of Reactive Generation in Electric Power Networks", International Journal of Energy Research, 1990.

132. K. Iba et al., "Practical Reactive Power Allocation/Operation Planninng Using Successive Linear Programming", IEEE Trans. on Power Systems, Vol. 3, No. 2, pp. $558-566,1988$.

133. R. Fernandes et al., "Large Scale Reactive Power Planning" IEEE Trans. Power Apparatus \& Systems, Vol. PAS-102, May 1983, pp. 1083-1088.

134. S. Granville et al., "An Integrated Methodology for VAR Sources Planning”, IEEE Trans. on Power Systems, Vol. 3, No. 2, pp. 549-557, 1988.

135. S. Rama lyer, K. Ramachandran and s. Hariharan, "Optimal Reactive Power Allocation for Improved System Performance", IEEE Trans. Power Apparatus \& System, PAS103, June 1984, pp. 1509-1515.

136. K. Aoki, W. Fan and A. Nishikori, "Optimal VAR Planning by Approximation Method for Recursive Mixed-Integer Linear Programming", IEEE Trans. Power Apparatus \& System, Vol. 3, No. 4, pp. 1741-1747, 1988.

137. N. Deeb and S.M. Shahidehpour, "Economic Allocation of Reactive Power Supply in an Electric Power Network", Proceedings of the IEEE International Symposium on Circuits and Systems, pp. 1859-1862, May 1989.

138. N. Deeb and S.M. Shahidehpour, "Adecomposition Approach for Minimizing Real Power Losses in Power Systems", Proceedings of IEE, Part C, 1990. 
139. N. Deeb and S.M. Shahidehpour, "Cost Minimization in Allocation of Reactive Power Sources", Electrical Power and Energy Systems Journal, 1990.

140. K.H. Abdul-Rahman, S.M.Shahidehpour,M.Danesdoost: "AI Approach to optimal VAR control with Fuzzy reactive power", IEEE Transactions on power systems, Vol. 10,No.1 Febr.1995.

141. H. Abdul-Rahman, S.M.Shahidehpour: "A fuzzy based optimal reactive power control", IEEE Transactions on power systems, Vol. 8,No.2, May 1993.

142. K.H. Abdul-Rahman, S.M.Shahidehpour: "Reactive power optimisation using fuzzy load representation", IEEE Transactions on power systems, Vol. 9,No.2, May 1994.

143. K.H. Abdul-Rahman, S.M.Shahidehpour: "Application of fuzzy sets to optimal reactive power planning with security constraints", IEEE Transactions on power systems, Vol. 9,No.2, May 1993.

144. Y.L.Chen,C.C.Liu: "Interactive fuzzy satisfying method to optimal multi-objective VAR planning in power systems", IEE Proc. Gen, Transm. Distr, Vol.141.No.6 Nov. 1994.

145. P.K.Dash,S,Mishra,A.C.Liew. "Fuzzy logic based VAR stabiliser for power system control", IEE Proc. Gen, Transm. Distr, Vol.142.No.6 Nov.1995.

146. B.K.Bose: "Expert systems, Fuzzy logic and neutral network applications in power electronics and motion control", Proceedings of the IEEE, Vol.82, No.8,Aug. 1994.

147. R.K.Montana, G.G.Richards: "Harmonic source monitoring and identification using neutral networks", IEEE Transactions on power systems, Vol. 5,No.4, Nov. 1990.

148. S.Osowski: "Neutral network for estimation of harmonic components in power system". IEE Proceedings-C, Vol.139, No.2, March 1992.

149. H.Mori,K.Itou,H.Uematsu,S.Tsuzuki: "An artificial Neutral-based method for predicting power system voltage harmonics", IEEE Transactions on Power Delivery. Vol.7. No.1, January 1992. 
150. Gretsch, R, Krost, G: "Measurement and calcutation of harmonic impendances of loads, LV and MV networks", CIRED 1983, Liege, c.02.

151. "Harmonics: Characteristic parameters, methods of study, estimates of existing values in the network",. Electra 77(1982)35-54.

152. Stiller, T.: "Ein Beitrag zur Bestimmung der Oberschingungen in elektrischen Niederund Mittelspanunngsnetzen".

153. Gretsch, R., Gunselmann, W.: "Oberschwingungen in Nieder- und Mittelspannungsnetzen - Analyse der Störstromquellen und Maßnahmen zur Störunterdrückung”.

154. FGH: "Oberschwingungsgehalt und Netzimpedanzen elektrischer Nieder- und Mittelspannungsnetze”, FGH, Technischer Bericht 1-268, 1988.

155. Gretsch : Gretsch,R. Krost,G. "Einfluß von Verbrauchern auf die frequenzabhängige Netzimpedanzen", etz a 4(1982).

156. Filipski P.S., “Comments on „Measurement and compensation of fictitious power under nonsinusoidal voltage and current conditions", IEEE-trans on Instrumentation and Measurements, Vol. 38, No. 3, June 1989.

157. Czarnecki L.S., “Comments on „Mesurement and compensation of fictitious power under nonsinusoidal voltage and current conditions", IEEE-trans on Instrumentation and Measurements, Vol. 38, No. 3, June 1989.

158. Czarnecki L.S., Comments on "A new control philosophy for power electronic converters as fictitious power compensator", IEEE-trans on Power Electronics, Vol. 5, No. 4, October 1990.

159. "Semicron Innovation and Service Catalogue", 1999 
Appendices 


\section{Appendix A}

Control Block diagrams of the Transfer Functions of the Filter 


\section{Control Block Diagrams of the Transfer Functions of the Filter}

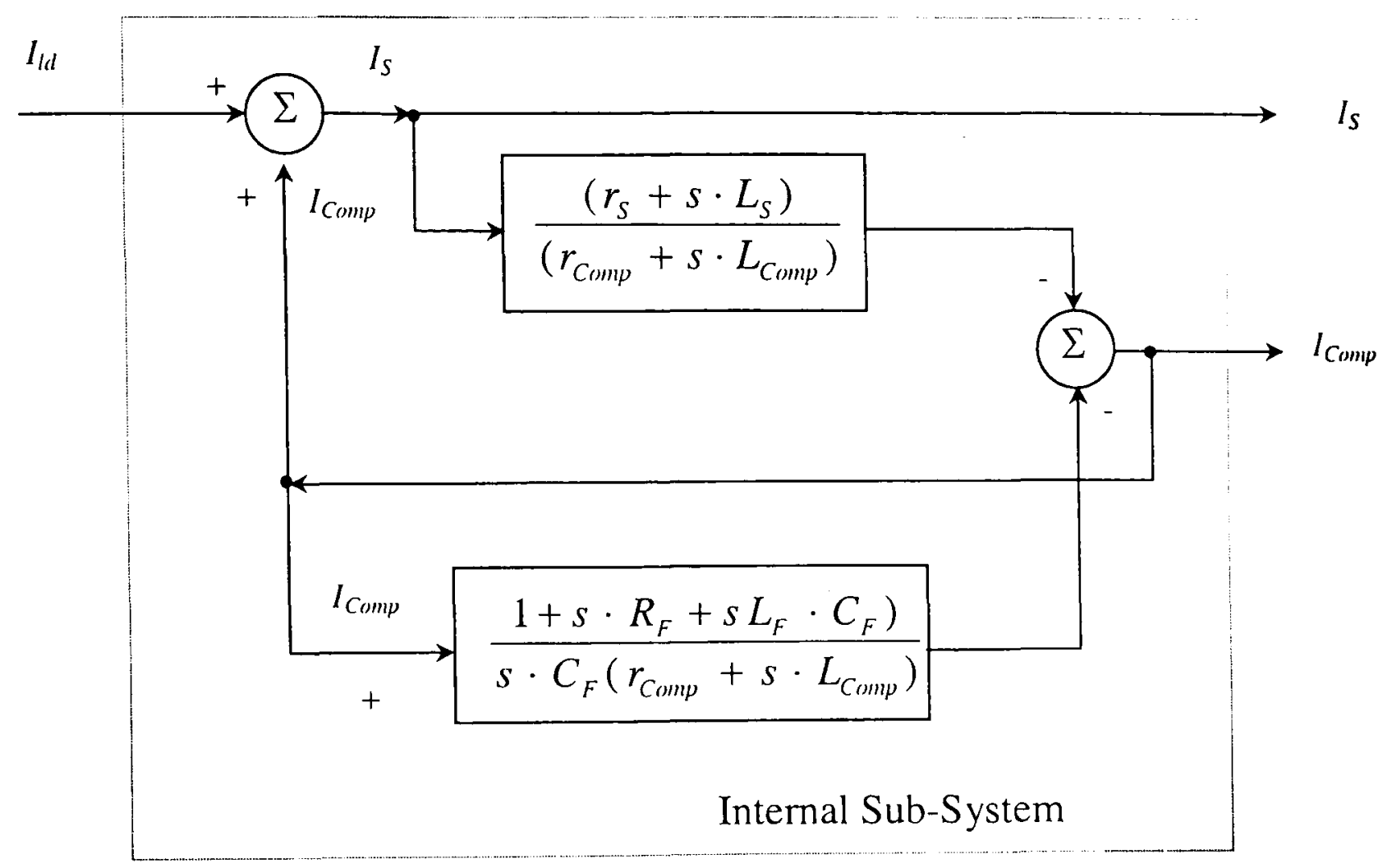

Fig.A1. Control Block Diagram of $G_{11}(s)$

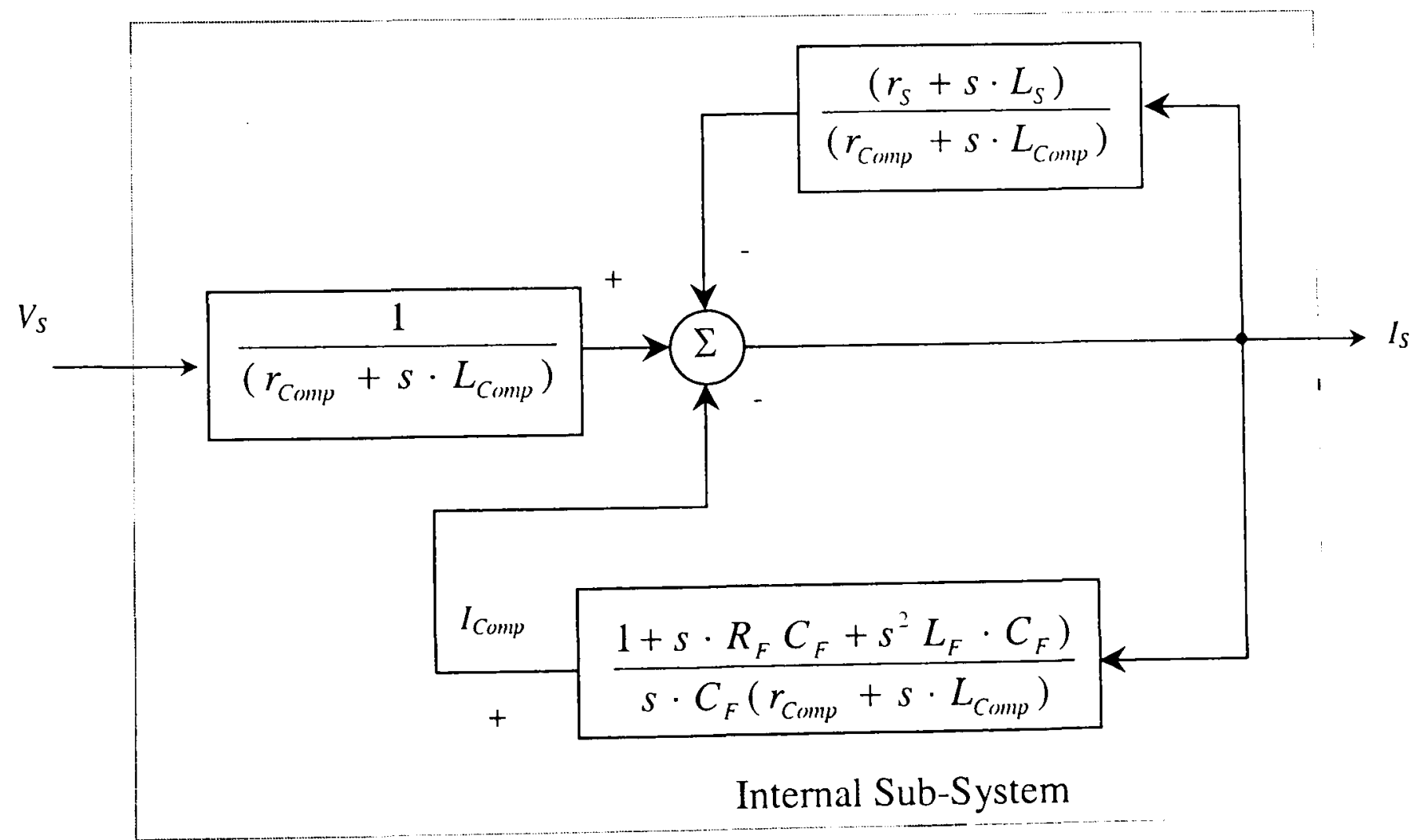

Fig.A2: Control Block Diagram of $G_{12}(s)$ 


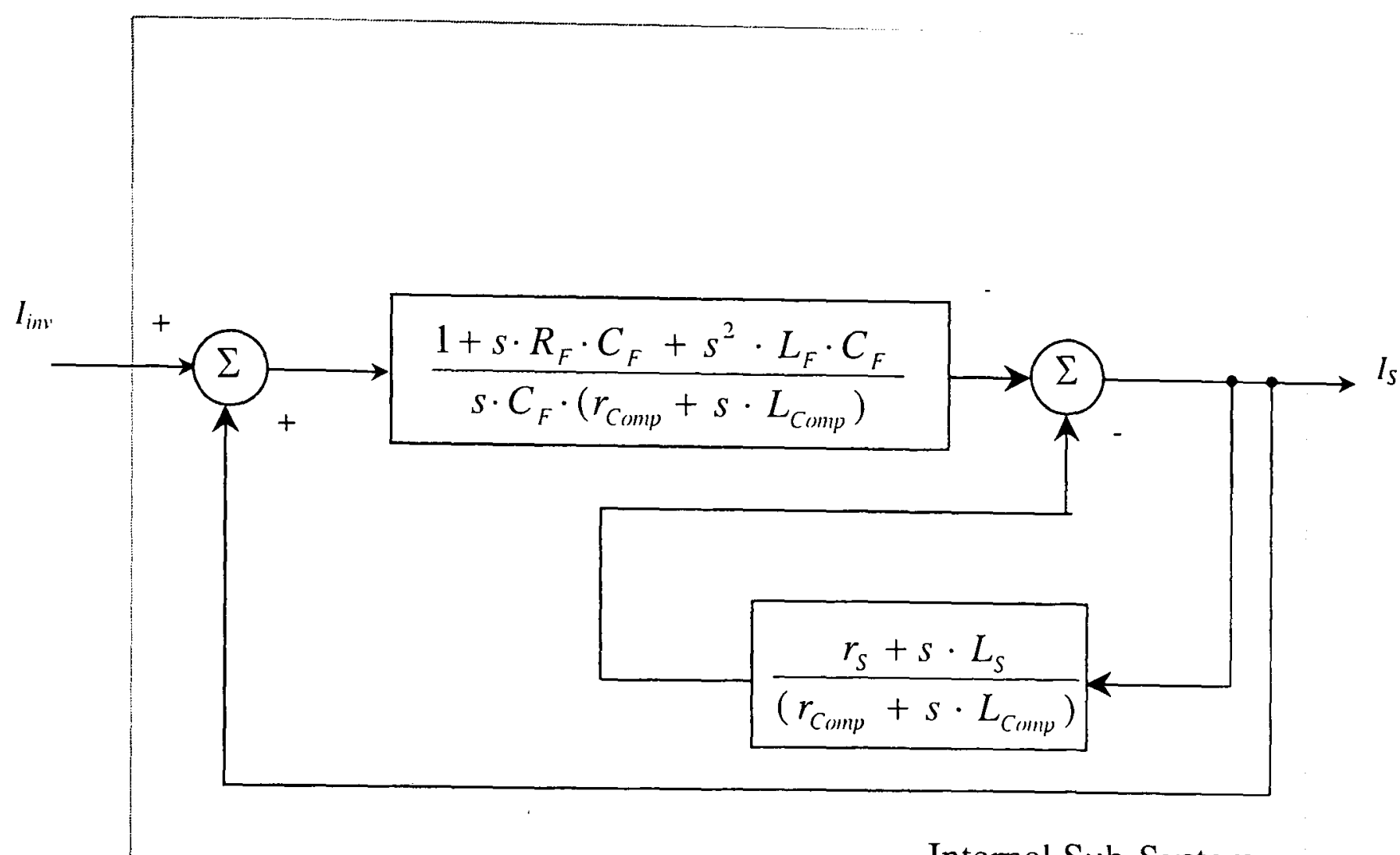

Internal Sub-System

Fig.A3: Control Block Diagram of $G_{13}(s)$

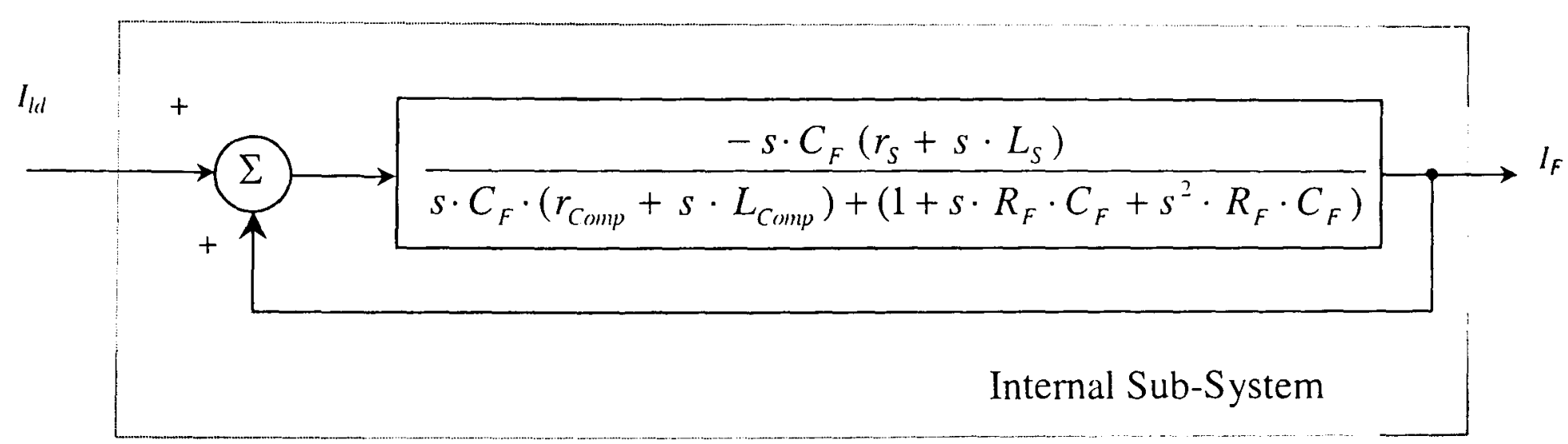

Fig.A4: Control Block Diagram of $G_{2 I}(s)$ 


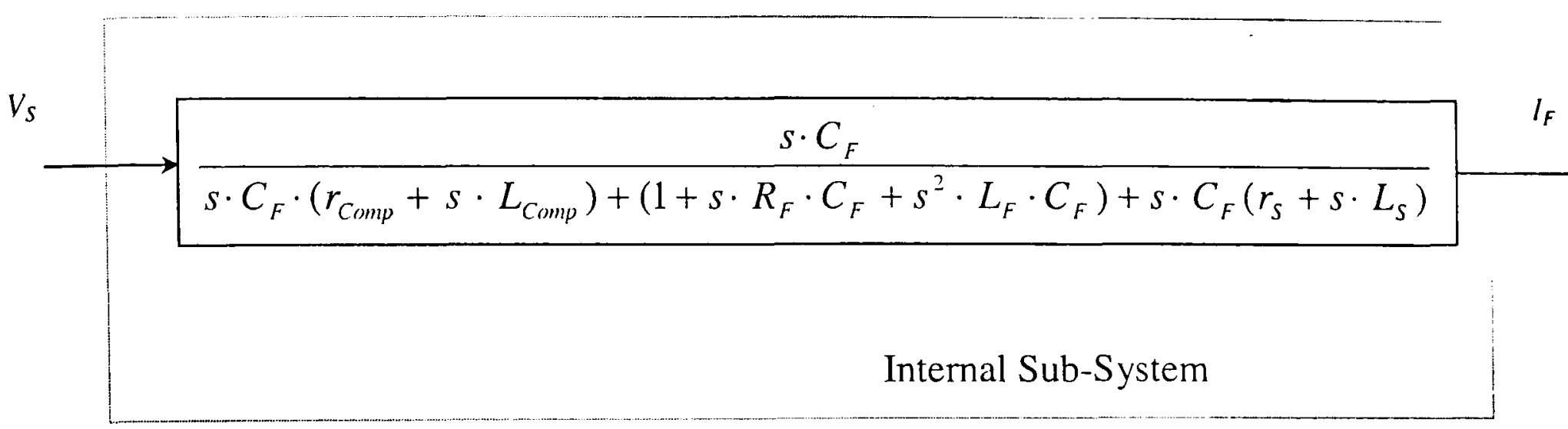

Fig.A5: Control Block Diagram of $G_{22}(s)$

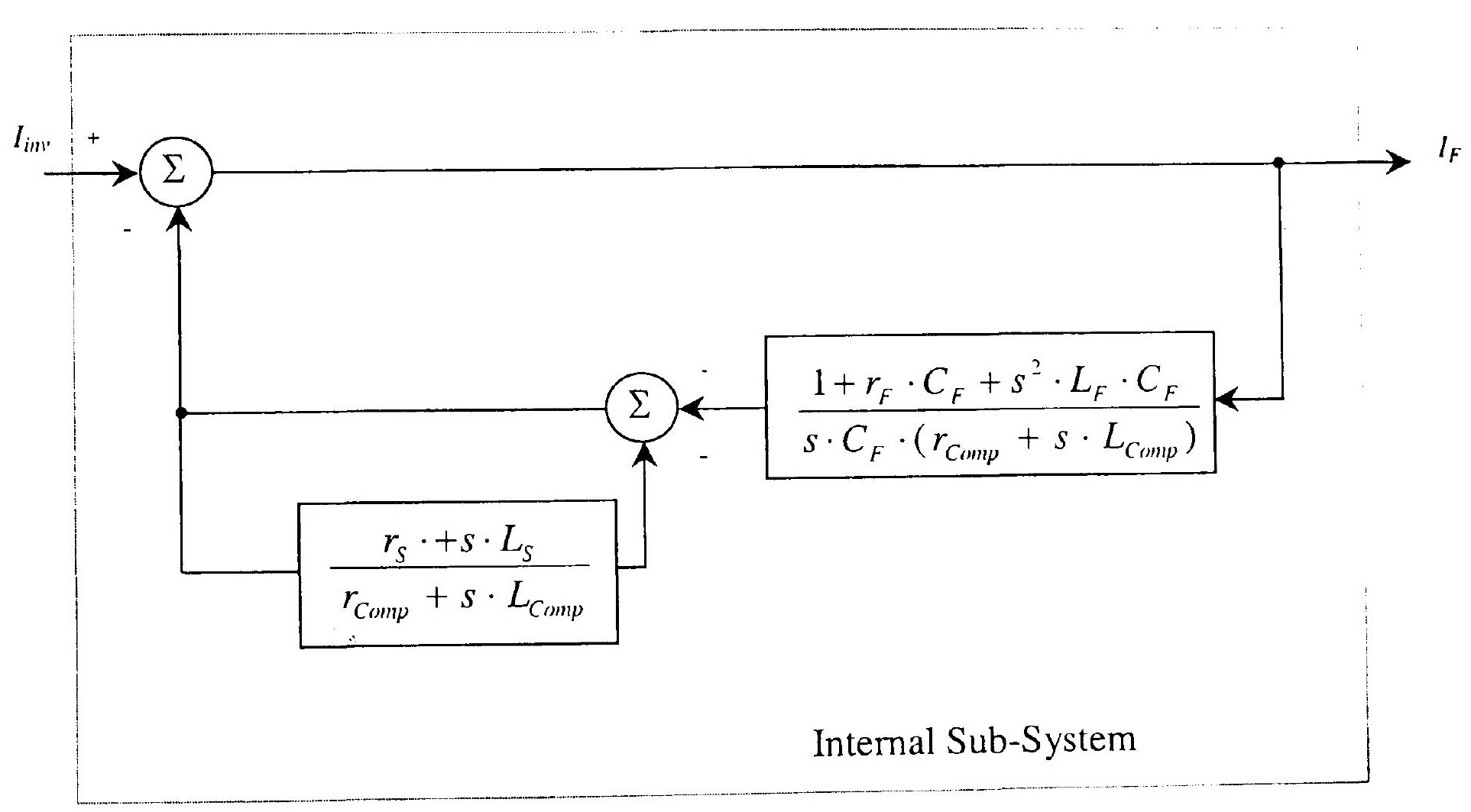

Fig.A6: Control Block Diagram of $G_{23}(s)$ 


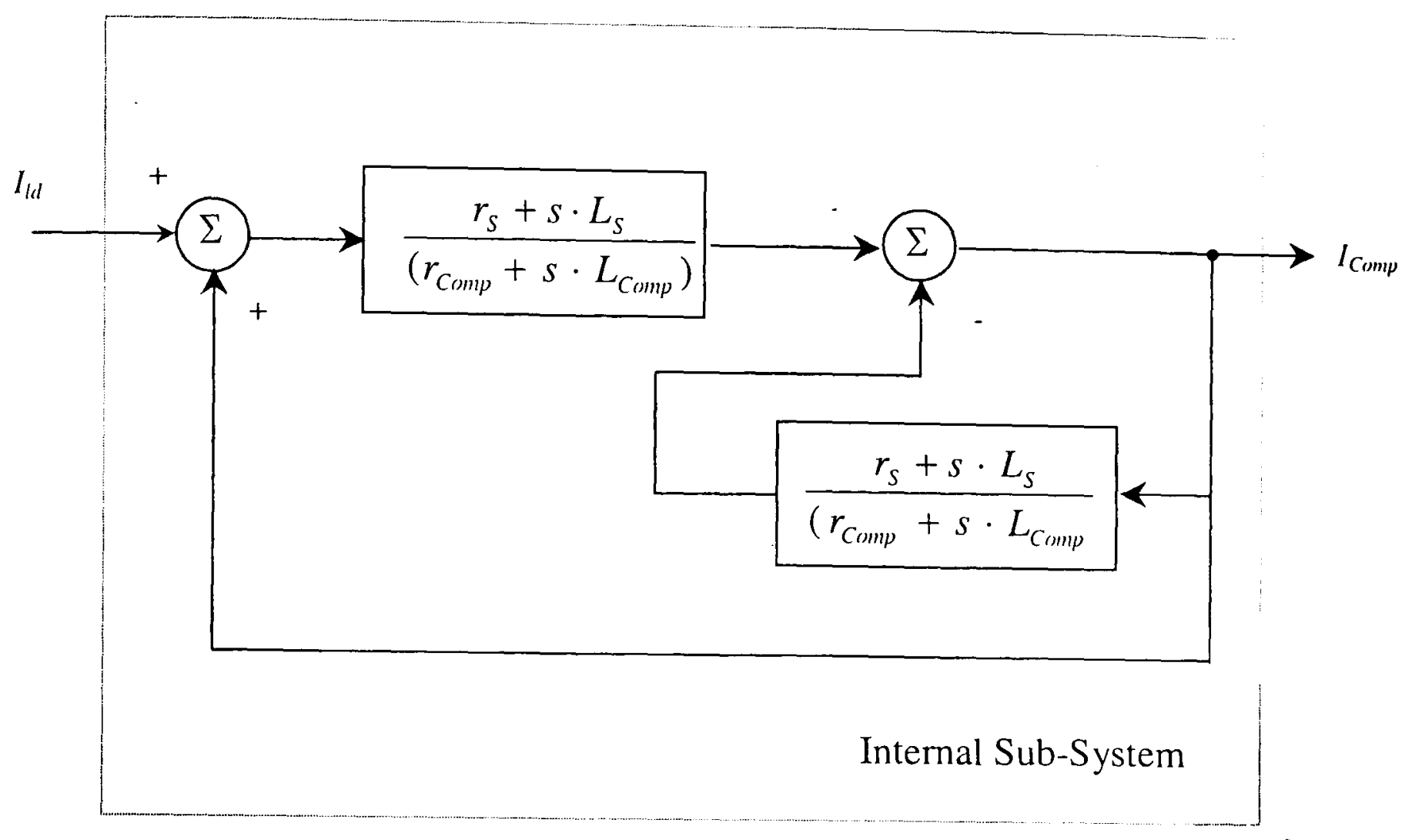

Fig.A7: Control Block Diagram of $G_{3 l}(s)$

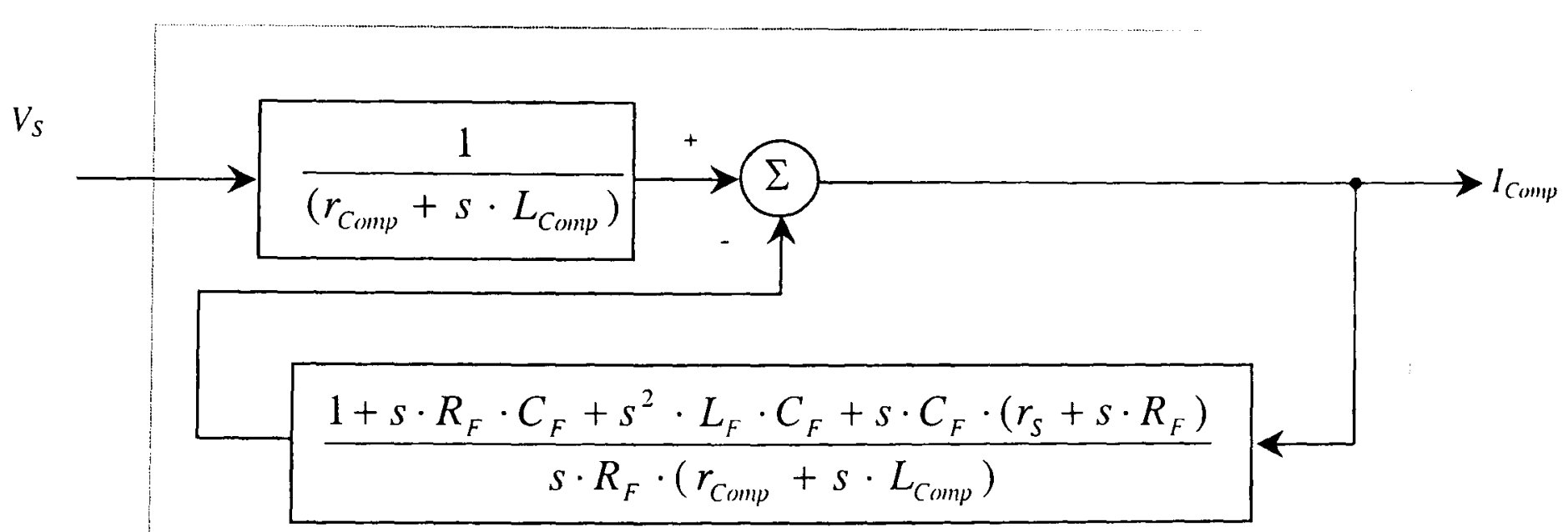

Internal Sub-System

Fig.A8: Control Block Diagram of $G_{32}(s)$ 


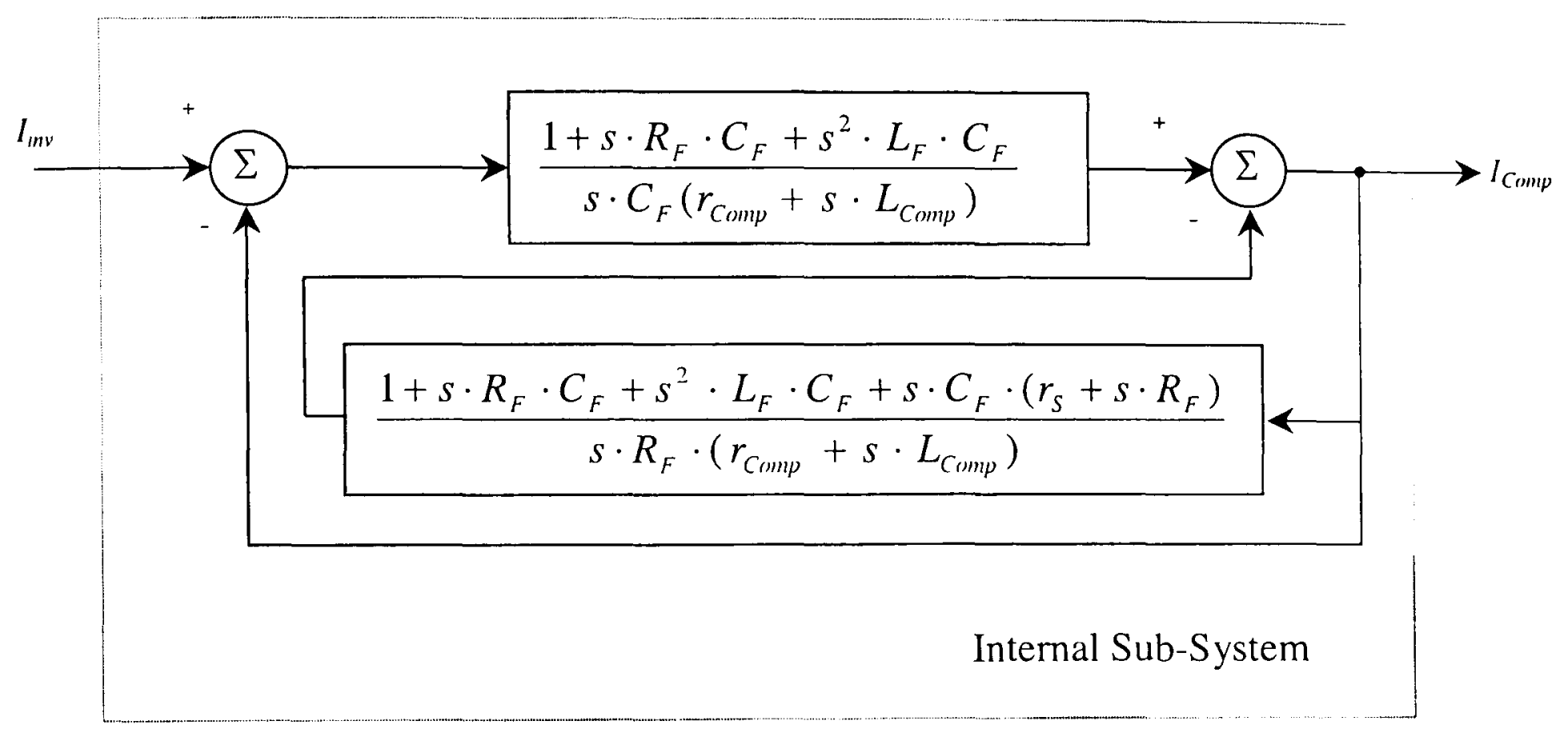

Fig.A9: Control Block Diagram of $G_{3,3}(s)$ 


\section{Appendix B}

Parameters for the Inverter and Filter Circuits 


\section{Inverter characteristics}

$$
\begin{aligned}
& V_{D C}>V_{n} \cdot \sqrt{2} \cdot 120 \% \\
& V_{B l o c k \text { king }}>V_{D r} \cdot 150 \% \cdots 200 \% \\
& i_{I G B T}>2 \cdot i_{L \max } \\
& L_{\text {inverter }}>\frac{V_{D C}}{f_{S} \cdot \Delta i_{\text {max }}}
\end{aligned}
$$

\section{Filter characteristics}

$$
C_{F}=10 \mu F
$$

$$
R_{F}=1.12 \Omega
$$$$
L_{i n,}=4 m H
$$

$$
T_{s}=20 \mu s
$$

$$
T_{D}=9 \mu s
$$

$$
f_{s}>20 \cdot f_{\text {, }}
$$

\section{Load characteristics}

Non-linear loads with different power ratings 


\section{Appendix C}

Software Program for the Evaluation of the Proposed Control System 
* Reactive Power Compensation and Harmonic Simulation

*

* IGBT-Inverter anf Filter Circuit

maclib:'p:'peclsystem.pml'

- Characteristics of the IGBT

real, table :: pswitchingt (10)! Total switching losses

- real, table :: Vce (8) ! Voltage-Current Characteristics of the IGBT

- real, table :: ufwd (8) ! Voltage-Current Characteristics of the Diode

- Individual losses

* Conducting losses

real :: plt1, plt2, plt3, plt 4

! IGBT losses

real :: pld1, pld2, pld3, pld4 ! Diode losses

real :: ptotal

! Total losses of all semi-conductors

- Switching losses

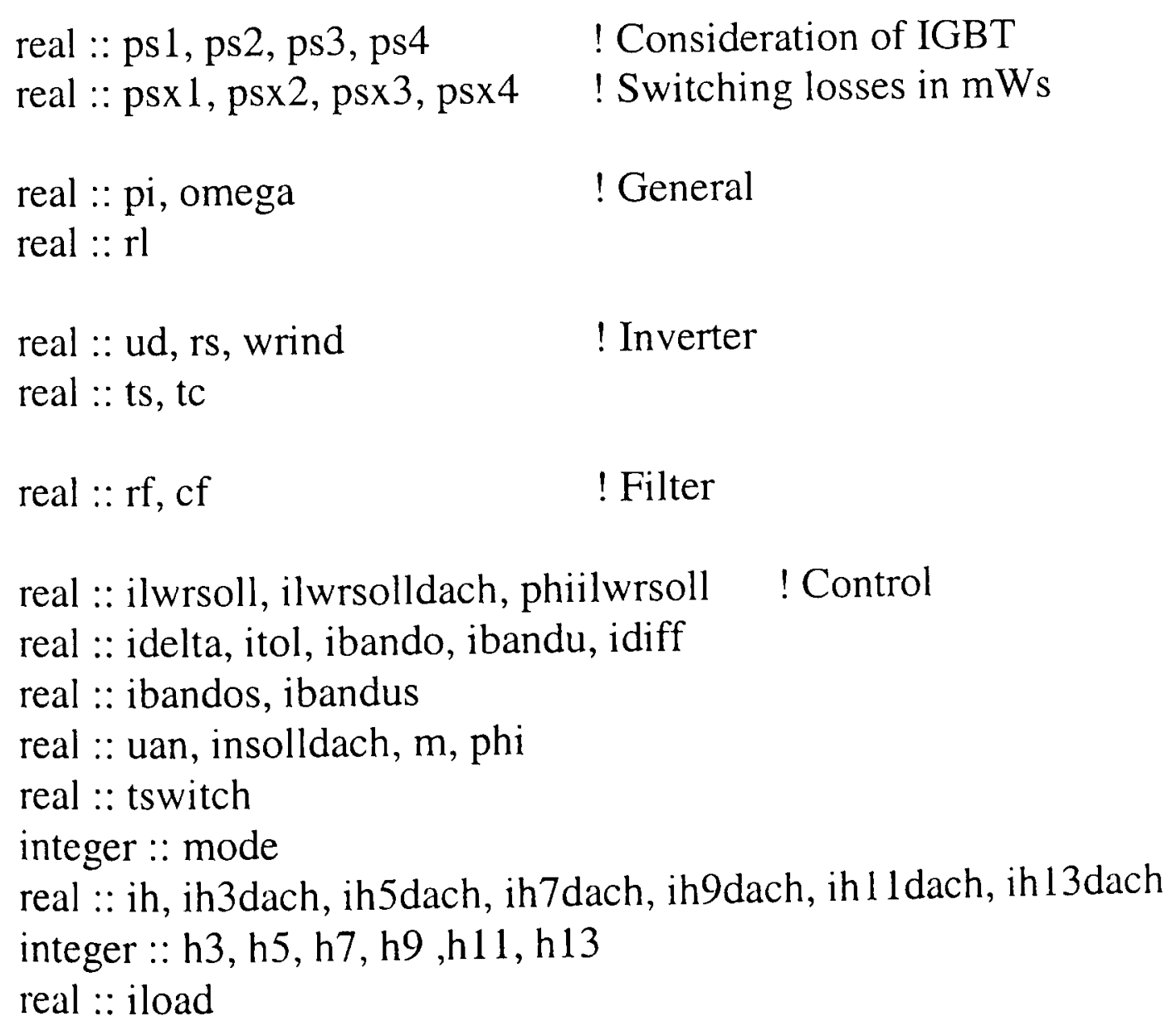



real $:: \mathrm{fn}$, undach, $\ln , \mathrm{rn}$
! Network
real $::$ rv 1, lv 1
! Consumption

$* \operatorname{misc}$

real $::$ muell

initial!

muell $=0$.

iload $=0$.

plt $1=0$.

plt2 $=0$.

plt3 $=0$.

plt4 $=0$.

pld $1=0$.

pld2 $=0$.

pld3 $=0$.

pld $4=0$.

ps $1=0$.

ps $2=0$.

ps3 $=0$.

ps $4=0$.

$\operatorname{psx} 1=0$.

$\operatorname{psx} 2=0$.

$\mathrm{ps} \times 3=0$.

psx4 $=0$.

psumme $=0$.

$\mathrm{fn}=50$.

! Network

undach $=325$.

$\ln =0.65 \mathrm{e}-3$

$\mathrm{rn}=0.2723$

$\mathrm{rv} \mathrm{l}=10$.

$\operatorname{lv} 1=.02387$

ih $=0$.

$\mathrm{pi}=2 . * \operatorname{acos}(0$.

! General

omega $=2{ }^{*}{ }^{\mathrm{p} i}{ }^{*} \mathrm{fn}$

$\mathrm{rl}=1 . \mathrm{e}-6$ 


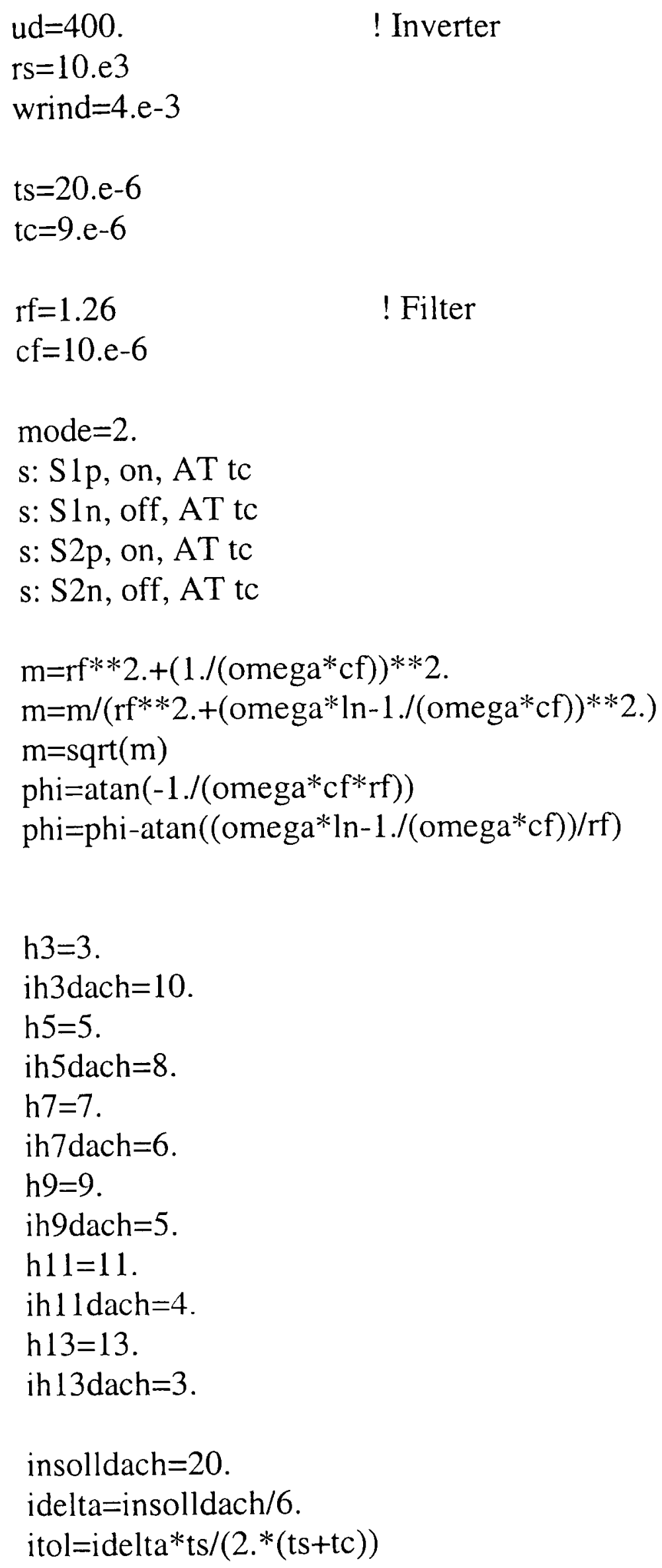


- Characteristic Vce $=\mathrm{f}(\mathrm{Ic})$ for turned-on IGBT

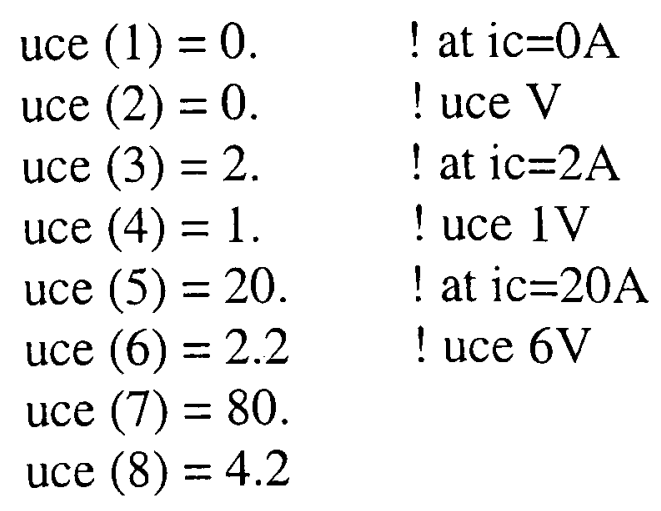

- Characteristic Vfwd $=\mathrm{f}(\mathrm{Ic})$ for conducting Diode

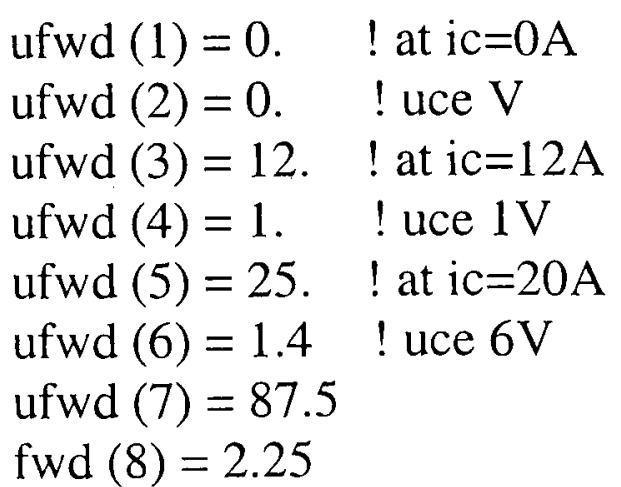

- $\quad$ Characteristics $P s=f(I c)$

$$
\begin{aligned}
& \text { ps }(1)=0 \\
& \text { ps }(2)=0 \\
& \text { ps }(3)=25 . \\
& \text { ps }(4)=5.5 \\
& \text { ps }(5)=40 . \\
& \text { ps }(6)=10 . \\
& \text { ps }(7)=60 . \\
& \text { ps }(8)=18 . \\
& \text { ps }(9)=80 . \\
& \operatorname{ps}(10)=24 .
\end{aligned}
$$

activate: scanning_iLinverter, all ts

* activate: jumping, at 0.015

endsection!-

network ! 
b: Vgl (WR1,WR0), e=ud, r=rl ! Inverter

b: S1p (WR1,WRx), rsw=rs, r=rl, semi=GTO, NONC

b: Vlp (WRx,WR1), rsw=rs, r=rl, semi=DIODE, NONC

b: $\operatorname{Sin}(\mathrm{WRx}, \mathrm{WR} 0), \mathrm{rsw}=\mathrm{rs}, \mathrm{r}=\mathrm{rl}$, semi=GTO, NONC

b: V $\ln ($ WR0,WRx), rsw=rs, r=rl, semi=DIODE, NONC

b: S2p (WR 1,0), rsw=rs, r=rl, semi=GTO, NONC

b: V2p (0,WR1), rsw=rs, r=rl, semi=DIODE, NONC

b: S2n (0,WR0), rsw=rs, r=rl, semi=GTO, NONC

b: V2n (WR0,0), rsw=rs, r=rl, semi=DIODE, NONC

b: LWR (WRx, 1), l=wrind

b: ZNET $(1, N x), l=\ln , r=r n$

! Network

b: UNET $(\mathrm{Nx}, 0), \mathrm{e}=$ undach* $^{*} \sin$ (omega*time)

b: RFIL $(1, F x), r=r f$

! Filter

b: CFIL (Fx,0), c=cf

b: IH3 $(1,0), q=$ ih3 dach* $\sin ($ omega*h3*time)

! i-Harmonics

b: IH5 $(1,0), q=i h 5$ dach* $\sin ($ omega $*$ h5*time)

b: IH7 $(1,0), q=i h 7$ dach $^{*} \sin ($ omega*h $7 *$ time)

b: IH9 $(1,0), q=i h 9$ dach* $\sin ($ omega*h $9 *$ time)

b: IH1 $1(1,0), q=$ ih 11 dach* $\sin ($ omega*h $11 *$ time)

b: IH13 $(1,0), q=$ ih 13 dach* $\sin ($ omega*h $13 *$ time)

endsection!

*event: jumping!-

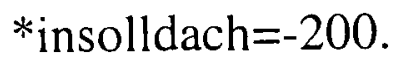

idelta $=$ insolldach $/ 6$.

$*$ itol=idelta*ts $/(2 *(\mathrm{ts}+\mathrm{tc}))$

endsection!

event: scanning_iLWR

tswitch $=$ time+tc

idiff $=$ i_LWR-ilwrsoll 
if (idiff .lt. -itol) then

if (mode .eq. 3 ) then

s: SIn, off, AT tswitch

psx $2=p s 2$

s: Slp, on, AT tswitch

psx $1=0$.

mode $=2$.

elseif (mode .eq. 2) then

s: S2p, off, AT tswitch

ps $x 3=p s 3$

s: S2n, on, AT tswitch

psx $4=0$.

mode $=1$.

Endif

elseif (abs(idiff) .le. itol) then

if (mode .eq. 1) then

s: S2n, off, AT tswitch

psx $4=$ ps 4

s: S2p, on, AT tswitch

$\operatorname{ps} \times 3=0$.

mode $=2$.

elseif (mode .eq. 3) then

s: SIn, off, AT tswitch

ps $22=p s 2$

s: Slp, on, AT tswitch

psx $1=0$.

mode $=2$.

endif

elseif (idiff .gt. itol) then

if (mode .eq. 1) then

s: S2n, off, AT tswitch

ps $x 4=p s 4$

s: S2p, on, AT tswitch

ps $\times 3=0$.

mode $=2$.

elseif (mode .eq. 2) then

s: SIp, off, AT tswitch

psx $1=p s 1$

s: SIn, on, AT tswitch

ps $2=0$.

mode $=3$.

endif

endif

endsection 
System Calculations !

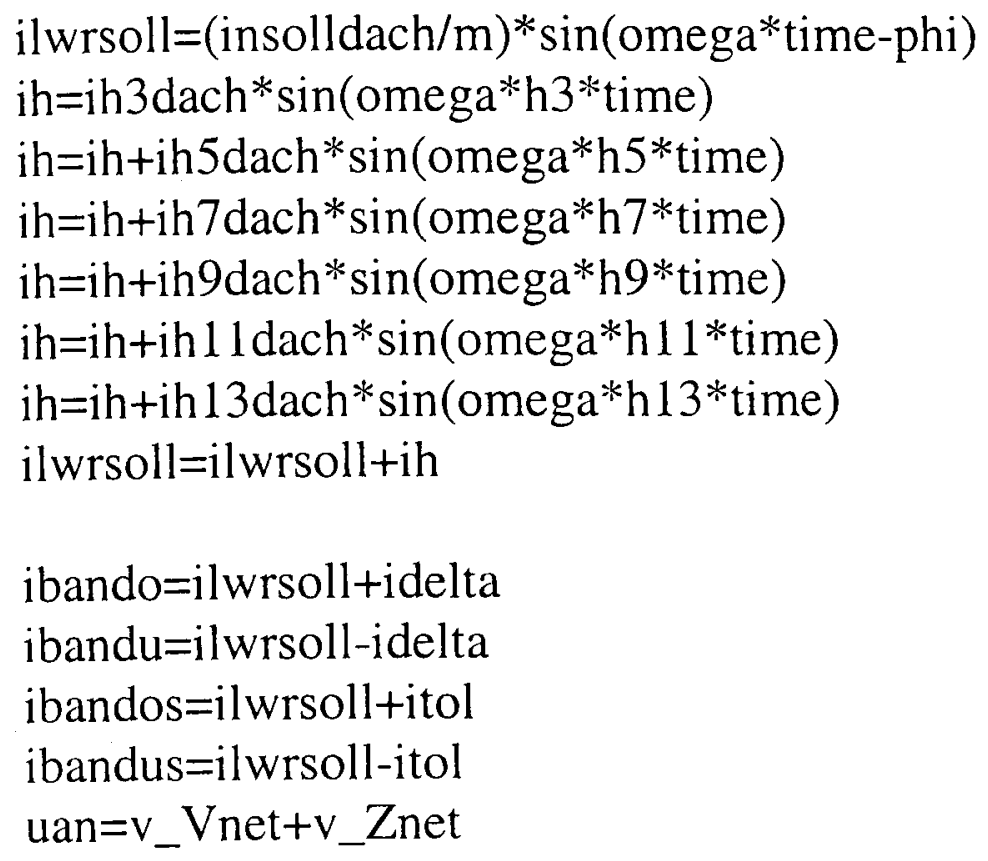

- Calculation of conducting losses

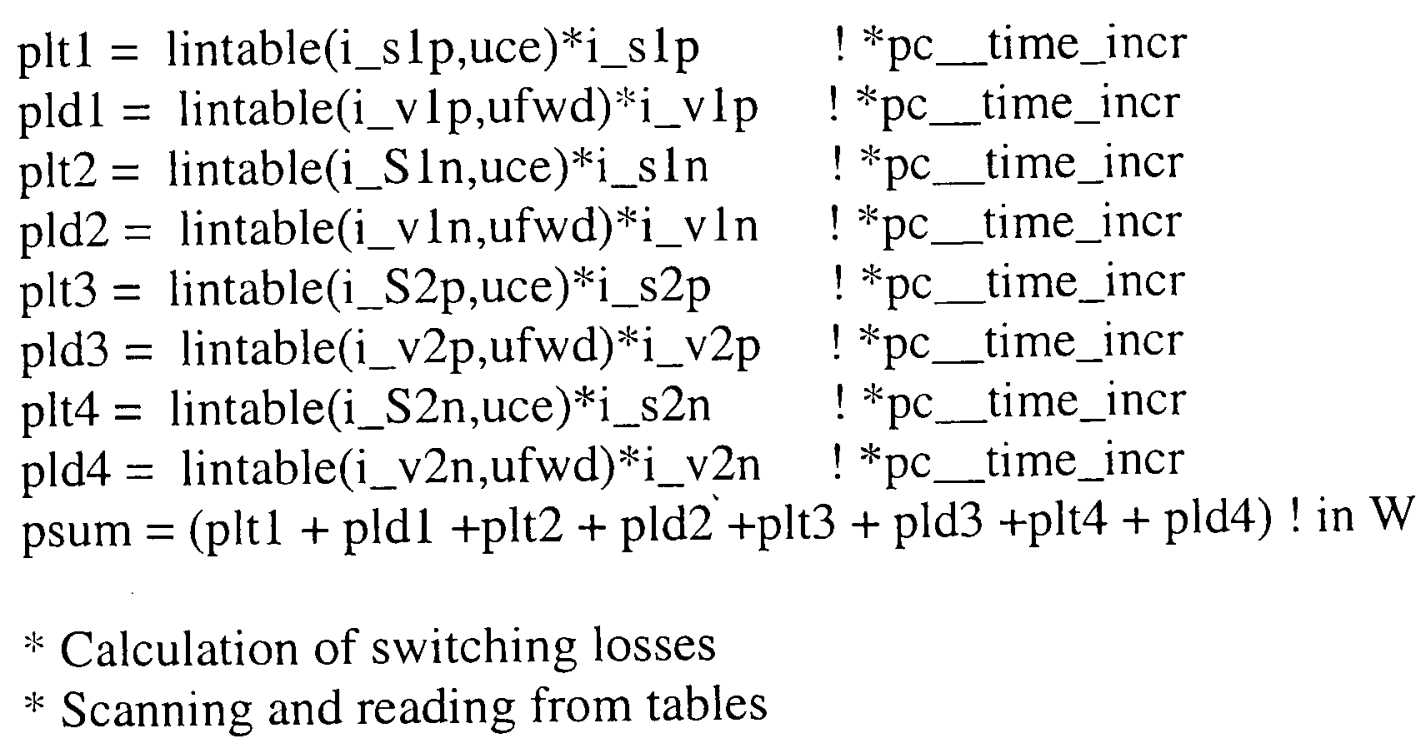

* Calculation of switching losses

* Scanning and reading from tables

if (i_slp .ne. 0.0001) then

ps $1=$ lintable (pst,i_slp)

else

ps $1=0$.

endif

if (i_sln .ne. 0.0001) then

ps2 $=$ lintable(pschalt,i_sln)

else

ps $2=0$.

endif 


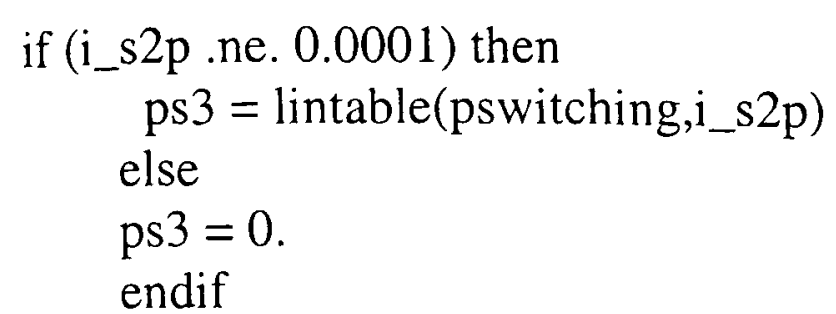

if (i_s $2 n$.ne. 0.0001) then

ps4 = lintable(ps,i_s2n)

else

ps $4=0$.

endif

if (time .gt. 60.e-3) finish

endsection

runcontrols

file: 'run.txt'

endsection

outresults

file: 'out.txt'

endsection

Endend 
Appendix D

Circuit Implementation 
Table D.1: Printed Wiring Board

\begin{tabular}{|l|l|l|l|}
\hline * GND & A 1 & C1 & * GND \\
\hline Temp & A 2 & C2 & Temp \\
\hline V sin L1 & A 3 & C3 & I Comp L1 \\
\hline V sin L2 & A 4 & C4 & I Comp L2 \\
\hline V sin L3 & A 5 & C5 & I Comp L3 \\
\hline +V ZW & A 6 & C6 & - V ZW \\
\hline ERR V & A 7 & C7 & AC-V \\
\hline + HB L3 & A 8 & C8 & - HB L3 \\
\hline + HB L2 & A 9 & C9 & - HB L2 \\
\hline + HB L1 & A10 & C10 & - HB L1 \\
\hline (1) +15V & A11 & C11 & $(1)+15 V$ \\
\hline Vent/24V & A12 & C12 & $(2)-15 V$ \\
\hline GND & A13 & C13 & GND \\
\hline Comp I & A14 & C14 & Comp III \\
\hline Comp II & A15 & C15 & Comp IV \\
\hline Current TI I & A16 & C16 & Current TIII \\
\hline Current TII & A17 & C17 & Current TIV \\
\hline $\begin{array}{l}\text { Clock } \\
\text { Switch }\end{array}$ & A18 & C18 & Phase control \\
\hline (Start) & A19 & C19 & 10/20\% I \\
\hline & A20 & C20 & \\
\hline & A21 & C21 & \\
\hline & A22 & C22 & V \\
\hline *GND & A23 & C23 & * GND \\
\hline Synch L3 & A24 & C24 & I L3 \\
\hline Synch L2 & A25 & C25 & I L2 \\
\hline Synch L1 & A26 & C26 & I L1 \\
\hline (1) +15V & A27 & C27 & $(1)+15 V$ \\
\hline (2)- 15V & A28 & C28 & $(2)-15 V$ \\
\hline$+5 V$ & A29 & C29 & +5V \\
\hline $\begin{array}{l}\text { Auxiliary } \\
\text { Relay }\end{array}$ & A30 & C30 & Main relay \\
\hline & A31 & C31 & $(3)+25 V$ \\
\hline GND & A32 & C32 & $(3)+25 V$ \\
\hline
\end{tabular}




\begin{tabular}{|l|l|l|l|l|l|l|}
\hline Synch & L1 & L2 & L3 & NT & Control & Process \\
\hline
\end{tabular}

\begin{tabular}{|l|l|l|}
\hline$(1)+15 \mathrm{~V}$ & $(3)+25 \mathrm{~V}$ & $(2)-15 \mathrm{~V}$ \\
\hline
\end{tabular}

Table D.2: Driver board

\begin{tabular}{|l|c|c|l|}
\hline GND & 1 & 2 & GND \\
\hline Temp & 3 & 4 & Temp \\
& 5 & 6 & I Comp L1 \\
\hline & 7 & 8 & I Comp L2 \\
\hline & 9 & 10 & I Comp L3 \\
\hline + V ZW & 11 & 12 & - V $_{\text {ZW }}$ \\
\hline ERR V & 13 & 14 & V DS \\
\hline + HB L3 & 15 & 16 & - HB L3 \\
\hline + HB L2 & 17 & 18 & - HB L2 \\
\hline+ HB L1 & 19 & 20 & - HB L1 \\
\hline$+15 \mathrm{~V}$ & 21 & 22 & $+15 \mathrm{~V}$ \\
\hline Vent/24VDC & 23 & 24 & $-15 \mathrm{~V}$ \\
\hline GND & 25 & 26 & GND \\
\hline
\end{tabular}

Table D.3: Network board:

\begin{tabular}{|l|c|c|l|}
\hline GND & 1 & 2 & GND \\
\hline V synch L3 & 3 & 4 & I Net L3 \\
\hline V synch L2 & 5 & 6 & I Net L2 \\
\hline V synch L1 & 7 & 8 & I Net L3 \\
\hline$+15 \mathrm{~V}$ & 9 & 10 & $+15 \mathrm{~V}$ \\
\hline$-15 \mathrm{~V}$ & 11 & 12 & $-15 \mathrm{~V}$ \\
\hline GND & 13 & 14 & GND \\
\hline $\begin{array}{l}\text { Auxiliary } \\
\text { relay }\end{array}$ & 15 & 16 & Main relay \\
\hline$+25 \mathrm{~V}$ & 17 & 18 & $+25 \mathrm{~V}$ \\
\hline GND & 19 & 20 & GND \\
\hline
\end{tabular}


Table D.4: Network ratio

\begin{tabular}{|l|l|l|l|}
\hline I Network & Ratio & V/I Net $/ \mathrm{V}=5$ & \\
\hline $200 \mathrm{~A} / 5 \mathrm{~A}$ & 2.857 & $2,45 \mathrm{~V} /$ & 0 \\
\hline $200 \mathrm{~A} / 1 \mathrm{~A}$ & $2.857 / 5$ & $2,45 \mathrm{~V} / 12,25 \mathrm{~V}$ & 1 \\
\hline $300 \mathrm{~A} / 5 \mathrm{~A}$ & 4.286 & & 2 \\
\hline $300 \mathrm{~A} / 1 \mathrm{~A}$ & $4.286 / 5$ & & 3 \\
\hline $500 \mathrm{~A} / 5 \mathrm{~A}$ & 7.143 & & 4 \\
\hline $500 \mathrm{~A} / 1 \mathrm{~A}$ & $7.143 / 5$ & & 5 \\
\hline $800 \mathrm{~A} / 5 \mathrm{~A}$ & 11.429 & & 6 \\
\hline $800 \mathrm{~A} / 1 \mathrm{~A}$ & $11.429 / 5$ & & 7 \\
\hline $1000 \mathrm{~A} / 5 \mathrm{~A}$ & 14.286 & $0,49 \mathrm{~V}$ & 8 \\
\hline $1000 \mathrm{~A} / 1 \mathrm{~A}$ & $14.286 / 5$ & & 9 \\
\hline
\end{tabular}

Compensation current:70A $I / 100 \mathrm{~A}, i_{\max } / 3,5 \mathrm{~V} / 5 \mathrm{~V}$ Current transformer, amplified to $7 \mathrm{~V} / 10 \mathrm{~V}$

Output 5A, 5A/7A,

Voltage: $230 \mathrm{~V} / 2,3 \mathrm{~V} / 700 \mathrm{~V} / 7 \mathrm{~V} \quad+/-3,5 \mathrm{~V}$

$20 \mathrm{~V} \min / 460 \mathrm{~V} \max 440 \mathrm{~V}, 650 \mathrm{VDC}$

Table D.5: Compensation adjustment

\begin{tabular}{|l|l|l|l|l|l|l|l|l|l|}
\hline 1 & 2 & 3 & 4 & 5 & 6 & 7 & 8 & 9 & 0 \\
\hline $10 \%$ & $20 \%$ & $30 \%$ & $40 \%$ & $50 \%$ & $60 \%$ & $70 \%$ & $80 \%$ & $90 \%$ & $100 \%$ \\
\hline
\end{tabular}

Table D.6: $\cos \varphi$ adjustment

\begin{tabular}{|l|l|l|l|l|l|l|l|l|l|}
\hline 1 & 2 & 3 & 4 & 5 & 6 & 7 & 8 & 9 & 0 \\
\hline 0,8 cap & 0,8 cap & 0,9 cap & 0,9 cap & 1 & 1 & 0,9 ind & 0,9 ind & 0,8 ind & 0,8 ind \\
\hline $10 \%$ & $20 \%$ & $10 \%$ & $20 \%$ & $10 \%$ & $20 \%$ & $10 \%$ & $20 \%$ & $10 \%$ & $20 \%$ \\
\hline
\end{tabular}

Table D.7: Digital switch

\begin{tabular}{|l|l|l|l|l|l|l|l|l|l|l|}
\hline & 1 & 2 & 3 & 4 & 5 & 6 & 7 & 8 & 9 & 0 \\
\hline S0 & $\mathrm{X}$ & & $\mathrm{X}$ & & $\mathrm{X}$ & & $\mathrm{X}$ & & $\mathrm{X}$ & \\
\hline S1 & & $\mathrm{X}$ & $\mathrm{X}$ & & & $\mathrm{X}$ & $\mathrm{X}$ & & & \\
\hline S2 & & & & $\mathrm{X}$ & $\mathrm{X}$ & $\mathrm{X}$ & $\mathrm{X}$ & & $\mathrm{X}$ \\
\hline S3 & & & & & & & & $\mathrm{X}$ & \\
\hline
\end{tabular}


Appendix E

Power and Control Circuit 


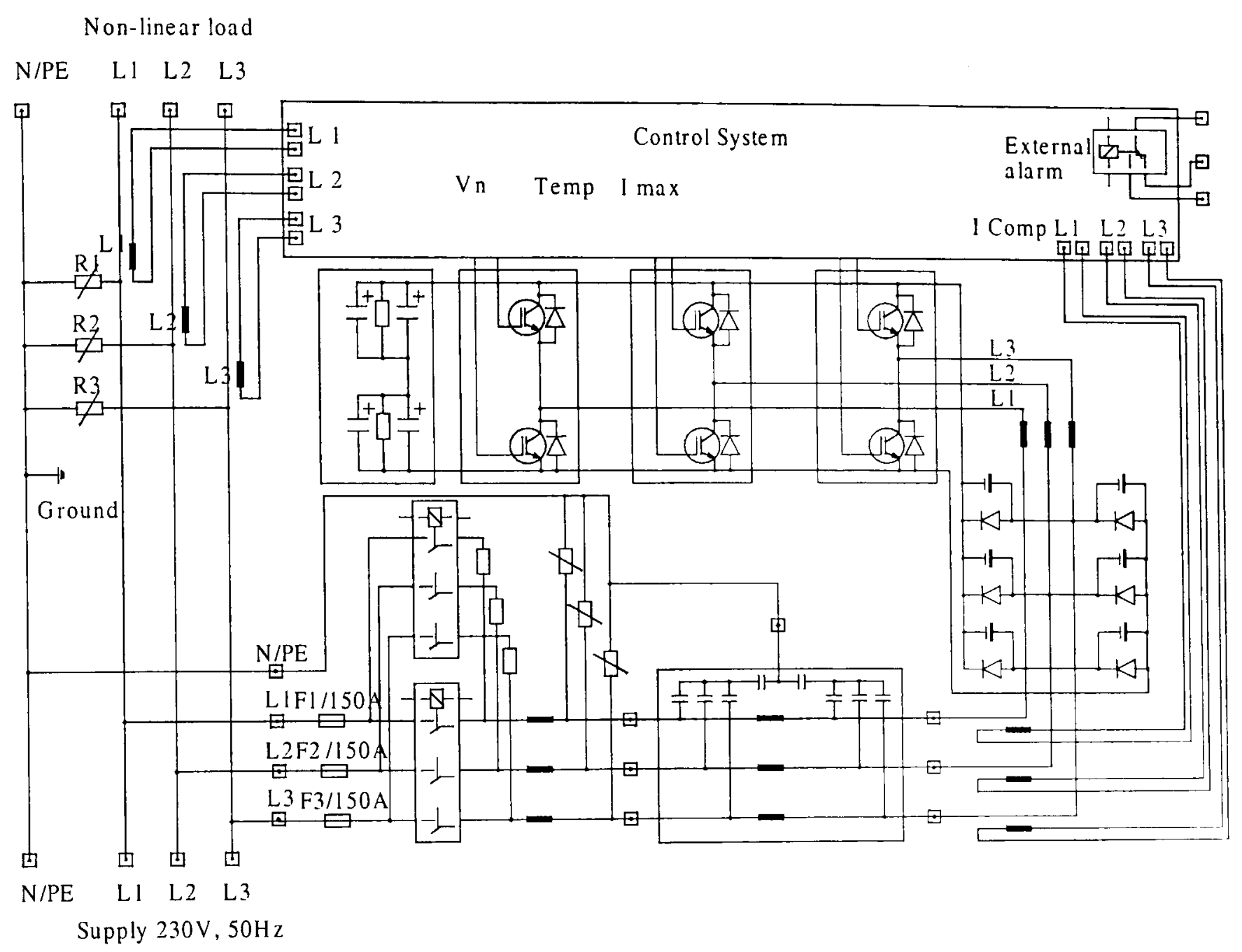

Fig. E1: General Block Diagram of the proposed System 


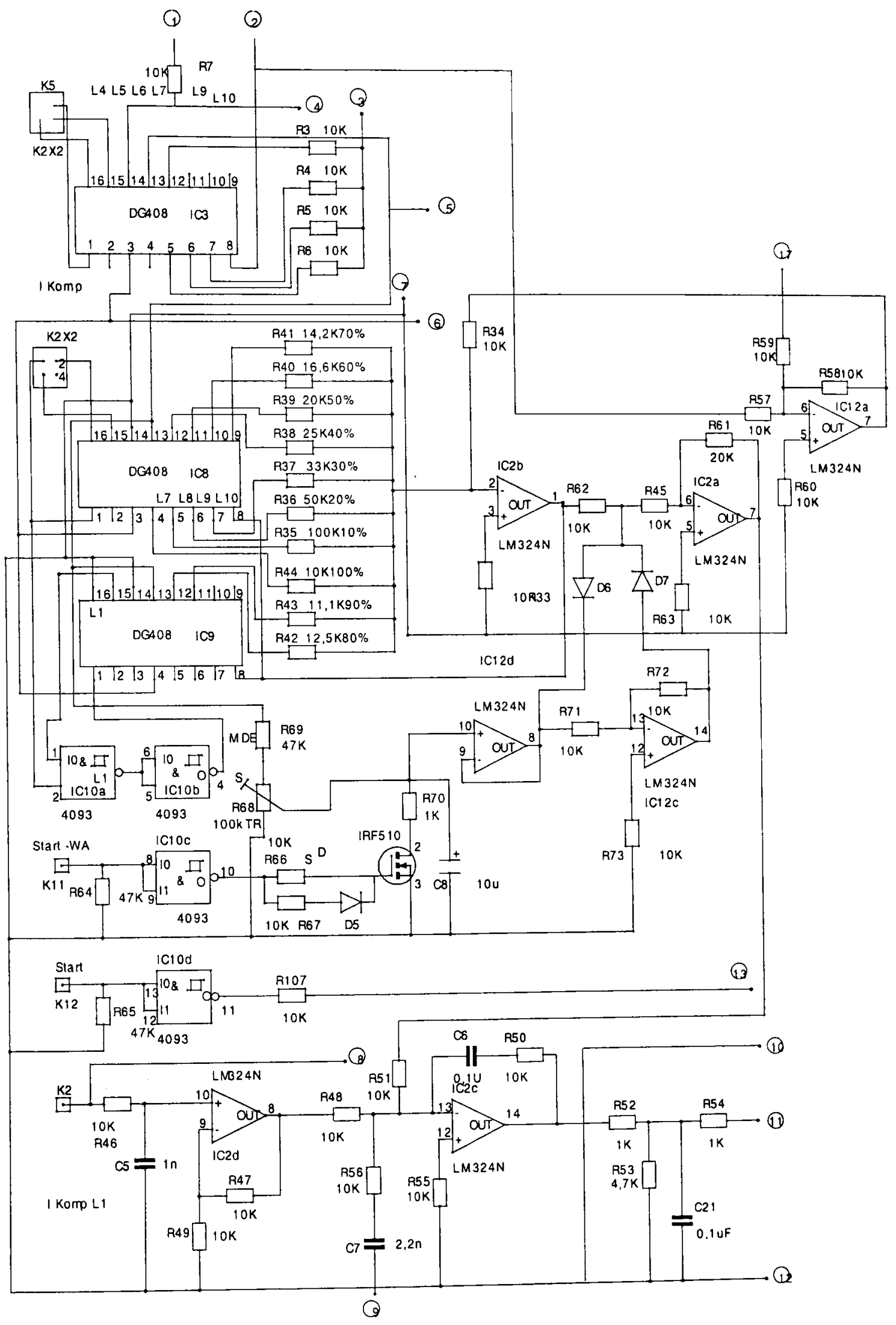

Fig. E2: Diagram of the $\cos \varphi$-Adjustment and its Control System 

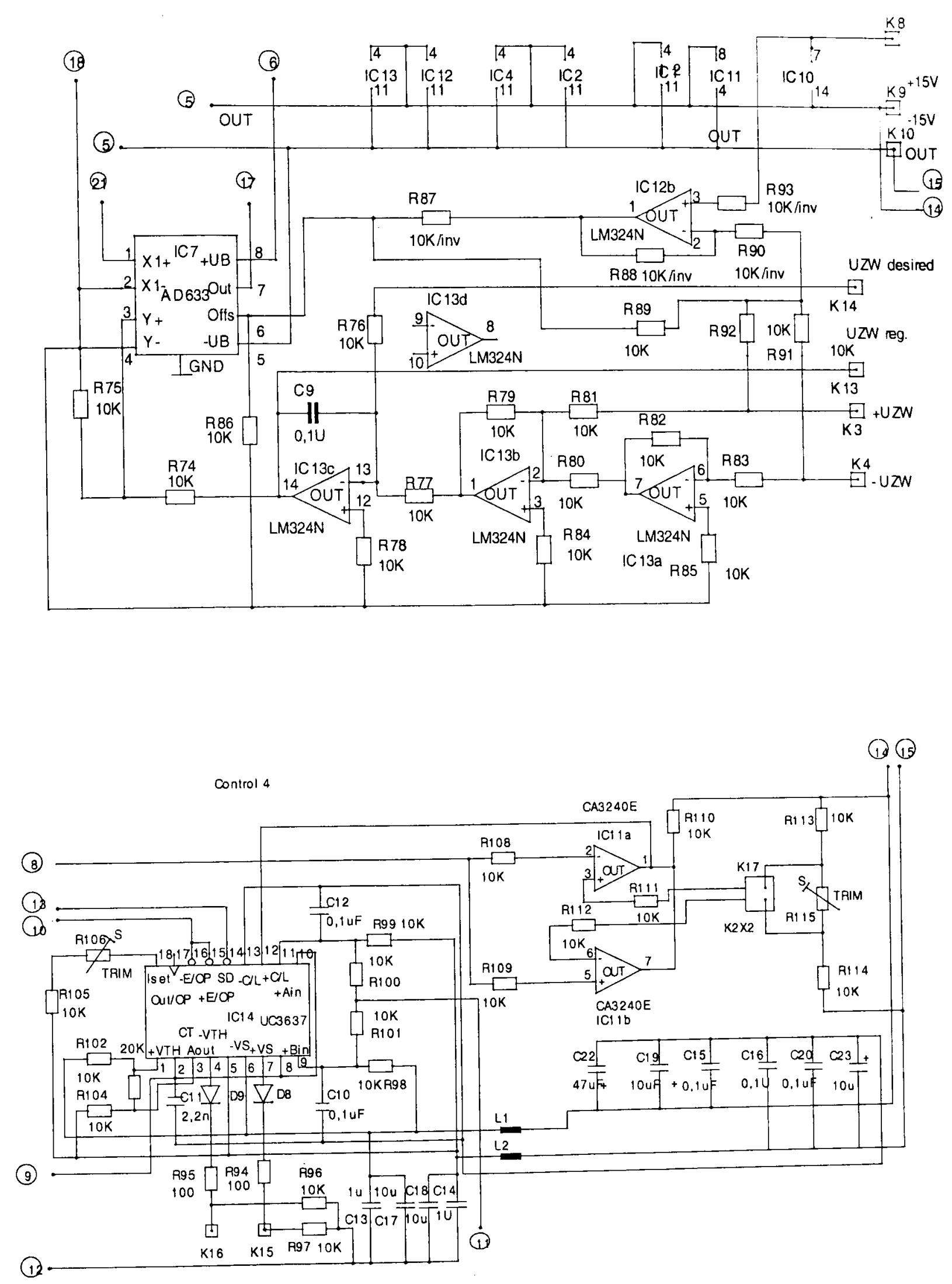

Fig. E3: Block Diagram of the Hystersis and PWM-Control 

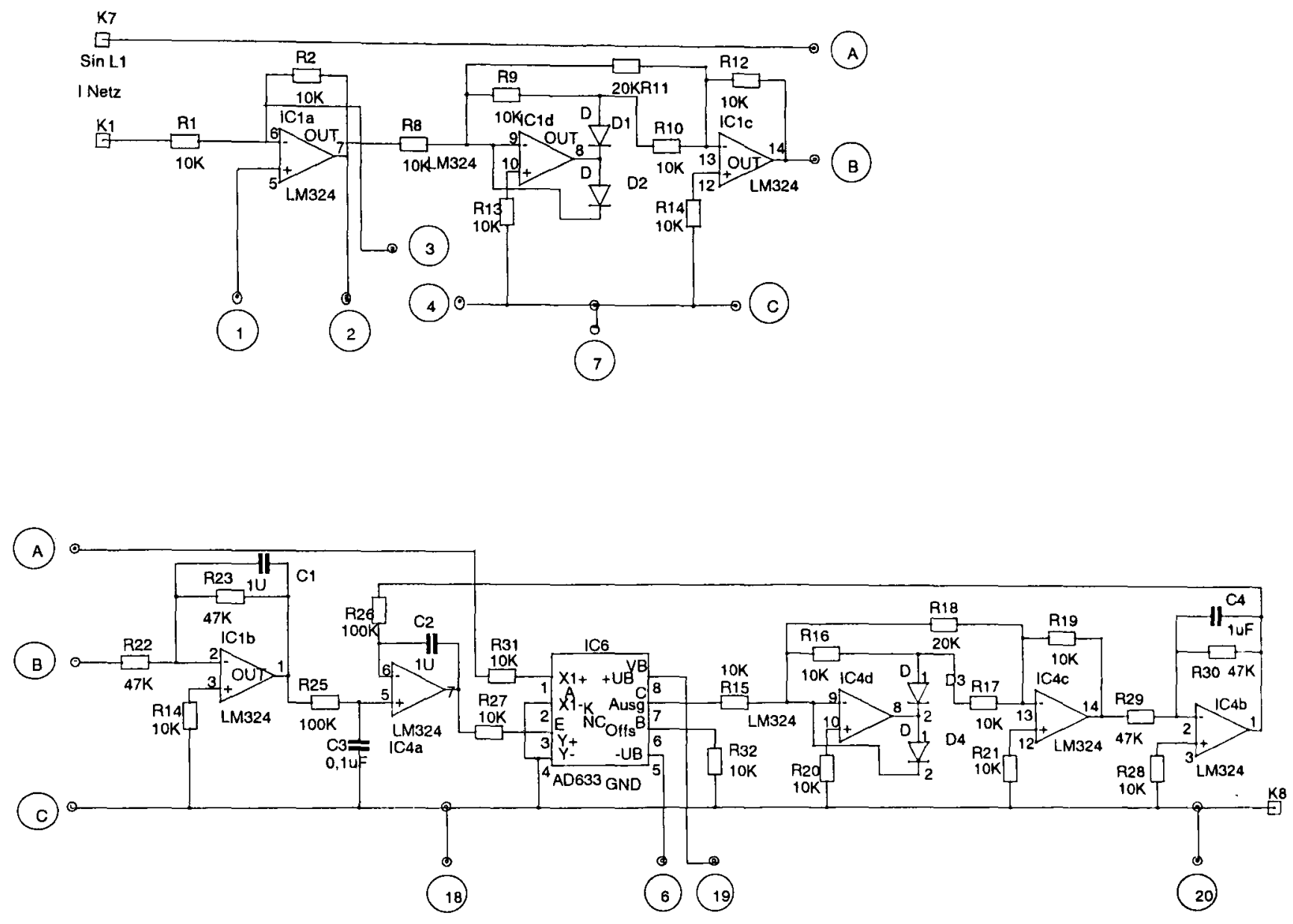

Fig. E4: Comparision of the Main Supply and Reference current 
Appendix F

Overview and Construction of the Proposed System 
Overview and Construction of the Proposed System
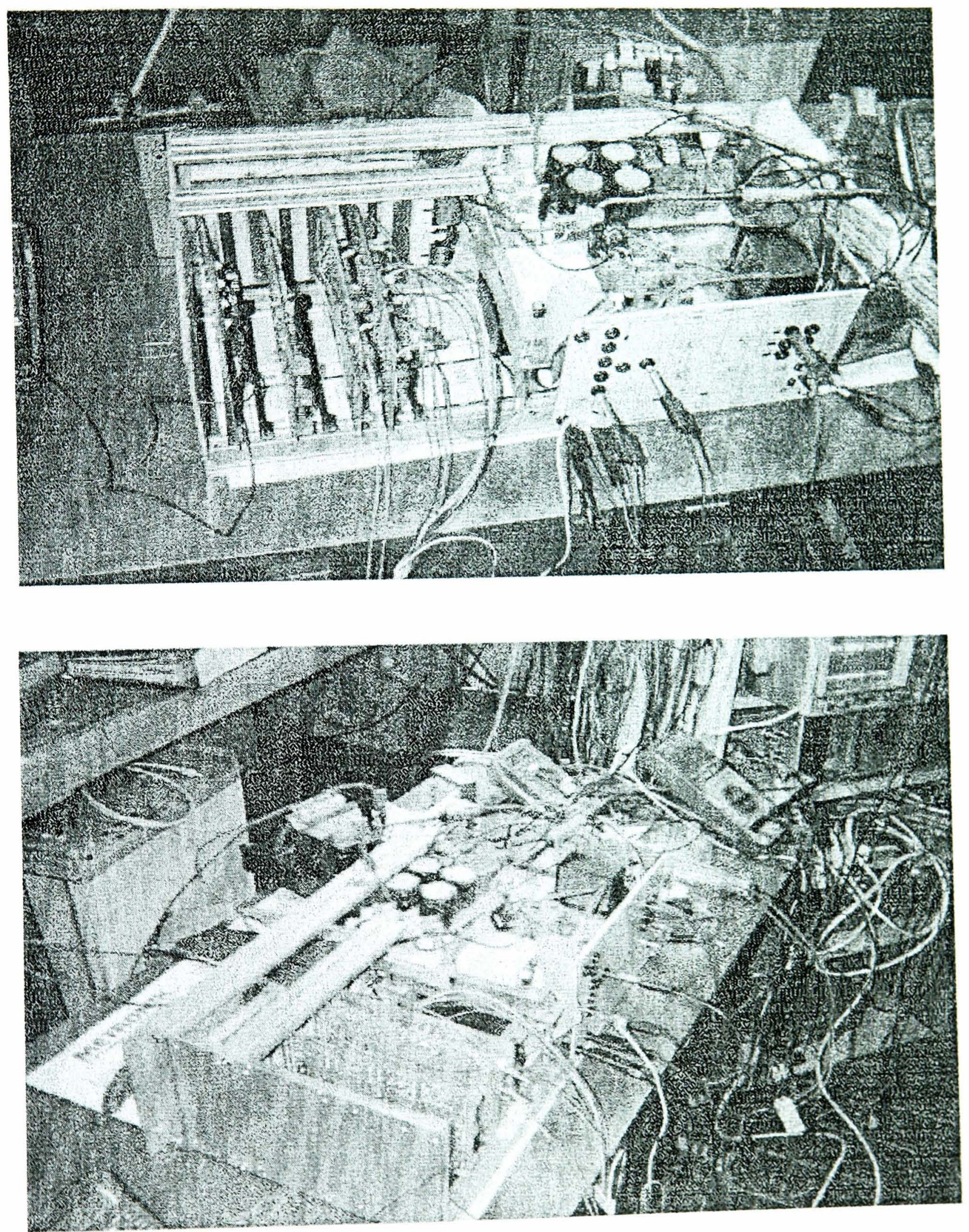
Appendix G

List of Publications 


\section{List of Publications (papers)}

1. Kasikci I., Darwish M.K., Mehta P., "A New Method for Harmonic Reduction"' LESCOPE'99, June 1999, pp 170-174, Halifax, Canada.

2. Kasikci I., Darwish M.K., Mehta P., "Load Modelling for the Reactive Power Compensation', LESCOPE'99, June 1999, pp 185-188, Halifax, Canada.

3. Kasikci I., Darwish M.K., Mehta P., "A New Method for Harmonic/Reactive Power Reduction', IEEE PEDS'99 International Conference, 0-7803-5769-8/99 \$10.00 1999 IEEE, pp 900-904, July 1999, Hong Kong.

4. Kasikci I., Darwish M.K., Mehta P., "Analytical Load Model for Power System Components', IEEE PEDS'99 International Conference,, 0-7803-5769-8/99 \$10.00 1999 IEEE, pp 673-676, July 1999, Hong Kong.

5. Kasikci I., “A New Method for Power Quality Improvement', IEEE Power Engineering Society, International Conference, page 297 (summary), Power Tech'99, Budapest, Hungary.

6. Kasikci I., Darwish M.K., Mehta P., "A New method for harmonic reduction in low and medium voltage distribution systems', UPEC'99, Leicester, UK.

7. Kasikci I., Darwish M.K., Mehta P., "Load modelling for reactive/harmonic power compensation', UPEC'99, Leicester, UK.

8. Kasikci I., Darwish M.K., Mehta P., "A New Contribution into the Improvement of Power Factor Correction", IEEE, IEE Conference, DRPT 2000. City University, UK. 
9. Kasikci I., "Reactive Power Compensation in Low and Medium Power Systems", $6^{\text {th }}$ Power, Computer and Electronic National Conference, 1996, Bursa, Turkey.

10. Kasikci I., "Harmonics and Grounding Problems in Low and Medium Power Systems", $7^{\text {th }}$ Power, Computer and Electronic National Conference, 1998, Ankara, Meadle East University of Turkey.

11. Kasikci I., Darwish M.K., Mehta P., "Modelling and Analysis of Power Systems Loads and Harmonic Flow Calculations ", IEEE-PES Turkey Chapter, ELECO'99 International Conference on Electrical and Eletronics Engineering, Bursa / Turkey.

12. Kasikci I., Darwish M.K., Mehta P., "Power Quality Problems and its Solutions', IEEE-PES Turkey Chapter, ELECO'99 International Conference on Electrical and Eletronics Engineering, Bursa / Turkey. 